University of Louisville ThinkIR: The University of Louisville's Institutional Repository

Electronic Theses and Dissertations

$5-2013$

\title{
In-situ monitoring of transport properties of graphene during plasma functionalization and it's applications in energy storage.
}

Ruwantha Jayasingha Atapattu Mudiyaneselage 1980-

University of Louisville

Follow this and additional works at: https://ir.library.louisville.edu/etd

\section{Recommended Citation}

Atapattu Mudiyaneselage, Ruwantha Jayasingha 1980-, "In-situ monitoring of transport properties of graphene during plasma functionalization and it's applications in energy storage." (2013). Electronic Theses and Dissertations. Paper 55.

https://doi.org/10.18297/etd/55

This Doctoral Dissertation is brought to you for free and open access by ThinkIR: The University of Louisville's Institutional Repository. It has been accepted for inclusion in Electronic Theses and Dissertations by an authorized administrator of ThinkIR: The University of Louisville's Institutional Repository. This title appears here courtesy of the author, who has retained all other copyrights. For more information, please contact thinkir@louisville.edu. 


\title{
IN-SITU MONITORING OF TRANSPORT PROPERTIES OF GRAPHENE DURING PLASMA FUNCTIONALIZATION AND IT'S APPLICATIONS IN ENERGY STORAGE
}

\author{
By \\ Ruwantha Jayasingha, Atapattu Mudiyaneselage \\ BSc., University of Kelaniya, Sri Lanka \\ M.S., University of Louisville
}

\begin{abstract}
A Dissertation
Submitted to the Faculty of the

College of Arts and Sciences of the University of Louisville

In Partial Fulfillment of the Requirements

For the Degree of
\end{abstract}

Doctor of Philosophy

Department of Physics

University of Louisville

Louisville, Kentucky

May 2013 



\title{
IN-SITU MONITORING OF TRANSPORT PROPERTIES OF GRAPHENE DURING PLASMA FUNCTIONALIZATION AND IT'S APPLICATIONS IN ENERGY STORAGE
}

\author{
By \\ Ruwantha Jayasingha, Atapattu Mudiyaneselage \\ BSc. University of Kelaniya, Sri Lanka \\ M.S., University of Louisville \\ A Thesis Approved on
}

April 26, 2013

By the Following Thesis Committee:

Dr. Gamini Sumanasekara (Thesis Director)

Dr. C.S. Jayanthi

Dr. Shi-Yu Wu

Dr. Chris L. Davis

Dr Shamus P. McNamara 


\section{ACKNOWLEDGEMENTS}

I am heartily thankful to my thesis advisor Dr. Gamini Sumanasekara, whose encouragement, guidance and support from the initial to the final level enabled me to develop an understanding of the subject. I attribute the level of my $\mathrm{PhD}$ degree to his encouragement and effort and without him this thesis, too, would not have been completed or written. One simply could not wish for a better or friendlier supervisor. I would like to thank my committee members Dr. C.S. Jayanthi, Dr. Shi-Yu Wu, Dr. Chris L. Davis and Dr Shamus P. McNamara for the guidance and being in my thesis committee.

I would also like to thank my lab colleagues for their support. Specially, I would like to express my gratitude to Dr. Arjun thapa, Dr. Jacek Jasinski and Jeong Kim for sharing all their knowledge. Also many thanks go to Andriy Sherehiy, Kasun Fernando and Robert Stallard for making the lab experience educational and enjoyable.

I would like to express the deepest appreciation to my parents for their endless love and encouragement throughout my life. Finally I would like to thank my wife Isuru, for her great patience, constant encouragement and endless love for making this thesis a success.

Lastly, I offer my regards to all of those who supported me in any respect during the completion of my thesis. 


\title{
ABSTRACT \\ IN-SITU MONITORING OF TRANSPORT PROPERTIES OF GRAPHENE DURING PLASMA FUNCTIONALIZATION AND IT'S APPLICATIONS IN ENERGY STORAGE
}

\author{
Ruwantha Jayasingha, Atapattu Mudiyaneselage \\ $26^{\text {th }}$ April 2013
}

Monolayer Graphene synthesized by chemical vapor deposition was subjected to controlled and sequential hydrogenation using RF plasma while monitoring its electrical properties in-situ. Low temperature transport properties were measured for each sample and correlated with ex-situ Raman scattering and X-ray photoemission characteristics. The dominant transport mechanism for weak hydrogenation was found to be electron diffusion and low temperature transport for strong hydrogenation is governed by variable range hopping. This investigation of transport properties of hydrogenated graphene supports to develop the universal scaling functions for metallic and insulating graphene by identifying the critical hydrogen concentration. A clear transition from Weak to Strong localization was identified by the pronounced negative magneto resistance. Variable temperature measurements done on sequentially fluorinated graphene too demonstrated a transition from metallic to semiconductor behavior. The temperature dependence of resistance supports the emergence of a bandgap in the fluorinated graphene films. Controllably fluorinated carbon nanotubes showed promise for high capacity primary and secondary battery performances. 


\section{TABLE OF CONTENTS}

PAGE

ACKNOWLEDGEMENTS ..................................................

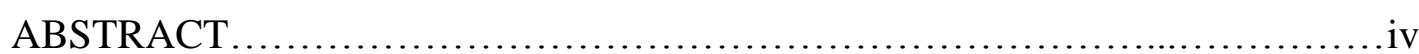

LIST OF FIGURES ...................................................... viii

CHAPTER 01 - INTRODUCTION.........................................

1.1 Carbon Atom and its Hybridizations........................ 01

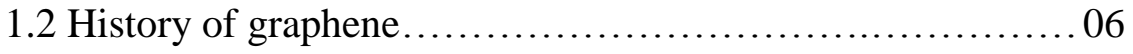

1.3 Band structure of Graphene............................... 08

1.4 Synthesis of graphene................................ 16

1.4.1 Mechanical exfoliation........................... 17

1.4.2 Epitaxial graphene on silicon carbides............... 18

1.4.3 Wet Chemical synthesis of graphene................ 19

1.5 Band gap engineering of graphene...................... 20

1.5.1 Substrate induced band gap opening................. 21

1.5.2 Chemical substitution doping....................... 22

1.5.3 Hybrids........................................... 24

1.5.4 Graphene nanoribbons............................. 25

1.6 Scaling theory of localization...........................27

1.6.1 Localization within scaling theory................... 28

1.6.2 Scaling theory of $\beta$ function......................... 29 
CHAPTER 02 - CHEMICAL VAPOR DEPOSITION OF GRAPHENE: SYNTHESIS AND CHARACTERIZATION

2.1 Introduction 34

2.2 Kinetics and mass transport mechanisms of CVD synthesis.. 35

2.3 Synthesis of Graphene by chemical vapor deposition .42

2.4 Chemical Vapor Deposition of Graphene: Characterization.. 45

2.4.1 Scanning electron microscope .45

2.4.2 Raman Spectroscopy............................ 50

2.5 Graphene transfer process............................ 56

\section{CHAPTER 03 - HYDROGEN FUNCTIONALIZATION OF GRAPHENE}

3.1 Introduction ......................................... 60

3.2 Transport measurements on graphene.................... 62

3.2.1 Thermoelectric power of Graphene...................63

3.2.2 Four Probe Resistivity - the van der Pauw Method.....67

3.2.3 Hall mobility measurements.......................69

$3.3 \mathrm{in}$-situ functionalization of graphene......................71

3.4 ex-situ functionalization of graphene..................... 77

3.4.1 Low temperature transport measurements............. 77

3.4.2 Raman and XPS characterization of graphene......... 90

CHAPTER 04 - FLUORINE FUNCTIONALIZATION OF GRAPHENE: EVIDENCE OF A BAND GAP

4.1 Introduction ....................................... 95

4.2 Fluorination of Graphene.............................. 96 
4.3 In-situ study of controlled fluorination of graphene. 97

4.4 ex-situ functionalization of graphene. 100

4.4.1 Low temperature transport measurements. 100

\section{CHAPTER 05 - OPTIMIZATION OF MWNT BASED CFX ELECTRODES FOR PRIMARY AND SECONDARY BATTERIES}

5.1 Introduction 114

5.2 Fluorine functionalization of MWCNT 117

5.3 Primary battery (Li/CFx ) performance. .123

5.4 Secondary battery (Li/CFx ) performance. 124

CHAPTER 06 - CONCLUSIONS. 130

REFERENCES..... 132

CURRICULUM VITAE. 144 


\section{LIST OF FIGURES}

FIGURE

PAGE

1.1 Electronic configurations for carbon in the ground state and in the excited state

1.2 Formation of sp hybrid orbitals and the schematic presentation of acetylene

1.3 Formation of $\mathrm{sp}^{2}$ hybrid orbitals and the benzene ring

$1.4 \quad$ Formation of $\mathrm{sp}^{3}$ hybrid orbitals 05

1.5 Graphene as the 2D building material for carbon materials of all other 07 dimensionalities

1.6 Graphene hexagonal lattice structure and the corresponding Brillouin 09 zone

1.7 Electronic dispersion in the honeycomb lattice

$\begin{array}{lll}1.8 & \text { Scalable techniques of graphene production } & 17\end{array}$

1.9 Atomic force map of $6 \mathrm{H}-\mathrm{SiC}$ after graphene growth. 20

1.10 Substrate induced band gap opening in graphene on SiC substrate 21

1.11 Band gap opening by substitutional doping of nitrogen in graphene 22 lattice

1.12 Making GNRs from CNTs

1.13 Typical wave function for delocalized and localized states, 30

1.14 Schematic-function for the electrical metal-insulator transition in 31 different dimensions, in the presence of time-reversal symmetry

$2.1 \quad$ Sequence of events during CVD 36

2.2 Boundary layer and velocity changes in a tube reactor 37

2.3 Temperature boundary layer and temperature changes in a tubular $\quad 38$ reactor 
2.5 Illustration of carbon segregation at metal surface 41

2.6 Schematic diagram of the CVD system for graphene synthesis 43

2.7 CVD system for graphene synthesis $\quad 44$

2.8 Graphene growth process $\quad 44$

2.9 Scanning electron microscope column 46

2.10 Electron beam-specimen interaction 48

2.11 Raman spectrum of single layer graphene on $\mathrm{SiO}_{2}$

2.12 Raman 2D band $\quad 54$

2.13 SEM image of graphene on a copper foil with a growth time of $18 \quad 55$ $\min$

2.14 Raman spectra obtained from a graphene film grown on a thin copper 56 film

2.15 Schematic diagram of the etching and scooping method without using 57 a support

2.16 Graphene transfer with PMMA as a support 58

2.17 SEM image of graphene transferred on to $\mathrm{SiO}_{2} / \mathrm{Si}$ substrate 59

3.1 Hydrogenation of Graphene $\quad 61$

3.2 Electrical concretions on the graphene sample 63

3.3 Built in potential due to the temperature gradient in metal 63

3.4 Calculated graphene thermopower for different scattering exponents 66

3.5 Schematic of a Van der Pauw configuration $\quad 68$

3.6 Schematic of measuring Hall voltage $\quad 70$

3.7 Low temperature transport measurement setup 72

3.8 Schematic of the plasma functionalization setup 73

3.9 Plasma functionalization (a) setup (b) in-situ functionalization of $\quad 74$ graphene with $\mathrm{H}_{2}$ plasma (c) Graphene sample mounted on the probe 
3.10 In-situ time evolution of the Resistance, $R(t)$ during hydrogenation.

The arrow heads represent the initiation of intermittent plasma

3.11 Temperature dependence of resistance, $R(T)$ of progressively hydrogenated Graphene samples.

3.12 Temperature dependence of conductance

3.13 Low temperature behavior of the conductivity, $G$ vs $T^{1 / 3}$ plot

3.14 Scaling Plot of the conductivity data

3.15 Variable range hopping analysis for $R(T)$

3.16 $T_{0}$ values for each hydrogenation process characterized by change in room temperature resistance

3.17 Magneto resistance data for progressively hydrogenated Graphene

3.18 Magneto resistance $(\Delta R / R)$ data for progressively hydrogenated Graphene with the best fit for $W L$ theory

3.19 Temperature dependence of Thermoelectric power, $S(T)$

3.20 Temperature dependence of Hall voltage over excitation current, $V_{H} / I \quad 88$ for progressively hydrogenated Graphene

3.21 Temperature dependence of Hall Mobility $(|\mu|(T))$ for progressively hydrogenated Graphene.

3.22 Raman spectra of progressively hydrogenated Graphene

3.23 Ratio of intensities of D and G bands for each hydrogenation

3.24 XPS results for C1S peak of progressively hydrogenated Graphene

4.1 Structure of fluorinated graphene 96

4.2 Schematic of the plasma functionalization setup 98

4.3 In-situ time evolution of the Resistance $R(t)$ and Thermoelectric power $S(t)$ during hydrogenation

4.4 Temperature dependence of resistance, $R(T)$ of progressively fluorinated Graphene samples

4.5 Temperature dependence of conductance

4.6 Low temperature behavior of the conductivity, $G$ vs $T^{1 / 3}$ plot 
4.7 At low temperature, the sample resistance is fit to the twodimensional variable-range hopping model

$\begin{array}{lll}4.8 & T_{0} \text { values for each fluorination processes } & 104\end{array}$

4.9 Magneto resistance data for progressively fluorinated graphene 105

4.10 Temperature dependence of Thermoelectric power 106

4.11 Hall voltage over excitation current, $V_{H} / I$ for progressively 107 fluorinated Graphene

4.12 Temperature dependence of Hall Mobility $(|\mu|(T))$ for progressively 108 fluorinated Graphene

4.13 Raman spectroscopy results for progressively fluorinated Graphene 109

4.14 Raman spectroscopy results for intensities ratio of D and G bands 110

4.15 XPS survey spectra of progressively fluorinated Graphene 111

4.16 XPS results for C1S peak of progressively fluorinated Graphene 112

4.17 The Arrhenius plot of $\log (R)$ vs $\left(1 / k_{B} T\right)$ for densely fluorinated 113 graphene

5.1 (a) TEM image of MWCNT. (b) Diameter distribution of MWCNT. 117

5.2 Schematic diagram of fluorination chamber 118

$\begin{array}{ll}\text { 5.3 Plasma functionalization setup } & 119\end{array}$

5.4 SEM image of MWNTs subjected to a controlled mild fluorination 119

5.5 Survey XPS spectra of pristine and controlled fluorinated MWNTs 120

5.6 X-ray diffraction patterns of the F-MWNT samples 122

5.7 First discharge capacity curves of CFx Lithium primary battery 123

5.8 Cycling stability of the MWCNT with different fluorine 124 concentration

5.9 Discharge and charge profiles of the pure, $2 \mathrm{hr}, 4 \mathrm{hr}$, and $8 \mathrm{hr}$ MWCNTs obtained after one, ten, twenty and thirty cycles

5.10 Cyclic voltammograms for the $1^{\text {st }}$ cycle of pure and plasma fluorinated MWCNT 
5.11 Cycling performance of the Pure and fluorinated MWCNT at various current densities

5.12 Ex-situ XRD for pristine and fluorinated MWNTs after first cycle discharge 


\section{CHAPTER 01}

\section{INTRODUCTION}

\subsection{Carbon Atom and its Hybridizations}

Carbon, the building element of graphene and graphite is the $6^{\text {th }}$ element of the periodic table. The carbon atom consists of 6 protons, A neutrons, and 6 electrons, where $\mathrm{A}=6$ and 7 yield the stable isotopes ${ }^{12} \mathrm{C}$ and ${ }^{13} \mathrm{C}$, respectively. $\mathrm{A}=8$ yields the radioactive isotope ${ }^{14} \mathrm{C}$. The isotope ${ }^{12} \mathrm{C}$ with a nuclear spin $\mathrm{I}=0$ is the most common isotope found in nature with $99 \%$ of all carbon atoms. Only $1 \%$ are ${ }^{13} \mathrm{C}$ with a nuclear spin of $I=1 / 2$. There are only limited traces of ${ }^{14} \mathrm{C}$ which $\beta$-decays into nitrogen ${ }^{14} \mathrm{~N}$. ${ }^{14} \mathrm{C}$ is an important isotope used for historical dating (radio carbon). Measurement of the ${ }^{14} \mathrm{C}$ concentration of an organic material, mainly wood, allows one to date its biological activity up to a maximum age of roughly 80000 years. In general, carbon is the elementary building block of all organic molecules and, therefore, responsible for life on Earth.

There are 6 electrons in the configuration of $1 s^{2} 2 s^{2} 2 p^{2}$ in the atomic ground state of carbon. Here 2 electrons fill the inner shell 1s, which is closer to the nucleus and irrelevant for chemical reactions. The remaining 4 electrons occupy the outer shell of $2 s$ and $2 p$ orbitals. The energy of $2 p$ orbitals $\left(2 p_{x}, 2 p_{y}\right.$, and $\left.2 p_{z}\right)$ are roughly $4 \mathrm{eV}$. Higher than that of $2 \mathrm{~s}$ orbital. Therefore it is energetically favorable to place 2 electrons in the $2 \mathrm{~s}$ orbital and only 2 of them in the $2 \mathrm{p}$ orbitals (Figure 1.1). 
In the presence of other atoms like $\mathrm{H}, \mathrm{O}$, or other $\mathrm{C}$ atoms, it is favorable to excite one electron from the $2 \mathrm{~s}$ to the third $2 \mathrm{p}$ orbital. This will allow $\mathrm{C}$ atom to form covalent bonds with the other atoms. The gain in energy from the covalent bond should be greater than the $4 \mathrm{eV}$, invested in the electronic excitation.

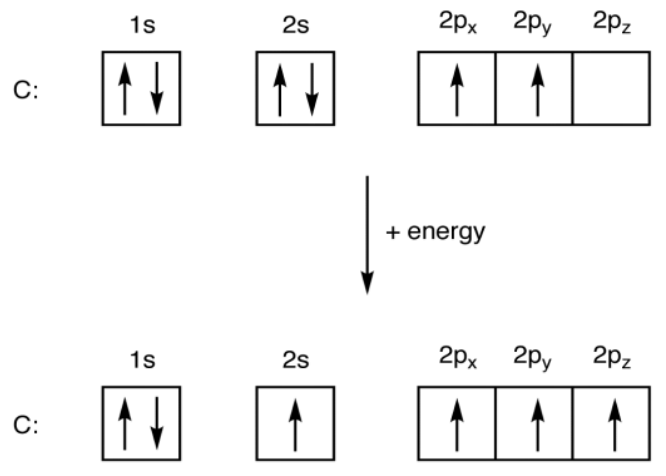

Figure 1.1: Electronic configurations for carbon in the ground state and in the excited state.

In the excited state, we therefore have four equivalent quantum-mechanical states, $|2 s\rangle,\left|2 p_{x}\right\rangle,\left|2 p_{y}\right\rangle$, and $\left|2 p_{z}\right\rangle$. A quantum-mechanical superposition of the state $|2 s\rangle$ with $n \mid 2 p_{j}>$ states is called $s p^{n}$ hybridization, which plays an essential role in covalent carbon bonds[1].

\subsection{1 sp hybridization}

The sp hybridization of carbon atom is formed by mixing of $|2 s\rangle$ state with one of the $2 p$ orbitals. This can be illustrated by choosing the $\left|2 p_{x}\right\rangle$ state. A state with equal weight from both original states, is obtained by the symmetric and anti-symmetric combinations 


$$
\begin{aligned}
\left|s p_{+}\right\rangle & =\frac{1}{2}\left(|2 s\rangle+\left|2 p_{x}\right\rangle\right),\left|s p_{-}\right\rangle \\
& =\frac{1}{2}\left(|2 s\rangle-\left|2 p_{x}\right\rangle\right)
\end{aligned}
$$

The other states, $\left|2 p_{y}\right\rangle$ and $\left|2 p_{z}\right\rangle$, remain unaffected by this superposition. The electronic density of the hybridized orbitals has the form of a club and is elongated in the $+\mathrm{x}(-\mathrm{x})$ direction for the $\left|\mathrm{sp}_{+}\right\rangle\left(\left|\mathrm{sp}_{-}\right\rangle\right)$states.
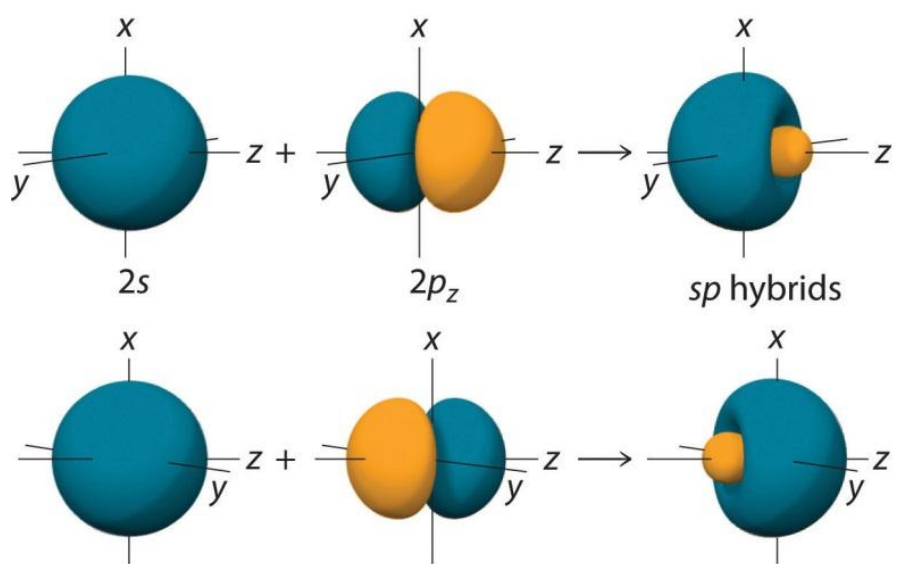

H 1 s

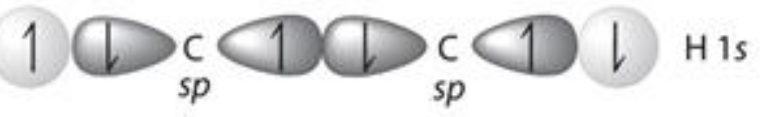

$\mathrm{C}_{2} \mathrm{H}_{2} \sigma$-bonded framework

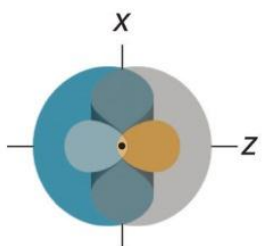

Hybrids shown together in cross-section

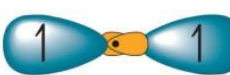

Schematic representation of hybrids shown together

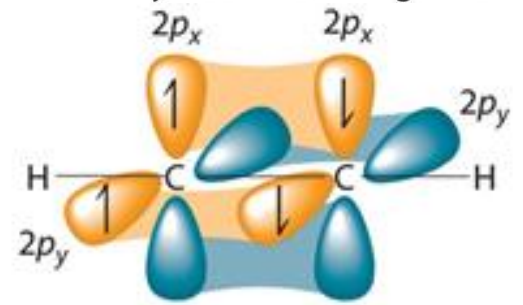

$\mathrm{C}_{2} \mathrm{H}_{2} \pi$ bonding

Figure 1.2: Formation of sp hybrid orbitals and the schematic presentation of acetylene[2]

An example of $\mathrm{sp}$ hybridization as exhibited in the formation of the acetylene molecule $\mathrm{H}-\mathrm{C} \equiv \mathrm{C}-\mathrm{H}$ is shown in Fig. 1.2. The overlapping sp orbitals of the two carbon atoms form a strong covalent $\sigma$ bond. The remaining un-hybridized $2 p$ orbitals are furthermore involved in the formation of two additional $\pi$ bonds. These $\pi$ bonds are weaker than the $\sigma$ bond. 


\subsection{2 $\mathrm{Sp}^{2}$ hybridization}

$\mathrm{Sp}^{2}$ hybridization will result from the superposition of the $2 \mathrm{~s}$ and two $2 \mathrm{p}$ orbitals. The planar $\mathrm{sp}^{2}$ hybridization choose the $\left|2 \mathrm{p}_{\mathrm{x}}\right\rangle$ and the $\left|2 \mathrm{p}_{\mathrm{y}}\right\rangle$ states. So the three quantummechanical states are given by,

$$
\begin{gathered}
\left|s p_{1}^{2}\right\rangle=\frac{1}{\sqrt{3}}|2 s\rangle+\sqrt{\frac{2}{3}}\left|2 p_{y}\right\rangle \\
\left|s p_{2}^{2}\right\rangle=\frac{1}{\sqrt{3}}|2 s\rangle+\sqrt{\frac{2}{3}}\left(\frac{\sqrt{3}}{2}\left|2 p_{x}\right\rangle+\frac{1}{2}\left|2 p_{y}\right\rangle\right) \\
\left|s p_{3}^{2}\right\rangle=-\frac{1}{\sqrt{3}}|2 s\rangle \\
+\sqrt{\frac{2}{3}}\left(-\frac{\sqrt{3}}{2}\left|2 p_{x}\right\rangle+\frac{1}{2}\left|2 p_{y}\right\rangle\right)
\end{gathered}
$$

These orbitals are oriented in the xy-plane and have mutual $120^{\circ}$ angles as shown in Figure 1.3. The remaining un-hybridized $2 \mathrm{p}_{z}$ orbital is perpendicular to the plane.
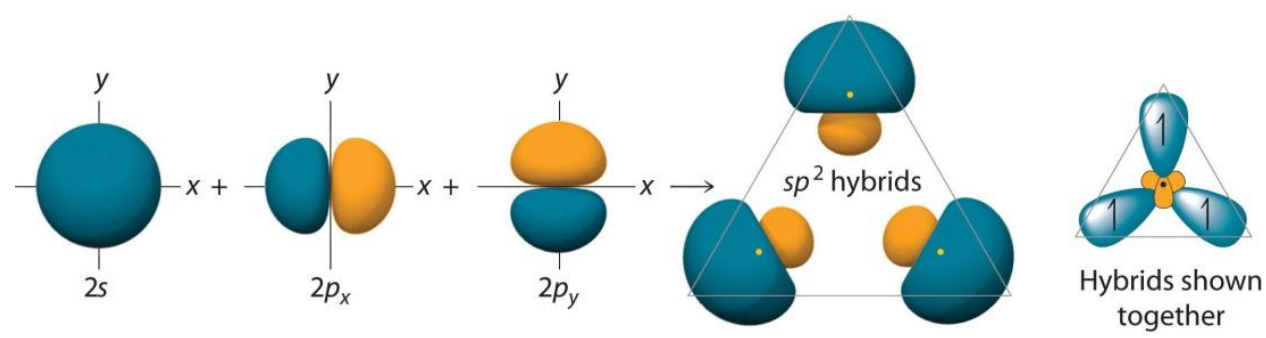<smiles>c1ccccc1</smiles>

Figure 1.3: Formation of $\mathrm{sp}^{2}$ hybrid orbitals and the benzene ring [2]

Benzene molecule is a prominent chemical example for $\mathrm{sp}^{2}$ hybridization. This molecule consists of a hexagon with carbon atoms at the corners linked by $\sigma$ bonds. 
Each carbon atom has covalently bond with one hydrogen atoms which stick out from the hexagon in a star-like manner. In addition to the $6 \sigma$ bonds, the remaining $2 p_{z}$ orbitals form $3 \pi$ bonds, and the resulting double bonds alternate with single $\sigma$ bonds around the hexagon. All measured carbon-carbon distance in benzene is $0.142 \mathrm{~nm}$ for all bonds. This is roughly the average length of both single and double bond.

\subsection{3 $\mathrm{Sp}^{3}$ hybridization}

The $\mathrm{sp}^{3}$ hybridization can be obtained by superposing the $2 \mathrm{~s}$ and all three $2 \mathrm{p}$ orbitals. This consists of four club-like orbitals that make a tetrahedron as in Figure 4. The orbitals are formed at angles of $109.5^{\circ}$ degrees. Methane $\left(\mathrm{CH}_{4}\right)$ is a good chemical example of this hybridization where the four hybridized orbitals are used to form covalent bonds with the 1 s hydrogen atoms.

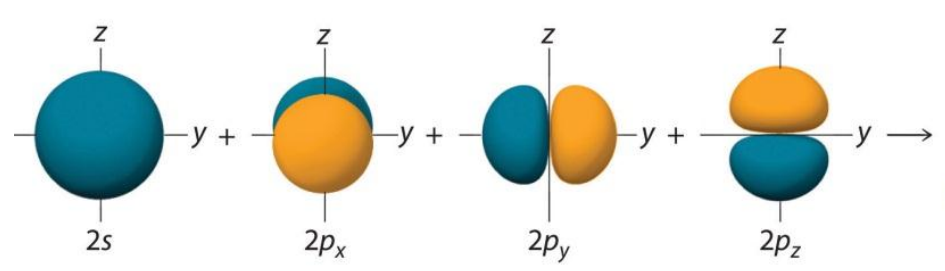

Figure 1.4: Formation of $\mathrm{sp}^{3}$ hybrid orbitals[2]

sp3 hybridization is also exhibited by diamonds. Diamond forms when liquid carbon condenses under high pressure. Diamond lattice consists of two interpenetrating face center cubic (fcc) lattices, with a lattice spacing of $0.357 \mathrm{~nm}$. Although both graphite and diamond share the same atomic ingredient, they are physically extremely different. Graphite is a very soft material due to its layered structure. But diamond is one of the hardest natural materials because all bonds are covalent $\sigma$ bonds. All 4 valence electrons in the outer atomic shell are used in the formation of the $\sigma$ bonds. This makes diamond a good insulator with large band gap of $5.47 \mathrm{eV}$. Electrons in the 
weaker $\pi$ bonds in graphite are delocalized which leads to a good electronic conduction properties.

\subsection{History of graphene}

Graphene is a known material for the scientific community for decades. In 1947, Canadian theoretical physicist Philip Wallace predicted the extraordinary electronic properties of graphene[3]. For years, graphene was considered as a material for academic purposes and existed only in theory and presumed not to exist as a free standing material. A. Geim, K. Novoselov, and co-workers were among the first to successfully obtain the elusive free-standing graphene films. This was a remarkable achievement. 2010 Nobel Prize for Physics was awarded to Geim and Novoselov for "groundbreaking experiments regarding the two-dimensional material graphene".

Graphene is a two dimensional honeycomb lattice with monolayer of carbon atoms and is considered being the basic structure of graphitic materials with different dimensionalities. Fullerene is a zero dimension structure and can be formed by wrapping a graphene layer. Graphene can be rolled to form one dimensional nanotube while stacked graphene can form three dimensional graphite. 


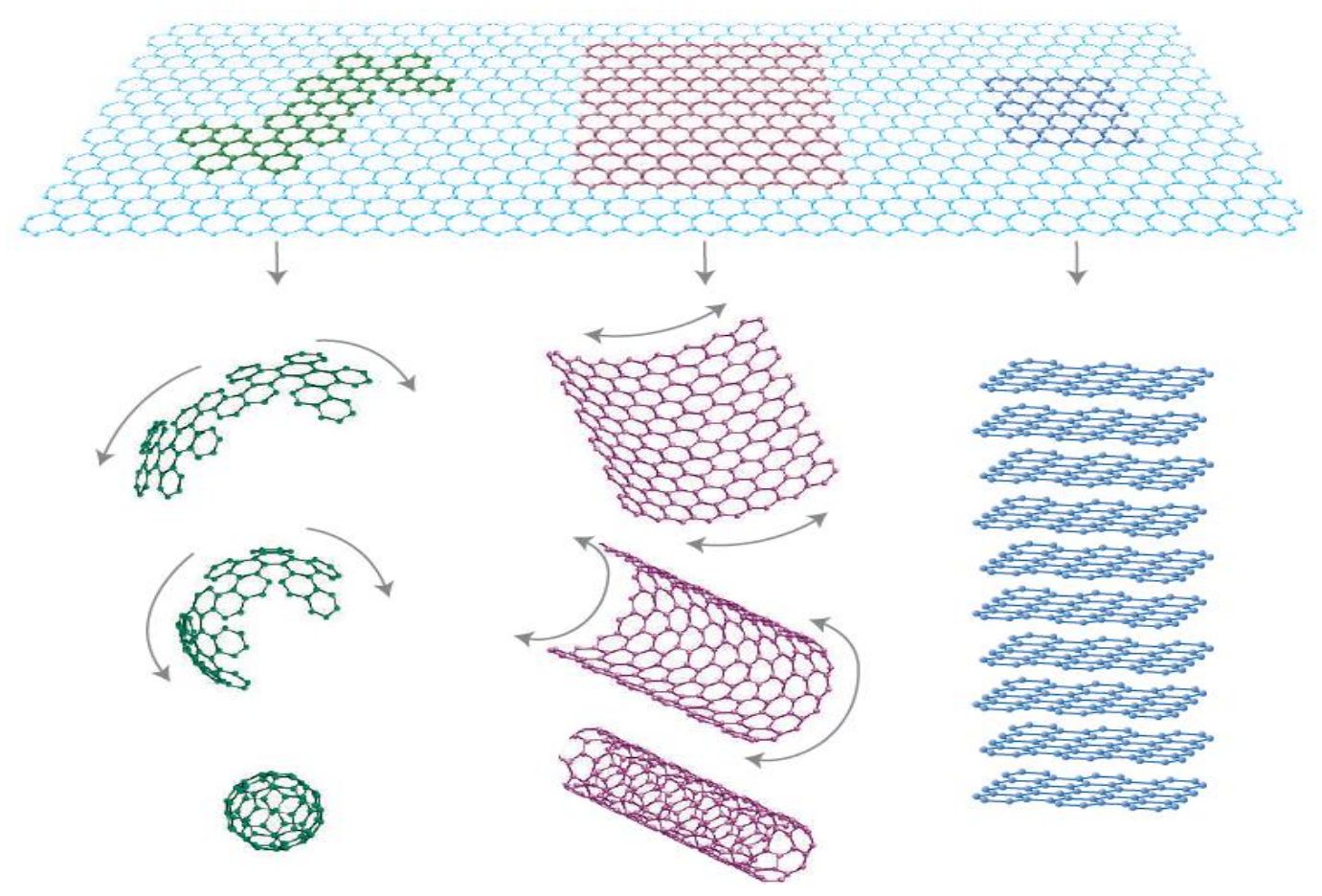

Figure 1.5: Graphene as the 2D building material for carbon materials of all other dimensionalities. It can be wrapped up into OD buckyballs, rolled into 1D nanotubes or stacked into 3D graphite[4].

Graphene has been studied theoretically for the past sixty years and can be used for describing different carbon based material properties. After forty years of studies scientists found that graphene is also an excellent condensed matter, which obeys the $(2+1)$ dimensional quantum electrodynamics. This propelled the graphene into a successful theoretical model. Although graphene known as the integral part of 3D carbon based materials it was predicted not to be in a free state. But free standing graphene found unexpectedly three years ago $[4,5]$. Follow up experiments done on graphene confirmed that its charge carriers were indeed massless Dirac fermions. This property attracted researchers towards graphene.

More than 70 years ago, Landau and Peierls predicted that two dimensional crystals are thermodynamically unstable and could not exist[6, 7]. According to their 
theory, divergent contribution of thermal fluctuations in low dimensional crystal lattices should lead to displacements comparable to their interatomic distances. This theory was later supported by experimental observations. Melting point of thin films decreases with decreasing thickness, which leads the film to become unstable. For this reason, atomic monolayers have known only as an integral part of larger 3D structure.

Experimental discovery of graphene and other free standing two dimensional atomic crystals lead researchers to obtain crystals on top of non crystalline substrates or in liquid suspension or as suspended membranes.

A single atomic plane is considered as a $2 \mathrm{D}$ crystal and large number of layers should be considered as a thin film of the 3D material. In the case of graphene, 10 to 20 layers are considered as the 3D the limit [8] because electronic structure rapidly evolves with the number of graphene layers. Both mono layer and bilayer graphene are considered to be a zero band gap material. They can also be referred to as zero overlap semimetals. But for three or more layers, the spectra become increasingly complicated.

\subsection{Band structure of Graphene}

The most popular method of analyzing graphene band structure is the tight binding method. This was first introduced by Wallace[3]. The band structure of graphene exhibits very unique features. The first two bands do not have a gap or they do not overlap each other. These two bands are intersecting in two inequivalent points. These points are known as Dirac points in the first Brillouin zone. For undoped graphene, Fermi level lies exactly at the intersection points (Dirac point). Graphene is a zero band gap semiconductor. 


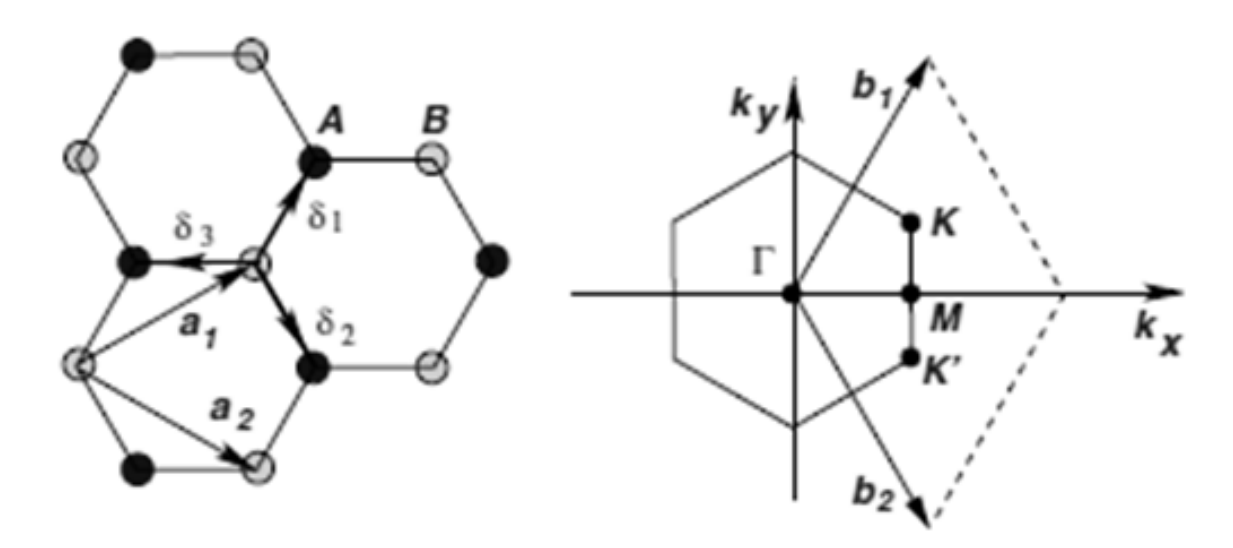

(a)

(b)

Figure 1.6: Graphene hexagonal lattice structure and the corresponding Brillouin zone. (a) $a_{1}$ and $a_{2}$ are the lattice vectors. There are two carbon atoms ( 1 and 2 ) in one unite cell. (b) the reciprocal lattice of graphene is defined by $b_{1}$ and $b_{2}$. The first Brillouin zone is shown as the hexagonal

Figure 1.6a shows a Graphene hexagonal lattice constructed as a superposition of two triangular lattices A and B, with basis vectors $\boldsymbol{a}_{\boldsymbol{i}}$ for lattice A and vectors $\boldsymbol{\delta}_{\boldsymbol{i}}$ with $\mathrm{i}=1,2,3$ 1,2 $\mathrm{i}$ connecting A to B. Figure $1.6 \mathrm{~b}$ shows a corresponding Brillouin zone. The Dirac cones are located at the $\mathrm{K}$ and $\mathrm{K}$ ' points.

Here the $\boldsymbol{a}_{1}$ and $\boldsymbol{a}_{\mathbf{2}}$ are given by,

$$
\overrightarrow{a_{1}}=(\sqrt{3} / 2,1 / 2), \quad \overrightarrow{a_{2}}=(\sqrt{3} / 2,-1 / 2)
$$

and, 


$$
\begin{gathered}
\vec{\delta}_{1}=(1 / 2 \sqrt{3}, 1 / 2) \\
\vec{\delta}_{2}=(1 / 2 \sqrt{3},-1 / 2) \\
\vec{\delta}_{3}=(-1 / \sqrt{3}, 0)
\end{gathered}
$$

By taking the unit cell area as,

$$
A=a^{2} \sin (\pi / 3)=\sqrt{3} / 2
$$

Reciprocal lattice can be written as,

$$
\begin{aligned}
& \vec{b}_{1}=2 \pi \frac{\vec{a}_{2} \times \hat{z}}{A}=2 \pi(1 / \sqrt{3}, 1) \\
& \vec{b}_{2}=2 \pi \frac{\vec{a}_{1} \times \hat{z}}{A}=2 \pi(1 / \sqrt{3},-1)
\end{aligned}
$$

Graphene has two carbon atoms per unit cell. Every carbon atom has four valence electrons, of which three are used for chemical bonds in graphene plane $(\sigma$ bonds). The fourth electron is in a $2 p_{x}$ orbital which oriented perpendicular to the plane. Since the $\sigma$ bonds are localized and not contributing to the electronic conduction, the only concern is the energy band structure of the fourth electron ( $\pi$ band). There are two such electrons in one unit-cell, therefore there should be two $\pi$ bands, $\pi$ and $\pi^{*}$, with $\pi$ corresponding to valence band (bonding) and $\pi^{*}$ corresponding to conduction band (anti-bonding) 
The tight binding approach to electronic band structure is one of the standard methods of condensed matter physics. The starting point is to assume a basis set of localized orbitals on each site of an atomic structure. The simplest model consists under the assumption that only one localized orbital is important at each site. This is also called single band model. Some crystal structures, such as the honeycomb or fcc lattices have more than one site per unit cell. This will require a slightly more complex treatment. Bloch's theorem states that the wave function for an electron is,

$$
\psi=e^{i k \cdot R} \phi(\vec{r}-\vec{R})
$$

where, $\vec{R}$ is the set of lattice vectors defined by linear combinations of $\boldsymbol{a}_{1}$ and $\boldsymbol{a}_{2} . \phi(r)$ is a slow-varying envelope function to be calculated. In the framework of the tight-binding method, the assumption that must be made is that the atomic wave function is well localized at the position of the atoms. When the overlap between neighboring atomic wave functions, though nonzero, is very small, $\phi$ can be approximated by a linear combination of a set of atomic functions. This is why the tight-binding method is known as the linear combination of atomic orbitals (LCAO). In the case of graphene, consider only the $2 \mathrm{px}$ orbitals. There are two such orbitals per unit cell, which shall call $\phi_{1}$ and $\phi_{2}$ with 1 and 2 referring to the two carbon atoms in one unit cell. Then the total wave function can be written as,

$$
\phi=b_{1} \phi_{1}+b_{2} \phi_{2}
$$

Where, $\mathrm{b}_{1}$ and $\mathrm{b}_{2}$ are constants and $\left|b_{1}\right|^{2}+\left|b_{2}\right|^{2}=1$. Let's consider a single electron which experiences the atomic potential given by all the carbon atoms. This 
Hamiltonian is given by,

$$
\begin{array}{r}
H=-\frac{\nabla^{2}}{2 m}+\sum_{R}\left(V_{\text {atom }}\left(\vec{r}-\overrightarrow{r_{1}}-\vec{R}\right)\right. \\
\left.+V_{\text {atom }}\left(\vec{r}-\overrightarrow{r_{2}}-\vec{R}\right)\right)
\end{array}
$$

Where $\overrightarrow{r_{1}}$ and $\overrightarrow{r_{2}}$ are the positions of the two atoms in the unit cell given in Figure 1.6. $\boldsymbol{b}_{1}$ and $\boldsymbol{b}_{2}$ can be obtained by solving the Schrödinger equation.

$$
H \psi=E \psi
$$

There are two equations for two parameters, $b_{1}$ and $b_{2}$. Considering two states $\phi_{1}$ and $\phi_{2}$

$$
\left\langle\phi_{j}|H| \psi\right\rangle=E\left\langle\phi_{j} \mid \psi\right\rangle
$$

Where, $j=1,2$

It is important to note that only the nearest neighbor products have to be taken into account. Therefore only need to consider $\vec{R}=0, \vec{a}_{1}, \vec{a}_{2}$ terms in $|\psi\rangle$. Two equations are obtained as a result,

$$
\begin{gathered}
\left\langle\phi_{1} \mid \psi\right\rangle=b_{1}+b_{2}\left\langle\phi_{1} \mid \phi_{2}\right\rangle\left(1+e^{-i k \cdot a_{1}}+e^{-i k \cdot a_{2}}\right) \\
\left\langle\phi_{2} \mid \psi\right\rangle=b_{2}+b_{1}\left\langle\phi_{2} \mid \phi_{1}\right\rangle\left(1+e^{i k \cdot a_{1}}+e^{i k \cdot a_{2}}\right) \\
\text { Here, }\left\langle\phi_{1} \mid \phi_{2}\right\rangle=\left\langle\phi_{2} \mid \phi_{1}\right\rangle
\end{gathered}
$$


The total Hamiltonian $H$ contains the exact atomic Hamiltonian for atom 1.

$$
H_{1}=-\frac{\nabla^{2}}{2 m}+V_{a t o m}\left(\vec{r}-\overrightarrow{r_{1}}\right)
$$

Potential from all other atoms which is simply denoted by $\Delta H_{1}$. Likewise for the atom 2 corresponding perturbation is $\Delta H_{2}$. Therefore,

$$
H=H_{1}+\Delta H_{1}=H_{2}+\Delta H_{2}
$$

Since $\phi_{j}$ are eigenfunctions of $H_{j}$, we have a simple relation

$$
H\left|\phi_{j}\right\rangle=\epsilon_{j}\left|\phi_{j}\right\rangle
$$

where $\epsilon_{j}$ is the energy of the carbon $2 p_{z}$ orbital. Obviously, $\epsilon_{1}=\epsilon_{2}$, so they will be called $\epsilon_{0}$. Furthermore, the energy can be set to zero, so $\epsilon_{0}=0$.

The results is,

$$
\begin{aligned}
& \left\langle\phi_{1}|H| \psi\right\rangle=\left\langle\phi_{1}\left|H_{1}+\Delta H_{1}\right| \psi\right\rangle=\left\langle\phi_{1}\left|\Delta H_{1}\right| \psi\right\rangle \\
& \left\langle\phi_{2}|H| \psi\right\rangle=\left\langle\phi_{2}\left|H_{2}+\Delta H_{2}\right| \psi\right\rangle=\left\langle\phi_{2}\left|\Delta H_{2}\right| \psi\right\rangle
\end{aligned}
$$

In calculating terms $\left\langle\phi_{j}\left|H_{j}\right| \psi\right\rangle$ only on site and the nearest neighbor overlaps are relevant. After this consideration, there are only a few terms retained

$$
\left\langle\phi_{1}\left|\Delta H_{1}\right| \psi\right\rangle=b_{1} \beta+b_{2} \gamma_{1} f^{*}(k)
$$




$$
\left\langle\phi_{2}\left|\Delta H_{2}\right| \psi\right\rangle=b_{2} \beta+b_{1} \gamma_{1} f^{*}(k)
$$

Here,

$$
\begin{gathered}
\beta=\left\langle\phi_{1}\left|\Delta H_{1}\right| \phi_{1}\right\rangle \\
\gamma_{1}=\left\langle\phi_{1}\left|\Delta H_{1}\right| \phi_{2}\right\rangle=\left\langle\phi_{2}\left|\Delta H_{2}\right| \phi_{1}\right\rangle \\
f(k)=\left(1+e^{i k \cdot a_{1}}+e^{i k \cdot a_{2}}\right)
\end{gathered}
$$

By substituting and transformed into an eigenvalue equation,

$$
\left(\begin{array}{cc}
\beta & f^{*}(k)\left(\gamma_{1}-\gamma_{0} E\right) \\
f(k)\left(\gamma_{1}-\gamma_{0} E\right) & \beta
\end{array}\right)\left(\begin{array}{l}
b_{1} \\
b_{2}
\end{array}\right)=E\left(\begin{array}{l}
b_{1} \\
b_{2}
\end{array}\right)
$$

from which the dispersion relation $E=E(k)$ can be obtained. By writing the Schrödinger equation this way, assume that the two basis wave functions

$$
\psi_{(1,2)}=\sum_{R}\left(e^{i k \cdot R} \phi_{(1,2)}(r-R)\right)
$$

Here $b$ is the energy variation of the $\mathrm{p}_{\mathrm{x}}$ atomic orbital induced by all the other carbon atoms in the graphene plane. It corresponds to a small rigid shift of the energy band, and can be neglected. The dispersion relation can be written simply,

$$
\begin{gathered}
E(k)= \pm \gamma_{1}|f(k)| \\
E(k)= \pm \gamma_{1} \sqrt{1+4 \cos \left(\frac{k_{y} \sqrt{3} a}{2}\right) \cos \left(\frac{k_{x} a}{2}\right)+4 \cos ^{2}\left(\frac{k_{x} a}{2}\right)}
\end{gathered}
$$

where $k_{x}$ and $k_{y}$ are the components of $\mathrm{k}$ in the $(\mathrm{x}, \mathrm{y})$ coordinates 
(a)

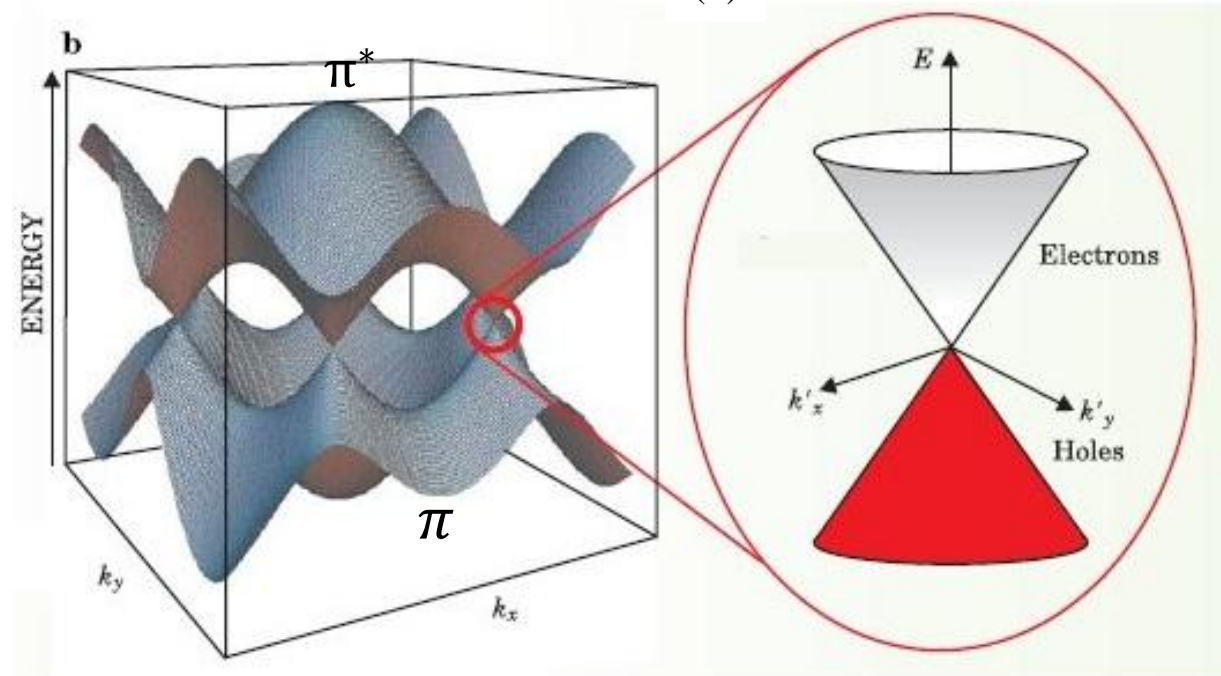

Figure 1.7: Electronic dispersion in the honeycomb lattice. (a) Energy spectrum $\mathrm{t}=2.7 \mathrm{eV}$. (b) Zoom in of the energy bands close to one of the Dirac points.

The plus sign in the energy band equation applies to the upper band $\left(\pi^{*}\right)$ and the minus sign the lower $(\pi)$ band In Figure 1.7a shows the full band structure of graphene. Figure $1.7 \mathrm{~b}$ shows a zoom in view of the band structure close to one of the Dirac points at the $\mathrm{K}$ and $\mathrm{K}$ ' points in the Brillouin zone[9].

As far as the electronic transport is concerned, we are interested in the low energy region just around the $\mathrm{K}, \mathrm{K}^{\prime}$ points. In this regime, the Hamiltonian can be approximated by its first order expansion. First, look at $\mathrm{K}$ point, where $\mathrm{k}=\kappa+\mathrm{K}$, and $\mathrm{E}(\mathrm{k})=\epsilon \kappa+E(\mathrm{k})$. by expanding $f(\mathrm{k})$ to its first order, eigenvalue equation can be reduced to,

$$
-\frac{\sqrt{3} \gamma_{1} a}{2}\left(\begin{array}{cc}
0 & k_{x}-i k_{y} \\
k_{x}+i k_{y} & 0
\end{array}\right)\left(\begin{array}{l}
b_{1} \\
b_{2}
\end{array}\right)=\epsilon(k)\left(\begin{array}{l}
b_{1} \\
b_{2}
\end{array}\right)
$$

The dispersion relation can be written as, 


$$
\epsilon(k)= \pm h v_{F}|k|
$$

Here $v_{F}$ is the Fermi velocity given by the constant,

$$
v_{F}=\frac{\sqrt{3} \gamma_{1} a}{2 h}
$$

\subsection{Synthesis of graphene}

It has been known since the late 1970's that carbon precipitates in the form of thin graphitic layers on transition metal surfaces[10]. Currently, there are various techniques being used to prepare graphene of various dimensions, shapes and quality. However, not many methods are scalable. It is logical to categorize these by the quality of the resulting graphene.

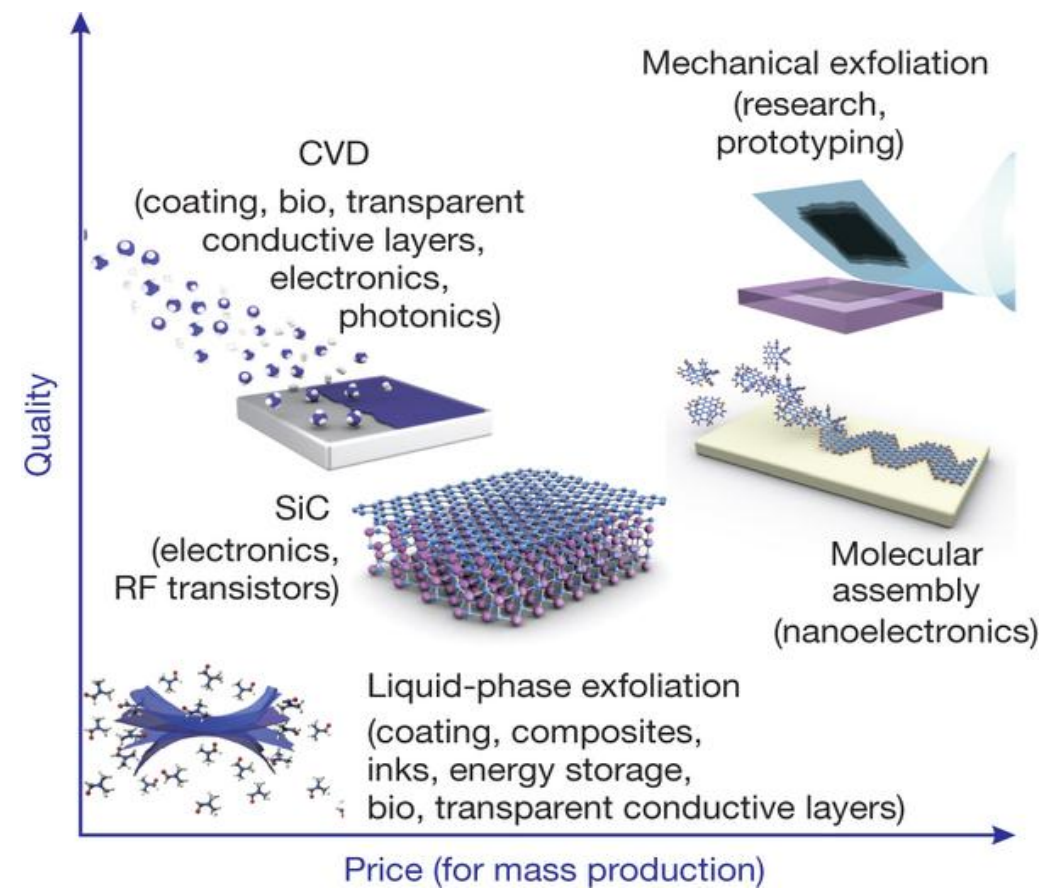

Figure 1.8: Scalable techniques of graphene production. Several techniques are arranged in terms of size, quality and price for any particular application [11]. 


\subsubsection{Mechanical exfoliation}

The first report of isolating graphene onto insulating $\mathrm{SiO}_{2}$ substrate by mechanical exfoliation was made by Geim and co-workers in 2004[5]. This led them to the Nobel prize in physics in 2010. Although carbon precipitation in the form of thin graphitic layers has been known since the late 1970's on transition metal surfaces, no electronic properties have been measured, since the substrates were all metallic and conducting. No techniques were developed at that time to transfer them on to insulating substrates. There have been some reports on thin graphitic flakes by mechanical rubbing on $\mathrm{SiO} 2$ substrates[12]. Still there were no reports on their electrical property characterization. Mechanical exfoliation is perhaps the most unusual and famous method for obtaining single layer graphene flakes on desired substrates. This method produces graphene flakes from (Highly ordered pyrolytic graphite) HOPG by repeated peeling/exfoliation. This mechanical exfoliation can be performed by different method such as scotch tape[5], ultrasonication[13], electric field[14] and even by transfer printing technique $[15,16]$. Transfer printing of macroscopic graphene patterns from patterned HOPG using gold films has been reported recently[17]. Several techniques such as optical microscopy, Raman spectroscopy and AFM used to characterize the mechanically exfoliated graphene. Optical microscopy is a popular technique to locate graphene on substrates like thermally grown $\mathrm{SiO}_{2}$ on $\mathrm{Si}$. Depending on thickness graphene flakes exhibit a characteristic color contrast on a layer of $300 \mathrm{~nm}$ thickness on top of Si wafers[18]. Raman spectroscopy can be used to identify the thickness of graphene flakes and determining its quality. Graphene exhibits characteristic Raman spectra based on number of layers present and crystalline quality [19-21]. The yield of single and few layer graphene obtained by this method is very poor and the flakes are randomly distributed on the substrate. The 
graphene flakes obtained are very small and range from a few microns to a couple of millimeters in size. This technique is not scalable to industrial level, but serves as a good technique to obtain high quality graphene samples for research purposes.

\subsubsection{Epitaxial graphene on silicon carbides}

Graphene is formed epitaxially on (Silicon Carbide) SiC by thermally decomposing $\mathrm{Si}$ atoms from the $\mathrm{SiC}$. This epitaxial growth on $\mathrm{SiC}$ is a very promising method to obtain large scale production of graphene. Formation of epitaxial graphene via the thermal desorption process of $\mathrm{Si}$ from $\mathrm{SiC}$ was pioneered by Claire Berger in 2004 [22]. It is a very attractive method since $\mathrm{SiC}$ wafers up to $100 \mathrm{~mm}$ in diameter are commercially available. $\mathrm{SiC}$ is a wide band gap semiconductor $(\sim 3 \mathrm{eV})$. This enables preforming electrical measurements on graphene while it is on SiC. Growth of epitaxial graphene on $\mathrm{SiC}$ requires UHV conditions. This makes the process difficult and expensive. The quality of epitaxial graphene on $\mathrm{SiC}$ was not up to device levels and still posed many challenges. One way to overcome this challenge is to grow epitaxial graphene at close to atmospheric pressures (900 mbar) in an argon atmosphere. This technique exhibits better crystalline quality, high carrier mobility and Hall conductivity than that of graphene films grown by vacuum graphitization[23]. There are few reports about the mechanisms and kinetics of epitaxial graphene growth on $\operatorname{SiC}[24,25]$. HRTEM reveals nucleation of graphene along (1-10n) plane on the SiC surface steps. Graphene quality can be improved by increasing the temperature of graphitization[26]. Similar HRTEM studies have also been carried out on hexagonal face of 6-H SiC[27]. There are two major downsides to this technique. One is the high cost of the $\mathrm{SiC}$ wafers and the high temperatures used during growth. This will make $\mathrm{SiC}$, not directly compatible with silicon electronics 
technology. Due to the high temperature growth, high substrate cost and smaller diameter wafers, applications of graphene on $\mathrm{SiC}$ will be limited.

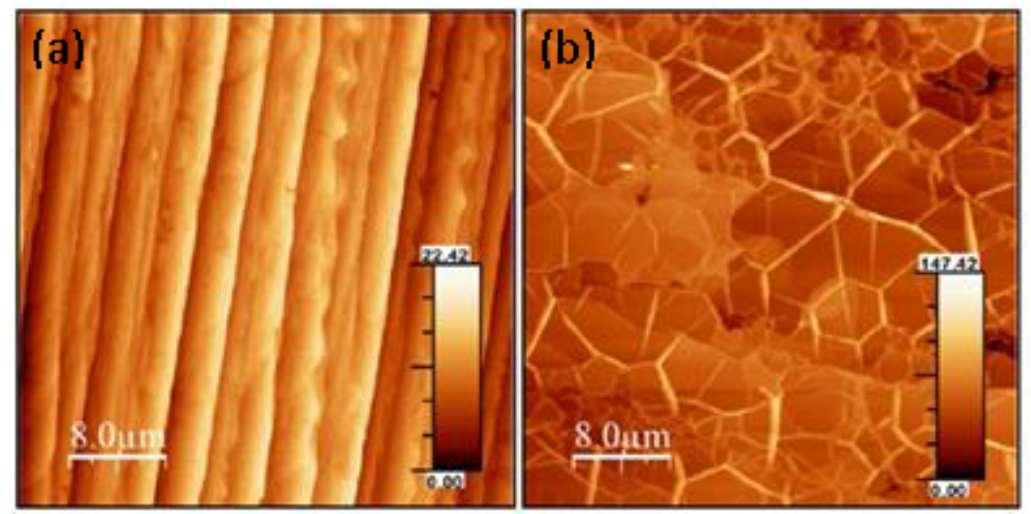

Figure 1.9: Atomic force map of $6 \mathrm{H} \mathrm{SiC}$ after graphene growth. (a) Si-face (b) Cface[28].

\subsubsection{Wet Chemical synthesis of graphene}

Chemical method is another route for large scale graphene synthesis. Main approach of this technique is to produce colloidal suspensions of graphene and chemically modified Graphene from graphite and derivatives of graphite. Graphene produced by these chemical methods are suitable for a variety of applications such as paper like materials [29-33], polymer composites[34], energy storage materials[35], and transparent conductive electrodes[36]. Chemical production of graphene mainly starts with graphene oxide. These methods involve oxidation of graphite in presence of strong acids and oxidants. Graphite oxide is a layered material and highly hydrophilic. So it is easily intercalated with water[37]. Graphite oxide can be easily disintegrated into graphene oxide either by sonication[38] or stirring[39]. This chemically reduced graphene oxide is not pristine graphene. It contains some amount of functionalization groups such as oxygen, hydroxyl groups, epoxy groups etc . Further study must be 
done to understand the structure and reaction mechanisms to produce high quality chemically derived graphene.

\subsection{Band gap engineering of graphene}

Although graphene carries such promising properties right from the first report of its synthesis in transistor applications and touted as a potential replacement for silicon, still it has a major drawback for such applications since it is a zero band gap material. Due to this property, graphene based FETs have low on-off ratios. Thus one of the thrust areas in the field of graphene has been on the techniques to open up a band gap in graphene without compromising on any of its other properties. Various techniques and methods have been devised for this purpose [40].

\subsubsection{Substrate induced band gap opening}
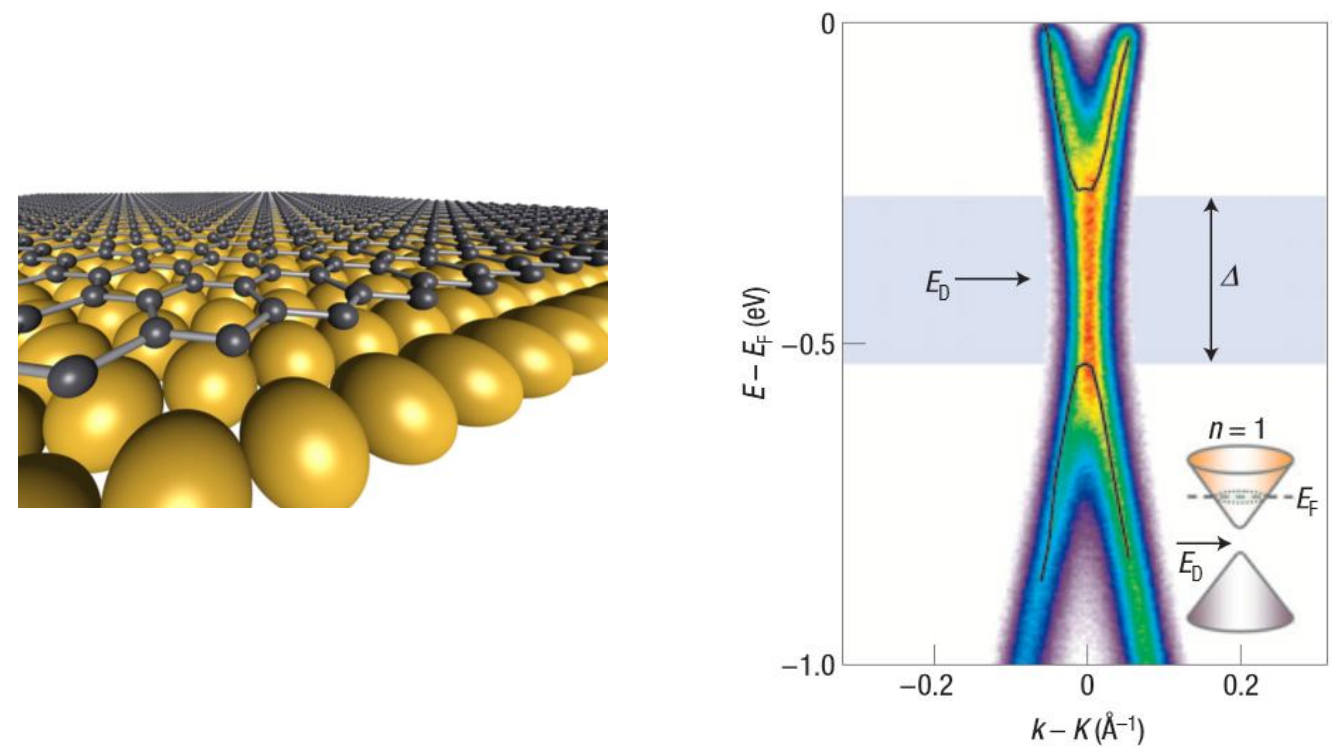

Figure 1.10: Substrate induced band gap opening in graphene on SiC substrate [41] 
The effect of substrate was one of the early techniques to experimentally report band gap opening in graphene. STS/STM reports of band gap opening in epitaxial graphene on epitaxial h-BN have appeared in 2002 even before the first report of graphene isolation on insulating substrate. Epitaxial single layers of h-BN followed by graphene were synthesized on $\mathrm{Ni}$ (111). The graphene/h-BN/Ni(111) system showed a band gap of $0.5 \mathrm{eV}$ in STS measurements thus indicating a substrate induced gap opening[42]. Theoretical studies that performed on h-BN substrate confirm the substrate-induced band gap opening in graphene. The DFT studies indicate that a band gap of $53 \mathrm{meV}$ opens up in graphene on bulk h-BN[43]. Recent study demonstrated that graphene deposited on oxygen terminated $\mathrm{SiO}_{2}$ surfaces can have a band gap of about 0.52 eV[44]. Figure 1.10 shows a band gap opening in epitaxial graphene on $\mathrm{SiC}$ due to interaction with the substrate. Epitaxial graphene on $\mathrm{SiC}$ is electron doped and the Fermi level lies above the gap which makes graphene n-type. Thus in order to make graphene a viable semiconductor either it has to be hole doped or the Fermi level must be moved in between the gap by applying a gate voltage[41]. 


\subsubsection{Chemical substitution doping}
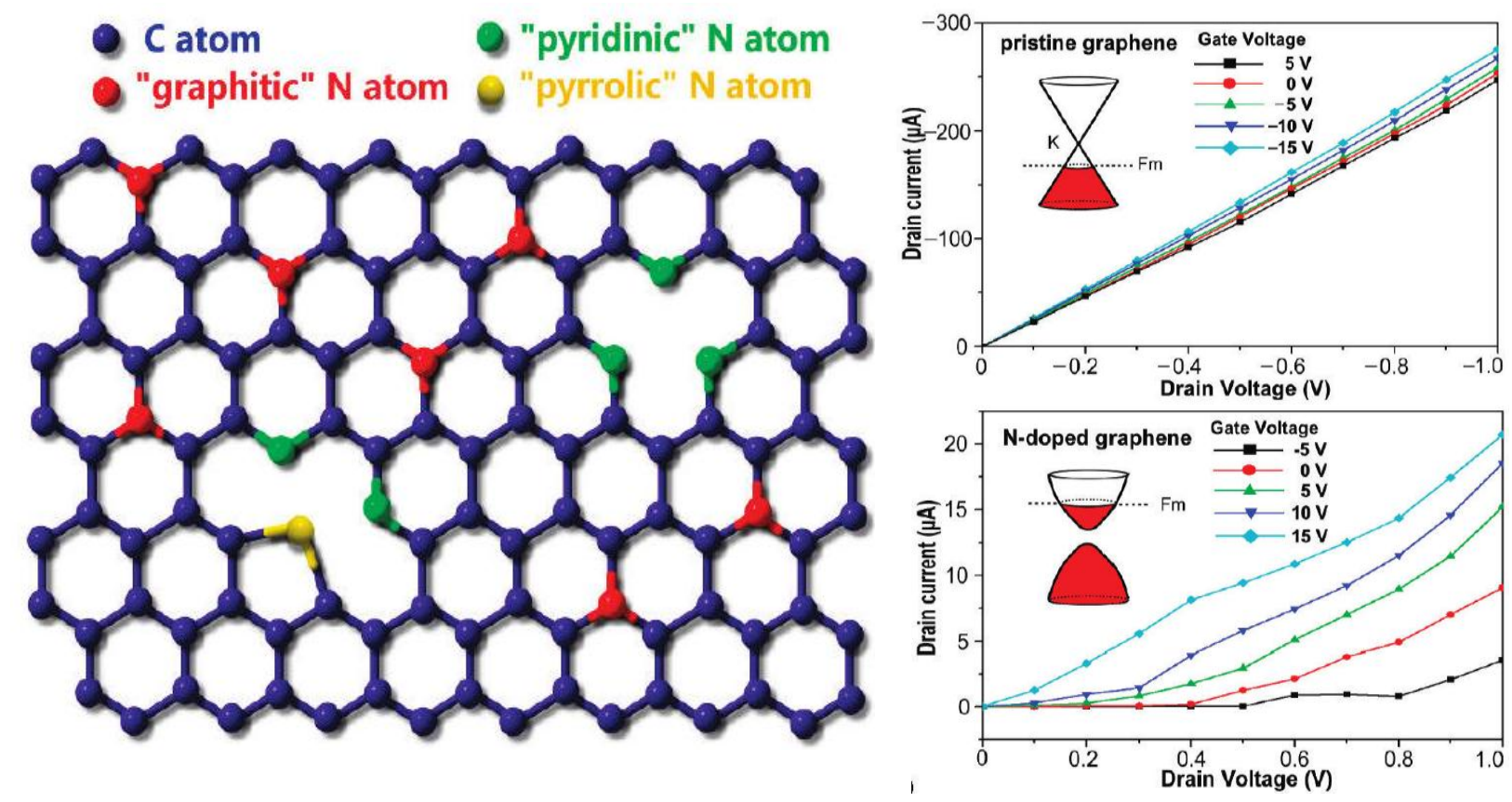

Figure 1.11: Band gap opening by substitutional doping of nitrogen in graphene lattice[45].

Chemical substitution doping method is the most widely used method in doping conventional semiconductors. In a graphitic carbon lattice, both $\mathrm{n}$ and $\mathrm{p}$ type of doping can be achieved by substituting carbon with nitrogen[45-47] or with boron[48, 49]respectively . Compared to the amount of theoretical work that has been done on substitutional doping of graphene so far very few reports have appeared on experimental work verifying the theoretical results. Nitrogen doping has converted graphene into n-type semiconductor. Both experimental and theoretical work has done extensively to demonstrating this. Theoretical studies have shown that $\mathrm{N}$ doping in graphene nanoribbons energetically favors the ribbon edge. Moreover, two dopant atoms prefer to remain as far as possible from each other. Nitrogen doping has been experimentally achieved using CVD by mixing ammonia with methane as the precursor gas on a copper thin film substrate in a recent report which has generated considerable interest. A maximum of $8.9 \%$ nitrogen doping was achieved. It also 
significantly improved the switching ratio in the fabricated FETs. However the carrier mobility was significantly reduced owing to the defects induced by doping[45]. Figure 1.11 shows the doping sites and band structures for the before and after doping. Synthesis of doped graphene by arc discharge method has also been reported. Large quantities of $\mathrm{N}$ doped graphene have been synthesized by DC arc discharge.

In the same way p doping of graphene can be achieved by substitutional doping of boron in the graphitic carbon lattice. Both theoretical and experimental studies of boron doped graphene have already been reported. One study reports that presence of substitutional boron in the graphitic lattice improves the oxidation resistance of the graphitic carbon sample by changing the density distribution of high energy electrons[50]. Boron doping of graphene nanoribbons will lead to transformation of metallic zig-zag nanoribbons to semiconductors. Similar to N doping, substitutionally doped boron atoms also prefer the edge sites energetically. Also substitutionally boron doped GNRs can act as spin filters. Another similar study shows that boron edge doped zig-zag graphene nanoribbons show partial metallic behavior beyond a certain critical electric field thus having potential applications in future spintronic devices. STM and Raman observations also confirm that substitutional doping of boron increases number of defects and disorder in the graphene layers[51]. Carrier mobility and conductivity values of the substitutionally doped graphene are far inferior to that of pristine graphene. But it is one of the most promising ways of opening a band gap in large area free standing graphene.

\subsubsection{Hybrids}

Theoretical studies have appeared on co-doping of graphitic carbon lattice with both boron and nitrogen simultaneously forming $\mathrm{BCN}$ solid solutions in hexagonal lattice 
and synthesis of single layer $\mathrm{BCN}$ semiconductors and $\mathrm{B}_{\mathrm{x}} \mathrm{C}_{\mathrm{y}} \mathrm{N}_{\mathrm{z}}$ nanotubes[52-55]. CVD techniques also enabled to synthesis $\mathrm{BCN}$ films by using $\mathrm{C}, \mathrm{B}$ and $\mathrm{N}$ precursors. However, the thickness of these films has been of the order of a few nanometers to microns. Moreover, these films showed poor crystalline quality [56-58]. All these works report atomic hybrids of $\mathrm{B}, \mathrm{C}$ and $\mathrm{N}$ with semiconducting electronic properties. During the growth process, boron and nitrogen have a tendency to segregate and form $\mathrm{h}-\mathrm{BN}$ and is also energetically favorable. Thus formation of graphene-h- BN hybrids is more feasible. Hybrid C-BN nanotubes with BN domains have been synthesized earlier by laser vaporization, however there was no report on their electronic properties[59]. Same system has been theoretically studied to reveal band gap opening in such hybrid nanotubes. It was also reported that the $\mathrm{N}$ terminated $\mathrm{BN}$ domains were the most energetically stable[60]. Theoretical study of h-BN/GNR/h-BN sandwiched hybrid nanoribbon structures has also been recently reported. This system is structurally stable and that ZGNRs sandwiched between h-BNs behave as half metals and thus have potential applications in spintronics[61]. Large area of 2-3 atomic layer hybrid films consisting of graphene and h-BN domains have been successfully synthesized on $\mathrm{Cu}$ substrates by CVD. By controlling the CVD conditions, these films were also synthesized over varying concentration ranges of $\mathrm{C}$ and $\mathrm{BN}$. Unique dual optical band gap was observed as evidence of band gap opening. These films had isotropic structure and thus the technique looks very promising for synthesizing hybrid films with tunable electronic and optical properties. Theoretical computations carried out in the same study also suggest band gap opening in hybrid films with BN domains and that the band gap increases with decrease in h-BN domain size[62]. Although this method looks very promising, the control over domain size and shape 
has not been achieved yet which is essential for tuning the gap and other electronic properties.

\subsubsection{Graphene nanoribbons}

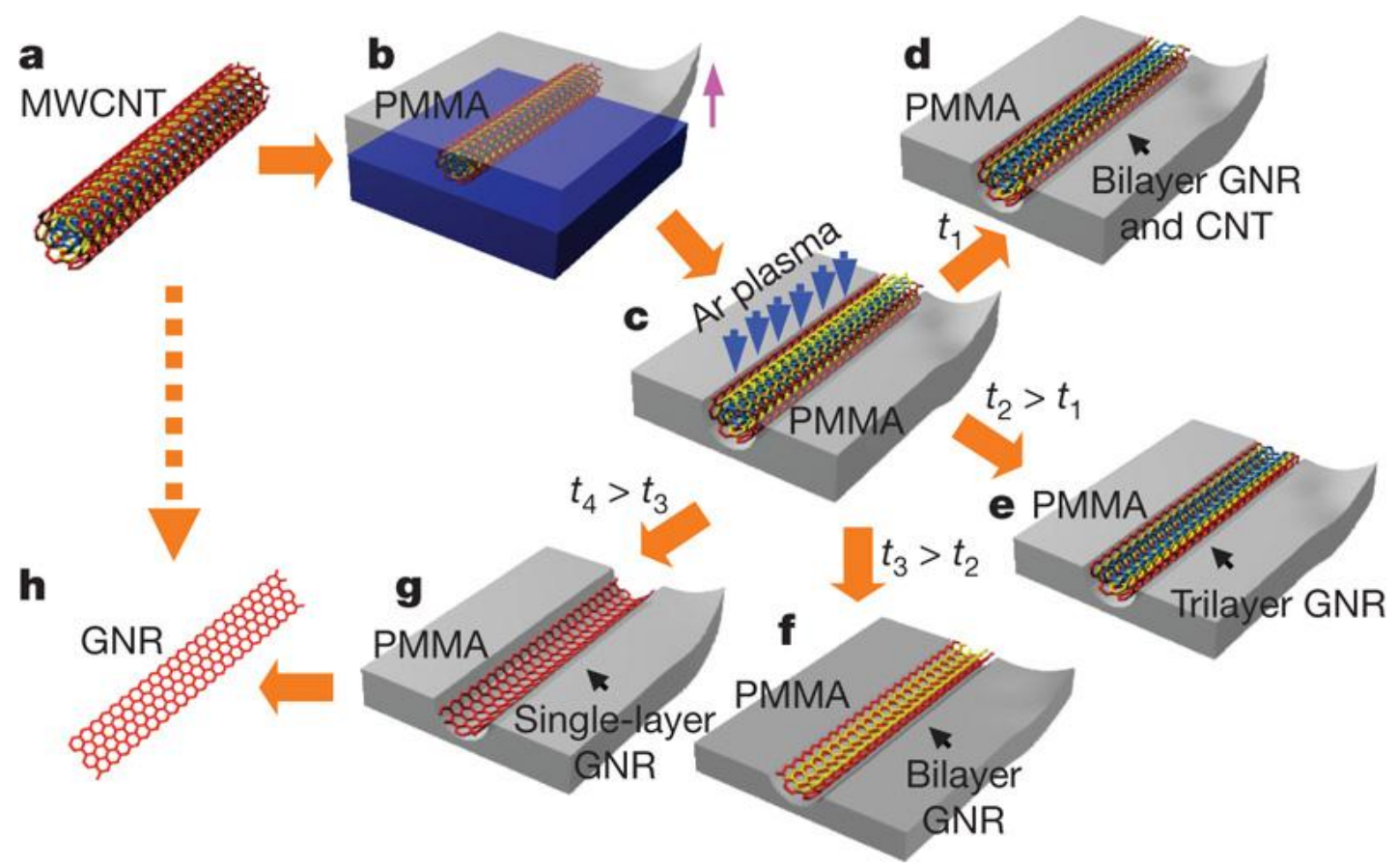

Figure 1.12: Making GNRs from CNTs (a) A pristine MWCNT was used as the starting raw material. (b) The MWCNT was deposited on a Si substrate and then coated with a PMMA film. (c) The PMMA-MWCNT film was peeled from the Si substrate, turned over and then exposed to an Ar plasma. (d-g) Several possible products were generated after etching for different times: GNRs with CNT cores were obtained after etching for a short time $t_{1}(\mathrm{~d})$ tri-, bi- and single-layer GNRs were produced after etching for times $t_{2}, t_{3}$ and $t_{4}$, respectively $\left(t_{4}>t_{3}>t_{2}>t_{1} ; \mathrm{e}-\mathrm{g}\right)$. h, The PMMA was removed to release the GNR[63].

Graphene is a strong candidate for applications in future nanoscale electronics and also as a potential replacement for silicon and even as conductive interconnects in the semiconductor industry. This cannot be achieved unless a finite band gap is opened up in its electronic structure. The above illustrated methods have been successful to a 
certain extent in opening up a finite band gap in graphene and thereby improving onoff ratios in graphene based switches. This can be effectively realized by band gap opening through quantum confinement of electrons in graphene in the form of graphene nanoribbons. In large area planar graphene the electrons are confined in 2 dimensions thus its band structure is different from that of three dimensional graphite. However by making a quasi 1-dimensional nanoribbon out of a large area 2-D graphene sheet would confine the electrons in a single dimension thereby expanding the electronic structure further and making GNRs finite band gap semiconductors similar to semiconducting SWNTs. The graphene ribbons were originally introduced as a theoretical model by Mitsutaka Fujita and co-authors. They examined the edge and nanoscale size effect in graphene[64-66]. Since no impurities are introduced in the graphitic lattice no charge scattering would occur except at the edges and thus the carrier mobility will not be tremendously affected in a nanoribbon. Graphene nanoribbons with width $<10 \mathrm{~nm}$ posses a finite band gap and also have enough carrier mobility thus facilitating high switching ratios. Electronic confinement, coherence and Dirac nature of the carriers in graphene was demonstrated in nanoribbon geometry[67]. This can also be achieved in quantum dot geometry too[68]. So the all semiconducting property of sub $10 \mathrm{~nm}$ GNRs could then bypass the problem of extreme chirality dependence of electronic properties as observed in SWNTs and thus make GNRs an ideal candidate for nanoelectronics. Metallic GNRs are also ideal candidates as conductive interconnects than CNTs since they have a high current carrying capacity. However, controlled synthesis of GNRs in large quantities with narrow length and width distribution is very difficult at the moment. Carbon nanotubes can be formed by rolling up graphene sheets, so, unrolling or unzipping nanotubes should produce planar graphene. This technique was first 
reported by Dai et al.[63] and Tour et al [69]. These studies were done by successfully opening up multiwall carbon nanotubes to form graphene nanoribbons. The width of the nanoribbons thus produced depends on the diameter of the precursor nanotubes. This unzipping of nanotubes was achieved by two entirely different methods. Dai et al. first embedded multiwalled CNTs in a PMMA film and then exposed this film to Ar plasma for various times so as to etch out top exposed portions of tube walls thereby opening up the tubes. With increasing amount of exposure times GNRs with reduced thickness were observed.

\subsection{Scaling theory of localization}

It is important to understand the scaling theory and its consequences for a disordered electronic system to understand the phase transition of graphene from a metal to insulator. It has been shown that, by considering a perturbative approach that for a weakly disordered system a scaling function, $\beta$ can me defined as,

$$
\beta=\frac{d \ln g}{d \ln L}
$$

Here $g$ is the conductance and L is the relevant length scale[70],

It predicts the absence of a metallic state for two dimensions. However it should be noted that this theory ignores the effects of electron-electron interaction which plays an important role in real experimental systems showing metal insulator transitions. 


\subsubsection{Localization within scaling theory}

Studies done in 1970's showed that localization problem can be formulated as a scaling analysis of one parameter. They considered a system of non-interacting disordered electron gas[71, 72].

The basis of the scaling theory is to find the conductance of a system of finite size $L$ subjected to perturbation within the boundary conditions. Localization lengths that are smaller than the length scale of the system essentially insensitive to the boundary conditions. Extended states or localized states with a localization length larger than $L$ should be sensitive to changes in the boundary conditions. For a system of length scale $L$, much larger than the mean free path, it takes time $T$ to travel a distance $L$ with diffusion constant $D$. Based on uncertainty principle between time and energy,

$$
\Delta E=\frac{\hbar}{T}=\frac{\hbar D}{L^{2}}
$$

Here, $\Delta E$ is the sensitivity to the boundary conditions. By considering the Einstein relation for conductivity[73],

$$
\sigma=\frac{e^{2}}{2} D \frac{d n}{d E}
$$

Here, the density of states given by,

$$
\frac{d n}{d E}=\frac{d N}{L^{d} d E}
$$

By considering equation 1.32,

$$
\Delta E=\frac{2 \hbar^{2}}{e^{2}} L^{d-2} \sigma \frac{d E}{d N}
$$

By considering the tight bonding model and the square lattice of size of $L \times L$, 


$$
H=-t \sum_{\langle i j\rangle} c_{i}^{\dagger} \cdot c_{i}+\sum_{i} V_{i} n_{i}
$$

Here $t$ is the hopping amplitude from a site to the nearest neighboring sites. $c_{i}^{\dagger}$ is the creation operators and $c_{i}$ is the annihilation operators. Here $V_{i}$ is a site dependent potential that is randomly distributed between $-V$ and $V$ in all the sites. When the length scale is increased from $L$ to $L^{2}$, the new lattice will have $L^{2}$ squares each with length $L$.

\subsubsection{Scaling theory of $\beta$ function}

Conductance $(g)$ has a asymptotic behavior in both limits of weak and strong scattering[70]. The wave functions are extended and the mean free path $l$ is large in comparison to the inverse Fermi wave vector $k_{F}^{-1}$ in a weakly scattering electronic system. The conductivity $(\sigma)$ to leading order in $\left(k_{F} l\right)^{-1}$ is given by,

$$
\sigma=\frac{n e^{2} \tau}{m}=\frac{n e^{2} l}{\hbar k_{F}}
$$

Here, $n$ is the electron density, $\tau=l / v_{F}$ is the relaxation time and $m$ is the effective electron mass.

Conductance of a $d$-dimensional conductor of linear size $L$,

$$
g(L)=\sigma L^{d-2}
$$

This $L$ depends on the microscopic parameters of the system through a single length scale $\xi_{l o c}$, called the localization length in the insulator and the correlation length (or mean free path) in the metal. This is known as the single parameter scaling hypothesis[70] as in the Figure 1.13. Since in the real space these states are far apart (despite their close energies) the hopping amplitude from one state to another is 
exponentially small. In this regime for length scales $\gg \xi_{l o c}$, the conductance is given by,

$$
g(L) \propto \exp \left(-L / \xi_{l o c}\right)
$$

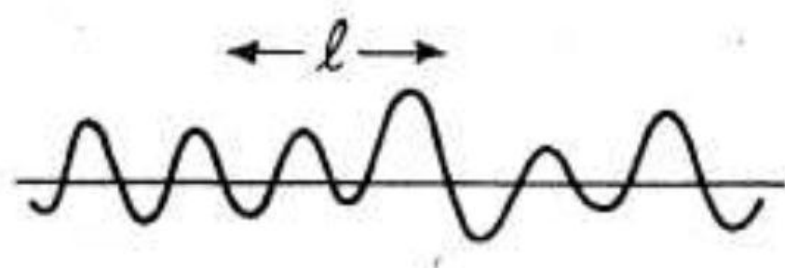

(a)

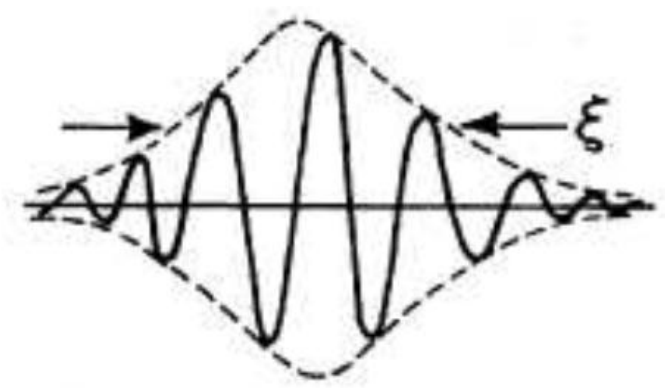

(b)

Figure 1.13: Typical wave function for (a) delocalized (b) localized states, with the mean free path $l$ and the localization length $\xi$ indicated[74].

When considering a particular type of disorder, as the length scale increases from $L$, $g(L)$ will start to change smoothly, starting from $g_{0}$ (conductance at the length scale of the mean free path) and it finally reaches one of the limiting cases of Eq. 1.32 or Eq. 1.33. The final state depends on the microscopic disorder, $g_{0}$ and dimensionality. In $1 \mathrm{D}$ where the localization length $\xi_{l o c}$ is of the order of $l$, all the states are localized and the system does not obey Ohm's law on any length scales.

When considering metal, Ohm's law implies that $g \propto L^{d-2}$. This implies that $g$ depends as a power law on $L$. But in the case of an insulator, conductance decays exponentially, $g \propto L^{-L / \xi}$. In order to interpolate between these two limits, it is convenient to work with the logarithmic derivative.

Function for $\beta$ is given by in terms of conductance $g$ in the equation 1.25 , so scaling behavior of conductance for different dimensions are given by, 


$$
\begin{gathered}
\beta(g)=d-2, \quad g \gg g_{0} \\
=\ln \left(g / g_{0}\right), \quad g \ll g_{0}
\end{gathered}
$$

In the localized regime $\beta(g)$ is negative corresponding to decrease in conductance as $L$ increases. In the limit of weak scattering $\left(\left(k_{F} l\right)^{-1} \ll 1\right)$ the next higher order contribution is the summation of all maximally crossed diagrams [49]. With this correction $\sigma$ is,

$$
\begin{aligned}
& \sigma_{3 D}=\sigma_{0}-\frac{e^{2}}{\hbar \pi^{3}}\left(\frac{1}{l}-\frac{1}{L}\right) \\
& \sigma_{2 D}=\sigma_{0}-\frac{e^{2}}{\hbar \pi^{2}} \ln \left(\frac{l}{L}\right) \\
& \sigma_{3 D}=\sigma_{0}-\frac{e^{2}}{\hbar \pi}(L-l)
\end{aligned}
$$

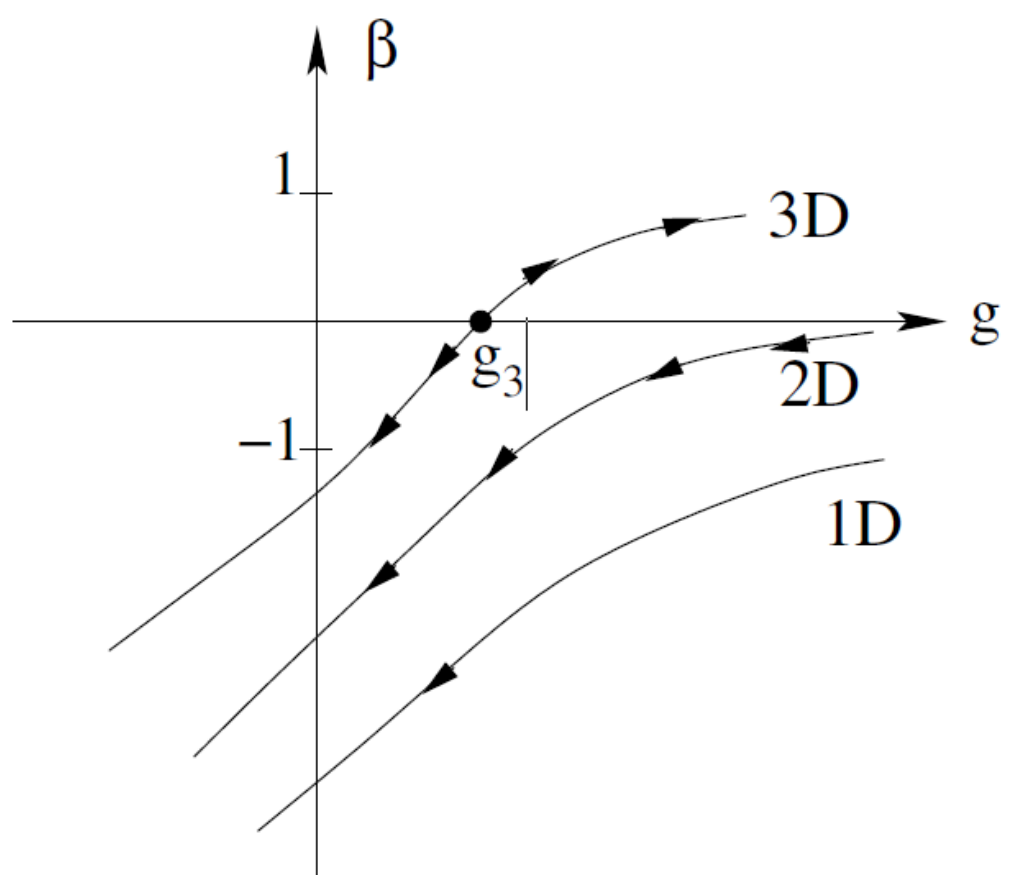

Figure 1.14: Schematic-function for the electrical metal-insulator transition in different dimensions, in the presence of time-reversal symmetry[75, 76].

So $\beta$ function can be written in the form of,

$$
\beta(g)=d-2-\frac{a}{g}
$$


For an electron gas, $a=g_{0}=\pi^{-2}$. This implies that for a disordered system, $\beta(g)$ should always be less than the Ohmic value.

When considering the 3 -D case at very large $g, \beta=1$. But for small $g, \beta$ is negative. For three dimensions, $\beta(g)$ passes through zero at the point $g_{3}$ as shown in Figure 1.14. If the state of the microscopic disorder is such that the conductance $g_{0}$ at length scale $l$ is greater than $g_{3}, \beta(g)$ curve will be in the positive side. With increasing length scale, $\beta(g)$ will increase further. So the system approaches the Ohmic regime. Finally at macroscopic length scales, $\beta$ reaches one. If $g_{0}$ is less than $g_{3}$, curve will be negative and with increasing $\mathrm{L}$, tends to the logarithmic form in the localized regime.

In two dimensions $\beta \leq 0$, therefor at large length scales the system tends to be localized. Consider very weak disorder, with conductance $g_{0}$ on the length scale $L$. Upon increasing the length scale $L, g$ decreases and consequently curve moves downward in the scaling curve until it reaches $\beta \approx \ln \left(g / g_{0}\right)$. One can estimate the localization length from the perturbative value of $\beta$. Conductance at the length scale $L$ is given by,

$$
\begin{gathered}
g(L)=g_{0}-\left(e^{2} / \hbar \pi^{2}\right) \ln (l / L) \\
\text { Here, } g_{0}=\left(e^{2} / 2 \pi \hbar\right)\left(k_{F} l\right)
\end{gathered}
$$

Conductance at the length scale of localization length is zero, so $g_{0}$ can be written as, 


$$
\begin{aligned}
& g_{0}=-\left(e^{2} / \hbar \pi^{2}\right) \ln \left(\xi_{l o c}^{2 D} / l\right) \\
& \text { Here, } \xi_{l o c}^{2 D} \approx l \exp \left(\pi k_{F} l / 2\right)
\end{aligned}
$$

Since the localization length depends on $l$ exponentially, it can be difficult to observe localization in $2 \mathrm{D}$. In the presence of an arbitrary small disorder a $2 \mathrm{D}$ electronic system has non-ohmic behavior on the entire range of the length scales. The $\beta$ function shown in Figure 1.14 is obtained in the absence of spin-orbit coupling. In the presence spin-orbit coupling the function can be positive, leading to a metallic phase in 2D[77].

One dimension $\beta$ always remains negative and it decreases further with increasing the length scale. All states are localized due to repeated backscattering and the localization length is of the order of backscattering mean free path. 


\section{CHAPTER 02}

\section{CHEMICAL VAPOR DEPOSITION OF GRAPHENE: SYNTHESIS AND CHARACTERIZATION}

\subsection{Introduction}

Since graphene was isolated by mechanical exfoliation in 2004, there have been many processes developed to produce few-to-single layer graphene. Producing samples with high carrier mobility and with low density of defects are primary concerns in graphene synthesis. CVD synthesis of graphene enables researches to synthesis large areas of graphene. CVD is a technique that deposit thin films on substrates by chemical reactions. CVD is a very old and known process. Historical reports indicate that CVD technique was developed in the 1880s in the production of incandescent lamps to improve the strength of filaments by coating them with carbon or metal. CVD stated expanding rapidly at the end of the World War II, as researchers realized its advantages for the production of coatings and freestanding shapes. Its importance has been growing ever since. Chemical vapor deposition is a synthesis process in which the chemical constituents react in the vapor phase near or on a heated substrate to form a solid deposit. Numerous chemical processes like thermal decomposition (pyrolysis), reduction, hydrolysis, disproportionation, oxidation, carburization, and nitridation undergo during a CVD process. They can be used either singly or in combination for a complete CVD process. 


\subsection{Kinetics and mass transport mechanisms of CVD synthesis}

Mass transport mechanism is very important in designing a CVD system. This analysis involves what to expect from the reactants as they reach the deposition surface at a given temperature and how do these reactants reach that deposition surface. Any CVD process is subjected to complicated fluid dynamics. Combination of gases is forced through different stages of the CVD system while large variations in temperature and lesser degree of pressure before it comes in contact with the substrate where the deposition reaction takes place. In some cases reaction can take place even before gas reaches the substrate.

Transport kinetics of gas species tend to be complicated during a successful CVD process. Convection and diffusion are the dominating mechanisms in different regions of a reactor. The chemical reaction gets more complicated when it involves many intermediate steps to complete the growth mechanism. Due to the stagnant boundary layer, diffusion of reactive species to or from the substrates is also complicated. This can result a non-uniform thickness film on the fixed substrate surface based on the fluid dynamics as in Figure 2.1. 
Main flow of reactant gases

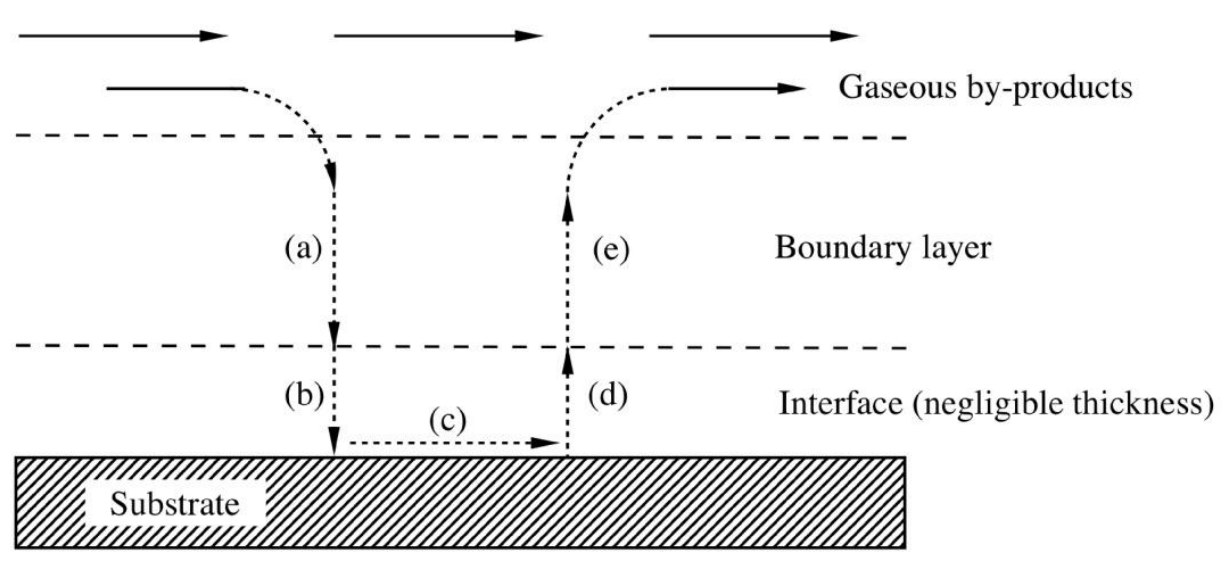

Figure 2.1: Sequence of events during CVD: (a) diffusion of reactants through boundary layer, (b) adsorption of reactants on substrate, (c) chemical reaction takes place, (d) desorption of adsorbed species, and (e) diffusion out of by-products through boundary layer[78].

Depletion of reactants from one end of the reactor to the other end is another important factor of a successful growth. Reaction tends to occur near the front end of the reactor than the end. By increasing the temperatures moving away from the front end of the reactor can eliminate this problem. All the factors in the CVD process are equally important and entangled with each other. It is important to balance all these factors before a successful deposition of thin film with desired thickness and quality.

The behavior of the gas that flows down the tube is govern by fluid mechanics. Reynolds number $\left(\mathrm{R}_{\mathrm{e}}\right)$ is a dimensionless parameter that characterizes the flow of a fluid. This holds if the gas flow is generally laminar. But in real CVD system this laminar flow will be disturbed by convective gas motion and then become turbulent. Velocity of the gas at the deposition surface during a laminar flow is zero. Boundary layer has zero flow velocity the wall and main flow velocity toward bulk gas flow. This boundary layer starts at the inlet of the tube and increases in thickness 
until the flow becomes stabilized. Reactant gases diffuse through the boundary layer to reach the deposition surface.

The thickness of the boundary layer $(\Delta)$ can be interpreted in terms of mass density $(\rho)$, flow density $(u)$, distance from inlet in flow direction $(x)$, viscosity $(\mu)$.

$$
\Delta=\sqrt{\frac{x}{R_{e}}} \quad \text { where, } R_{e}=\frac{\rho u_{x}}{\mu}
$$

This means that the thickness of the boundary layer increases with lower gas flow velocity and with increased distance from the tube inlet.

Consider an approximate behavior of the gas-flow inside a tube. Thermal diffusion is keeping the gas particles away from the hot surface where a steep temperature gradient exists. Figure 2.2 shows a typical velocity pattern in a horizontal tube. Velocity gradient is shallow at the entrance of the tube and increases gradually toward downstream. Steep velocity gradient is noticeable going from maximum velocity at the center of the tube to zero velocity at the surface of the wall.

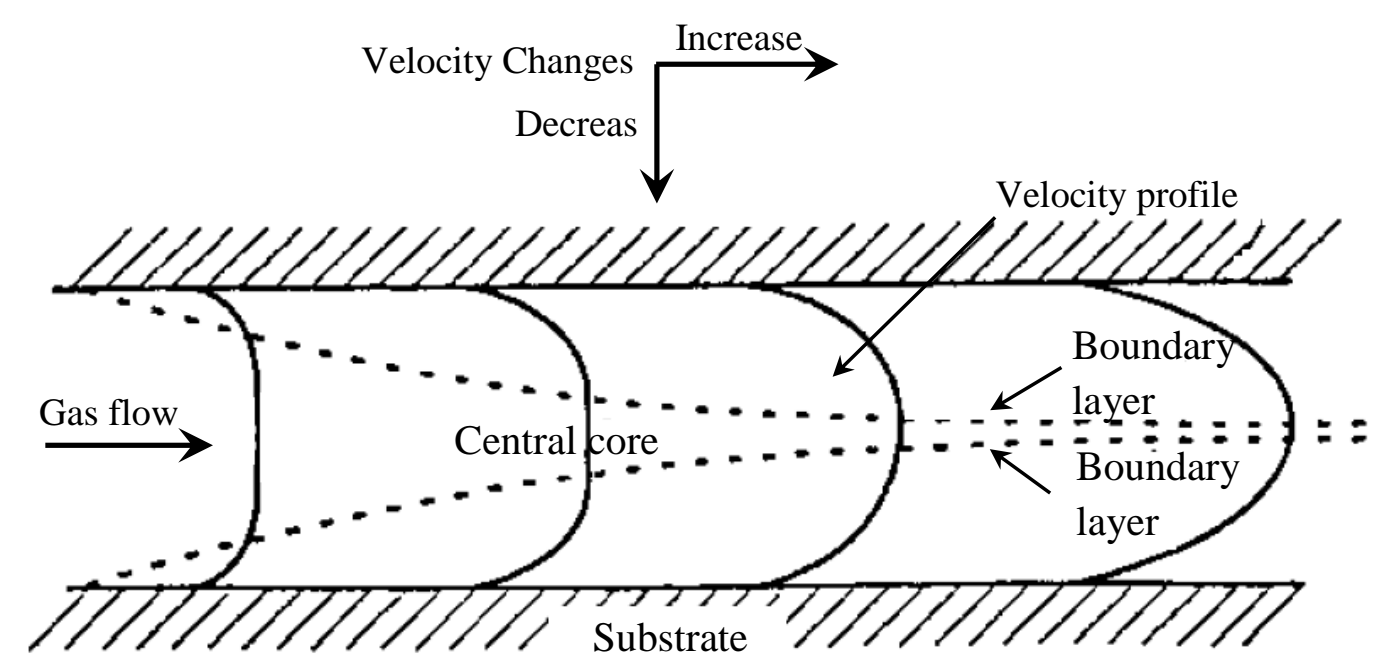

Figure 2.2: Boundary layer and velocity changes in a tube reactor, showing the graphs of velocity recorded at different positions on the tube[78]. 
Figure 2.3 shows a temperature profile of a gas flowing tube. The temperature boundary layer is similar to the velocity layer. The flowing gases through the tube heat rapidly as they come in contact with the hot surface. This will result in a steep temperature gradient. The average temperature increases toward downstream.

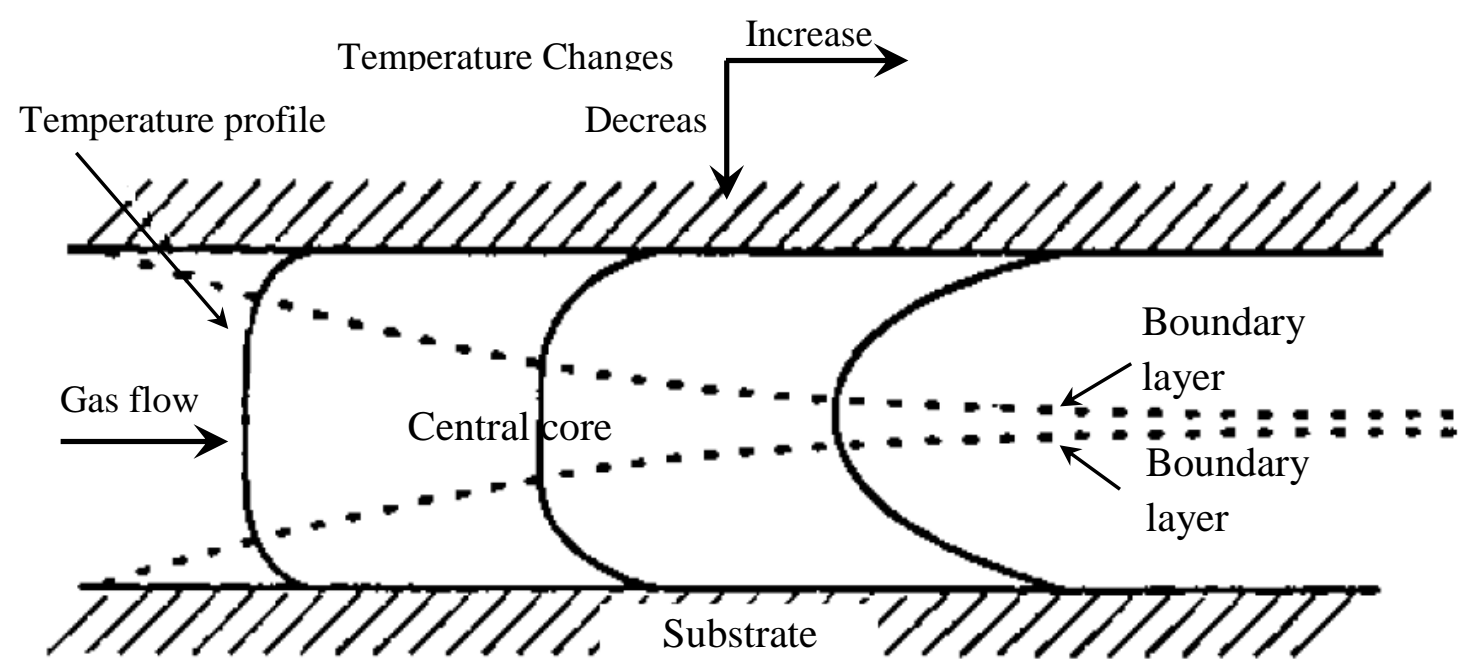

Figure 2.3: Temperature boundary layer and temperature changes in a tubular reactor, showing the graphs of temperature recorded at different positions on the tube[78].

(a) Reactant-Gas Concentration

As the gases flow down the tube, reactance depletes and deposited on the substrate. This will increase the concentration of the by-product gas in the boundary layer. Due to this process, downstream deposition will reduce. The boundary layers are influenced by these three variables (gas velocity, temperature, and concentration) interacting each other. Velocity and temperature profiles are fully developed at an early stage while the deposition reaction is spread far downstream.

The chemical reaction plays an important role in the synthesis process. A CVD system is mainly composed with a gas delivery system, a reactor and a gas removal system as shown in Figure 2.4. Reactive gas species are fed into the reactor during the CVD process by the gas delivery system. Mass Flow Controllers (MFCs) 
are used to manipulate the flow rates of the gases passing through. It is important to mix gases uniformly before they are let in to the reactor. Chemical reaction takes place and the solid materials are deposited on substrates in the reactor. Eventually the by-products of the reaction and non-reacted gases are removed by the gas removal system.

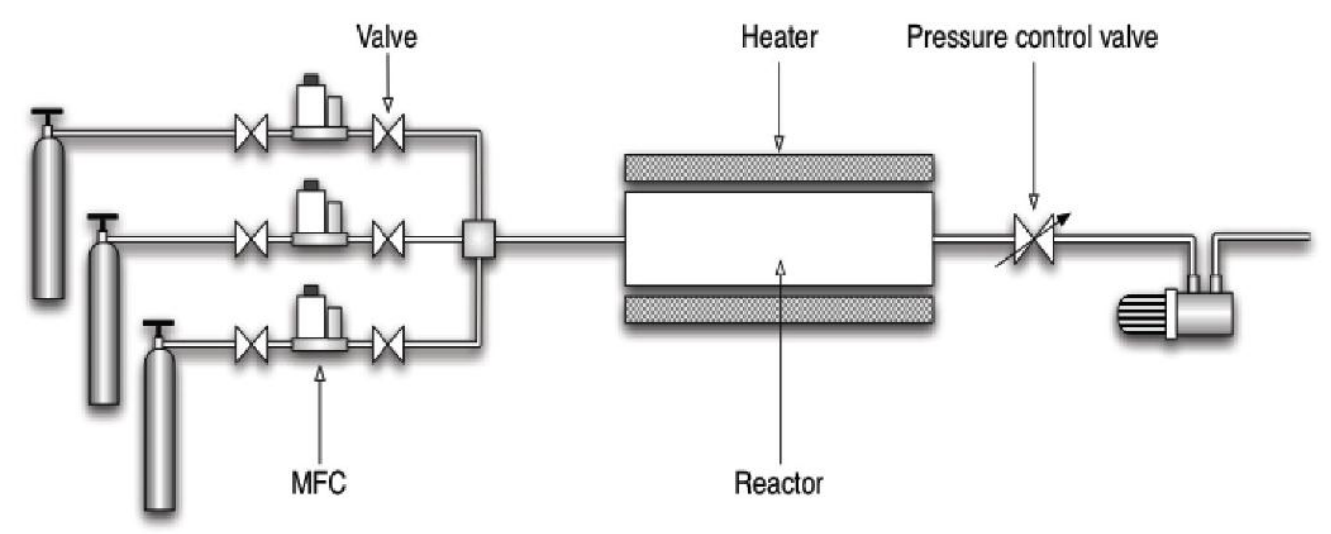

Figure 2.4: Schematic of a CVD system[79].

Precursor dissociation for graphene synthesis should be done only on the substrate surface. This will avoid the precipitation of carbon clusters in the gas phase which is typically in the form of carbon soot and sits on synthesized graphene. Transition metals are typically used as catalysts to achieve the even distribution and decomposition of precursors on surface. Metallic substrates that are in the form of thin foils or films are used in CVD growth of graphene. Since the substrates are in the form of thin foils/films they are poly crystalline in nature and hence the individual grains/crystals are randomly oriented. Carbon segregation also occurs at grain boundaries which lead to folds or areas of higher thickness on the graphene films. CVD growth of graphene has been practiced on both copper[80, 81] and nickel[82, 83] substrates. Growth mechanisms on each of these metals are different from each other. 
In order to form large area graphitic structures without catalysis, temperature is raised to beyond $2500{ }^{\circ} \mathrm{C}$. This temperature is too high and requires special setup for the deposition system and the substrate. The introduction of catalysts lowers energy barriers not only for the pyrolysis of precursors, but also for the graphitic structure formation. Metal catalysts are used for graphene synthesis. These metals have finite carbon solubility. $\mathrm{Cu}$ has almost zero carbon solubility[84] and $\mathrm{Ni}$ has a nonnegligible carbon solubility. At high temperatures during the growth, dissociated carbon atoms on the catalyst surface may dissolve into the bulk due to the finite solubility. Things become worse when these dissolved carbon atoms precipitate back onto $\mathrm{Ni}$ surface as temperature drops and hence unwanted carbon deposition may occur from bottom. Different cooling rates will allow growing different thickness of graphene. Figure 2.5 shows the schematic drawing of graphene grown on $\mathrm{Ni}$ with different cooling rate[85]. Extreme fast cooling will lead to too little carbon precipitation due to the lack of time for carbon to precipitate. Medium cooling is ideal for the graphene synthesis. Slow cooling will not grow anything on the surface and carbon atoms will diffuse deep into the bulk catalyst.

There are other issues associated with catalysts. One is the surface roughness, mainly due to the grain boundaries. Grain bounties have higher surface energy. Carbon prefers to stay in these areas with higher surface energies. These areas have more atomic dangling bonds that could easily attract precipitated carbon atoms. This can lead for deposited graphene to exhibit non-uniformity with thick graphene around grain boundaries. This can be minimized by pre-annealing the catalyst substrates. Annealing will reduce the total length of grain boundaries and other minor surface defects. 


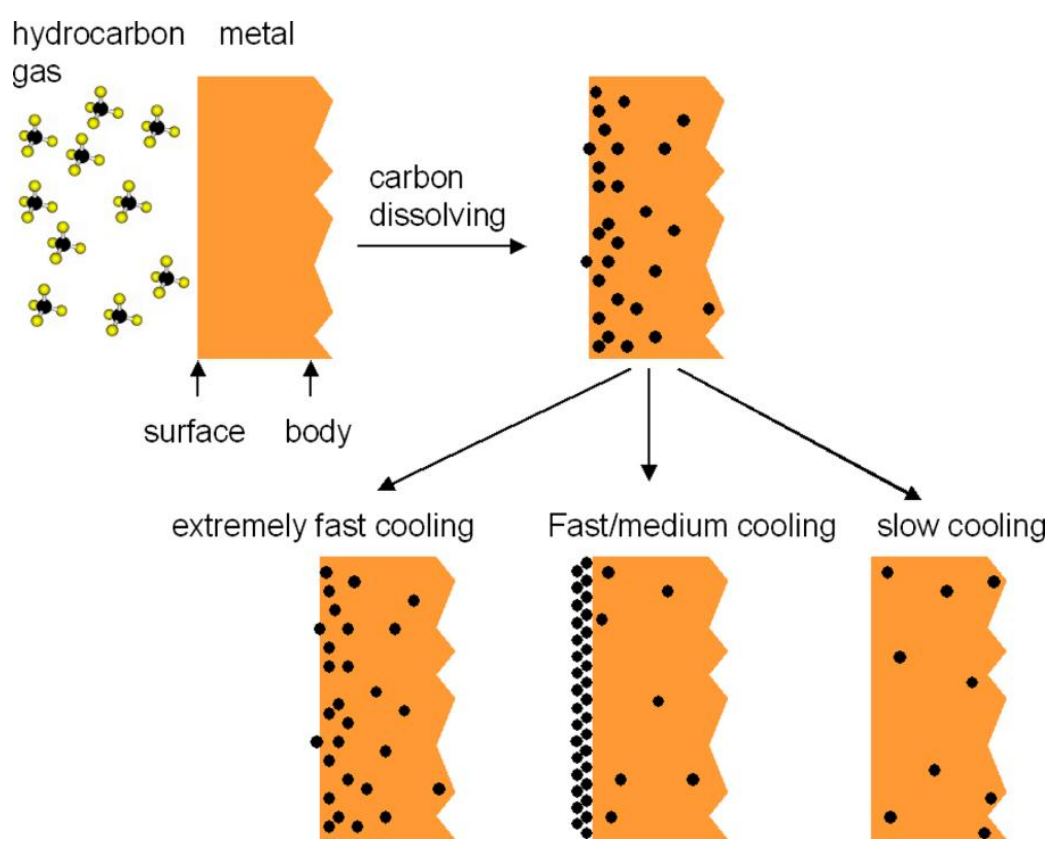

Figure: 2.5. Illustration of carbon segregation at metal surface[85]

The first successful CVD synthesis of graphene was done using Ni as catalyst. This was the inspiration coming from the CNT growth where Ni is a well-known efficient catalyst. Reduction of the partial pressure of the precursor is the main difference between graphene and CNT growth. This was done due to the low growth rates needed for the growth of graphene which is a sub-nanometer film. High growth rate leads to over deposition and disruption of the graphene structure. Graphene can also be fabricated via atmospheric pressure CVD, in which case a huge amount of argon gas is usually used to purge of the residue oxygen in the chamber to protect the metal surface from being oxidized, beside hydrogen gas, and to dilute the precursor to lower deposition rate.

Both CVD growth of Graphene and CNTs are not based on solid understanding of the mechanism. When the precursor decomposes into carbon ad-atoms on the Ni surface, they soon dissolve into the bulk Ni. This is due to the finite solubility of carbon in $\mathrm{Ni}$ at high temperatures $\left(\sim 900{ }^{\circ} \mathrm{C}\right)$. During cooling, solubility of carbon in Ni will 
reduce. Solubility of carbon in Ni is temperature-dependent, and hence the excessive dissolved carbon atoms have no way out but to precipitate back onto the top surface, where they form graphene sheet. Carbon solubility difference in $\mathrm{Ni}$ at the deposition temperature and the rate of cooling at the last growth step are the key mechanism that determines the thickness of graphene. Once the carbon atoms are precipitated, they do not arrange themselves uniformly on the Ni surface. Instead they precipitate onto the sites with high surface energy such as rough areas and grain boundaries. That is the reason for the non-uniformity of the graphene sheets deposited on nickel. The early growths of graphene deposited via CVD was conducted using $\mathrm{Ni}$ as catalyst. The biggest challenge in graphene growth on $\mathrm{Ni}$ raises from the pyrolysis of the precursor species. Hydrocarbon species are typically used as precursors for graphene growth, such as methane $\left(\mathrm{CH}_{4}\right)$, acetylene $\left(\mathrm{C}_{2} \mathrm{H}_{2}\right) . \mathrm{CH}_{4}$ is one of the most commonly used precursors, as it is comparatively stable at high temperature around $1000{ }^{\circ} \mathrm{C}$. Another challenge comes from the unique dimension of graphene. Graphene is a subnanometer-thick material. The typical CVD techniques use to grow more thick films. The deposition technique of graphene should essentially provide extremely precise control of the thickness of the deposited film analogous to the atomic layer deposition (ALD) with the deposition resolution of a single atomic layer.

\subsection{Synthesis of Graphene by chemical vapor deposition}

Figure 2.6 shows schematic diagram of the CVD system. CVD system is composed a gas delivery system which consist of gas cylinders for gas feed, mass flow controllers (MFC) to control the flow rates of gases and high temperature tube furnace finally the pressure control system and gas removal system to remove the byproducts during the graphene growth. 


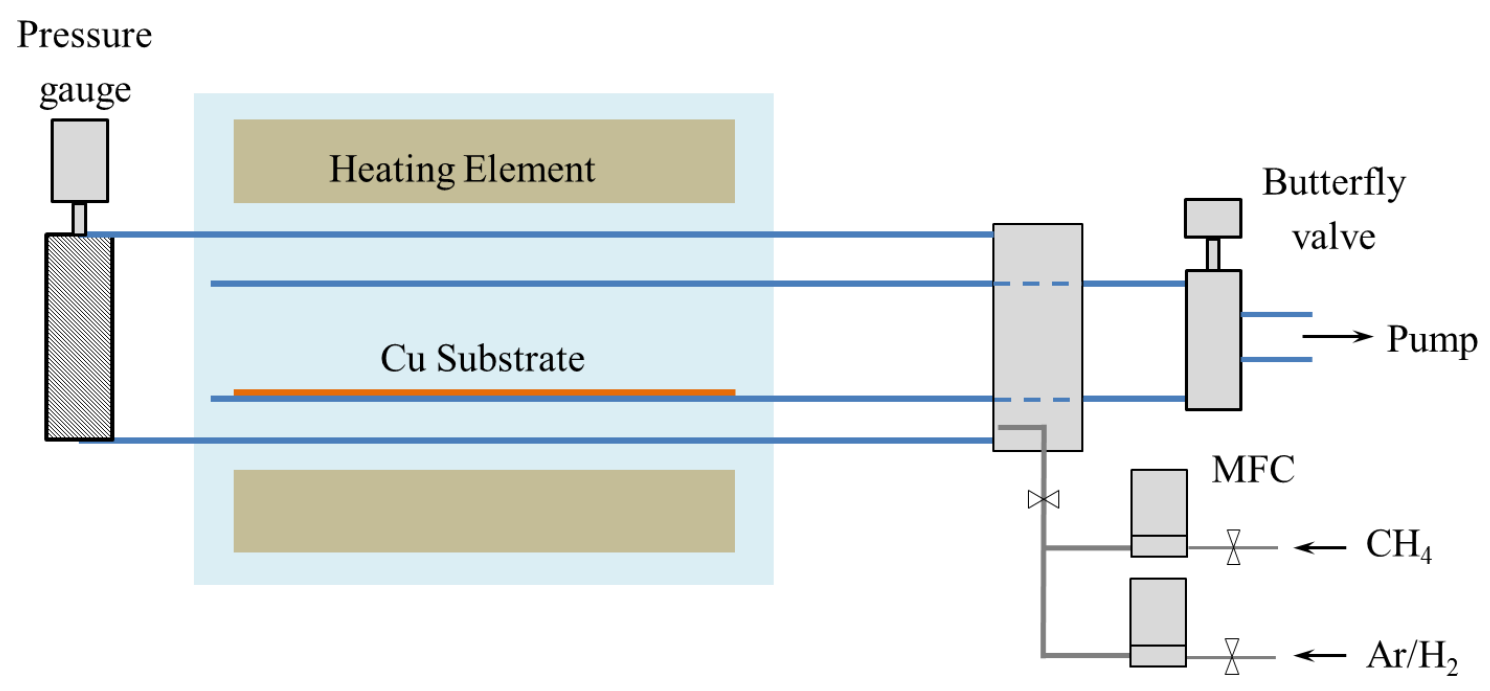

Figure 2.6: Schematic diagram of the CVD system for graphene synthesis

Graphene is grown on copper foil. First, the substrate are cleaned with acetone and isopropanol. Then the rolled foil is pushed in to the inner tube to reach the largest lateral dimension of the copper. Copper sits inside the furnace area. This dual tube design helps to maintain the undisturbed temperature profile along reactor area. This is achieved by preheating the reactor gas before it enters in the reactor area. Scalable synthesis of graphene is easily achievable in our system. 


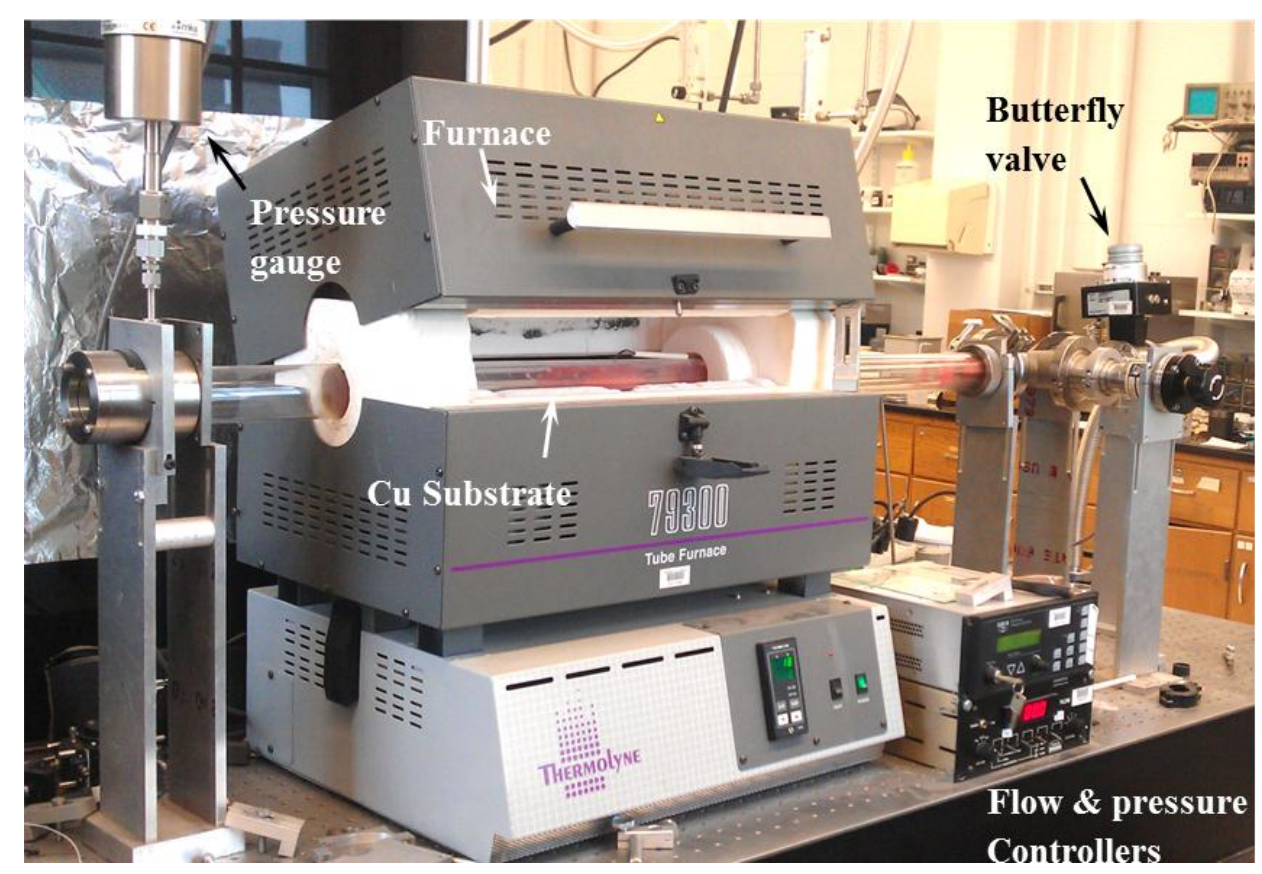

Figure 2.7: CVD system for graphene synthesis

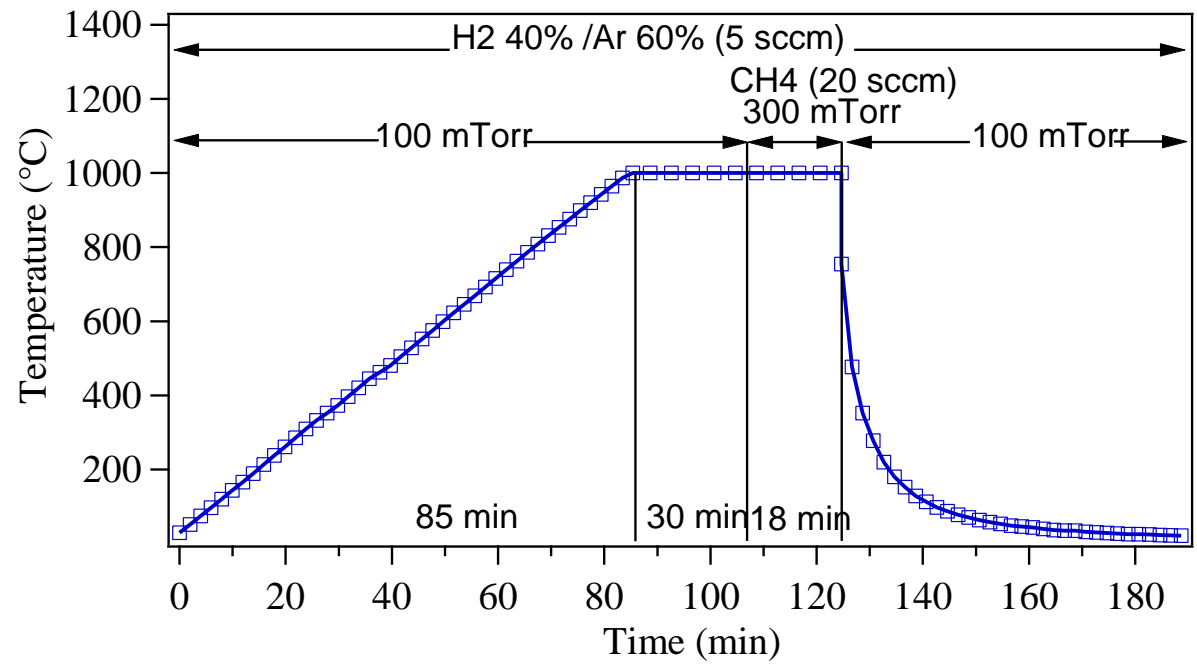

Figure 2.8: Graphene growth process 
First, the system is pumped to a base pressure of $~ 20$ mTorr with the butterfly valve fully open. Argon and Hydrogen mixture $\left(\mathrm{Ar} 60 \%, \mathrm{H}_{2} 40 \%\right)$ is introduced at a flow

rate of $5 \mathrm{sccm}$ to the system to start with the graphene synthesis process. This will drive away any air from the reactor chamber to minimize any contamination. Pressure controller is set to $100 \mathrm{~m}$ Torr. This will control the butterfly valve to achieve the preset pressure value. Temperature of the furnace is ramped from room temperature to $1000{ }^{\circ} \mathrm{C}$ at a rate of $10{ }^{\circ} \mathrm{C} / \mathrm{min}$. Once the temperature reaches the set point, sample is annealed for 30min to minimize any wrinkles or grain boundaries on the copper foil. Methane is introduced at a rate of $20 \mathrm{sccm}$ to the system as the carbon feed stock for graphene synthesis. The synthesis time is maintained $\sim 30$ minutes at 300 mTorr pressure and then methane is turned off. Finally $\mathrm{Ar} / \mathrm{H}_{2}$ is introduced to the system while the temperature of furnace is brought down to room temperature. The pressure control system controls the pressure from the beginning to the end of the synthesis process. The mechanical pump is used to pump out all the exhaust produced during the synthesis process. Complete graphene growth process is shown in Figure 2.8.

\subsection{Chemical Vapor Deposition of Graphene: Characterization}

\subsubsection{Scanning electron microscope}

The Scanning electron microscope (SEM) has main two components. They are electronic console and the electron column. The electronic console allows controlling the components such as filament current, accelerating voltage, focus, magnification, brightness and contrast etc. The electron column direct the beam of electrons to the sample for imaging.

The electron column is where the electron beam is generated under ultra-high vacuum and this beam is then focused into a small diameter spot. Beam is scanned across the 
surface of a specimen by electromagnetic deflection coils. Sample is placed in the chamber at the bottom of the column. The secondary electron detector is located above the sample stage inside the specimen chamber. Specimens are mounted and secured onto the stage which is controlled by a goniometer. Figure 2.9 shows a diagram of the electron column and a description of each of the components of the electron column[86].

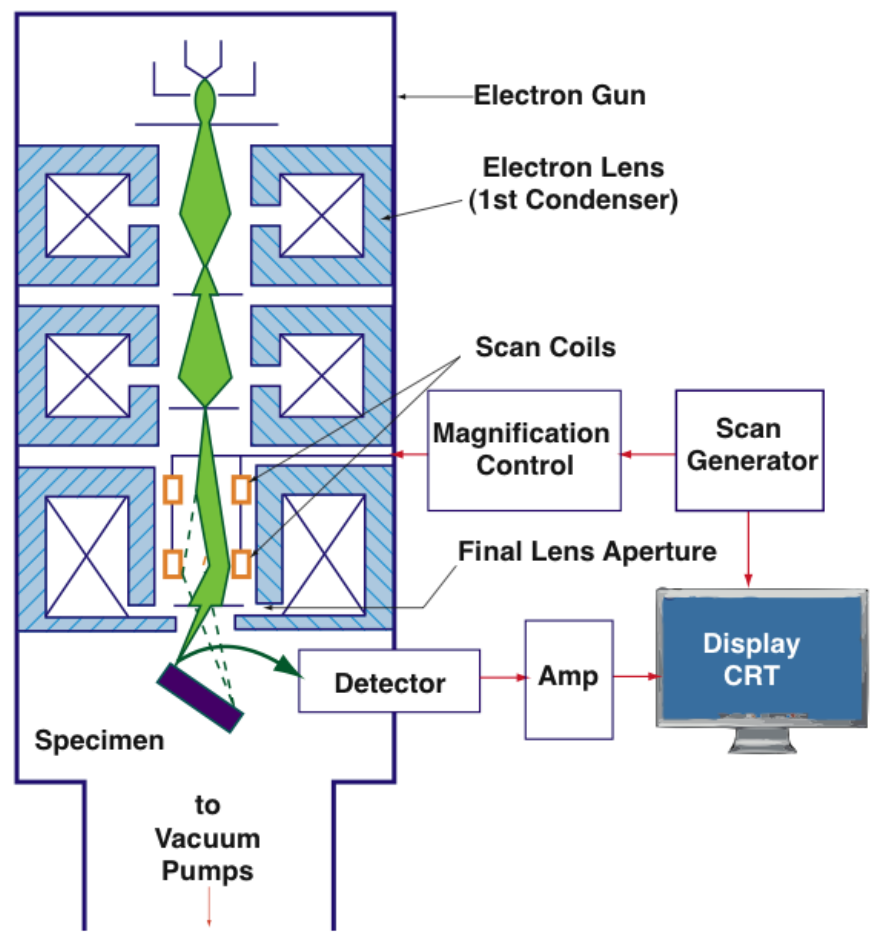

Figure 2.9. Scanning electron microscope column[86].

Electron gun is located at the top of the column where free electrons are generated by thermionic emission from a tungsten filament at $\sim 2700 \mathrm{~K}$. Electrons are primarily accelerated toward an anode that is adjustable from $200 \mathrm{~V}$ to $30 \mathrm{kV}(1 \mathrm{kV}=1000 \mathrm{~V})$. When electron beam passes the anode it is influenced by two condenser lenses that cause the beam to converge and pass through a focal point. This will focus the electron beam down to 1000 times its original size. Apertures in the beam path will reduce and exclude extraneous electrons in the lenses. The final lens aperture located 
below the scanning coils determines the diameter or spot size of the beam at the specimen. The spot size on the specimen will in part determine the resolution and depth of field. Decreasing the spot size will allow for an increase in resolution and depth of field with a loss of brightness. Images are formed by scanning the electron beam across the specimen using deflection coils inside the objective lens. The stigmator that located in the objective lens uses a magnetic field to reduce aberrations of the electron beam. This electron beam should have a circular cross section when it strikes the specimen[86, 87].

SEM Colum should be in ultra-high vacuum to provide a controlled electron beam. This vacuum is at a pressure of at least $5 \times 10^{-5}$ Torr. There are several reasons for having such high vacuum. The current that passes through the filament causes the filament to reach temperatures around 2700K[88]. Vacuum will eliminate any oxidation of the tungsten filament. Dust-free environment will provide the ability of column optics to operate properly. Air particles and dust inside the column can interfere and block the electrons before the ever reach the specimen in the sample chamber. In order to provide adequate vacuum pressure inside the column, a vacuum system consisting of two or more pumps is typically present[86].

\section{Interaction Volume}

Interaction volume of the primary beam electrons and the sampling volume of the emitted secondary radiation are two important concepts of SEM images and in the proper application of quantitative x-ray microanalysis. The image details and resolution in the SEM are determined by the size and characteristics of the interaction volume. 


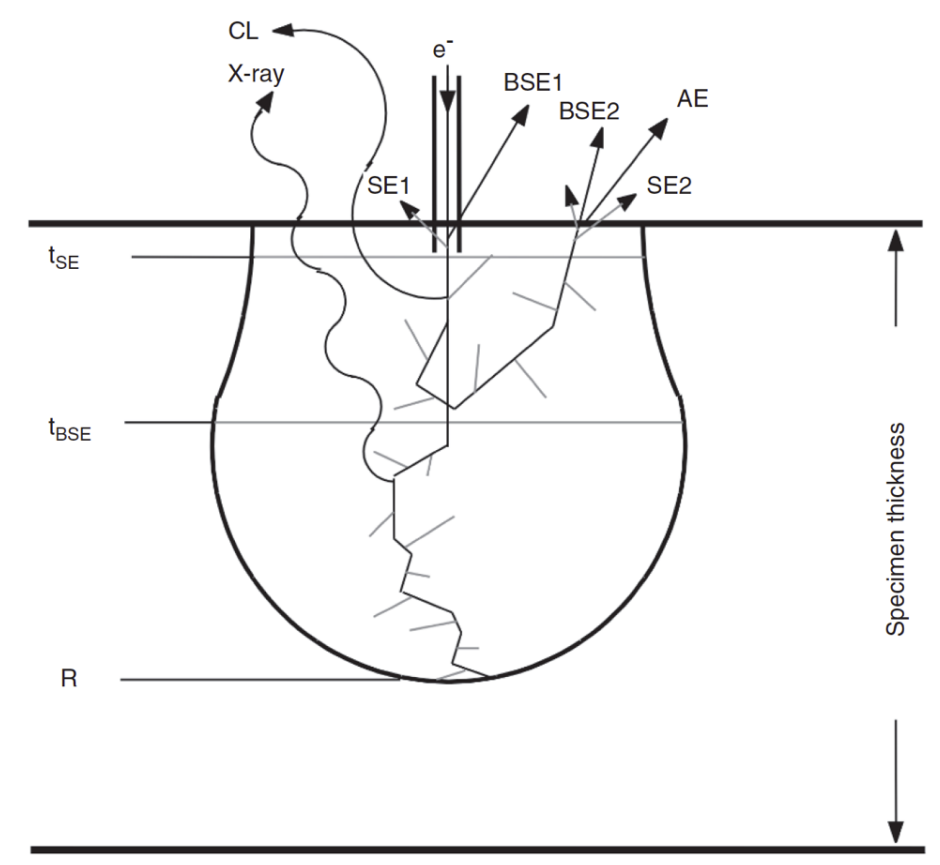

Figure 2.10: beam-specimen interaction

When the accelerated beam of electrons strikes a specimen they penetrate inside it to depths of about $1 \mu \mathrm{m}$. This beam interact both elastically and inelastically with the solid. This limiting interaction volume causes emergence of various types of radiation, including back scattered electrons, secondary electrons, characteristic and brehmsstrahlung x-rays, and cathodoluminescence in some materials.

Elastic and inelastic scattering controls the penetration of the electron beam into the solid. The resulting region over which the incident electrons interact with the sample is known as interaction volume. The interaction volume determines the nature of imaging in the SEM. The energy deposition rate varies rapidly throughout the interaction volume. The interaction volume for low atomic number target has a distinct pear shape as in Figure 2.10 and hemi-sphere shape for intermediate and high atomic number materials. The interaction volume increases with increasing incident beam energy and decreases with increasing average atomic number of the specimen. Secondary electrons originate form sampling depths from 10 to $100 \mathrm{~nm}$ and diameter 
equals the diameter of the area emitting backscattered electrons. Back scattered electrons are emitted from much larger depths compared to secondary electron. Ultimately the resolution in the SEM is controlled by the size of the interaction volume.

Image formation

The SEM image is a 2D intensity map in the analog or digital domain. Each pixel of computer video memory corresponds to a point on the sample. The display intensity is proportional to the signal intensity captured by the detector at each specific point. Unlike in optical or transmission electron microscopes no true image exists in the SEM. It is not possible to place a film anywhere in the SEM and record an image. The image is generated and displayed electronically. The images in the SEM are formed by electronic synthesis, no optical transformation takes place, and no real or virtual optical images are produced in the SEM.

Secondary electrons detector

This is the most common imaging mode. This detector collects the low-energy $(<50 \mathrm{eV})$ secondary electrons that are ejected from the k-orbitals of the specimen atoms by inelastic scattering interactions with beam electrons. These electrons are low energetic and originating within a few nanometers from the sample surface[89]. These secondary electrons are detected by an Everhart-Thornley detector[90]. This detector is a type of scintillator-photomultiplier system. The amplified electrical signal from the photomultiplier tube is displayed as a two-dimensional intensity distribution that can be viewed and photographed on an analogue video display. This signal is subjected to analog-to-digital conversion and displayed. This process relies on a raster-scanned primary beam. The brightness of the signal depends on the number of secondary electrons reaching the detector. If the incident electron beam is 
perpendicular to the surface, then the activated region is uniform about the axis of the beam. If the beam incident by an angle, the "escape" distance of one side of the beam will decrease, so more secondary electrons will be emitted. So, steep surfaces and edges will be brighter than flat surfaces. This will result three dimensional appearances in the images.

\section{Backscattered electron detector}

Backscattered electrons are high energetic electrons that originating from original electron beam by elastically interacting with specimen atoms. High atomic number elements will have strong backscatter electrons. This will create brighter images. Backscatter electrons are used to detect contrast between areas with different chemical compositions[89]. Dedicated backscattered electron detectors are positioned above the sample in electron column. This detector is concentric with the electron beam and will maximize the solid angle of collection. There are two main types of backscatter electron detectors. They are scintillator and semiconductor types. When all parts of the detector are used to collect electrons symmetrically about the beam, atomic number contrast is produced. However, strong topographic contrast is produced by collecting back-scattered electrons from one side above the specimen using an asymmetrical, directional BSE detector; the resulting contrast appears as illumination of the topography from that side. Semiconductor detectors can be made in radial segments that can be switched in or out to control the type of contrast produced and its directionality[91].

\subsubsection{Raman Spectroscopy}

Single layer graphene is the simplest crystal structure in the family of $\mathrm{sp}^{2}$ carbon systems. It has the highest symmetry and the simplest Raman spectra (see Figure 
2.11). After the isolation of graphene in 2004, large research communities that had the knowledge about the Raman spectroscopy associated with other carbon structures started using Raman for characterization for graphene[92-94]. For example, the detailed study of effects of inter-layer coupling on the electronic structure was carried out using the dispersion of the $2 \mathrm{D}$ band when changing the excitation laser energy in bi-layer graphene[95]. Strain [96-98], charge transfer and disorder effects due to doping, top gates and different types of substrates were addressed using the G and 2D bands of graphene $[95,96,98-101]$. One layer graphene is an ideal system to study defects using Raman technique, because there are no aspects related to the penetration depth [102-104]. Raman studies in graphene are helping our basic understanding of long standing experimental results on carbon nanotubes and other carbon based systems.

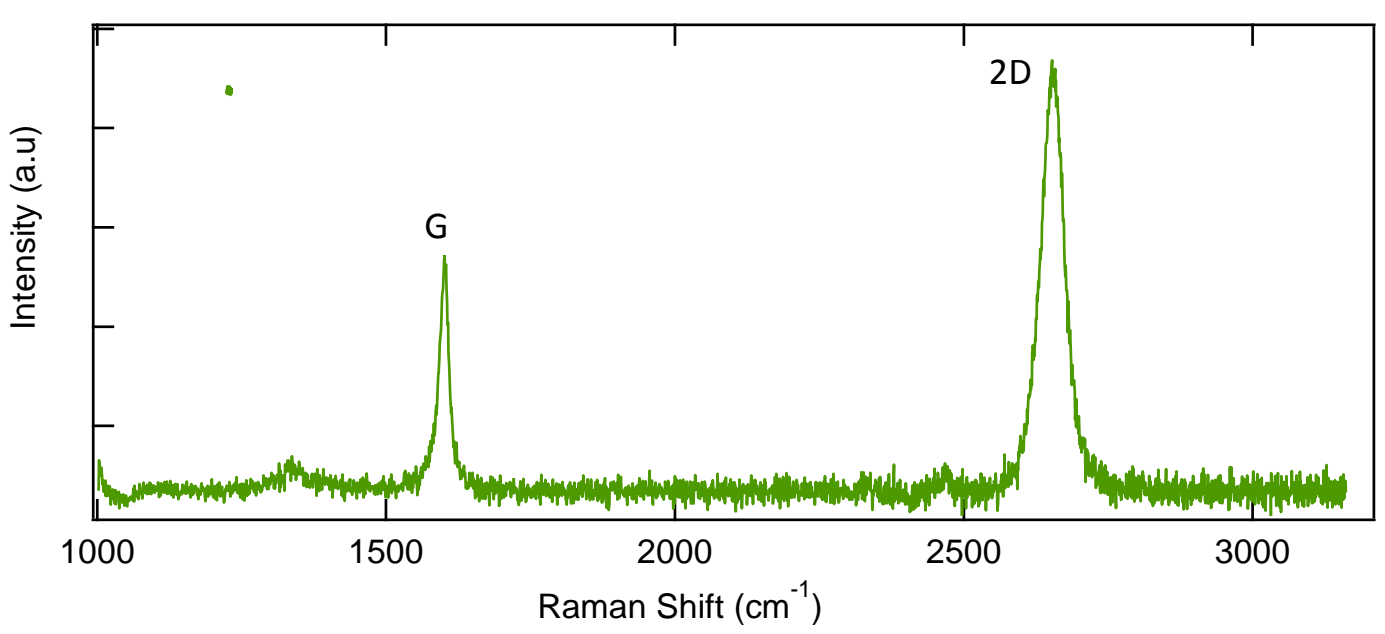

Figure 2.11: Raman spectrum of single layer graphene on $\mathrm{SiO}_{2}$ by $632 \mathrm{~nm}$ excitation laser. The two most intense features are named the $\mathrm{G}$ and 2D peaks. The Raman spectrum of pristine mono-layer graphene is unique among $\mathrm{sp}^{2}$ carbons.

\section{The G band}

The $\mathrm{G}$ band is a very important spectral feature of graphene and derived from in plane motion of the carbon atoms and appears near $1580 \mathrm{~cm}^{-1}$ as shown in Figure 2.11. This 
peak is extremely sensitive to strain effects and is also a good indicator of the number of graphene layers. $\mathrm{G}$ band position tends to shift as the number of layers increases. This shifts towards the lower frequencies and follows a $1 / n$ dependence on the number of layers $n$ [105]. There is no significant change in spectral shape. $G$ band is also sensitive to doping and both the frequency and the line width of this peak can be used to monitor the doping level[106].

\section{The D band}

$\mathrm{D}$ band is also known as disorder band for historical reasons. This peak is due to lattice motion away from the center of the Brillouin zone and it appears between 1270 $\mathrm{cm}^{-1}$ and $1450 \mathrm{~cm}^{-1}$. Exact position will depends on the excitation wavelength[107]. The D band indicates presence of defects or edges in the graphene sample. In fact, a definitive explanation of its origin and dependence on excitation wavelength is derived from the double resonance theory originally proposed by Thomsen[108]. In theory, intraband phonon scattering of the electron requires momentum that is easily taken up by defects, which is why this band was first observed in defective crystals.

\section{The 2D band}

The $2 \mathrm{D}$ band, also called as $\mathrm{G}^{\prime}$ is a result of a second order two phonon process. It exhibits an unusually strong frequency dependence on the excitation laser due to a double resonance process. This will link the phonon wave vector to the electronic band structure[107].

This peak appears approximately at $2700 \mathrm{~cm}^{-1}$ for a $632 \mathrm{~nm}$ laser excitation. This peak is also used for determination of the number of graphene layers. However this peak has a more complex behavior than the frequency shift observed for the $G$ band. The 
2D peak of the monolayer graphene can be fitted with a single Lorentzian peak while bilayer graphene requires four Lorentzian peaks. This is related to the four possible double resonance scattering processes. Only one is possible for the monolayer. The number of double resonance processes also increases with the number of layers. This will eventually converge to the spectral shape to graphite. Only two peaks are observed in graphite [109] in general.

\section{Layer number dependence of $2 \mathrm{D}$ band}

Raman 2D band has been used to characterize the number of layers in few layer graphene samples. Not only number of layers, stacking order between these layers also can be found. Figure 2.12 shows the Raman spectra for highly ordered pyrolytic graphite (HOPG). 


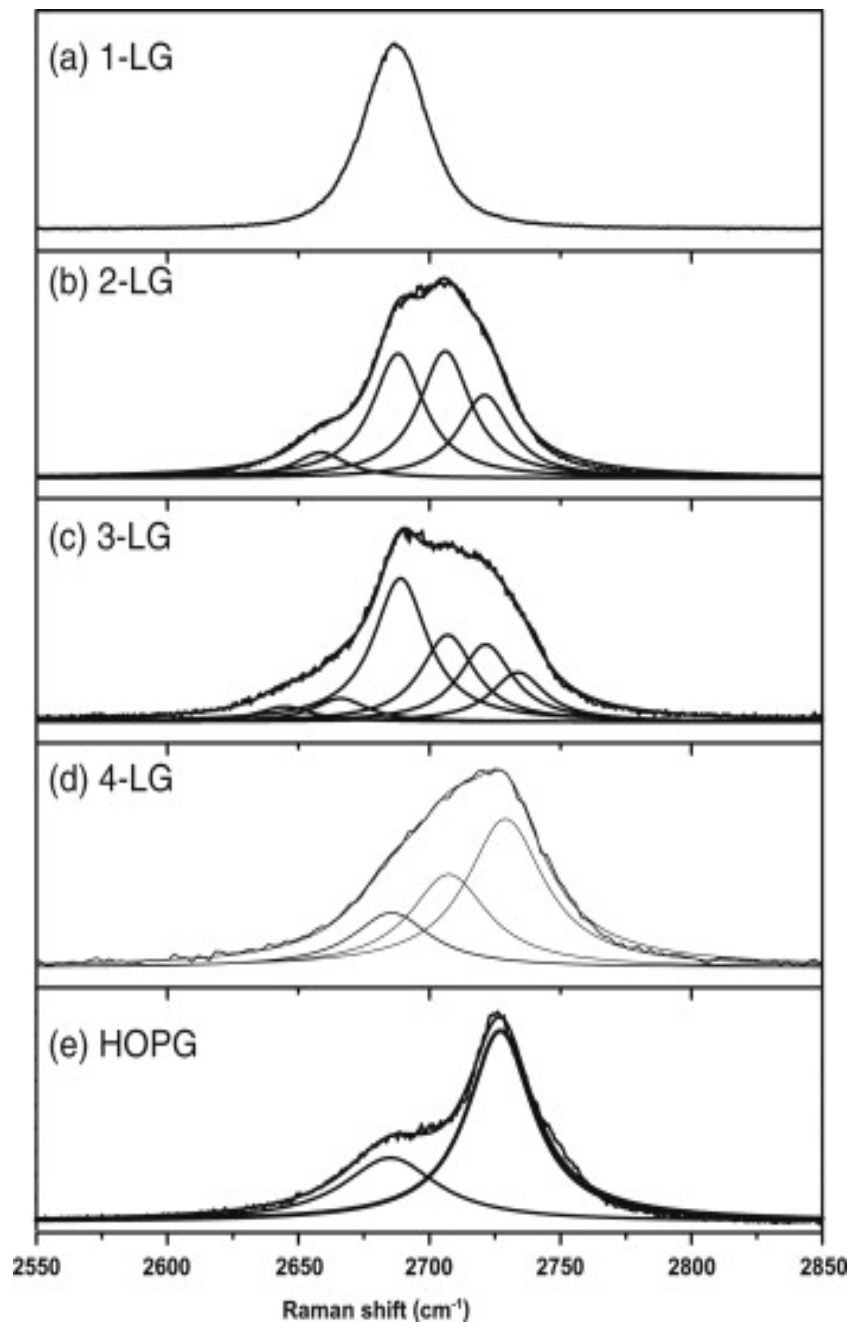

Figure 2.12: Raman 2D band for (a) 1-LG, (b) 2-LG, (c) 3-LG, (d) 4-LG and (e) HOPG[110].

Identification process of the number of layers by Raman spectroscopy is only for graphene samples that have $\mathrm{AB}$ Bernal stacking. Mechanically exfoliated graphene samples or HOPG graphite lead to graphene flakes that have predominantly $A B$ stacking. But for other growth techniques like turbostratic graphite has random stacking of the graphene layers. The absence of an interlayer interaction between the graphene planes makes the Raman spectra of turbostratic graphite look much like that for monolayer graphene. 


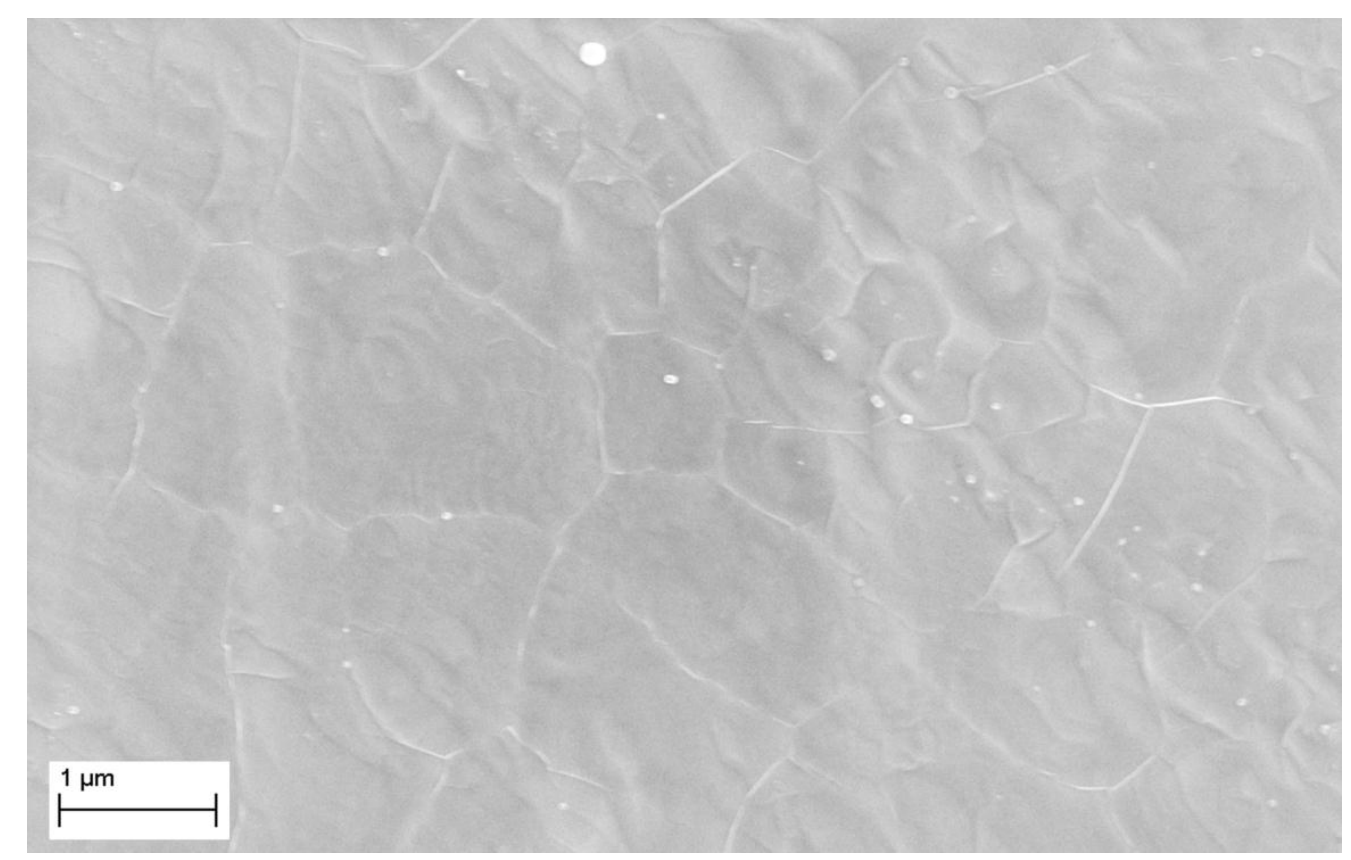

Figure 2.13: SEM image of graphene on a copper foil with a growth time of $18 \mathrm{~min}$

Figure 2.13 shows a scanning electron microscopy (SEM) image of graphene on a copper substrate where the $\mathrm{Cu}$ grains are clearly visible. This image of graphene on $\mathrm{Cu}$ shows the presence of $\mathrm{Cu}$ surface steps, graphene "wrinkles," and the presence of non-uniform dark flakes. These wrinkles associated with the thermal expansion coefficient difference between $\mathrm{Cu}$ and graphene are also found to cross $\mathrm{Cu}$ grain boundaries, indicating that the graphene film is continuous. 


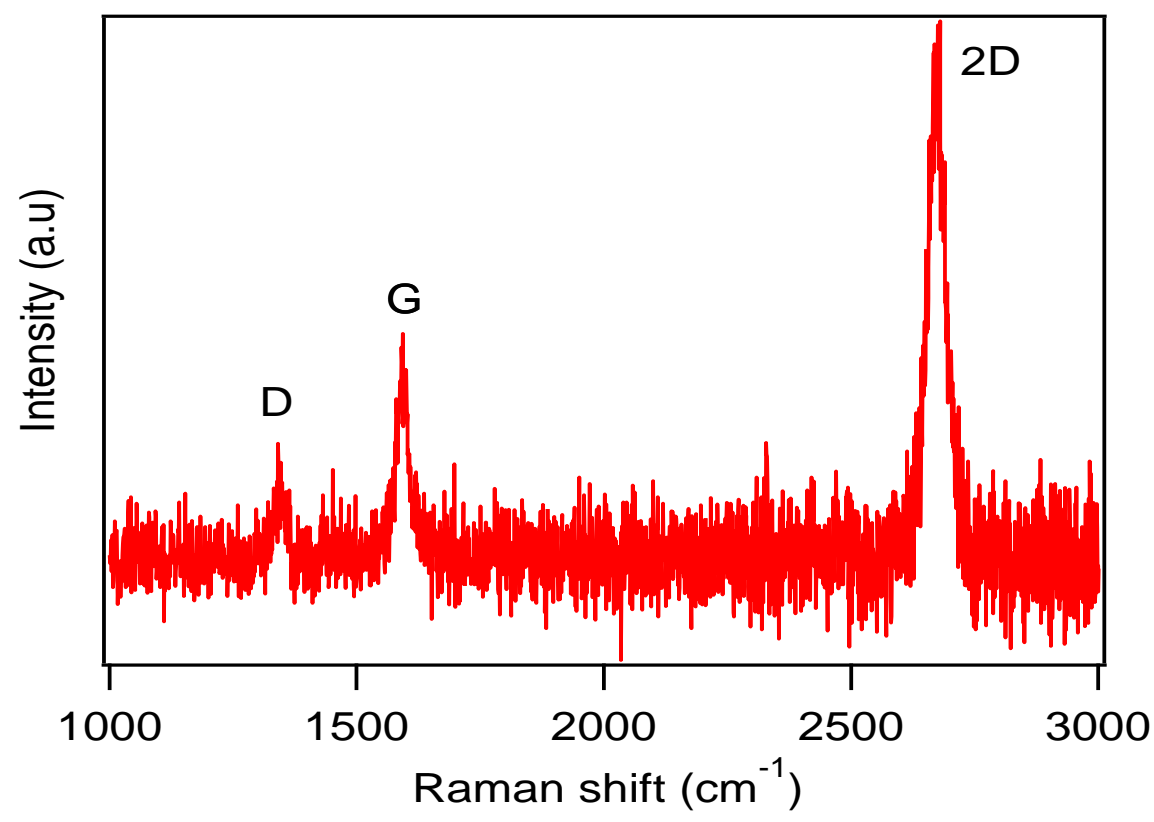

Figure 2.14: Raman spectra obtained from a graphene film grown on a thin copper film

The Raman spectra of graphene grown on thin copper film is shown in Figure 2.14. Raman D band intensity is an indication of degree of disorder in carbonaceous materials. G-peak intensity of graphene on copper is an indication of uniformity, except in regions corresponding to wrinkles or graphene grain boundaries. Growth time and cooling rate does not affect the graphene thickness on copper. Furthermore the deposition of a continuous graphene layer leads to the passivation of the $\mathrm{Cu}$ surface so that multi-layered growth is dramatically hindered[111].

\subsection{Graphene transfer process}

Any transport measurements done on graphene supported by copper will short the graphene through the substrate. So it is very crucial to remove graphene from the conducting catalyst surface and transferred onto an insulating surface. Not all the transfer techniques are applicable for CVD graphene. The most common graphene 
transfer techniques are involved with etching the underlying catalyst. During the etching process, graphene is supported by some kind of medium such as Poly(methyl methacrylate) (PMMA), Polydimethylsiloxane (PDMS) or thermal release tape. Then the polymer supported graphene can be scooped to a substrate.

Direct transfer technique consists of etching and direct scooping to the substrate. This is a straightforward transfer technique to move graphene from the surface of metals to the target substrate. Getting rid of the catalyst film underneath the graphene is the key idea. That allows the graphene sheet to be separated and scooped.

(a)

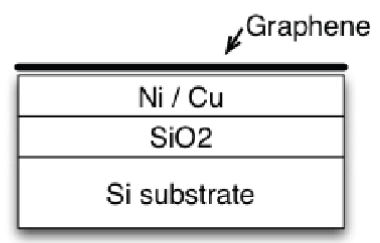

(c)

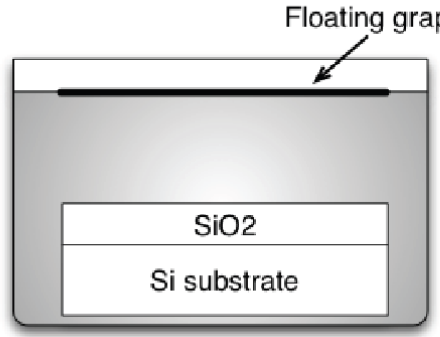

(e)

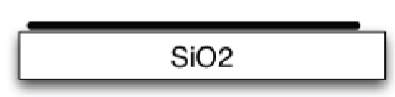

(b)

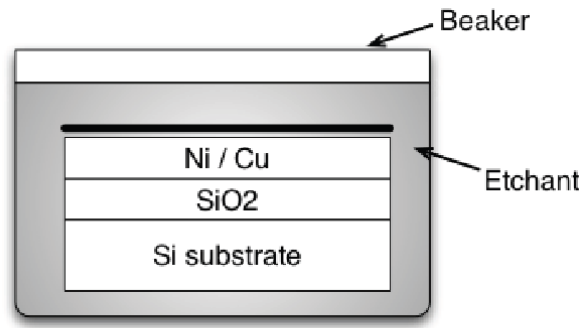

(d)

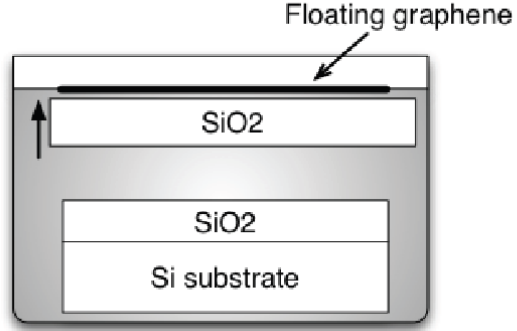

Figure 2.15: Schematic diagram of the etching and scooping method without using a support[112]

Direct transfer process is shown in Figure 2.15. After the CVD growth, whole sample is submerged in an etchantsolution to etch away the metal. Once the catalyst is completely etched away, the graphene sheet floats on the liquid surface due to its hydrophobic nature[113]. Scooping of the floating graphene on to a substrate must be done with extreme care. The advantage of this technique is that it is a simple process 
with few steps which reduces the possibility of introducing potential impurities to graphene. But the direct scooping method has low yield, because it requires high stability of the transferring setup to avoid the fragile graphene sheet from breaking during the process. Even the etchant solution can flow between the transferred graphene and its target substrate. This can introduce chemical dopants and enhances carrier scattering to degrade the electrical properties of graphene

(a)

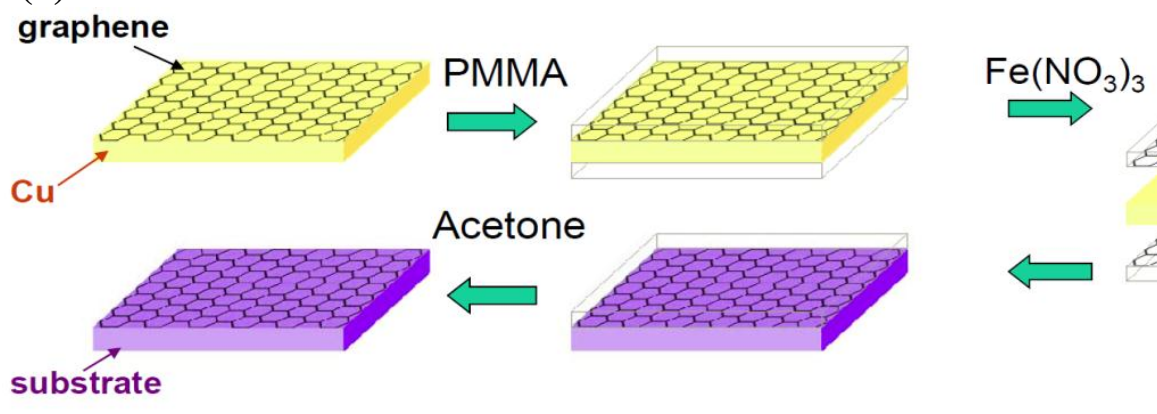

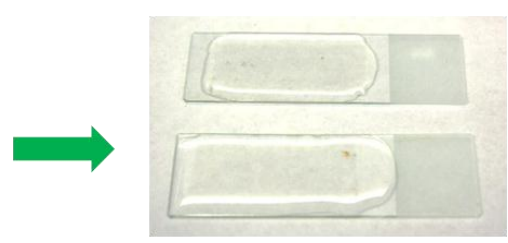

PMMA removal

(b)

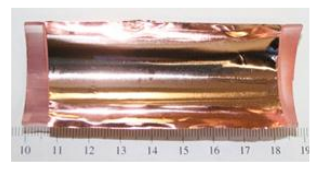

Graphene on copper

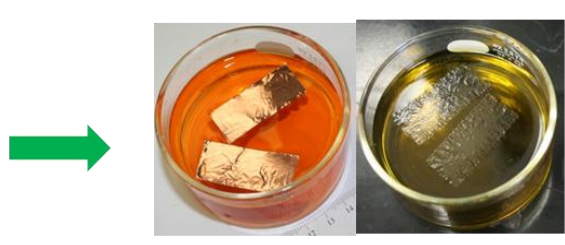

PMMA supported graphene

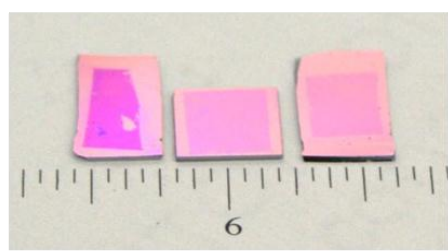

Graphene on $\mathrm{SiO}_{2} / \mathrm{Si}$ substrate

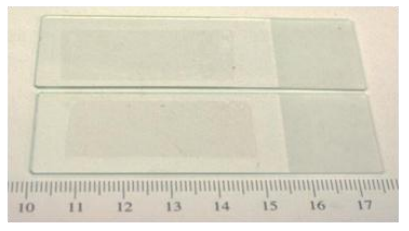

Graphene on glass substrate

Figure 2.16: Graphene transfer with PMMA as a support (a) Schematic diagram for the transfer process (b) pictures of the process.

On the other hand transfer of graphene using a polymer medium can increase the yield. This mechanism is similar to the previous but the graphene will be supported by a PMMA layer during the transfer. Figure 2.16a shows a schematic diagram of the 
transfer process. There are other commonly used support materials, such as PDMS and thermal release tape. Both PDMA and thermal tape are not dissolved at the last step. Instead they are peeled at the final stage of the process. It is critical that the interface bonding between graphene and substrate is greater than that between PDMS and graphene to ensure graphene is left on substrate while being peeled off.

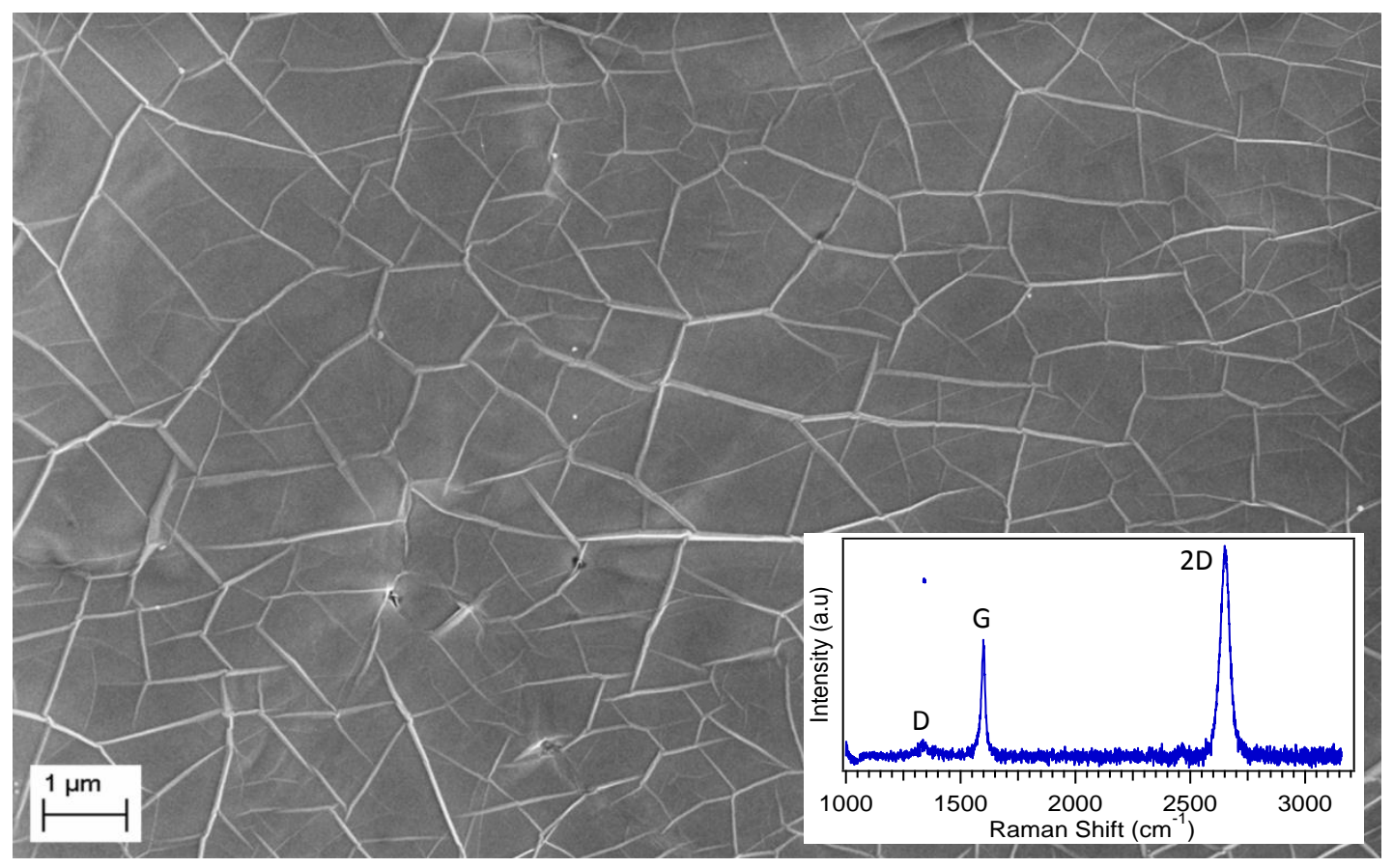

Figure 2.17: SEM image of graphene transferred on to $\mathrm{SiO}_{2} / \mathrm{Si}$ substrate

Figure 2.17 shows the SEM image of a graphene transferred on to $\mathrm{SiO}_{2} / \mathrm{Si}$ substrate by polymer supported technique. The overall observation shows that the graphene film is more or less homogeneous on whole substrate surface. At micrometer resolution the main topology features of the film are the flat domains with characteristic size of about $1 \mu \mathrm{m}$ and the wrinkles between the domains. The main reason for the origin of the wrinkles is the post grown deformation of the graphene films due to the difference in the thermal expansion coefficients of graphene and copper[114, 115]. These surfaces consist of highly ordered few layer graphene films with thickness varying between one to tens graphene layers. 


\section{CHAPTER 03}

\section{HYDROGEN FUNCTIONALIZATION OF GRAPHENE}

\subsection{Introduction}

Graphene has impacted numerous fields in science due to its remarkable features such as magneto transport, electromechanical modulation, extremely high carrier mobility, tunable band gap, quantum Hall effect, Klein tunneling nature, and electron confinement effects[116, 117] etc. These properties makes Graphene suitable for use in field-effect transistors, transparent conducting films, lithium ion batteries, super capacitors and ultra-sensitive sensors[118, 119] etc. Functionalization is one of the major methods of changing physical and mechanical properties of Graphene[120, 121]. Functionalization is mainly divided in to two categories, namely covalent or non-covalent. Noncovalent functionalization is based on intermolecular forces between different functional groups to Graphene. Noncovalent functionalization is more favorable than covalent due to the integrated structure and excellent performance of Graphene[122]. 


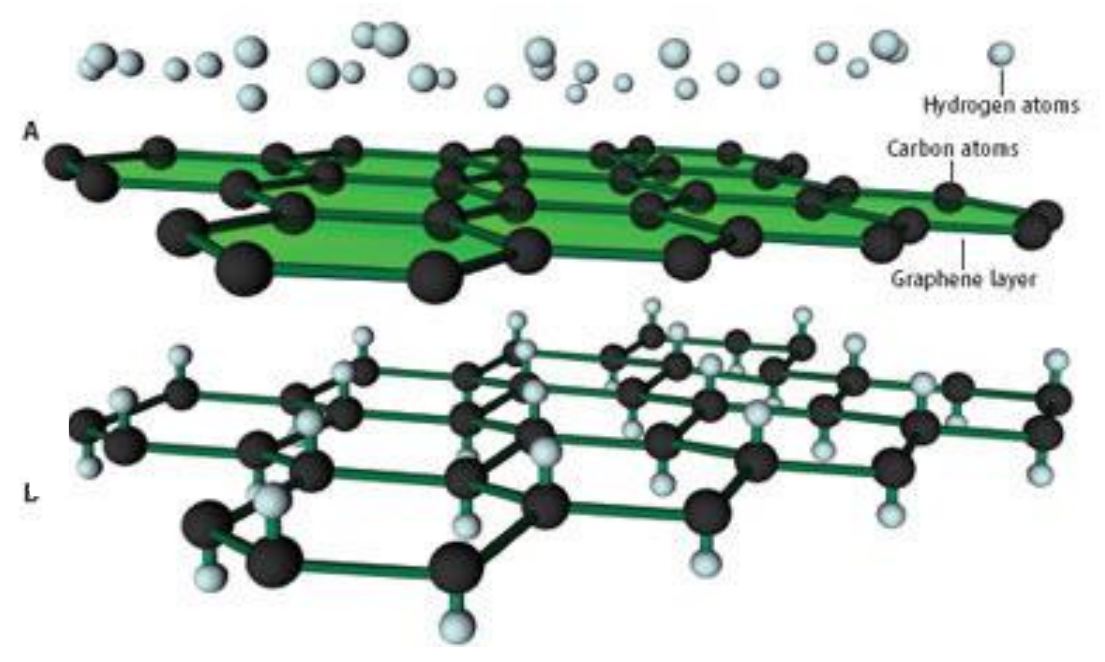

Figure 3.1: Hydrogenation of Graphene[123]

Chemical elements such as oxygen, hydrogen or fluorine when adsorbed on the surface of Graphene, yield covalent functionalization which results in much stronger modification of the geometric and electronic structures of Graphene[124-126]. Most covalent methods introduce structural damage and residual deposition on Graphene which affects the performance of the device. In Graphene oxide, oxygen species are randomly attached to the Graphene lattice by breaking the $\mathrm{C}-\mathrm{C} \pi$ - and $\sigma$-bonds which results in a breakdown of conductivity[127]. In contrast hydrogenation offers a more controlled means of attaching hydrogen species to the $\mathrm{sp}^{2}$ carbon atoms at least on one side of Graphene by breaking the $\mathrm{C}-\mathrm{C} \pi$ bonds. This allows the preservation of the crystalline order of the carbon lattice, but leads to re-hybridization of the carbon atoms from $\mathrm{asp}^{2}$ to a distorted $\mathrm{sp}^{3}$ state, which is expected to induce the formation of a band gap[128]. In previous studies, the electrical transport, optical, and structural properties have been studied on hydrogenated Graphene-based-FETs by altering the carrier density of randomly hydrogenated Graphene by changing the gate voltage[128-131]. There have been some studies to alter the transport properties of Graphene by subjecting it to hydrogen plasma at varying temperatures[132] and 
residence time[133]. Here, for the first time we report on well controlled and sequential hydrogenation of Graphene causing it to undergo the metal-insulator transition.

The fractional Quantum-Hall transitions, magnetic transitions of cuprates and heavy-Fermion alloys, metal-insulator and superconductor-insulator transitions in disordered alloys are all well-known quantum phase transitions[134]. These transitions are generally complicated by the strong electron-electron interactions and the presence of static disorders[135]. Covalent functionalization of Graphene offers a platform to test such transitions. This chapter reports a series of experiments that describe the dependence of the Four-probe resistance $(R)$, Thermopower $(S)$, Hall mobility $(\mu)$, and the Magneto resistance $(M R)$ on the temperature $(T)$ and the degree of hydrogenation for sequentially hydrogenated Graphene through the metal-insulator transition. These transport properties have been well supported by changes in the structural properties characterized with Raman spectroscopy and X-ray photoemission spectroscopy. We have observed different localization regimes that have not been observed before.

\subsection{Transport measurements on graphene}

Transport properties were measured by anchoring two miniature thermocouples $($ Chromel $(\mathrm{KP}) / \mathrm{Au}-7$ at\%Fe $(\mathrm{Au}: \mathrm{Fe}))$ in $100 \mu \mathrm{m}$ diameter followed by two electrical concretions and a resistive heater on the sample as in Figure 3.2 
(a)

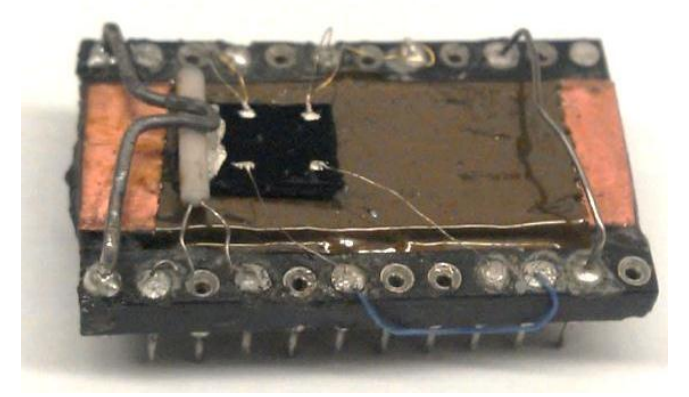

(b)

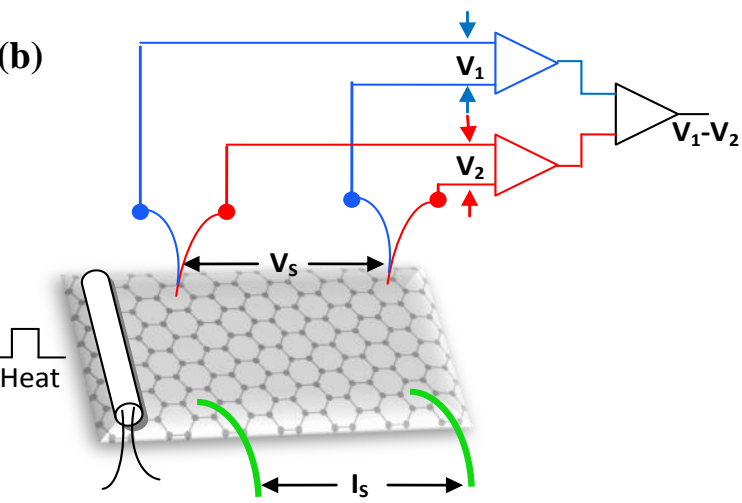

Figure 3.2: Electrical concretions on the graphene sample (a) picture (b) schematic diagram.

\subsubsection{Thermoelectric power of Graphene}

A temperature gradient in a conductor or a semiconductor will give rise to a built in electric field which will create a potential difference. This phenomenon is known as the thermoelectric effect or seebeck effect. Thermoelectric voltage per unit temperature difference is known as the Seebeck coefficient.
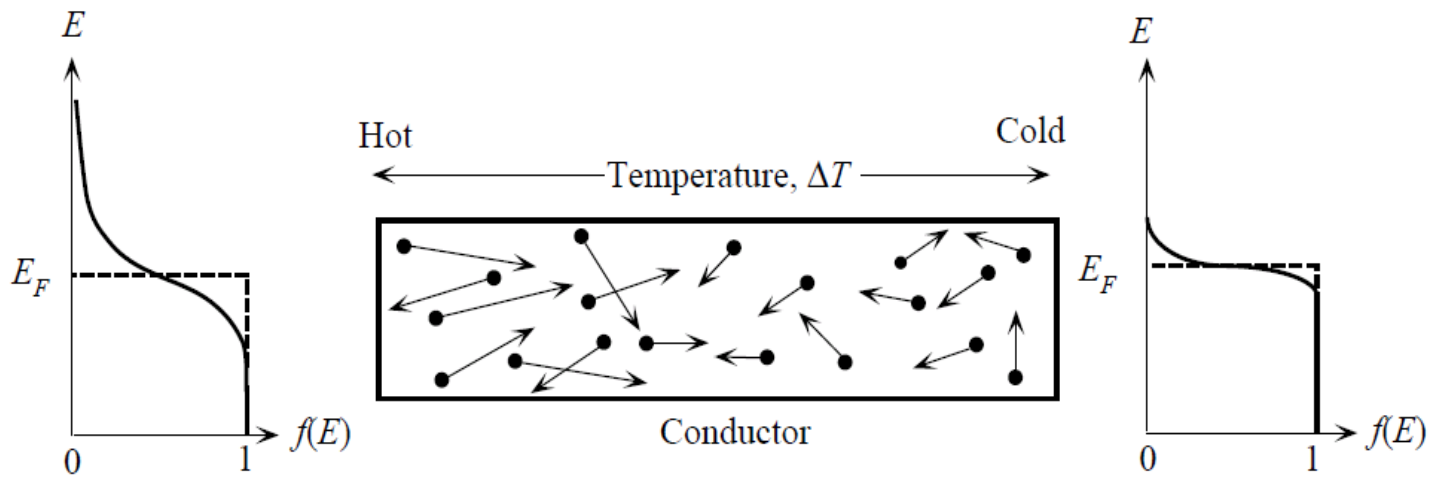

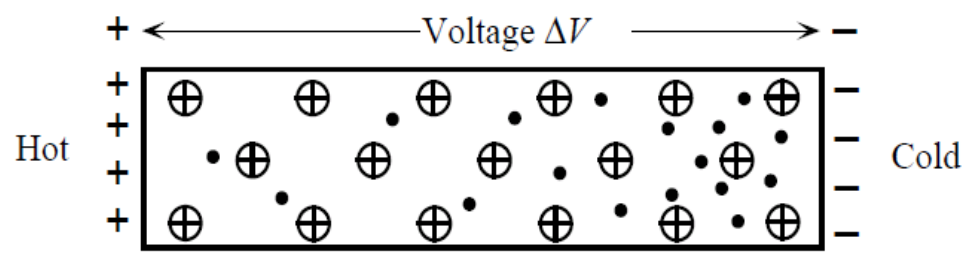

Figure 3.3: Built in potential due to the temperature gradient in metal [17].

Consider a metal sample that introduced heat from one end and other end kept cold as shown in figure 3.3. The electrons at the hot end are more energetic compared to the 
electron at the cold end. Therefore electrons at the hot end have greater velocities than those in the cold region. So there is a net diffusion of electrons from the hot end toward the cold end. This will leave behind exposed positive metal ions in the hot region and accumulates electrons in the cold region. This process continue until the positive ions at the hot region and the excess electrons at the cold region develops an electric field between to prevents further electron motion from the hot to cold end. This potential difference $\Delta V$ across the sample due to the temperature difference $\Delta T$ is called the Scebeck effect [17].

$$
S=\frac{\Delta V}{\Delta T}
$$

Sign of S depend of the majority carrier type of the sample. If electrons were the majority, carriers diffuse from hot to cold end. Then the cold side is negative with respect to the hot side and the Seebeck coefficient is negative. For a $\mathrm{p}$ type semiconductor holes are the majority carrier. The holes would diffuse from the hot to the cold end. The cold side would be positive with respect to the hot side which would make $S$ a positive quantity.

By considering the linear response approximation for the electrical current density and thermal current density, thermopower of graphene is given for low temperatures by [18],

$$
S=-\left.\frac{\pi^{2}}{3 e} \frac{T}{\sigma(\mu)} \frac{\partial \sigma(\varepsilon)}{\partial \varepsilon}\right|_{\varepsilon=\mu}
$$

The low temperature is defined as $\mathrm{T} \ll \mathrm{T}_{\mathrm{F}}$, where $\mathrm{T}_{\mathrm{F}}=\mathrm{E}_{\mathrm{F}} / \mathrm{k}_{\mathrm{B}}$ 
$\sigma(\varepsilon)$ is the energy dependent conductivity of graphene given by,

$$
\sigma(\varepsilon)=\frac{e^{2} v_{F}^{2} D(\varepsilon) \tau(\varepsilon)}{2}
$$

$v_{f}$ is the Fermi velocity and $D(\varepsilon)=2|\varepsilon| /\left(\pi h^{2} v_{F}^{2}\right)$ is the density of states. By considering the energy dependent scattering time to be $\tau \propto \varepsilon^{\mathrm{m}}$, thermopower at low temperatures can be written as,

$$
S=\frac{\pi^{2}}{3 e} \frac{k_{B} T}{T_{F}}(m+1)
$$

Here the exponent ' $m$ ' has weak temperature and the density dependence because $\tau$ behaves only as an effective power law in energy. sign of the thermopower cab change if $m<-1$. For high temperatures $\left(T \gg T_{F}\right)$ we can express the thermopower by,

$$
S \approx \frac{k_{B}}{e} \frac{(m+2)}{2} \frac{\left(e^{m+1}-1\right)}{\left(2^{m}-1\right)} \frac{\xi(m+2)}{\xi(m+1)}
$$

Figure 3.4 shows the calculated graphene thermopower for different scattering exponents $m$ as a of function of $T / T_{F}$. Dashed lines represents the Mott formula that agree with the full calculations for $T<0.2 T_{F}$. 


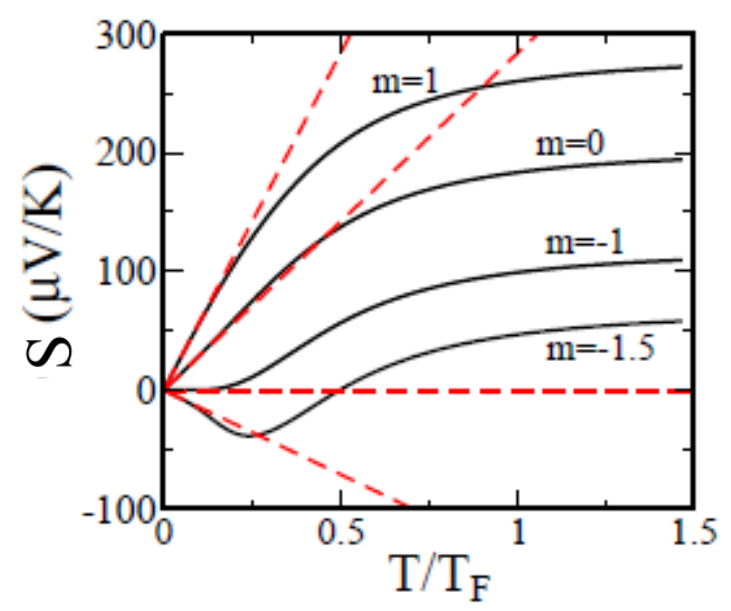

Figure 3.4: calculated graphene thermopower for different scattering exponents $m$.

Sign of the $\mathrm{S}$ expected to change when majority carriers of the sample change across the charge neutral point (Dirac point) as it changes from electrons to holes. Away from the charge neutral region the density dependence of $S$ behaves as $1 / \sqrt{ } n$. And $S$ exhibits linear temperature dependence in agreement with the semi classical Mott formula. S does not follow the $1 / \sqrt{ } n$ relation predicted by the Mott formula, Close to the Dirac point. This deviation is due to the quenched disorder induces strong density fluctuations that break up the density landscape in electron hole puddles [18].

By considering the simple two component model near the charge neutral regime, $\mathrm{S}$ can be written as,

$$
S=-\frac{k_{B}^{2}}{e} \frac{2 \pi^{2}}{3} \frac{T}{v_{F} \sqrt{\pi}}\left[\frac{\sqrt{n_{e}}-\sqrt{n_{h}}}{n_{e}+n_{h}}\right]
$$

Here, $n_{e}$ is the electron density and $n_{h}$ is the hole density. If there are equal number of electrons and holes, thermopower goes to zero. Overall sign is decided by the 
majority carriers. When the $\mathrm{S}$ is going through zero, conductivity of the sample goes through a maximum.

\subsubsection{Four Probe Resistivity - the van der Pauw Method}

The four probe resistivity measurement is a versatile method widely used to characterize materials. Electrical resistance of a test sample can be measured by attaching two wires to it, this inadvertently also measures the resistance of the contact point of the wires to the sample.

Typically the resistance of the point of contact called contact resistance is far smaller than the resistance of the sample. So contact resistance can be neglected. If very small sample resistance needs to measured, contact resistance may be dominant. Especially under variable temperature conditions, the contact resistance can dominate and completely change the temperature readout of the sample.

The effects of contact resistance can be eliminated by the use of van der Pauw Method. Figure 3.5, below shows a schematic of this method. In this schematic, four wires have been attached to the test sample to flow a constant current through the length of the sample. These leads are labeled as 1 and 2 in the Figure. Due to the resistance to the flow of electrical current through the sample, there will be a drop of voltage across the test sample. That voltage can be measured by using the leads 3 and 4. The sheet resistance of the sample is the ratio of the voltage across the probes 3 and 4 to the value of the output current though the probe 1and 2. Since there is no potential drop across the contact resistance associated with probes 3 and 4, only the resistance associated with the material between probes 3 and 4 is measured. Similarly, 
second measurement of resistance can be obtained by interchanging the current and voltage probes on the sample.

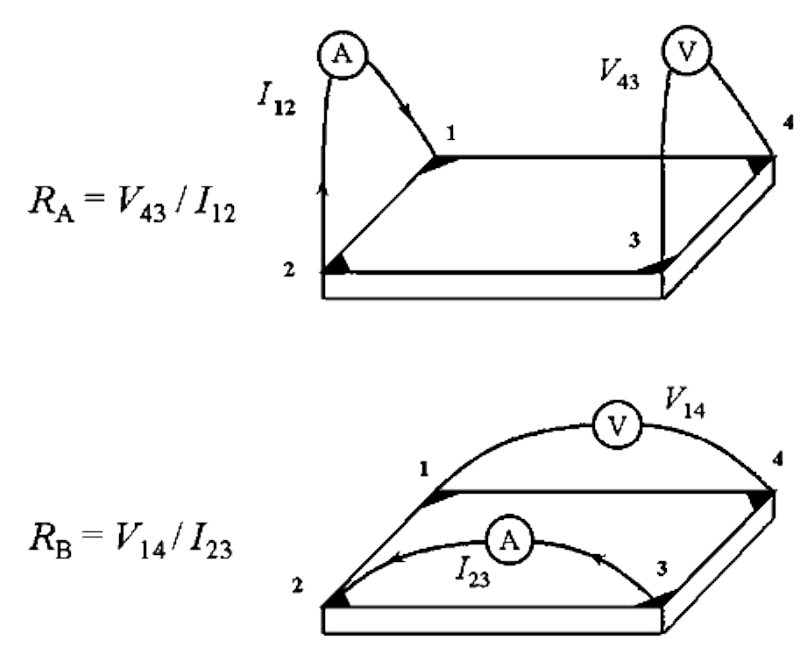

Figure 3.5: Schematic of a Van der Pauw configuration used in the determination of the two characteristic resistances $R_{A}$ and $R_{B}$

By considering these two resistance measurements $R_{A}$ and $R_{B}$, the actual sheet resistance is related to these resistances by the Van der Pauw formula by,

$$
e^{-\pi R_{A} / R_{S}}+e^{-\pi R_{B} / R_{S}}=1
$$

In general, it can be assumed that $R_{A}=R=R_{B}$. In this case, the sheet resistance is given by [136]

$$
R_{s}=\frac{\pi R}{\ln 2}
$$




\subsubsection{Hall mobility measurements}

Lorentz force is the underlying basic physical principle of the Hall Effect. When an electron moves along a direction perpendicular to an applied magnetic field, it experiences a force acting normal to both directions and moves in response to this force and the force affected by the internal electric field. The Lorentz force is given by

$$
\vec{F}_{\text {Lorentz }}=q[\vec{E}+(\vec{v} \times \vec{B})]
$$

The Hall effect is illustrated in figure 3.6 for a sample on a substrate. Here the charge is carried by electrons. A constant current $I_{13}$ flows through the sample and the entire sample is subject to a uniform magnetic field B perpendicular to the current flow. Electrons which are travelling through a magnetic field are subject to an upwards Lorentz force. This leads to a buildup of negative charge on one side of the sample and positive charge on the other due to the lack of electrons. This leads to a potential difference, which called the Hall voltage $V_{H}$ between the two sides of the sample. The objective of the Hall measurement in the Van der Pauw technique is to determine the sheet carrier density $n_{s}$ by measuring the Hall voltage $V_{H}$. The Hall voltage measurement consists of a series of voltage measurements with a constant current $I_{13}$ and a constant magnetic field B. 

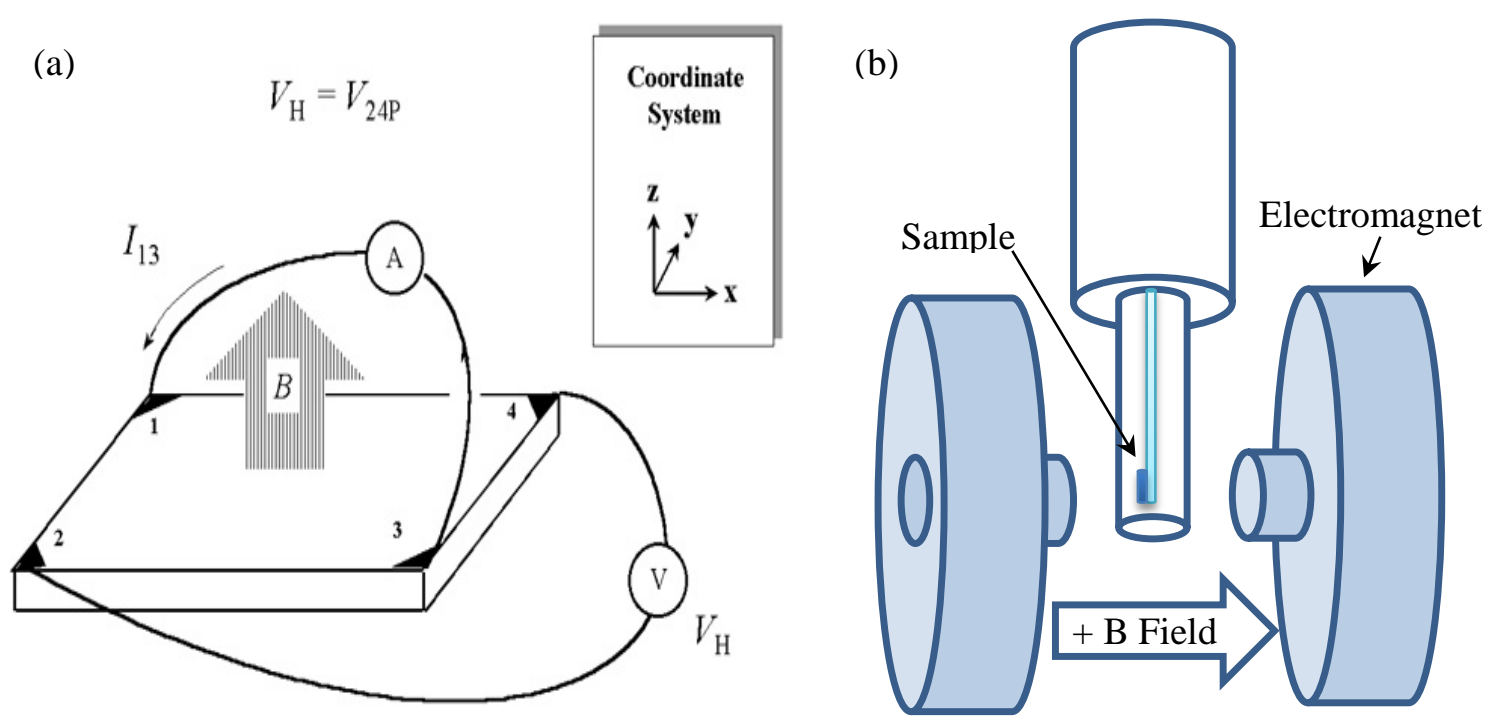

Figure 3.6: Schematic of (a) Van der Pauw configuration used in the determination of the Hall voltage $V_{H}$. (b) the sample placed in the magnetic field.

This transverse voltage is the Hall voltage $V_{H}$ and its magnitude is equal to

$$
\left|V_{H}\right|=\frac{I B}{q n d}
$$

Sheet density given by this equation,

$$
n_{s}=\frac{I B}{q\left|V_{H}\right|}
$$

Then Hall mobility of the sample can be calculated by,

$$
\mu=\left|V_{H}\right|{ }_{R_{S} I B}=1 /\left(q n_{s} R_{s}\right)
$$




\section{3 in-situ functionalization of graphene}

Availability of large-area CVD-grown Graphene facilitates straightforward S measurement on the Graphene without undergoing any micro-fabrication processes[137]. The Graphene specimens (monolayers) utilized in this work were synthesized by the chemical vapor deposition (CVD) method at low pressure $(\sim 100$ mT) on polycrystalline $\mathrm{Cu}$ foils and then transferred onto $\mathrm{SiO}_{2} / \mathrm{Si}$ substrates $[138$, 139], as described in chapter 02. A custom-designed split ring capacitively coupled RF plasma system (13.56 MHz, Max. power $600 \mathrm{~W}$ ) was used in room temperature. Transport measurements were done on the pristine graphene sample before functionalizing with hydrogen. The $S$ and $R$ of Graphene were measured over a temperature range of $300 \mathrm{~K}$ to $550 \mathrm{~K}$. For low temperature measurements, the chip carrier supporting the Graphene sample was transferred to a closed cycled refrigerator (Janis Research Co. CCS-350ST-H) which can be cooled down to a base temperature of $\sim 6 \mathrm{~K}$. Also the sample-containing refrigerator column is enclosed by an electromagnet (LakeShore Model EM4-CV 4-inch gap, Horizontal Field) capable of producing magnetic field which can be swept between -1 and +1 Tesla as in figure 3.7. $\mu$ was measured in Hall probe configuration by applying a perpendicular magnetic field. Hall voltage, $V_{H}$ was measured under $+1,0$, and -1 Tesla magnetic fields and corrections were made for parasitic voltages by averaging. 
(a)

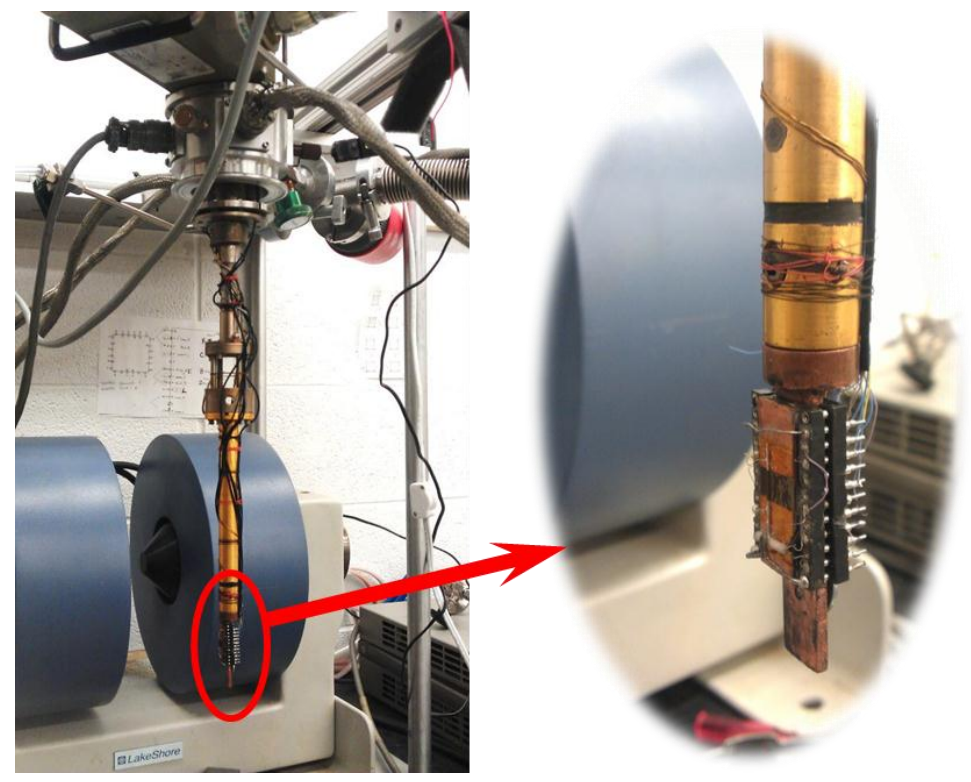

(b)

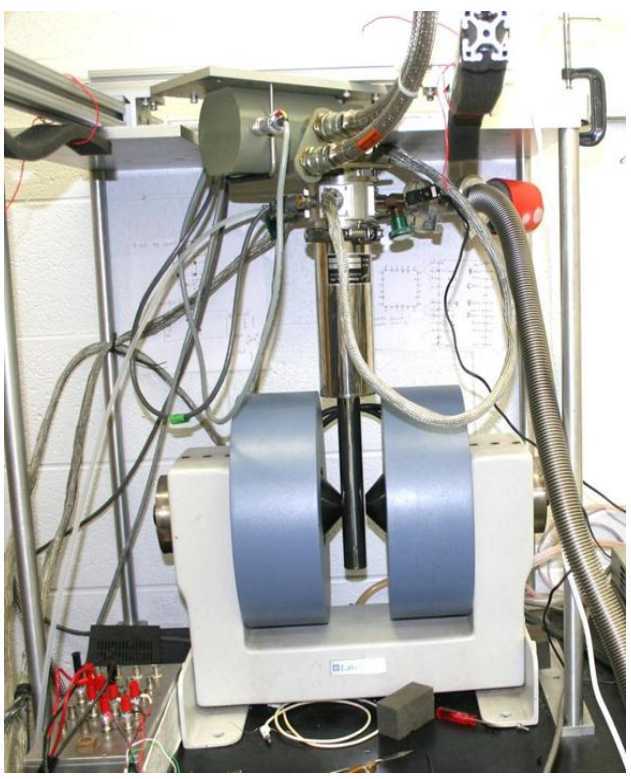

Figure 3.7: Low temperature transport measurement setup (a) Transferred sample to the cold head (b) Clod head and the sample covered with the vacuum shroud placed in between the electromagnet.

Once the transport measurements were done on the pristine graphene, sample will be trasferred to the functionalization chamber. In order to drive Graphene gradually through metal-insulator transition, it was sequentially hydrogenated in a highly controllable manner for different hydrogen concentrations. This functionalization process carried out while measuring transport properties in-situ. This enables to monitor the changes in sample properties while sample is still inside the chamber. Figure 3.8 shows plasma functionalization setup. This setup consists of controlled gas delivery system, reactor area, gas removal system etc. Hydrogen gas is supplied to the system at a $5 \mathrm{sccm}$ flow rate and chamber pressure is maintained at 10torr. 


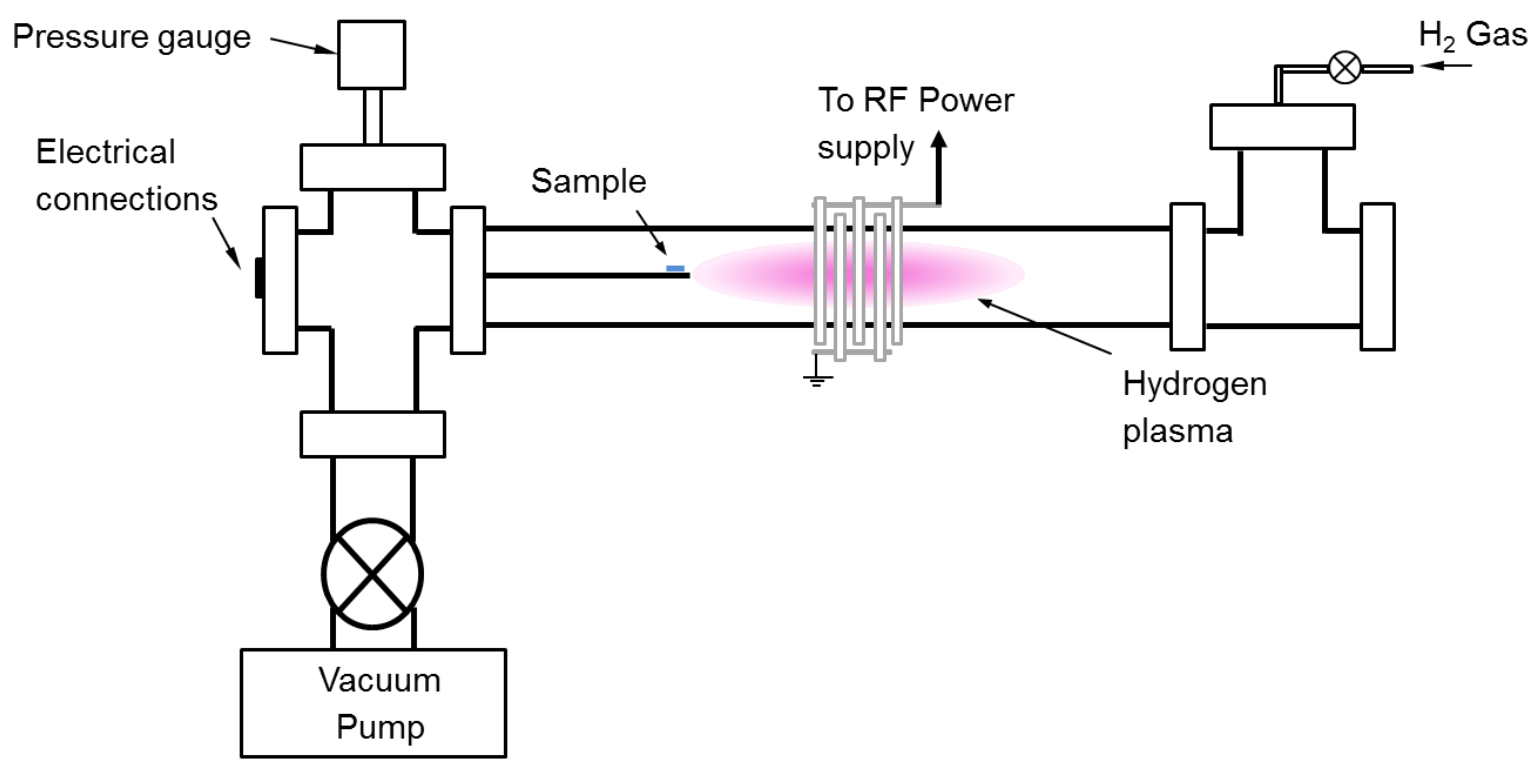

Figure 3.8: Schematic of the plasma functionalization setup

Plasma exposure time was established by in-situ monitoring of the change in resistance of the sample. Transport properties were measured by anchoring two miniature thermocouples $(\mathrm{Chromel}(\mathrm{KP}) / \mathrm{Au}-7$ at\%Fe $(\mathrm{Au}: \mathrm{Fe}))$ in $100 \mu \mathrm{m}$ diameter followed by two electrical concretions and a resistive heater on the sample as in Figure 3.2. Dc conductivity was measured with a standard four probes technique over a wide range of temperature. Graphene samples (typical size $\sim 4 \mathrm{~mm}$ x $4 \mathrm{~mm}$ ) were placed in a quartz reactor which has provisions for evacuation and exposure to hydrogen plasma at desired temperatures and pressures. 
(a)

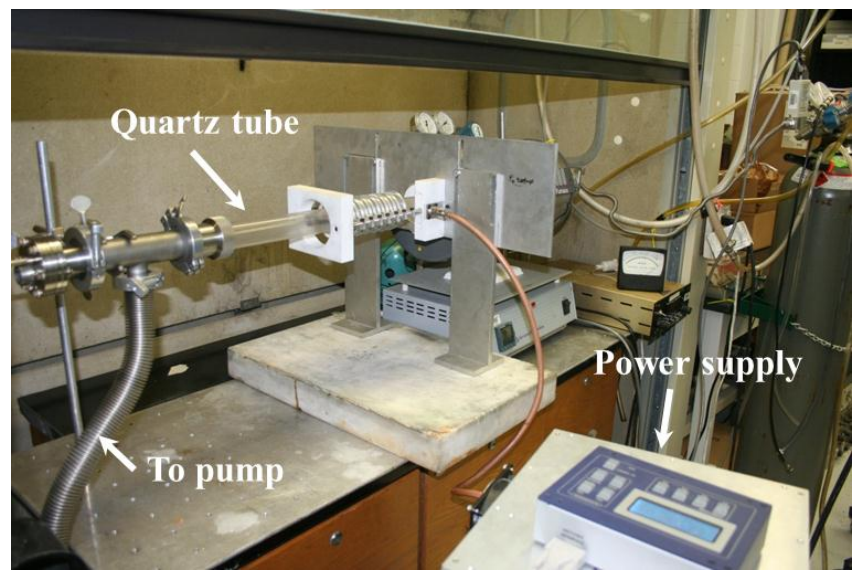

(c) (b)
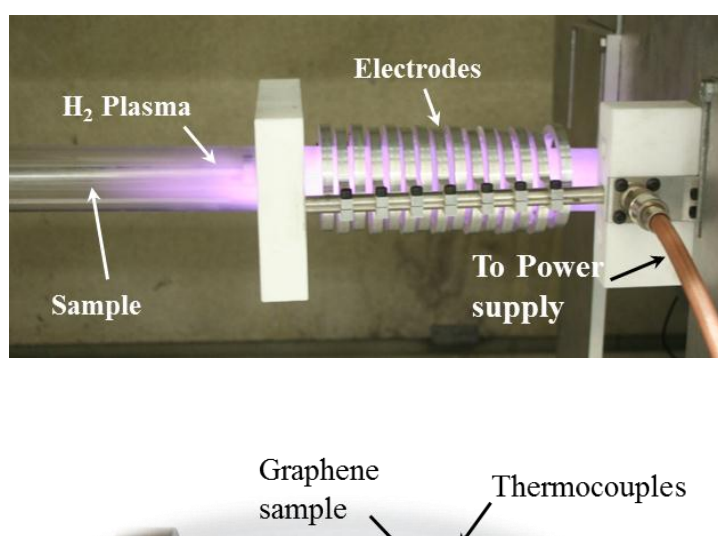

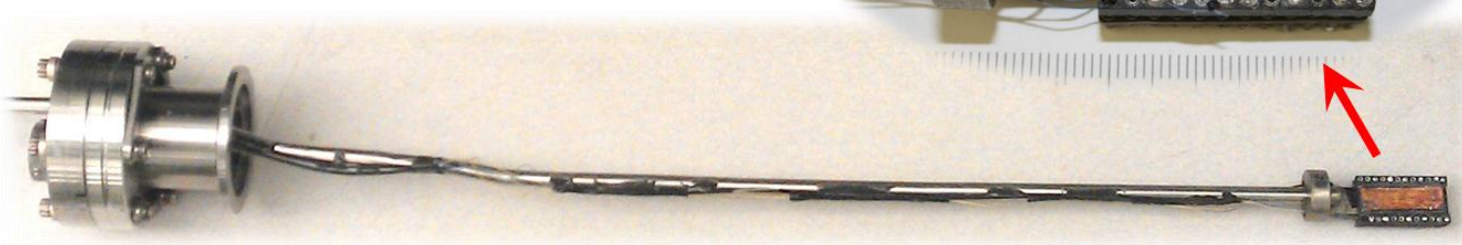

Figure 3.9: Plasma functionalization (a) setup (b) in-situ functionalization of graphene with $\mathrm{H}_{2}$ plasma (c) Graphene sample mounted on the probe.

The probe is loaded into a quartz reactor placed inside a tube furnace. The reactor is evacuated to a base pressure below $10^{-6}$ Torr using a turbo molecular pump and degassed at 500K, while the time evolution of the $S$ and the $R$ are recorded concomitantly. Sample was heated by wrapping heater around the quartz tube. Sample was continuously pumping throughout the process. Figure 3.9(a) shows a picture of the plasma functionalization setup. Once the sample is annealed, reactor is cooled down to room temperature under high vacuum. To measure the $S$, a temperature difference $(\Delta \mathrm{T}<1 \mathrm{~K})$ is generated across the sample by applying a voltage pulse to the heater on the sample. The typical heating power is $10 \mathrm{~mW}$, and the pulse duration is $3-5$ seconds. The slope of the thermo-emf $(\Delta V)$ versus temperature difference $(\Delta T)$ 
due to the heat pulse is used to obtain $\mathrm{S}$ at a given temperature. In order to measure the $\mathrm{R}$, an excitation current was applied through the two current leads and the voltage across the two thermocouple wires was measured in van der Pauw configuration. The excitation current level was kept extremely low to avoid joule-heating especially at low temperatures. During the annealing $S$ changed sign (indicating that the sample becomes n-type) to finally reach a saturated value of $S \sim-40 \mu \mathrm{V} / \mathrm{K}$. Concomitantly $\mathrm{R}$ increased and reached its maximum value when $S$ changed its sign[140] and then gradually decreased to a saturated value $\sim 1 \mathrm{k} \Omega$. The ambient p-type behavior has been identified as due to electrochemically mediated charge transfer mechanism between a redox couple in humid air and the Fermi-energy of Graphene[141].

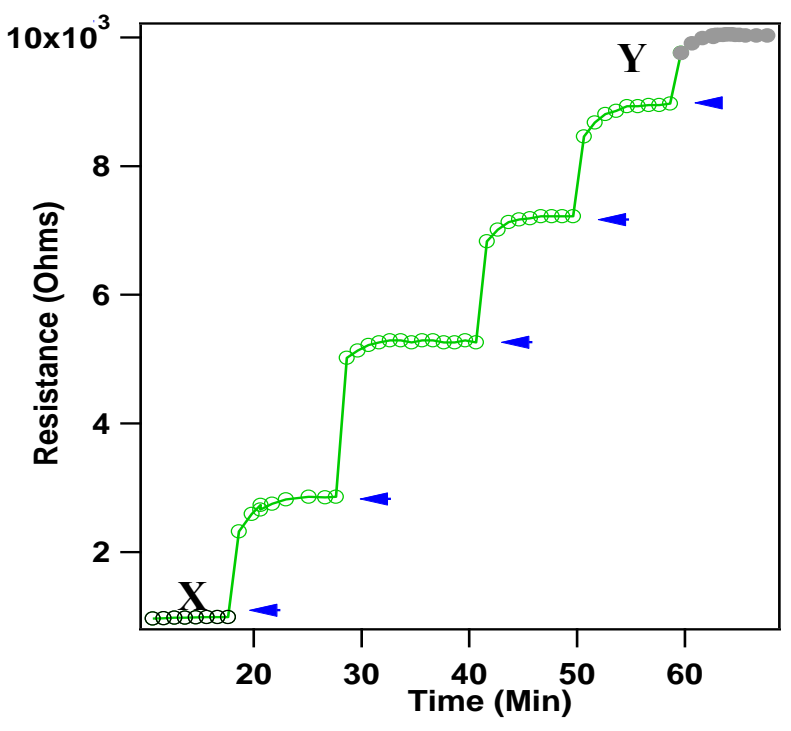

Figure 3.10: In-situ time evolution of the Resistance, $R(t)$ during hydrogenation. The arrow heads represent the initiation of intermittent plasma

Figure 3.10 shows the in-situ time evolution of resistance of the degassed Graphene during first hydrogenation process (starting at "X"). The Graphene sample was carefully exposed to the hydrogen-plasma for a short period of time ( $\sim 5$ seconds) and the plasma was then turned off. The monitoring of the change in resistance and 
thermoelectric power was continued. thermoelectric power was found to change to a positive value from the negative degassed state. The formation of $\mathrm{C}-\mathrm{H}$ bonding due to the presence of atomic hydrogen in the plasma reduces the delocalized electrons (depletion of electrons) in the Graphene network thereby inducing p-type doping. Extreme care was taken in order not to inflict any damages to the sample during the process. . As shown in Figure 3.10, $R$ increased gradually and saturated at a higher $R$ value. Then the plasma was ignited again for $\sim 5$ seconds, turned off, and monitored the time evolution of $R$. The intermittent plasma treatments are indicated by the arrows in Figure 3.10. This process was continued until the $R$ approached the desired value at "Y". once the functionalization step is completed, temperature dependence of the transport properties $(R, S, \mu)$ were measured for that particular degree of hydrogenation. 


\section{4 ex-situ characterization of functionalized graphene}

\subsubsection{Low temperature transport measurements}

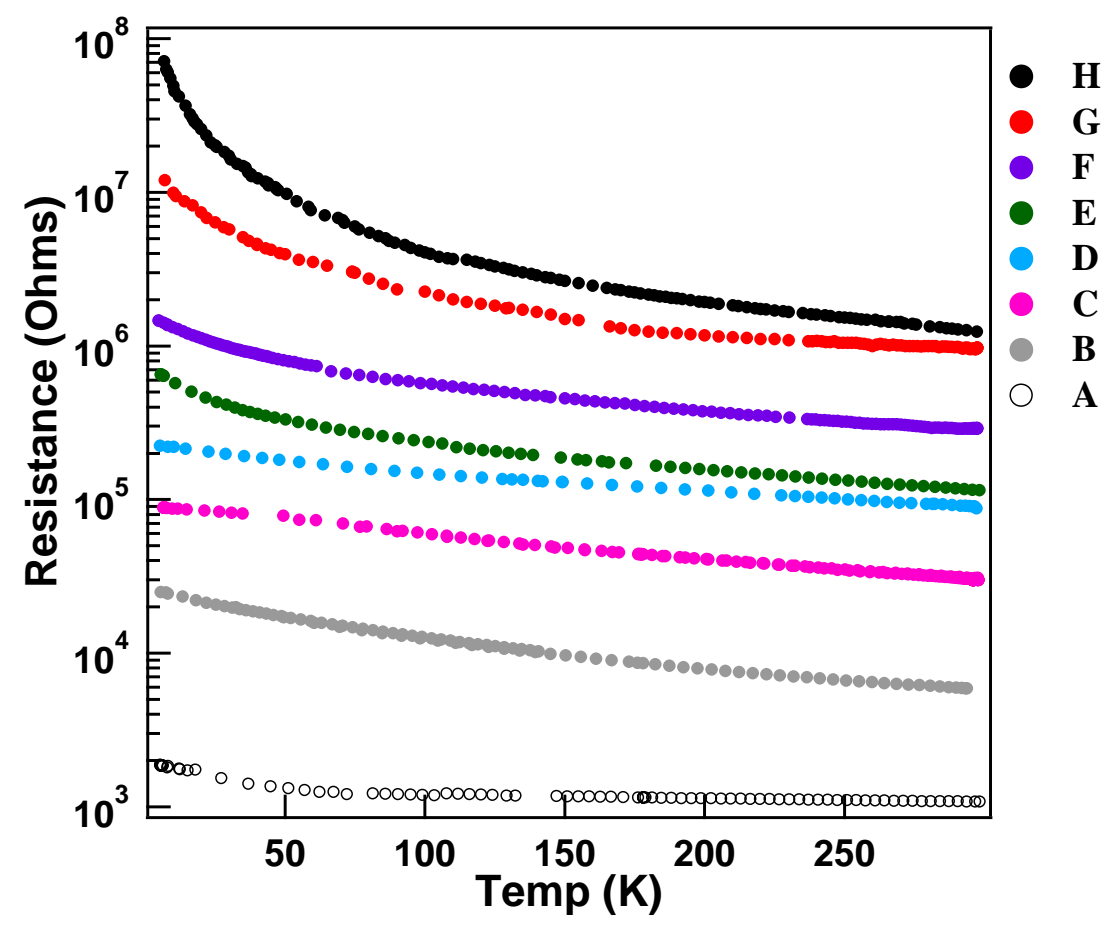

Figure 3.11: Temperature dependence of resistance, $R(T)$ of progressively hydrogenated Graphene samples.

Temperature dependence of the resistance $(R)$ was measured for that particular degree of hydrogenation as shown in figure 3.11 (transition of low-disordered graphene to high-disordered graphene). The temperature dependence of the resistance after first hydrogenation is represented by the curve " $\mathrm{B}$ " in Figure 3.11. The process was repeated until resistance was no longer measurable. Resistance starts growing monotonically as the temperature decreases in upper curves. This is the characteristic behavior of an insulator. The $R(T)$ curves after each hydrogenation process are shown in Figure 3.11 labeled from " $\mathrm{B}$ " to " $\mathrm{H}$ ". This behavior strongly suggests the existence 
a metal-insulator transition in 2D system (graphene) at zero magnetic field, contradicting the theoretical predictions by Abrahams[142].

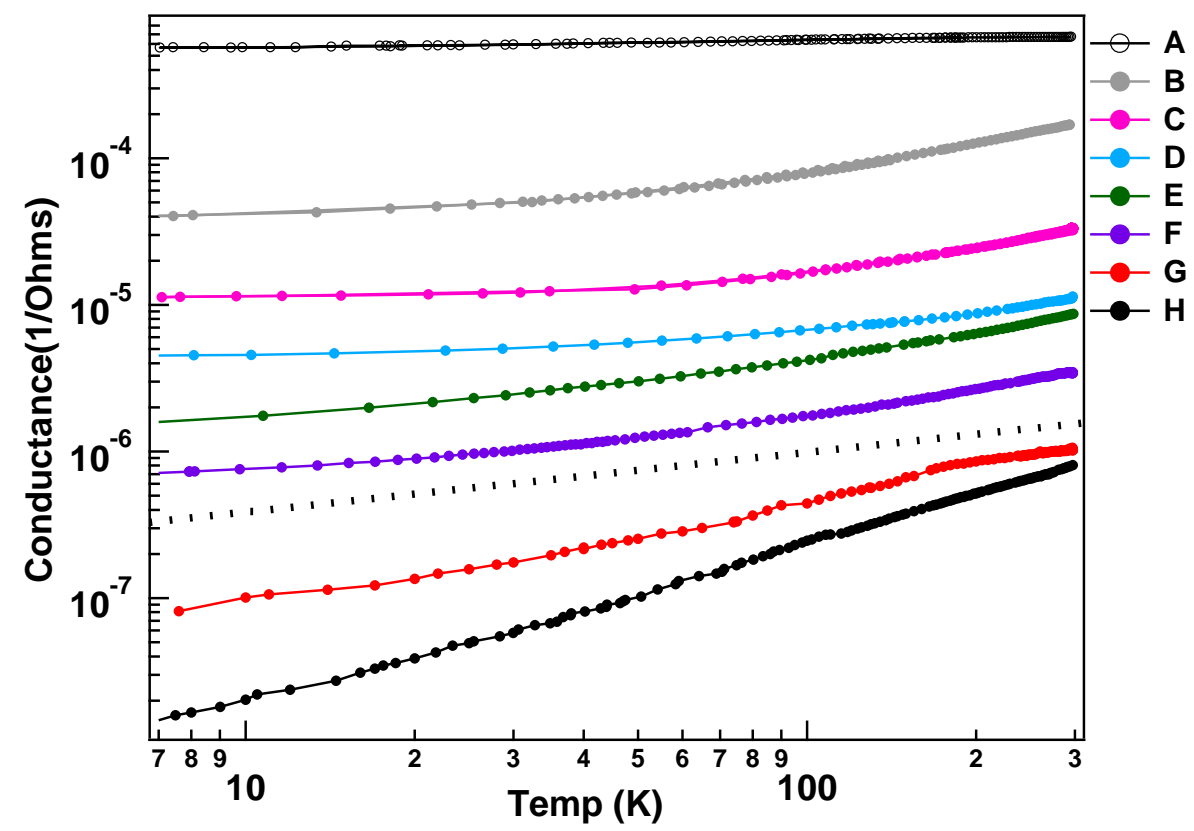

Figure 3.12: Temperature dependence of conductance, $G(T)$ in logarithmic scale for progressively hydrogenated Graphene samples. The dotted line separates metallic samples from insulating.

Figure 3.12 shows the logarithmic dependence of the conductance, $G(\equiv 1 / R)$, vs. the logarithmic temperature. This plot helps to separate metallic samples, for which $G$ approaches a constant value at low temperatures, from insulating samples, for which $G$ falls rapidly at low temperatures. Samples with conductivity curves above the dashed line ("A" to " $\mathrm{F}$ ") remain metallic because the conductance remains nonzero for $T \rightarrow 0$, whereas samples with conductance curves below it ("G" and "H") seem to exhibit insulating behavior for $T \rightarrow 0$. The sample represented by "G" appears to be close to the transition. 


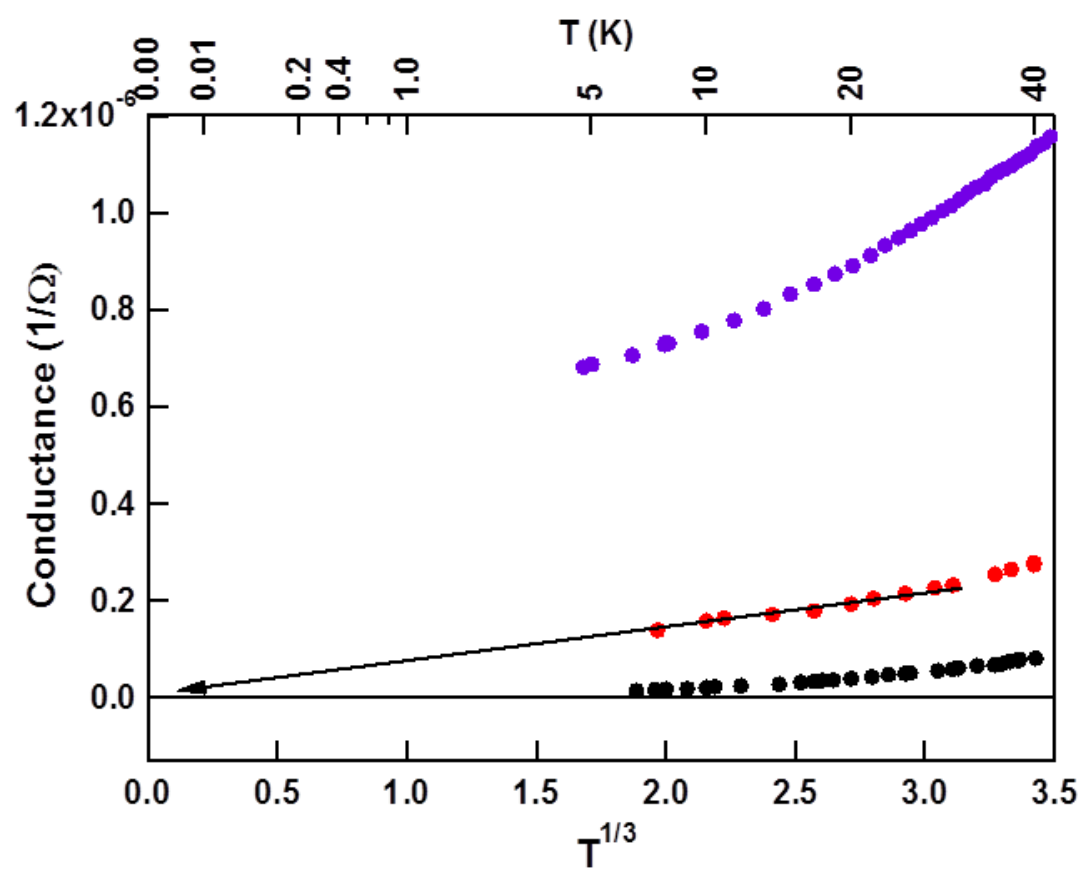

Figure 3.13: Low temperature behavior of the conductivity, $G$ vs $T^{1 / 3}$ plot for the three curves F, G, and $\mathrm{H}$ representing metallic, critical, and insulating samples respectively. Notice that the curve $\mathrm{G}$ clearly approaches zero linearly as a function of $\mathrm{T}^{1 / 3}$

To elaborate the critical behavior we plot conductance, $G$ vs $T^{1 / 3}$ in an expanded scale in the vicinity of $G=0$ in Figure 3.13. Closer inspection shows that the data near the MI transition are actually better described by a $T^{1 / 3}$ dependence for low $T$. As seen in Figure 3.13, the conductance for the middle curve ("G") varies almost linearly as a function of $T^{1 / 3}$ for temperature below $30 \mathrm{~K}$. this can me confirms by extrapolating the curve $\mathrm{G}$ to $\mathrm{T}=0$. Samples with conductance curves above it ("F") remain metallic because the conductance remains nonzero for $T \rightarrow 0$ whereas samples with conductivity curve below it ("H") will not conduct for $T=0$ as conductivity begins to vanish at $T \sim 10 \mathrm{~K}$ [143-145]. 


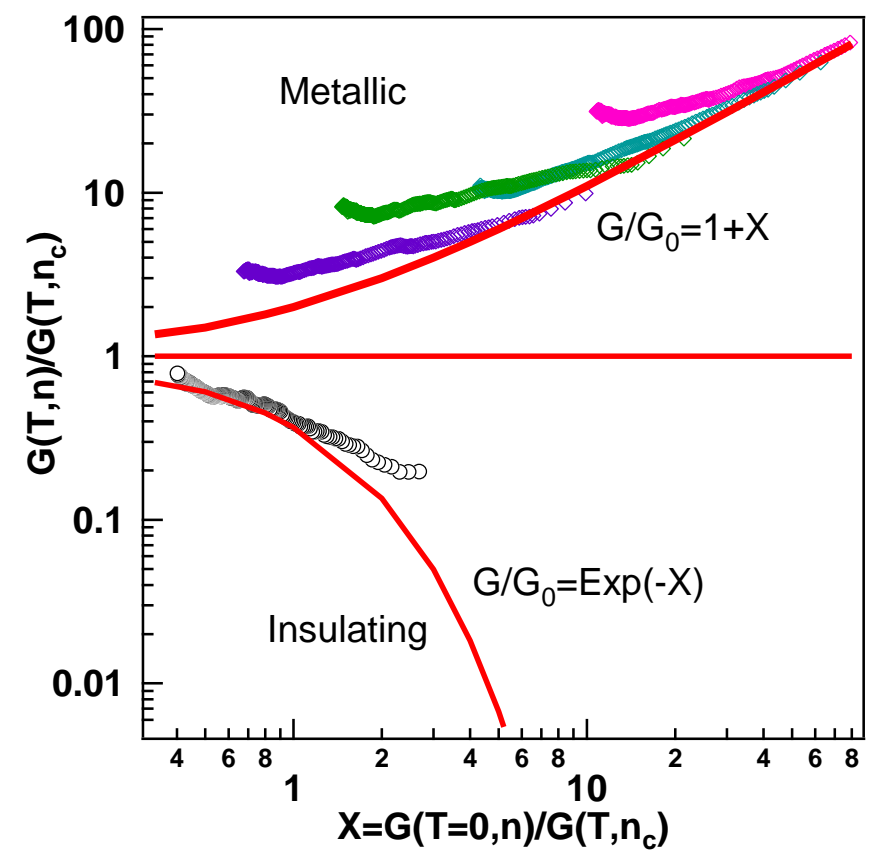

Figure 3.14: Scaling Plot of the conductivity data. Data on the metallic side collapse onto one curve and can be fitted with $G / G_{0}=1+X$, where $X=G(T=0$, $n) / G\left(T, n_{c}\right)$.

The temperature dependence of conductivity for each hydrogenation can be used to test the existence of a scaling function provided that we have the data for the critical concentration of hydrogen, $n_{c}$. The hydrogenation process represented by " $\mathrm{G}$ " with conductance approaching zero almost linearly with $T^{1 / 3}$ (Figure 3.13) is considered to correspond to $n_{c}$. In Figure 3.14, we plotted the temperature dependence of conductance, $G(T, n)$ divided by the data for critical concentration, $G\left(T, n_{c}\right)$ as a function of $X=G(T=0, n) / G\left(T, n_{c}\right)$, where $G(T=0, n)$ is the saturated conductivity value at lowest attainable temperature for the metallic samples. In fact $X$ can be interpreted as the ratio between the localization length, $\xi$ and the temperature-determined characteristic length, $l_{T}$ as $\mathrm{X} \equiv l_{T} / \xi$ [143]. It can be seen that all the data on the metallic side collapse into one curve and can be fitted with the simple scaling function $G / G_{0}=1+X$, where $G_{0}$ is a constant. On the insulating side, the data is expected to 
collapse to a single curve again by dividing by the critical data set. The one data set obtained for the insulating side can be approximated with the scaling function, $G / G_{0}=\operatorname{Exp}(-X)$

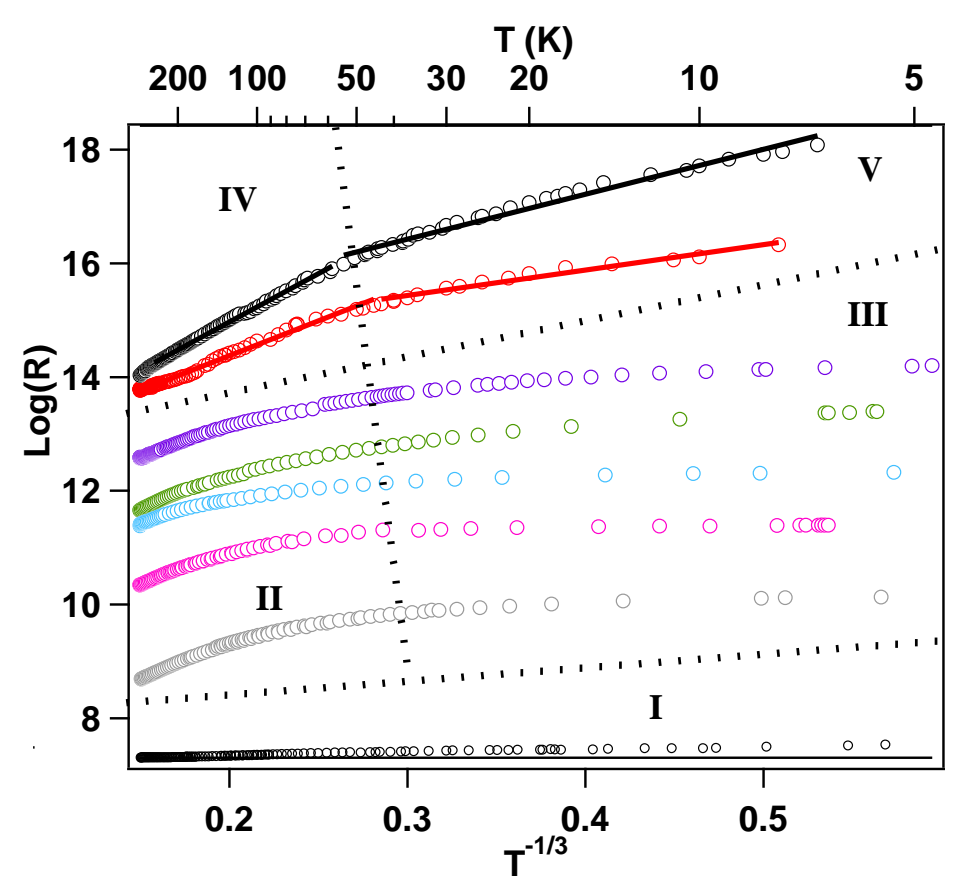

Figure3.15: Variable range hopping analysis for $R(T)$

The temperature dependence of the $R$ of the hydrogenated Graphene shows the negative temperature coefficient of the $R(d R / d T<0)$ in the whole investigated temperature range $(8-300 \mathrm{~K})$ as seen in Figure 3.15. It is known that the temperature dependence of the conductance, $G(T)$ follows the $G(T) \sim \ln (T)$ dependence in the 2D weak localization (WL) which could be applicable for pristine Graphene. But for hydrogenated Graphene, electrical transport is expected to be governed by hopping conduction. Assuming possibility of the 2D-VRH conduction in our system due to hydrogenation of carbon atoms, we fitted $R(T)$ dependence by classical law for variable range hopping $(\mathrm{VRH})$ 


$$
R=R_{0} \operatorname{Exp}\left(\frac{T_{0}}{T}\right)^{1 / 3}, \text { where } T_{0}=\frac{13.8}{k_{B} N\left(E_{F}\right) \xi^{2}}
$$

$k_{B}$ is the Boltzmann constant, $N\left(E_{F}\right)$ is the density of states at the Fermi energy, $\xi$ is the localization length[146].

In this context we plot Logarithmic $R, \log (R)$ vs $T^{-1 / 3}$ in Figure 3.15. It is evident from the plot that the data for highly hydrogenated Graphene samples fit very well for the 2-D VRH theory for low temperature regime (below $\sim 40 \mathrm{~K}$ ). For higher temperatures, another clear linear range for $T^{1 / 3}$ dependence is evident. But for dilute hydrogenation, VRH theory seems to deviate from the experimental data especially for higher temperature.

In Figure 3.15, we identify several regions of interest with unique characteristic signatures. Region I: transport properties of degassed Graphene are governed by electron diffusion with metallic behavior; region II: metallic behavior with electron diffusion leading to WL phase 1 for dilute hydrogenated Graphene at higher temperatures; region III: metallic behavior with electron diffusion leading to WL phase 2 for dilute hydrogenated Graphene at lower temperature; region IV: insulating behavior with electron transport governed by VRH leading to strong localization (SL) Phase 1 for heavily hydrogenated Graphene at higher temperatures ; region $\mathrm{V}$ : insulating behavior with VRH governed transport leading to SL phase 2 for heavily hydrogenated Graphene at lower temperatures. 


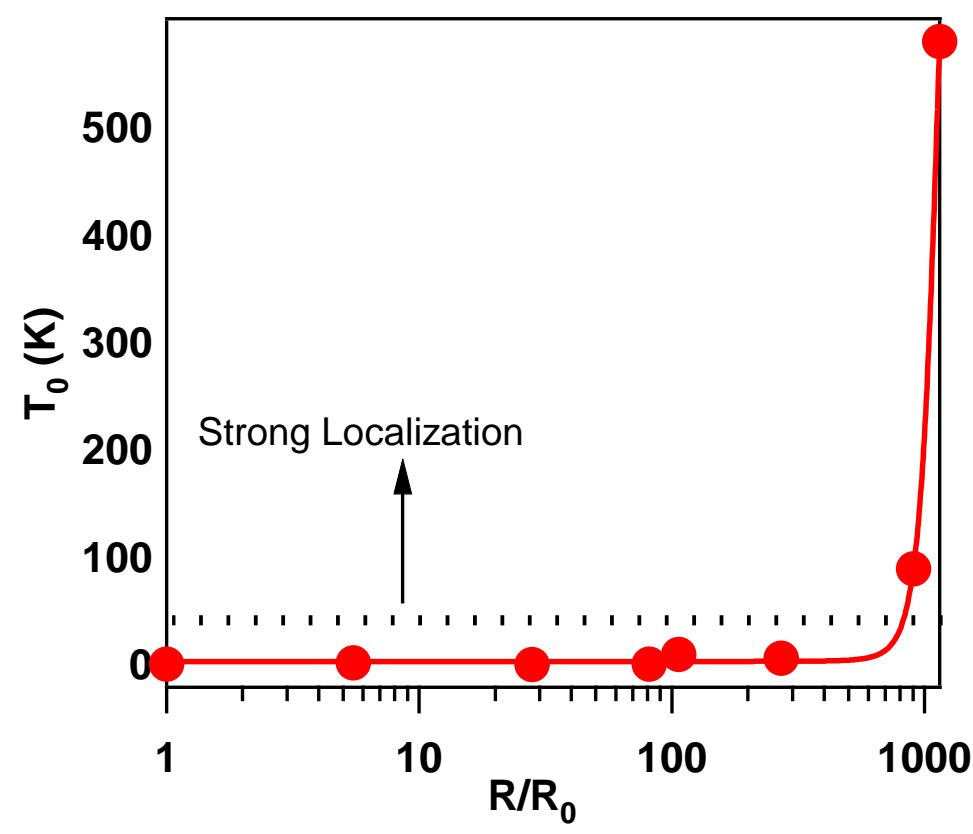

Figure 3.16: $T_{0}$ values (extracted from VRH fitting) for each hydrogenation process characterized by change in room temperature resistance

Figure 3.16 shows the $T_{0}$ value extracted from VRH analysis at low temperatures as a function of hydrogenation represented by the change in the room temperature resistance, $R / R_{0}$, where $R$ and $R_{0}$ are the room temperature resistances of Graphene after each hydrogenation process and prior to hydrogenation (vacuum annealed) respectively. For the first few hydrogenation processes, $T_{0}$ remains small $(<10 \mathrm{~K})$ implying VRH is not the dominant mechanism of electron transport. However, for the last 2 hydrogenation processes, $T_{0}$ increases above $90 \mathrm{~K}$ for a value as high as $575 \mathrm{~K}$. Sudden increase of $T_{0}$ marks the transition from $W L$ to $S L$. 


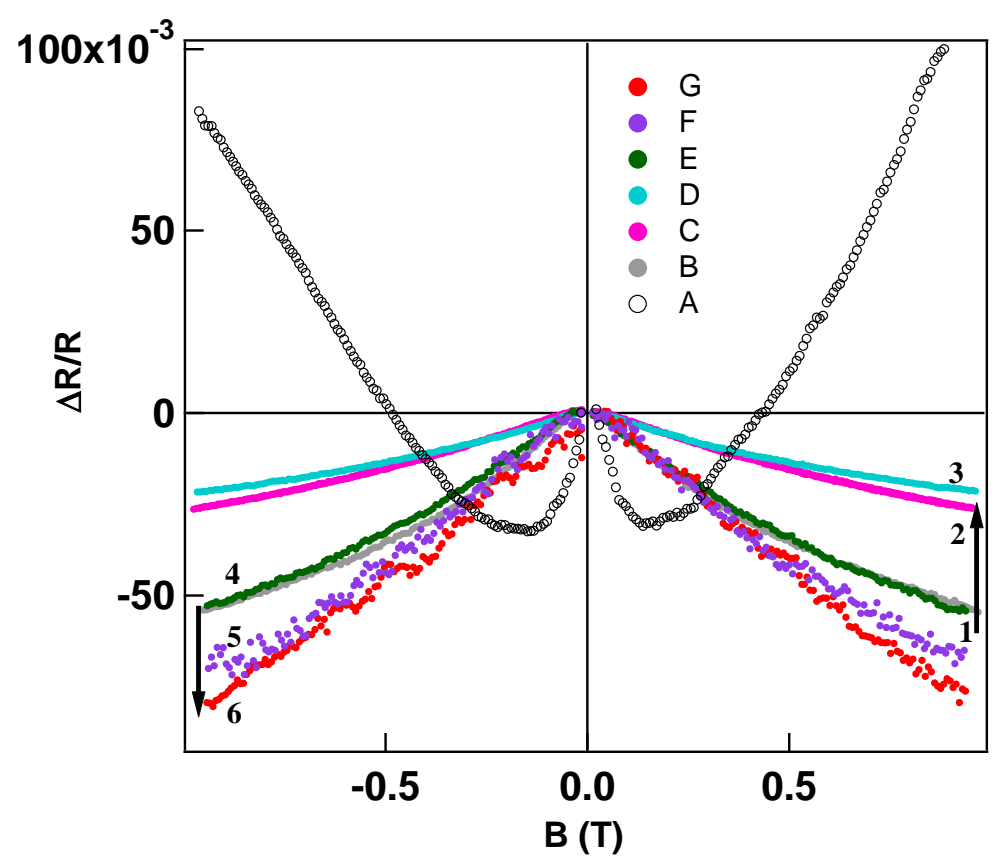

Figure 3.17: Magneto resistance data for progressively hydrogenated Graphene. Data for the untreated Graphene is also shown.

Figure 3.17 shows the $M R$ data for hydrogenated Graphene at $8 \mathrm{~K}$. The negative $M R$ in the low magnetic fields range was observed. The degassed Graphene sample shows low field negative $M R$, and governed by classical $B^{2}$ dependence for high magnetic field. There is an asymmetry in the data presumably due to the contact misalignments. After first hydrogenation, $M R$ is entirely negative and remarkably symmetric. In the subsequent 2 hydrogenation processes the $M R$ is seen to suppress, but start to enhance for further hydrogenation. Results from first 3 hydrogenation processes are labeled 1-3 with an upward arrow to indicate the decrease of MR while the next 3 hydrogenation processes are identified as 4-6 with a downward arrow to indicate the increase of $M R$. The negative $M R$ is inherent for the systems where conductivity can be described in the framework of $W L$ theory [131, 147-149]. The low temperature data are analyzed according to $W L$ theory developed for Graphene. The quantum mechanical correction, $\delta \sigma$ to the classical Drude conductivity is given by, 


$$
\delta \sigma=\frac{e^{2}}{\pi h}\left[F\left(\frac{\tau_{B}^{-1}}{\tau_{\phi}^{-1}}\right)-F\left(\frac{\tau_{B}^{-1}}{\tau_{\phi}^{-1}+2 \tau_{\text {inter }}^{-1}}\right)-2 F\left(\frac{\tau_{B}^{-1}}{\tau_{\phi}^{-1}+\tau_{\text {inter }}^{-1}+\tau_{\text {int } r a}^{-1}}\right)\right]
$$

Here, $F(x)=\ln (x)+\psi\left(\frac{1}{2}+\frac{1}{x}\right)$, where $\psi$ is the digamma function, $B_{x}=\frac{\hbar}{4 e D \tau_{x}}$ with $x=\phi$, inter, and intra; $\tau_{\phi}{ }^{-1}=L_{\phi}{ }^{2} / D$ is the phase coherence time,

$L_{\phi}$ is the phase coherence length. The diffusion constant $D$ is expressed as,

$$
D=\frac{1}{2} v_{F}^{2}\left(\frac{1}{\tau_{\text {inter }}}+\frac{1}{\tau_{\text {intra }}}\right)
$$

Where $v_{F} \sim 10^{6} \mathrm{~m} / \mathrm{s}$ is the Fermi velocity

Then the magnetoresistance [150],

$$
\begin{gathered}
\frac{\Delta R}{R}=\frac{R(B)-R(B=0)}{R(B=0)}=-R_{s} \delta \sigma(B) \text { is given by, } \\
\frac{\Delta R}{R}=\frac{-e^{2} R_{s}}{\pi h}\left[F\left(\frac{B}{B_{\phi}}\right)-F\left(\frac{B}{B_{\phi}+2 B_{i_{\text {nter }}}}\right)-2 F\left(\frac{B}{B_{\phi}+B_{\text {inter }}+B_{i_{\text {ntra }}}}\right)\right]
\end{gathered}
$$

Where, $R_{s}$ is the sheet resistance of the Graphene film. 
(a)

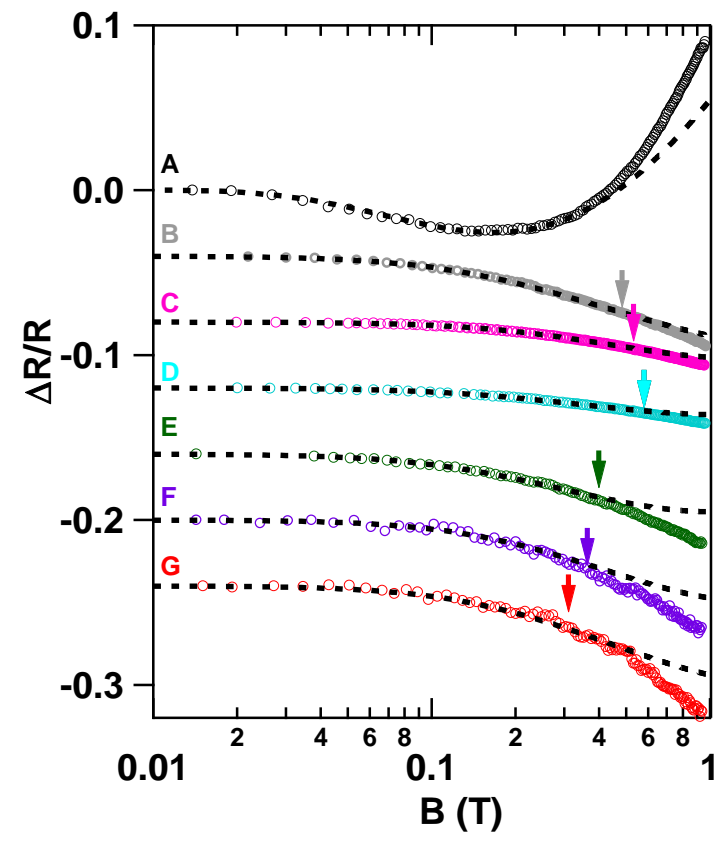

(b)

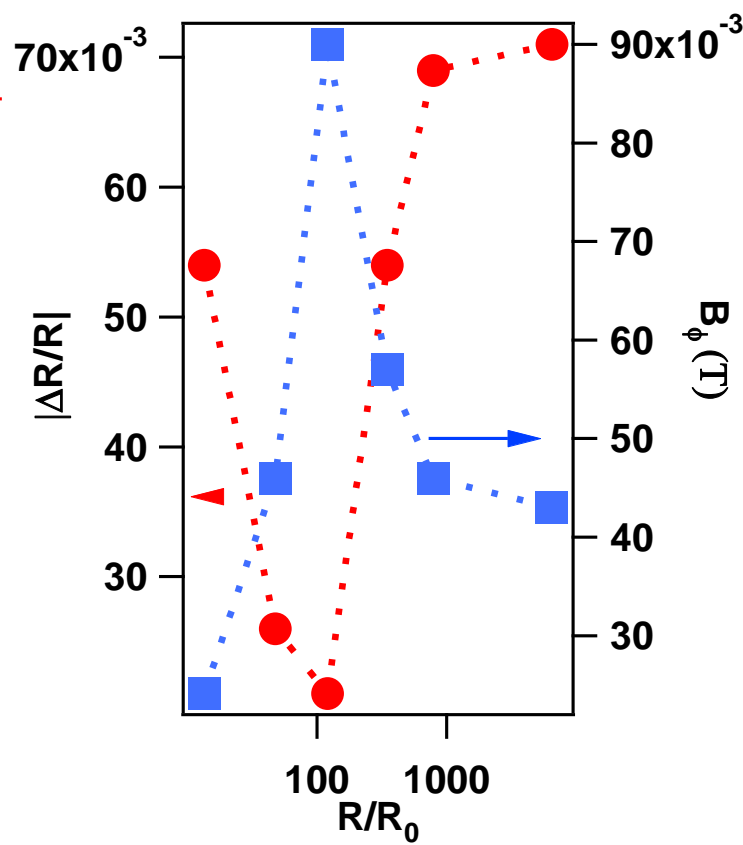

Figure 3.18: (a) Magneto resistance $(\Delta R / R)$ data for progressively hydrogenated Graphene with the best fit for $W L$ theory at low magnetic field values. Each data set is offset for clarity. (b) Normalized Magnetoresistance (MR) (left axis) and dephasing magnetic field, $B_{\phi}$ (right axis) for progressive hydrogenation characterized by change in the room temperature resistance

Figure 3.18(a) shows the $M R$ data with the best fit for $W L$ theory describes by eq.(2) at low magnetic fields. Each data set is offset for clarity. The $M R$ for the degassed pristine Graphene is also shown for comparison. $M R$ is seen to increase as the hydrogenation progresses for the first 3 hydrogenation processes. The $W L$ theory fits very well for magnetic fields up to $\sim 0.8 \mathrm{~T}$. The extracted dephasing length decreases from $\sim 60 \mathrm{~nm}$ to $\sim 30 \mathrm{~nm}$. During the next hydrogenation processes $M R$ starts to increase and the theory of $W L$ fails to account for the data above $0.2 \mathrm{~T}$. The trend of $M R$ is shown in Figure 3.18(b). This behavior is a signature for the gradual transition from $W L$ to $S L$. WL is traditionally associated with the interference of electron wavefunctions in disordered electronic systems traversing self-crossing paths in which an electron can propagate in the clock-wise and counter-clockwise direction 
around a loop. Low-field negative $M R$ due to changing of phase between alternate hopping paths enclosing a magnetic flux has been predicted for systems with hopping conductivity mechanism as well[151].

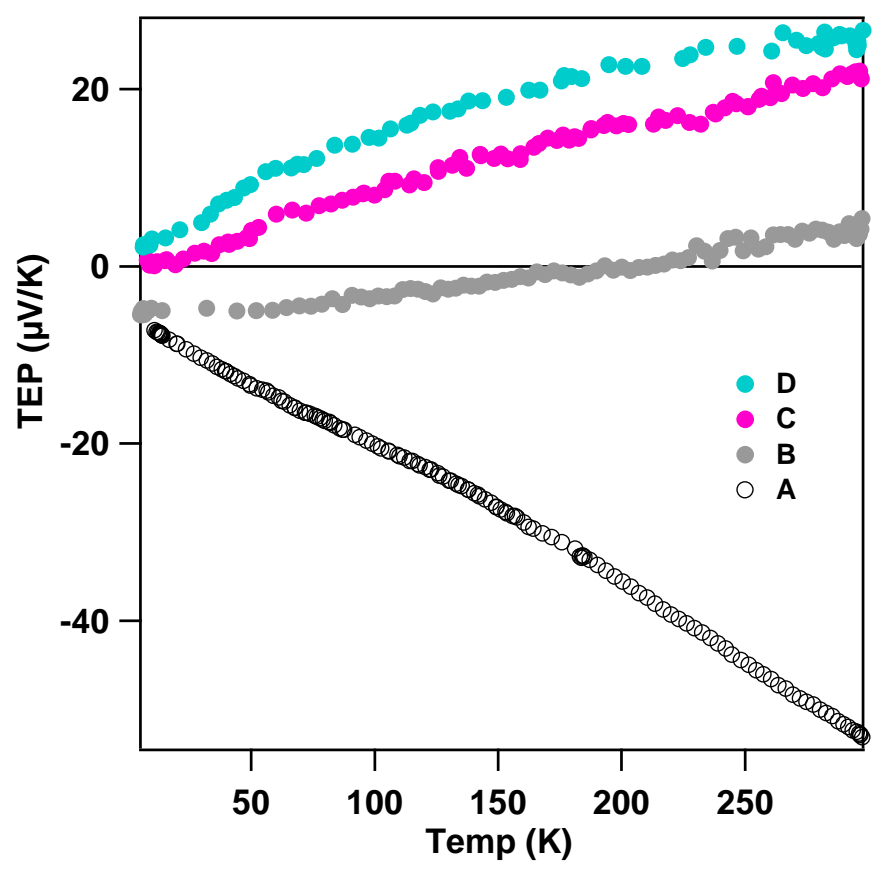

Figure 3.19: Temperature dependence of Thermoelectric power, $S(T)$

Figure 3.19 shows the temperature dependence of $S$ of pristine Graphene after degassing and after being subjected to series of controlled hydrogenation processes. The air exposed Graphene (CVD grown) is known to be p-type, but becomes n-type after degassing under vacuum at temperatures $\sim 200{ }^{0} \mathrm{C}$. The degassed Graphene sample shows a nearly linear temperature dependence of $S$ over the entire temperature range and remains n-type. Even after a short hydrogenation process with a nearly twofold increase in its initial $R$ value, the $S$ turns slightly positive to $\sim 5 \mu \mathrm{V} / \mathrm{K}$. Even after exposure to air at this point, the $S$ remains unchanged. As seen in Figure 3.19, the temperature dependence of $S$ of this dilute hydrogenated Graphene changes from positive to negative below $\sim 200 \mathrm{~K}$ and it remains negative in the entire temperature range below $\sim 200 \mathrm{~K}$. This behavior is not well understood at present and requires a 
detailed study. However, such temperature dependence of $S$ has been observed for polyaniline derivatives and attributed to the existence of a half-filled polaronic band situated deep in the energy gap of polyaniline[152]. In the next hydrogenation, $S$ increases to $\sim+15 \mu \mathrm{V} / \mathrm{K}$ in correspondence with an order of magnitude increase of $R$. The temperature dependence of $S$ for this sample remains p-type almost throughout the entire temperature range with metallic characteristics. The subsequent hydrogenation caused further increase of the $S$ and the temperature dependence showed similar metallic behavior, consistent with $R(T)$ data. Unfortunately the next hydrogenation caused resistance value high enough to make the $\mathrm{S}$ measurements impossible especially at low temperatures.

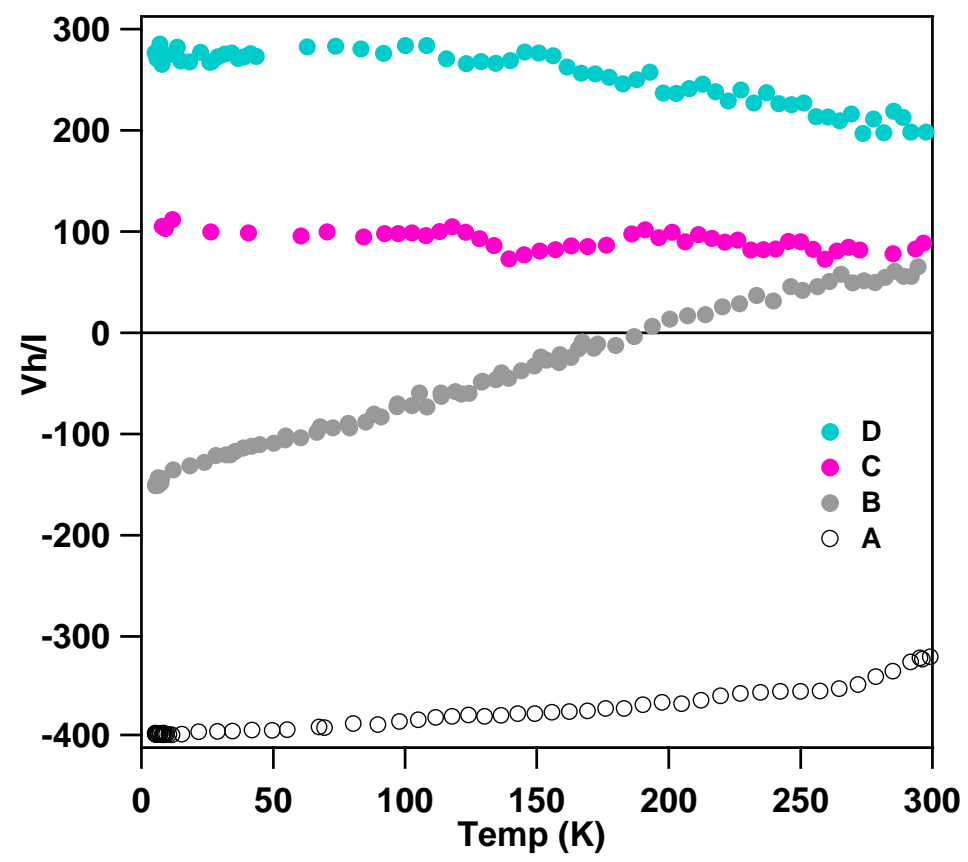

Figure 3.20: Temperature dependence of Hall voltage over excitation current, $V_{H} / I$ for progressively hydrogenated Graphene.

For direct comparison with $S(T)$ data, we plot in Figure 3.20 the temperature dependence of Hall voltage divided by the excitation current, $I,\left(V_{H} / I\right)$, for pristine 
Graphene and after being subjected to the first 3 hydrogenation processes. For the degassed Graphene sample, $V_{H} / I \sim-400 \Omega$ and remains negative in the entire temperature range between $8 \mathrm{~K}$ and $300 \mathrm{~K}$ with only very slight change. After first hydrogenation $V_{H} / I$ becomes slightly positive $\sim+80 \Omega$ for temperatures above $200 \mathrm{~K}$ and the temperature dependence shows nearly linear behavior with $V_{H} / I$ becoming negative below $\sim 200 \mathrm{~K}$. This unusual behavior is in accordance with the temperature dependence of $S$ for this sample requires further investigation. In the next hydrogenation, $V_{H} / I$ increases further to $\sim 100 \Omega$ and the temperature dependence of $V_{H} / I$ for this sample remains p-type throughout the entire temperature range with almost no change. The subsequent hydrogenation caused further increase of $V_{H} / I$ and the temperature dependence showed similar metallic behavior. Unfortunately the next hydrogenation caused $\mathrm{R}$ value high enough to make the $V_{H}$ measurements impossible.

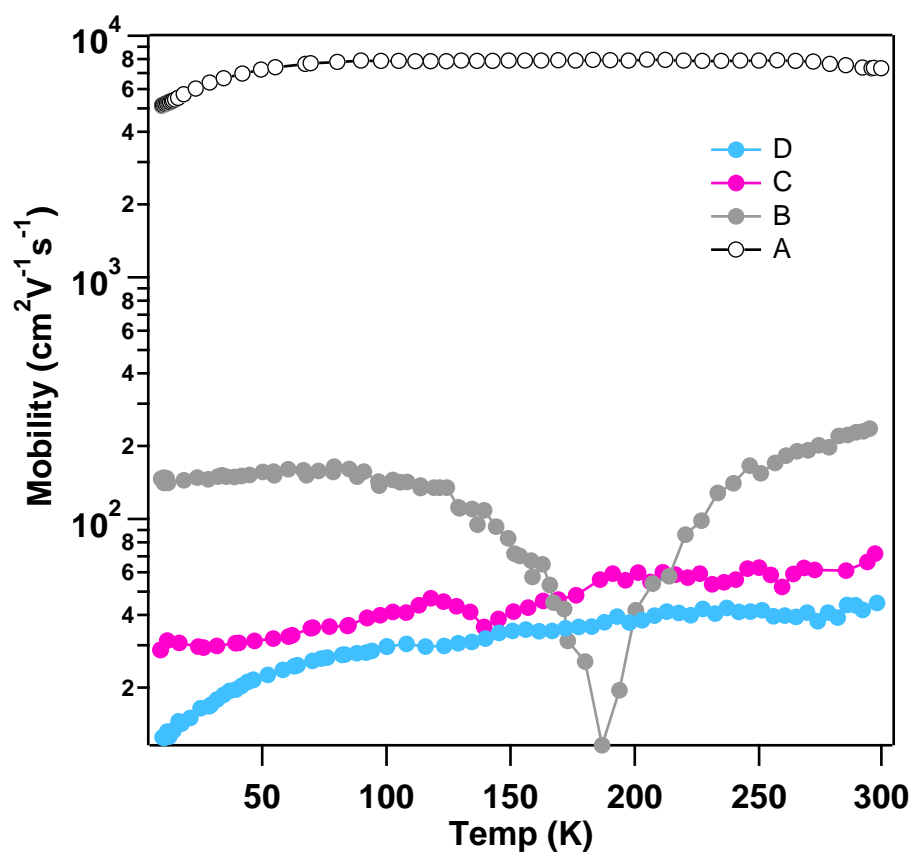

Figure 3.21: Temperature dependence of Hall Mobility $(|\mu|(T))$ for progressively hydrogenated Graphene. 
In order to compare the change of mobility as a consequence of hydrogenation, Figure 3.21 depicts the temperature dependence of $\mu,|\mu|(T)$ for the samples represented by A-D. It can be seen that $\mu \sim 7300 \mathrm{~cm}^{2} \mathrm{~V}^{-1} \mathrm{~s}^{-1}$ for degassed Graphene on glass substrate reduces after each hydrogenation, finally attaining a value as low a $\sim 40 \mathrm{~cm}^{2} \mathrm{~V}^{-1} \mathrm{~s}^{-1}$ after three sequential hydrogenation processes making it immeasurable after further treatment.

\subsubsection{Raman and XPS characterization of graphene}

(a)

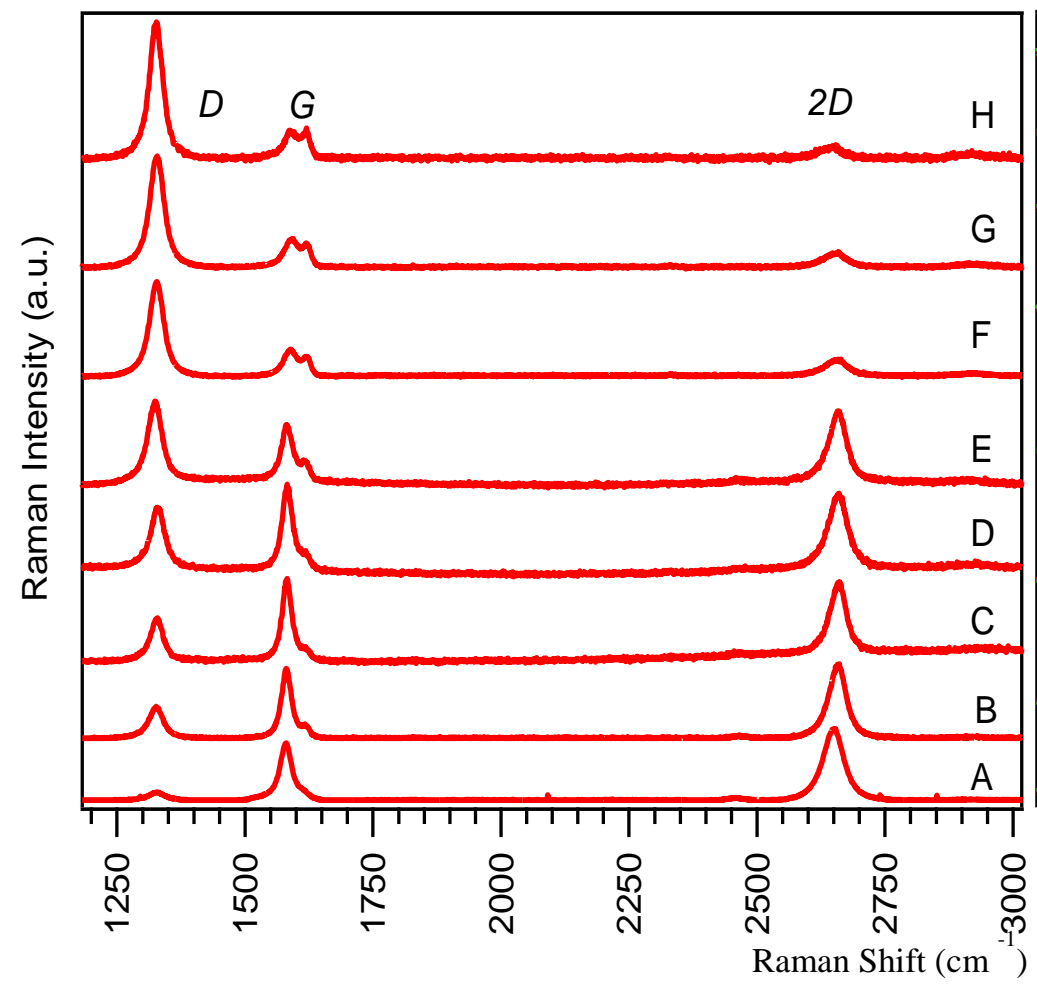

(b)

(c)

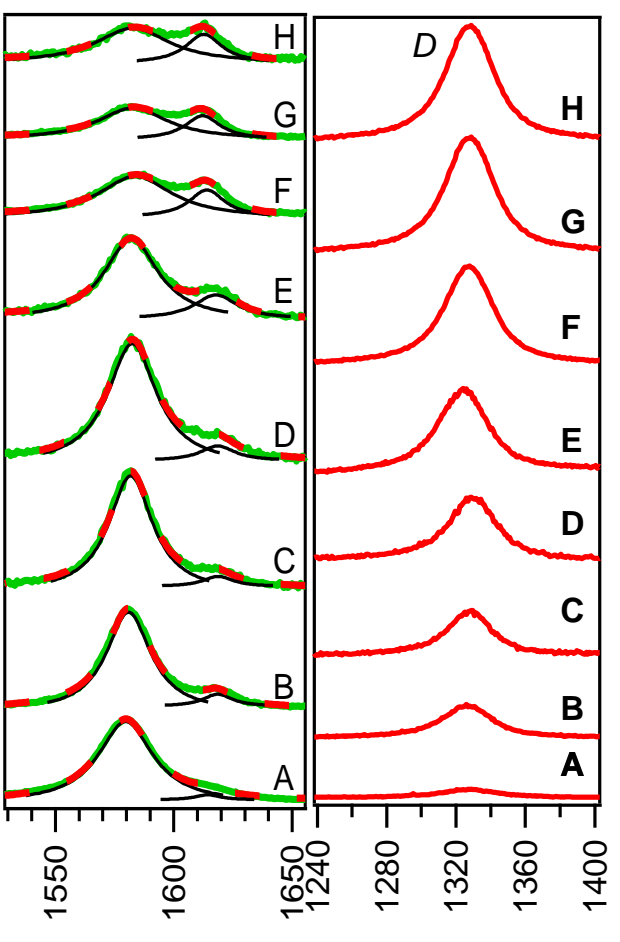

Figure 3.22: (a) Raman spectra of progressively hydrogenated Graphene. (b)

Deconvolution of the $\mathrm{G}$ and $\mathrm{D}^{\prime}$ bands. (c) Evolution of the $\mathrm{D}$ band 
Now let's consider structural changes of Graphene as a result of sequential hydrogenation. Figure 3.22, shows a series of Raman spectra for hydrogenated Graphene including pristine Graphene. The two intense peaks, $G$ band at $\sim 1580 \mathrm{~cm}^{-1}$ and $2 \mathrm{D}$ band at $\sim 2700 \mathrm{~cm}^{-1}$ are characteristic of Graphene samples due to the in-plane vibrational $\left(\mathrm{E}_{2 \mathrm{~g}}\right)$ mode and the two phonon intervalley double resonance scattering, respectively[153-155]. The $2 D$ band of one-layer Graphene is sharp and strong. The increase in layer numbers leads to significant broadening of the width and blue-shift of the $2 D$ band, which could function as the fingerprint in distinguishing the $1 \mathrm{LG}$, 2LG, and multilayer Graphene. The peak at $1340 \mathrm{~cm}^{-1}$ is assigned to $D$ band, which is not detected in disorder-free Graphene and requires defects for its activation via an intervalley double resonance Raman process. Commonly the relative intensity of $D$ band can serve as a convenient measure for the amount of defects in Graphene. After hydrogen plasma treatment (10 W, 1 Torr, 1 min), the Raman spectra of the Graphene sample change significantly. As shown in Figure $3.22 \mathrm{~b}$, a new peak at $\sim 1620 \mathrm{~cm}^{-1}$ is observable in all the Raman spectra of the hydrogenated Graphene layers and its intensity increases as the hydrogenation progresses. The peak at $1620 \mathrm{~cm}^{-1}$ is identified as the $D^{\prime}$ band, which takes place via an intravalley double resonance process only in the presence of defects and merges into the $\mathrm{G}$ band as a shoulder. Figure 3.22c, shows enhancement of the intensity of the $D$ band as the hydrogenation progresses. The extremely weak peak near $2920 \mathrm{~cm}^{-1}$ is assigned to $D+G$ band, which is a combination of $D$ and $G$ modes. The observation of $D, D^{\prime}$ and $D+G$ bands indicate that defects were introduced into the Graphene lattice by the hydrogen plasma treatment[155]. As the hydrogenation progresses, the intensities of the $G$ and $2 D$ bands gradually decrease while the intensities of the $D$ and $D^{\prime}$ bands increase. Emergence of the $\mathrm{D}+\mathrm{G}$ band is evident but remains weak. The modifications of the 
Raman features are caused by hydrogenation of Graphene, which results in the formation of $\mathrm{C}-\mathrm{H} \mathrm{sp} \mathrm{sp}^{3}$ bonds as well as the breaking of the translational symmetry of $\mathrm{C}=\mathrm{C} \mathrm{sp}{ }^{2}$ bonds.

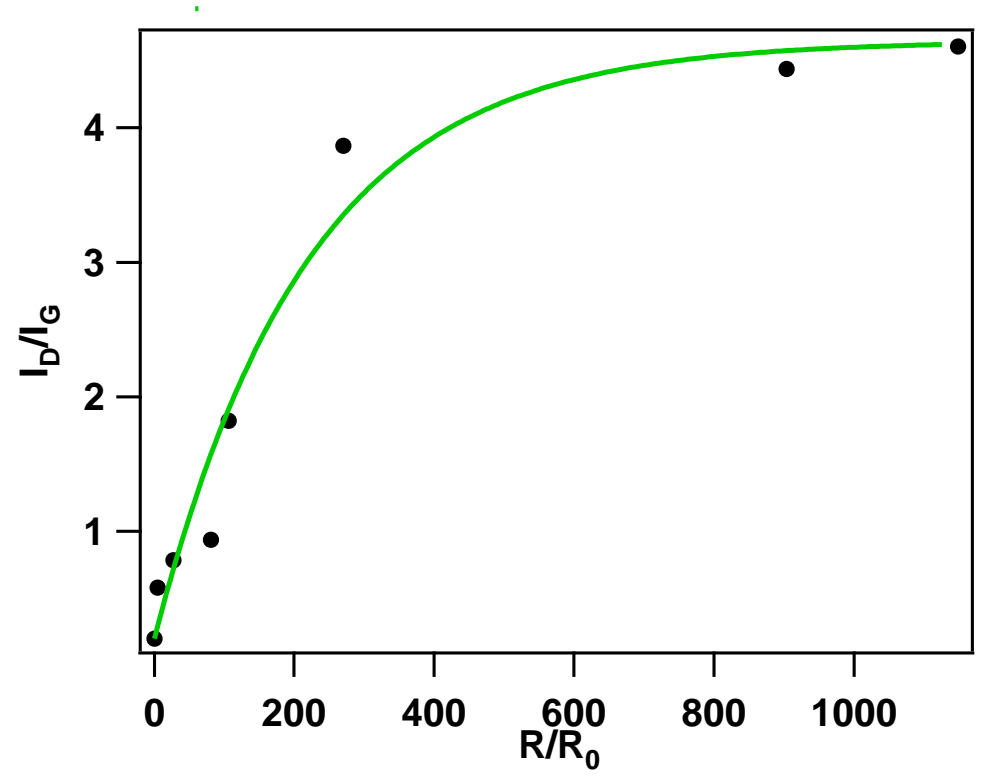

Figure 3.23: Ratio of intensities of $\mathrm{D}$ and $\mathrm{G}$ bands for each hydrogenation as represented by the increase in the room temperature resistance.

Figure 3.23 shows the ratio of the Raman $D$-peak to G-peak intensities $\left(\mathrm{I}_{\mathrm{D}} / \mathrm{I}_{\mathrm{G}}\right)$ for each hydrogenation process characterized by the change in the room temperature resistance. The $\mathrm{I}_{\mathrm{D}} / \mathrm{I}_{\mathrm{G}}$ ratio can be used to estimate the size of defect free areas in the Graphene film. As can be seen in Figure $5 d$ during the initial hydrogenation, $\mathrm{I}_{\mathrm{D}} / \mathrm{I}_{\mathrm{G}}$ ratio increases steeply, but the rate of change becomes slower for heavy hydrogenation indicative of reaching saturation. 

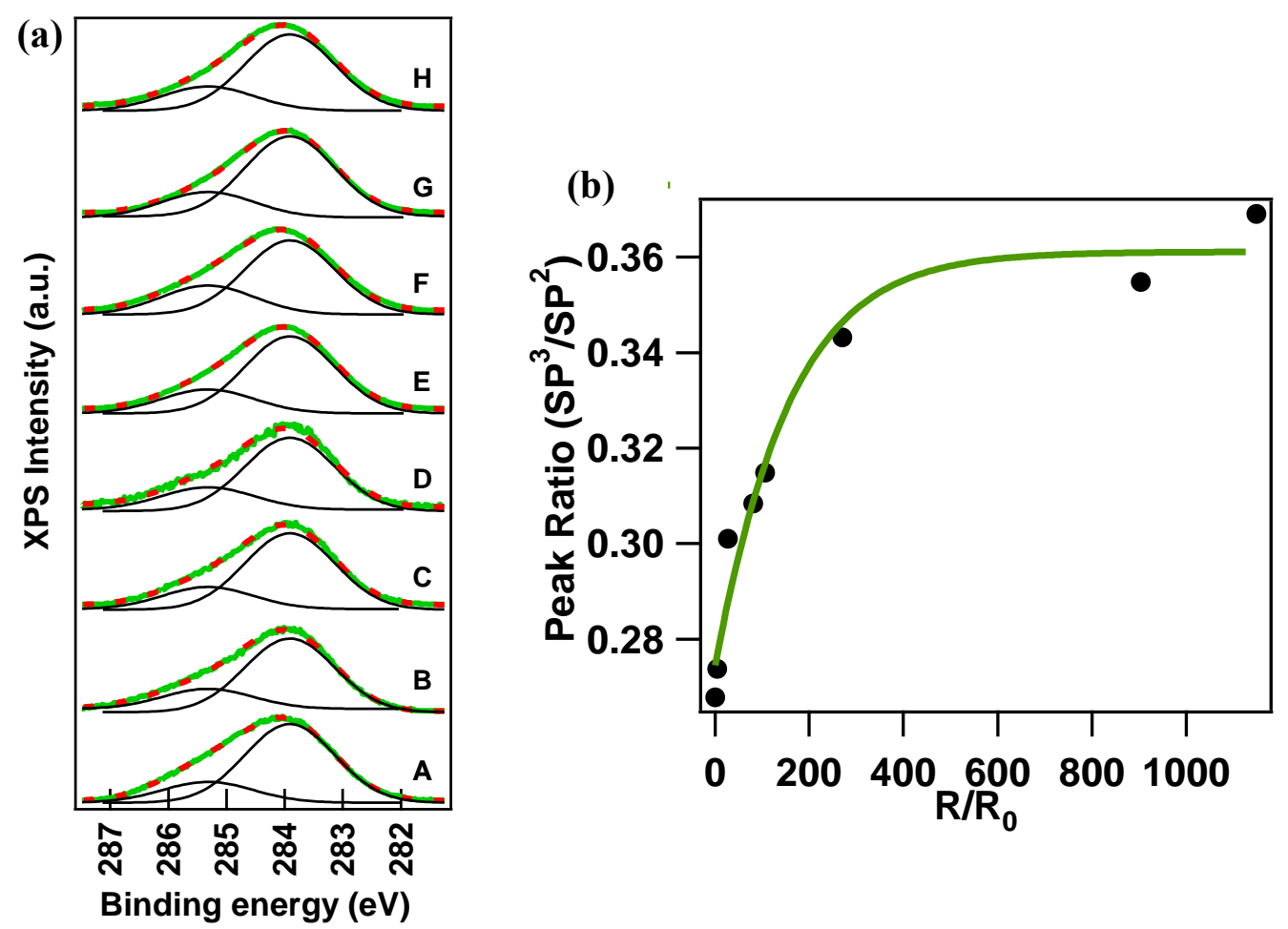

Figure 3.24: (a) XPS results for C1S peak of progressively hydrogenated Graphene (b) Ratio of the intensities for $\mathrm{sp}^{2}$ and $\mathrm{sp}^{3}$ peaks corresponding to each hydrogenation process.

Figure 3.24a shows the evolution of $\mathrm{C} 1 \mathrm{~s}$ spectra fitted with two components. Main peak at a binding energy (BE) of $284.4 \mathrm{eV}$ is assigned to $\mathrm{sp}^{2}$ hybridized $\mathrm{C}$ atoms in Graphene. another peak at higher $\mathrm{BE}$ of $285.05 \mathrm{eV}$ is assigned to $\mathrm{sp}^{3}$ hybridized $\mathrm{C}$ atoms due to the formation of $\mathrm{C}-\mathrm{H}$ and $\mathrm{C}-\mathrm{C}$ bonds by hydrogenation[156]. The intensity ratio of $\mathrm{sp}^{3}$ and $\mathrm{sp}^{2}$ hybridized carbon components, $\mathrm{sp}^{3} / \mathrm{sp}^{2}$ for each hydrogenation step were estimated. This believed to be an indication of the degree of disorder of the $\mathrm{sp}^{2}$ carbon network. As evidenced by Figure $3.24 \mathrm{~b}$ for dilute hydrogenation at the early stages, $\mathrm{sp}^{3} / \mathrm{sp}^{2}$ ratio increases rapidly, but slows down for heavy hydrogenation again indicative of approaching the saturation consistent with the Raman results. 
Metal-insulator transition of graphene can be seen by introducing disorders to metallic behaving samples. Diffusion is the dominant mechanism of the electron conduction in the metallic side which leads to weak localization. Where as, in the insulating side the conduction is via variable range hopping process such as the Mott hopping and the Efros-Shklovskii hopping. In these cases the conductance is exponentially dependent on temperature. With controlled functionalization, one can achive the critical regime of in between the metal-insulator transition with an intermediate level of disorders. For the case of graphene, the Dirac fermions tend to be delocalized against disordering. This yields a Kosterlitz-Thouless-type of critical behavior in the presence of strong disordering[157].

These might account for our observations of the logarithmic or power laws of conductance in disordered graphene. It indicates that the electrons switch from weak localization to strong localization with decreasing temperature. 


\section{CHAPTER 04}

\section{FLUORINE FUNCTIONALIZATION OF GRAPHENE: EVIDENCE OF A BAND GAP}

\subsection{Introduction}

Due to its extraordinary properties graphene continues to attract intense interest in both scientific and industrial communities. The surface of graphene has been functionalized with various derivatives by both covalent and non-covalent means. In graphene oxide, oxygen species are randomly attached to the graphene lattice by breaking the $\mathrm{C}-\mathrm{C} \pi$ - and $\sigma$-bonds which results in a breakdown of conductivity. As described in chapter 03 hydrogenation offers a more controlled means of attaching hydrogen species to the $\mathrm{sp}^{2}$ carbon atoms at least on one side of graphene by breaking the $\mathrm{C}-\mathrm{C} \pi$ bonds. This allows preserving the crystalline order of the carbon lattice, but leads to re-hybridization of the carbon atoms from a $\mathrm{sp}^{2}$ to a distorted $\mathrm{sp}^{3}$ state, which is expected to induce the formation of a band gap. However, hydrogenated graphene is known to lose Hydrogen at moderate temperatures thereby limiting its applications where high temperature stability is required. In order to make more stable graphene derivatives the functional group of interest needs to bind with graphene's carbon stronger than hydrogen. On the other hand, fluorine has higher binding energy to carbon and higher desorption energy than hydrogen. Therefore fluorination of graphene offers more stable surface functionalized graphene derivative compared to graphane. 
Graphene covered with fluorine could be a good option because it is a 2D analogue of the well-known materials: Teflon, which is composed of fluorinated 1D carbon chains, and graphite fluoride (GrF), a multi-layer graphene fluoride.

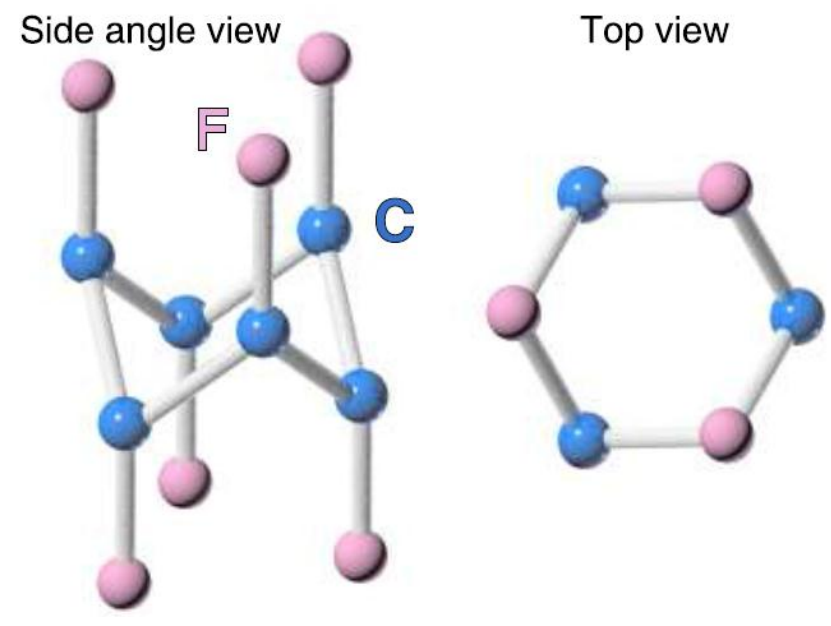

Figure 4.1: Structure of fluorinated graphene[158]

\subsection{Fluorination of Graphene}

Fluorinated graphene is thermodynamically stable in direct contrast to hydrogenated graphene and bulk fluorinated graphite. Several distinct forms of graphene fluoride have been produced recently, which we characterized by their fluorine concentration and atomic configuration.

One type of fluorinated graphene is characterized by an extremely low concentration of fluorine. This introduces p-type doping into the graphene sheet and shown an unexpected colossal negative magnetoresistance effect. Significant reduction in resistance observed in this study under magnetic fields of 9T[159].

In covalent form of substrate supported fluorinated graphene where fluorine is only confined to a single side is due to the presence of the substrate. Substrate supported graphene fluoride is analogous to planar sheet graphite fluoride. Both these 
materials saturate in the same form of $\mathrm{C}_{4} \mathrm{~F}$ under typical fluorination conditions. Single sided graphene fluoride is six orders of magnitude more resistive than graphene[160]. graphene fluoride has being produced by the mechanical exfoliation of planar sheet graphite fluoride[161]. This material approximates single sided graphene fluoride. But this mechanically exfoliated graphene is not strictly single sided indeed. Previous studies suggest that this material is an alternating orientation of the Rüdorff structure, although this hypothesis remains untested[161].

\subsection{In-situ study of controlled fluorination of graphene}

Large-area CVD-grown Graphene was used in this study as discussed in Chapter 3 for hydrogenation. No micro-fabrication processes were involved during the sample preparation[162]. The Graphene specimens (monolayers) utilized in this work were synthesized by the chemical vapor deposition (CVD) method at low pressure $(\sim 100$ mT) on polycrystalline $\mathrm{Cu}$ foils and then transferred onto $\mathrm{SiO}_{2} / \mathrm{Si}$ substrates $[163$, 164], as described in chapter 03 . A custom-designed split ring capacitively coupled RF plasma system (13.56 MHz, Max. power $600 \mathrm{~W}$ ) was used at room temperature using $\mathrm{CF}_{4}$ gas. Transport measurements were done on the pristine graphene sample before functionalizing with fluorine. The thermoelectric power and resistance of Graphene were measured over a temperature range of $300 \mathrm{~K}$ to $550 \mathrm{~K}$. For low temperature measurements, a chip carrier supporting the Graphene sample was transferred to a closed cycled refrigerator (Janis Research Co. CCS-350ST-H) which can be cooled down to a base temperature of $\sim 10 \mathrm{~K}$. Also the sample-containing refrigerator column is enclosed by an electromagnet (LakeShore Model EM4-CV 4inch gap, Horizontal Field) capable of producing magnetic field which can be swept between -1 and +1 Tesla as in figure 3.7. $\mu$ was measured in Hall probe configuration by applying a perpendicular magnetic field. Hall voltage, $V_{H}$ was measured under +1 , 
0 , and - 1 Tesla magnetic fields and corrections were made for parasitic voltages by averaging.

Transport measurements of the pristine graphene were done to understand the fluorination process. Then the sample was transferred to the functionalization chamber. This fluorination process will drive the graphene sample gradually through metal-insulator transition while opening a band gap. Sample was sequentially fluorinated in a highly controllable manner for different fluorine concentrations. This functionalization process was carried out while measuring transport properties in-situ. This enables to monitor the changes in sample properties while sample is still inside the chamber. Figure 4.2 shows plasma functionalization setup. This setup consists of controlled gas delivery system, reaction area, gas removal system etc. $\mathrm{CF}_{4}$ gas is supplied to the system at $5 \mathrm{sccm}$ flow rate and chamber pressure is maintained at 10torr.

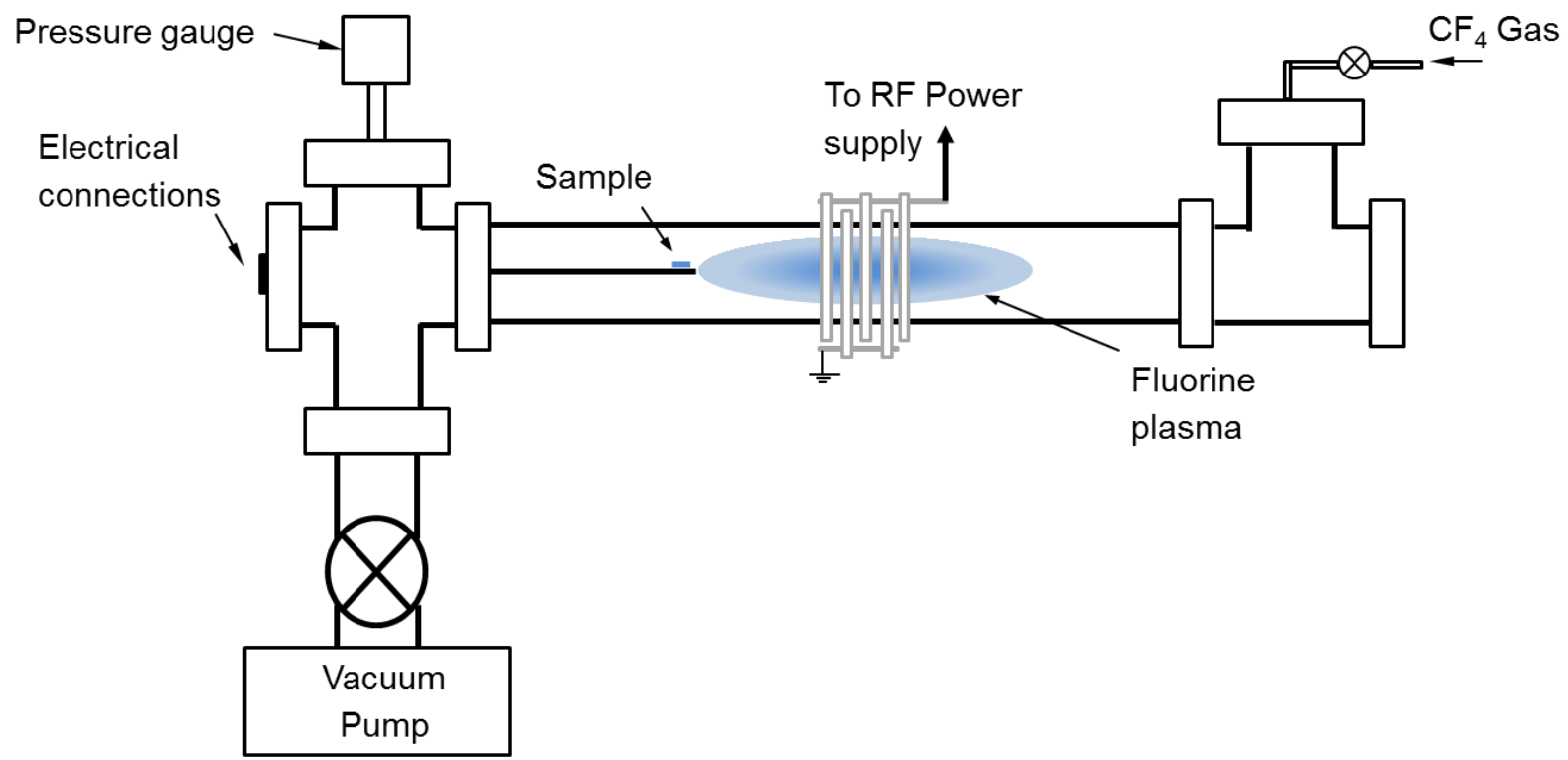

Figure 4.2: Schematic of the plasma functionalization setup 
Figure 4.2 shows the in-situ time evolution of resistance and thermoelectric power of the degassed Graphene during first hydrogenation process. The Graphene sample was carefully exposed to the fluorine plasma for a short period of time ( $\sim 5$ seconds) and the plasma was then turned off. The monitoring of the change in resistance and thermoelectric power was continued.

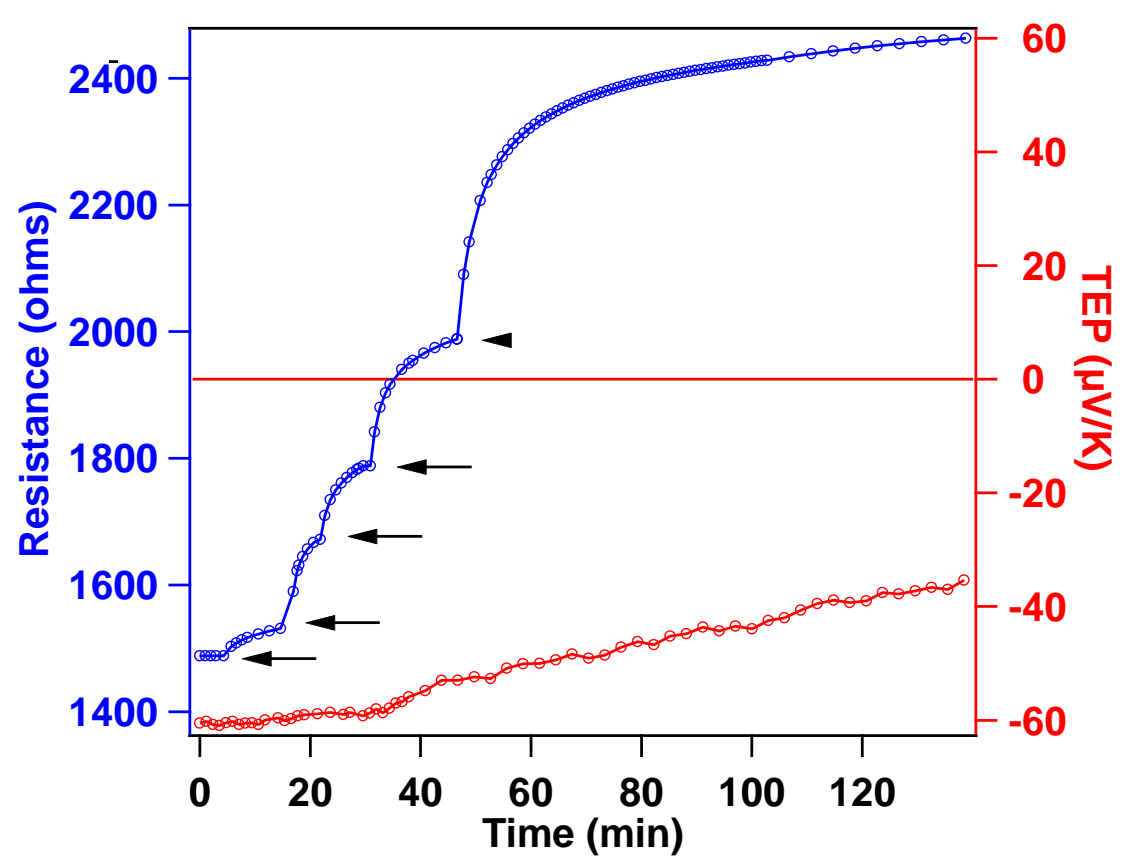

Figure 4.3: In-situ time evolution of the Resistance $R(t)$ and Thermoelectric power $S(t)$ during hydrogenation. The arrow heads represent the initiation of intermittent plasma

The formation of C-F bonding due to the presence of atomic fluorine in the plasma reduces the delocalized electrons (depletion of electrons) in the Graphene network Fermi level will shift towards the Dirac point thereby reducing n-type dopants. This process is clearly shown by the change in the thermoelectric power (Red curve in figure 4.3). Negative thermoelectric power settles at a less negative value after the functionalization process. Extreme care was taken in order not to inflict any damages to the sample during the process. . As shown in Figure 4.3, resistance increased gradually and saturated at a higher $R$ value. Then the plasma was ignited again for $\sim 5$ 
seconds, turned off, and monitored the time evolution of $R$. The intermittent plasma treatments are indicated by the arrows in Figure 4.3. Thermopower, $\mathrm{S}$ was also measured concomitantly. This process was continued until the $R$ approached the desired value. Once the functionalization step is completed, temperature dependence of the transport properties $(R, S, \mu)$ were measured for that particular degree of fluorination.

\section{4 ex-situ functionalization of graphene}

\subsubsection{Low temperature transport measurements}

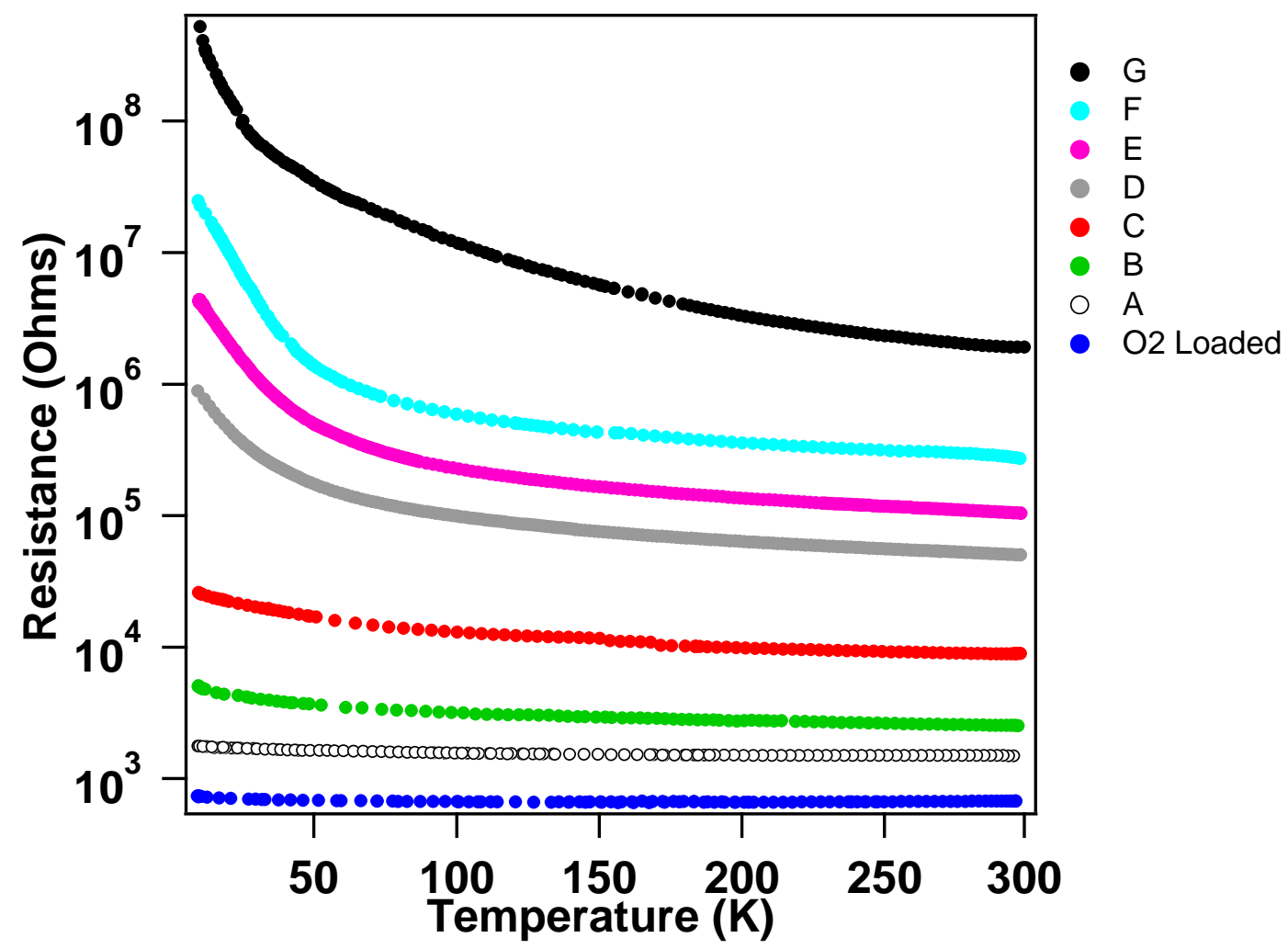

Figure 4.4: Temperature dependence of resistance, $R(T)$ of progressively fluorinated Graphene samples.

Graphene sample was first annealed at 500K under high vacuum conditions $\left(\mathrm{P} \sim 10^{-7}\right.$ Torr). During annealing, $\mathrm{S}$ undergo sign change from $\sim+50 \mu \mathrm{V} / \mathrm{K}$ to $\sim-40$ $\mu \mathrm{V} / \mathrm{K}$. positive $\mathrm{S}$ was an indication of $\mathrm{p}$-type and negative is an indication of $\mathrm{n}$ type 
doping character of the sample. Initially $\mathrm{R}$ was $\sim 700 \Omega$ and gradually increased and reached to a maximum value when $S=0$. Finally $R$ decreased to a saturated value $\sim 1.5$ $\mathrm{k} \Omega[165]$. The ambient p-type behavior has been identified as due to electrochemically mediated charge transfer mechanism between a redox couple in humid air and the Fermi-energy of Graphene[166]. Bottom curve $\left(\mathrm{O}_{2}\right.$ loaded $)$ in Fig 4.4 shows the temperature dependence of $\mathrm{R}$ for the graphene sample before degassing. Temperature dependence of degased sample is shown by curve (A). Controlled plasma fluorination allows to fluorinate Graphene sample to a desired $\mathrm{R}$ value. Curve B through $\mathrm{G}$ shows the temperature dependence of $\mathrm{R}$ during gradual fluorination. Increment of each resistance level is a representation of higher level of fluorination. The process was repeated until resistance was no longer measurable Temperature dependence of the transport properties $(S, \mu)$ were also measured for a particular degree of fluorination.

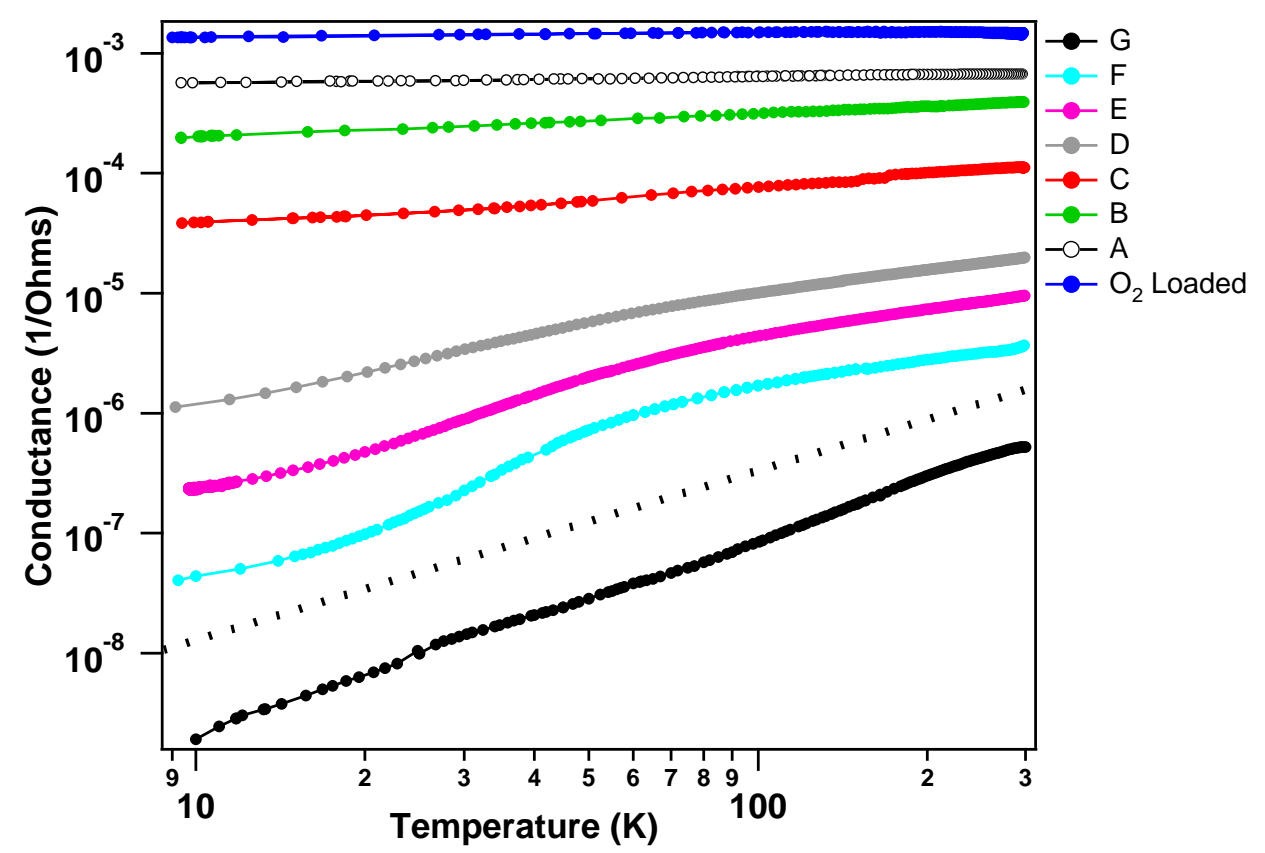

Figure 4.5: Temperature dependence of conductance, $G(T)$ in logarithmic scale for progressively hydrogenated Graphene samples. The dotted line separates metallic samples from insulating. 
Logarithmic dependence of the conductance, $G(\equiv 1 / R)$, vs. the logarithmic temperature is shown in Figure 4.5. This plot helps to separate metallic samples, for which $G$ approaches a constant value at low temperatures, from insulating samples, for which $G$ falls rapidly at low temperatures. Samples with conductivity curves above the dashed line ("A" to " $\mathrm{F}$ ") remain metallic because the conductance remains nonzero for $T \rightarrow 0$, whereas samples with conductance curves below it (curve "G") seem to exhibit insulating behavior for $T \rightarrow 0$. The sample represented by " $\mathrm{G}$ " appears to be close to the transition.

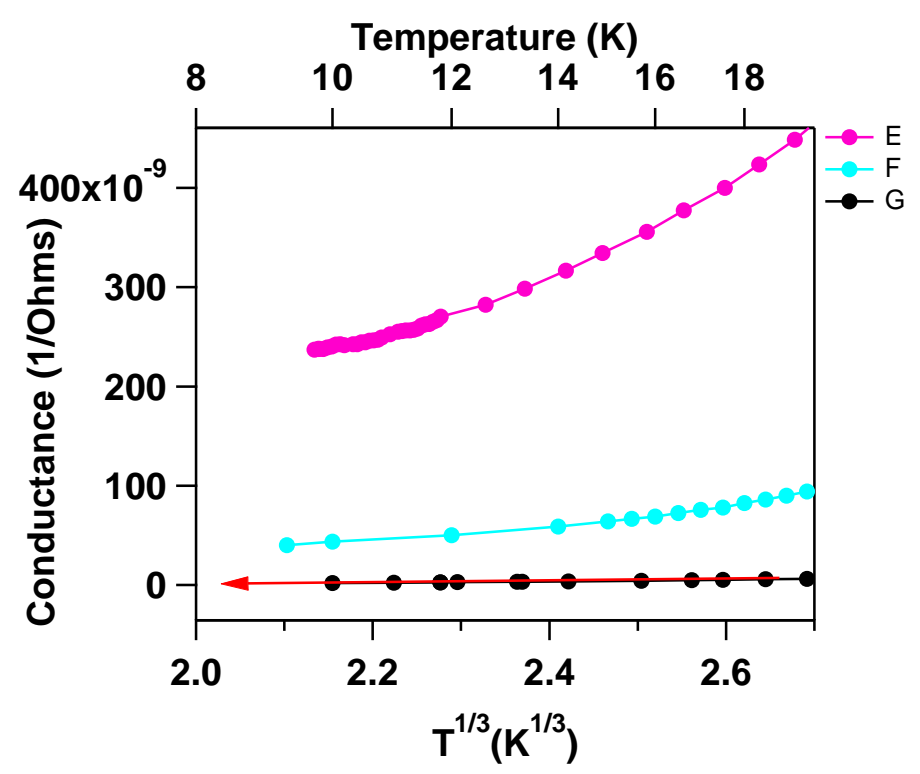

Figure 4.6: Low temperature behavior of the conductivity, $G$ vs $T^{l / 3}$ plot for the three curves E, F and G representing two metallic and critical samples respectively. Notice that the curve $\mathrm{G}$ clearly approaches zero linearly as a function of $\mathrm{T}^{1 / 3}$

Figure 4.6 shows the critical behavior of the conductance. This plot of conductance, $G$ vs $T^{1 / 3}$ in an expanded scale in the vicinity of $G=0$. Closer inspection shows that the data near the MI transition are actually better described by a $T^{1 / 3}$ dependence for low $T$. Conductance for the top two curves ("E and F") remain metallic because the conductance remain nonzero for $T \rightarrow 0$. Conductance for the 
bottom curve (curve "G") varies almost linearly as a function of $T^{1 / 3}$ for temperature below $30 \mathrm{~K}$. this can be confirmed by extrapolating the curve (" $\mathrm{G}$ ") to $\mathrm{T}=0$. Conductance of the bottom curve (curve "G") goes to zero when $T \rightarrow 0$ [167-169].

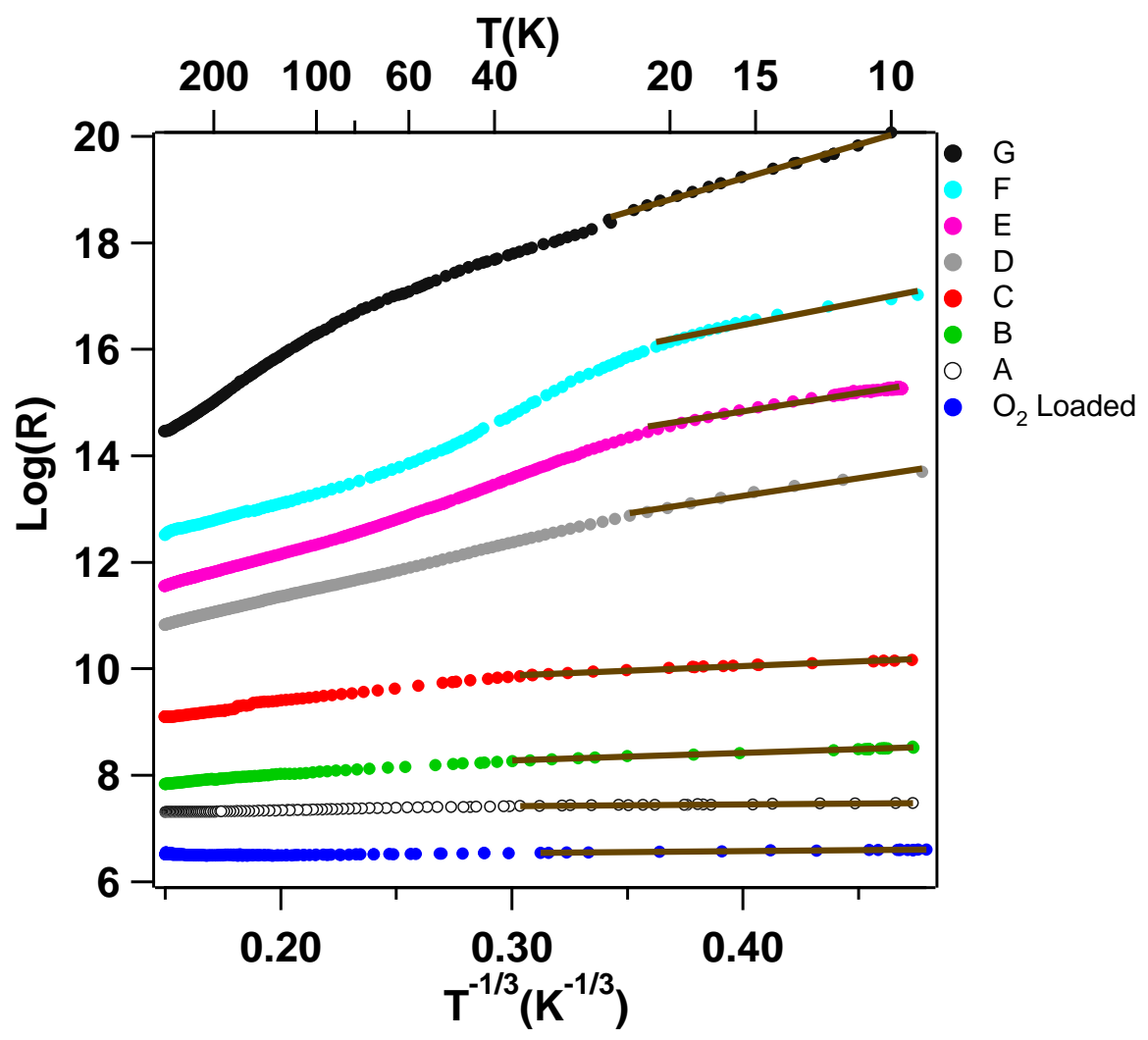

Figure 4.7: At low temperature, the sample resistance is fit to the twodimensional variable-range hopping model

It is known that the temperature dependence of the conductance, $G(T)$ follows the $G(T) \sim \ln (T)$ dependence in the $2 \mathrm{D}$ weak localization (WL) which could be applicable for pristine Graphene.But for hydrogenated Graphene, electrical transport is expected to be governed by hopping conduction. Assuming possibility of the $2 \mathrm{D}$ VRH conduction in our system due to hydrogenation of carbon atoms, we fitted $R(T)$ dependence by classical law for variable range hopping (VRH) $R=R_{0} \operatorname{Exp}\left(\frac{T_{0}}{T}\right)^{1 / 3}$, where $T_{0}=\frac{13.8}{k_{B} N\left(E_{F}\right) \xi^{2}}$ 
$k_{B}$ is the Boltzmann constant, $N\left(E_{F}\right)$ is the density of states at the Fermi energy, $\xi$ is the localization length[170].

In this context we plot Logarithmic $R, \log (\mathrm{R}) \mathrm{vs}^{-1 / 3}$ in Figure 4.7. It is evident from the plot that the data for highly hydrogenated Graphene samples fit very well for the 2-D VRH theory for low temperature regime (below $40 \mathrm{~K}$ ). For higher temperatures, another clear linear range for $\mathrm{T}^{-1 / 3}$ dependence is evident. But for dilute hydrogenation, VRH theory seems to deviate from the experimental data especially for higher temperature.

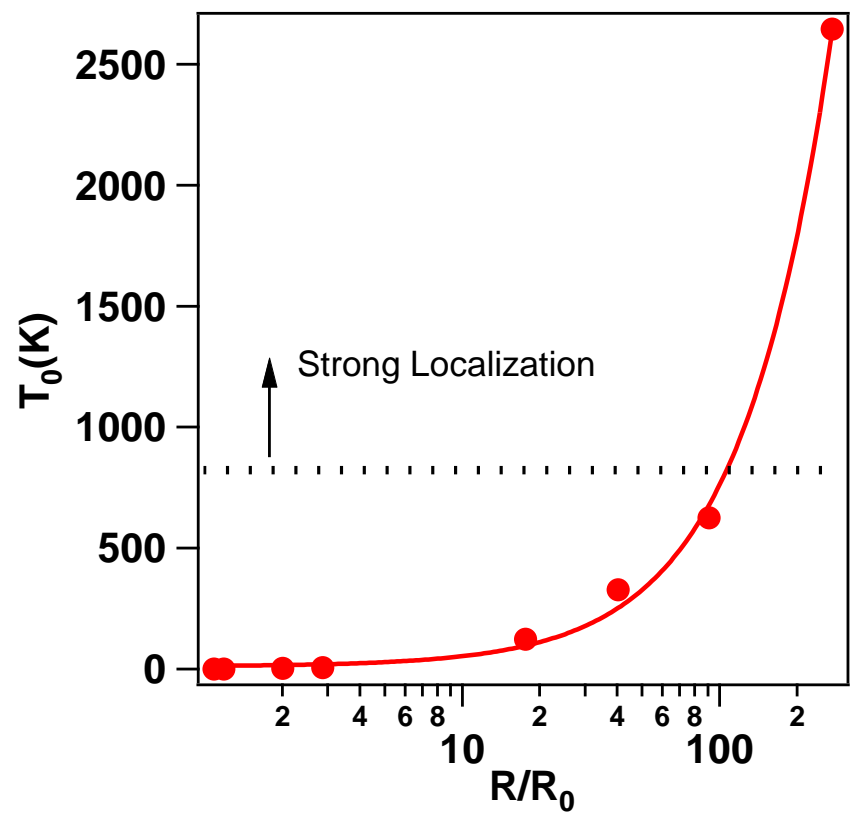

Figure 4.8: $T_{0}$ values (extracted from VRH fitting) for each fluorination process characterized by change in room temperature resistance

Figure 4.8 shows the $T_{0}$ value extracted from VRH analysis at low temperatures as a function of hydrogenation represented by the change in the room temperature resistance, $R / R_{0}$, where $R$ is the room temperature resistances of Graphene after each hydrogenation process and $R_{0}$ is the prior to fluorination (vacuum annealed) resistance. During the first few fluorination processes, $T_{0}$ remains small. 
This implies that VRH is not the dominant mechanism of electron transport for low fluorination. But after the last fluorination process, $T_{0}$ increases five times more as high as $2500 \mathrm{~K}$. Sudden increase of $T_{0}$ marks the transition from $W L$ to $S L$.

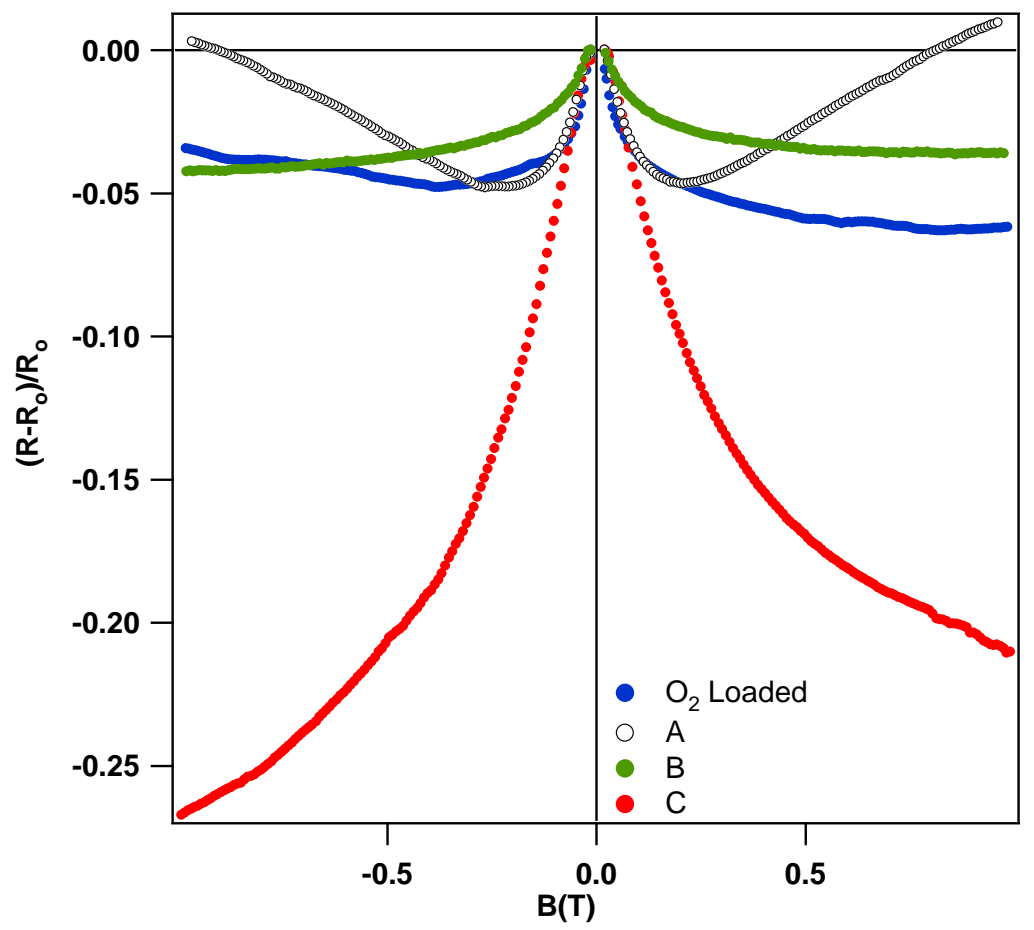

Figure 4.9: Magneto resistance data for progressively fluorinated graphene.

Figure 4.9 shows the magneto resistance data for fluorinated graphene at $10 \mathrm{~K}$. By considering the data shown if figure 4.9, fluorinated graphene systems exhibit a rich variety of magneto resistance. Negative magneto resistance of this magnitude was seen in disordered 2D electron system. The degassed Graphene sample shows low field negative magneto resistance, and governed by classical $B^{2}$ dependence for high magnetic field. There is an asymmetry in the resistance data presumably due to the contact misalignments. This asymmetrical behavior of resistance is not coming from the intrinsic property of hydrogenated graphene. This is resulting from the invasive 
contact geometry. Similar asymmetrical effects have been found for graphene[171]. Magneto resistance has become entirely negative, after first hydrogenation. The negative $M R$ is inherent for the systems where conductivity can be described in the framework of $W L$ theory [171-174]. The low temperature data are analyzed according to $W L$ theory developed for Graphene. The quantum mechanical correction, $\delta \sigma$ to the classical Drude conductivity is given by the equation 3.14. Finally the correction to the magneto resistance is given by equation 3.16 .

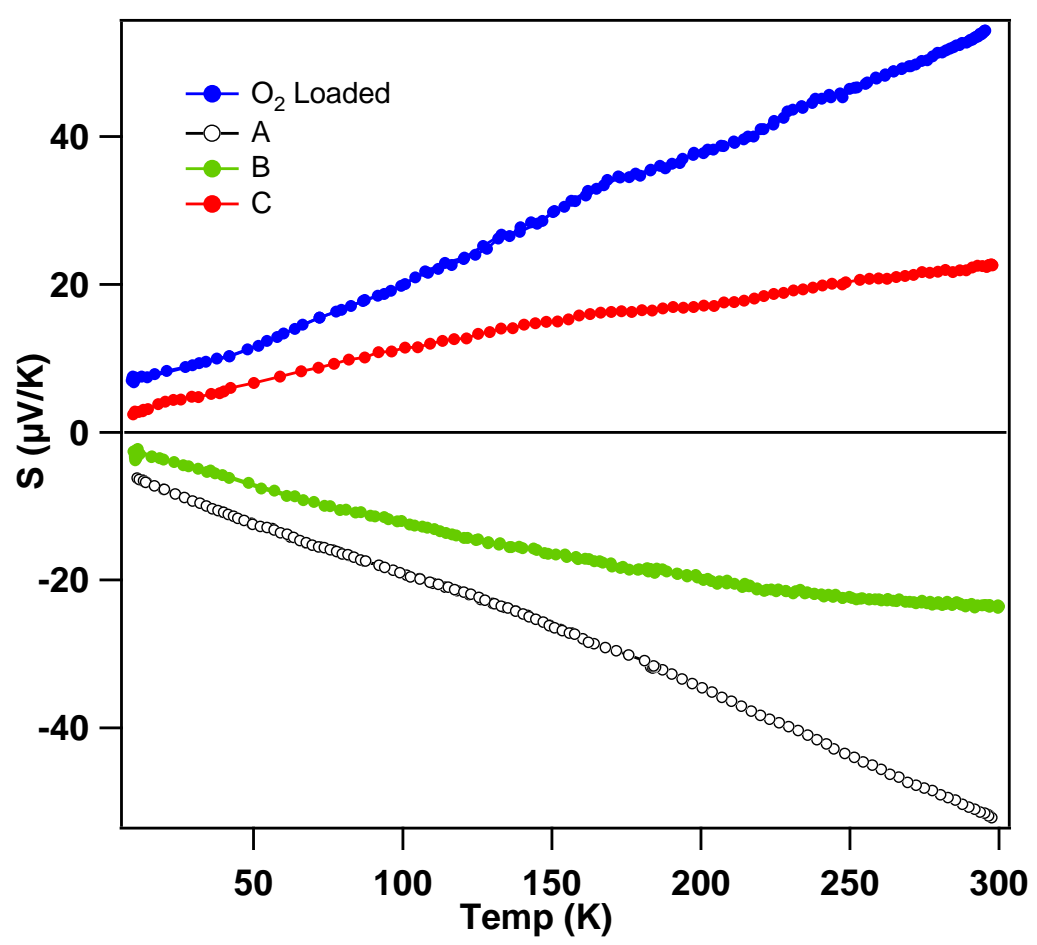

Figure 4.10: Temperature dependence of Thermoelectric power, $S(T)$

Temperature dependence of thermoelectric power for different stages of the fluorination process is shown in figure 4.10. Air exposed CVD grown graphene is known to be doped to p-type. Graphene has become n-type after degassing under vacuum at temperatures $\sim 200{ }^{0} \mathrm{C}$ due to substrate effect. The degassed Graphene sample shows a nearly linear temperature dependence of thermoelectric power over the entire temperature range and remains n-type. After a short fluorination process 
thermoelectric power changed slightly to $\sim-25 \mu \mathrm{V} / \mathrm{K}$. Even after exposure to air at this point, the thermoelectric power remains unchanged. Second fluorination changes thermoelectric power of graphene from negative to positive $\sim+20 \mu \mathrm{V} / \mathrm{K}$. Temperature dependence of thermoelectric power remains p-type almost throughout the entire temperature range with metallic characteristics. Unfortunately the next fluorination caused resistance value high enough to make the thermoelectric power measurements impossible especially at low temperatures. Temperature dependence of both thermoelectric power and resistance showed similar metallic behavior.

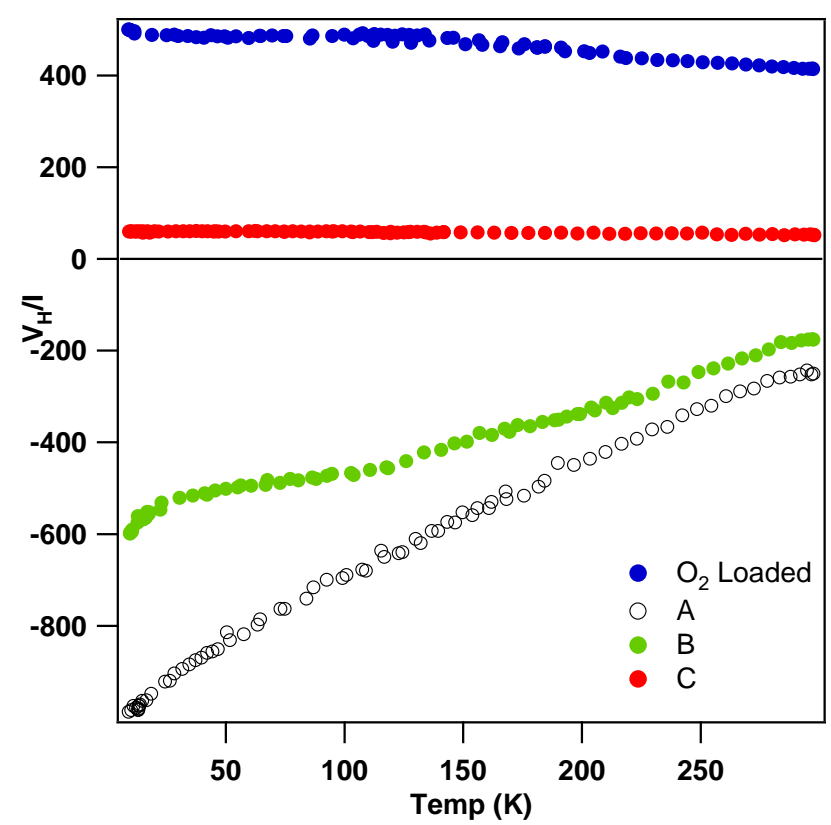

Figure 4.11: Hall voltage over excitation current, $V_{H} / I$ for progressively fluorinated Graphene

Figure 4.11shows the temperature dependence of Hall voltage divided by the excitation current $\left(V_{H} / I\right)$. Graphene before degas shows a $\left(V_{H} / I\right) \sim 420 \Omega$. For the degassed Graphene sample, $\left(V_{H} / I\right) \sim-200 \Omega$ and remains negative in the entire temperature range between $10 \mathrm{~K}$ and $300 \mathrm{~K} . V_{H} / I$ become slightly less negative after the first fluorination $\sim-170 \Omega$ and shows n-type throughout the temperature dependence. After the second fluorination, $\left(V_{H} / I\right)$ increases up to $\sim+50 \Omega$ and the 
temperature dependence of $\left(V_{H} / I\right)$ for this sample remains p-type throughout the entire temperature range. Unfortunately the next fluorination caused $\mathrm{R}$ value high enough to make the $V_{H}$ measurements impossible.

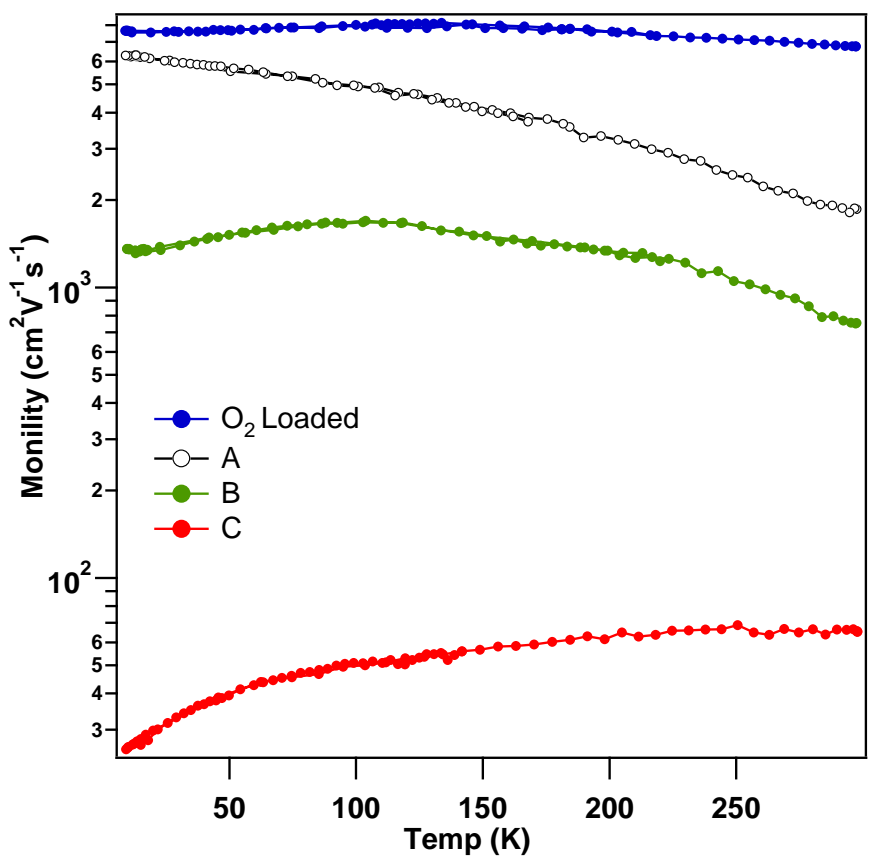

Figure 4.12: Temperature dependence of Hall Mobility $(|\mu|(T))$ for progressively fluorinated Graphene.

Figure 4.12 depicts the temperature dependence of mobility, $\mu(T)$ for the fluorination steps represented by A-C. It can be seen that non-degased sample shows $\mu \sim 6700$ $\mathrm{cm}^{2} \mathrm{~V}^{-1} \mathrm{~s}^{-1}$. This can be due to the effect of $\mathrm{p}$ doping from oxygen and moisture on air. Degased sample shows $\mu \sim 1857 \mathrm{~cm}^{2} \mathrm{~V}^{-1} \mathrm{~s}^{-1}$. Due to the first fluorination, mobility decreased up to $\mu \sim 750 \mathrm{~cm}^{2} \mathrm{~V}^{-1} \mathrm{~s}^{-1}$, and then up to $\mu \sim 65 \mathrm{~cm}^{2} \mathrm{~V}^{-1} \mathrm{~s}^{-1}$ after the second fluorination. 
(a)

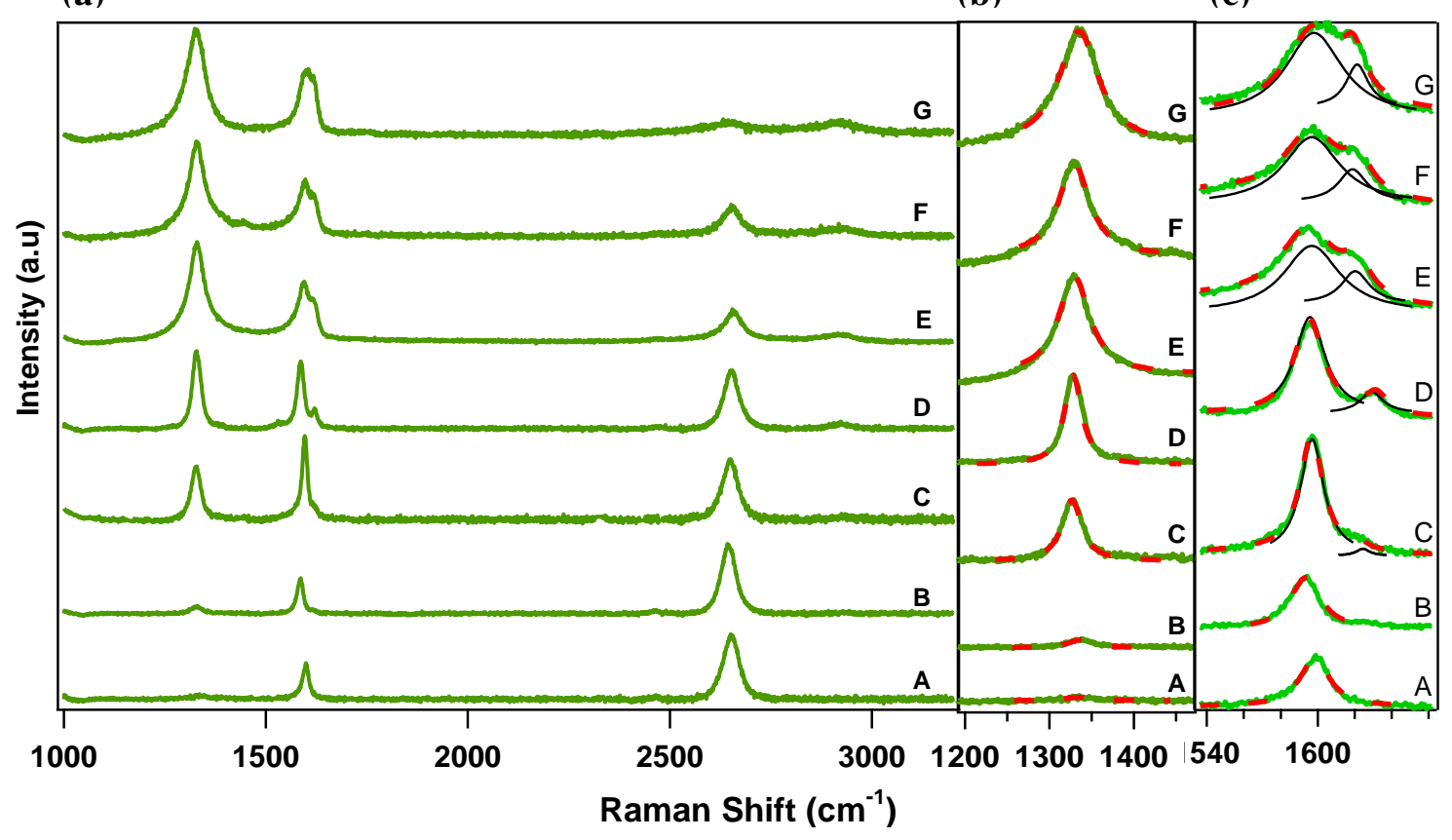

Figure 4.13: Raman spectroscopy results for (a) progressively fluorinated Graphene (b) evolution of the D band (c) Deconvolution of the G and D' bands

Figure 4.13a shows a series of Raman spectra for fluorinated graphene including pristine graphene. The two intense peaks, $\mathrm{G}$ band at $\sim 1580 \mathrm{~cm}^{-1}$ and $2 \mathrm{D}$ band at $\sim 2700 \mathrm{~cm}^{-1}$ are characteristic of Graphene samples due to the in-plane vibrational $\left(E_{2 g}\right)$ mode and the two phonon intervalley double resonance scattering, respectively. The 2D band of pristine mono-layer Graphene is sharp and strong due to the absence of any defects. Broadening and blue-shift of the 2D band could function as the fingerprint in distinguishing the number of layers of Graphene. The peak at $1340 \mathrm{~cm}^{-}$ 1 , which is not detected in disorder-free Graphene is assigned to D band, and requires defects for its activation via an intervalley double resonance Raman process. This D band is serving as a convenient measurement of the amount of disorder in graphene. Due to $\mathrm{CF}_{4}$ plasma treatment, Raman spectra of the Graphene sample change significantly. Figure $6 \mathrm{~b}$ shows a new peak at $1620 \mathrm{~cm}^{-1}$ which is identified as the D' band is observable in Raman spectra of the fluorinated graphene layers and its intensity increases as the fluorination progresses. This new peak appears due to an 
intravalley double resonance process only in the presence of defects and shows up as a shoulder of the $\mathrm{G}$ band. Figure $6 \mathrm{c}$ shows the enhancement of the intensity of the D band as the fluorination progresses. A combination of D and G modes, D+G band starts to appear near $2920 \mathrm{~cm}^{-1}$. The observation of D, D' and D+G bands indicate that defects were introduced into the graphene lattice by the fluorine plasma. During the progressive fluorination, intensities of the $\mathrm{G}$ and $2 \mathrm{D}$ bands gradually decrease while the intensities of the D and D' bands increase.

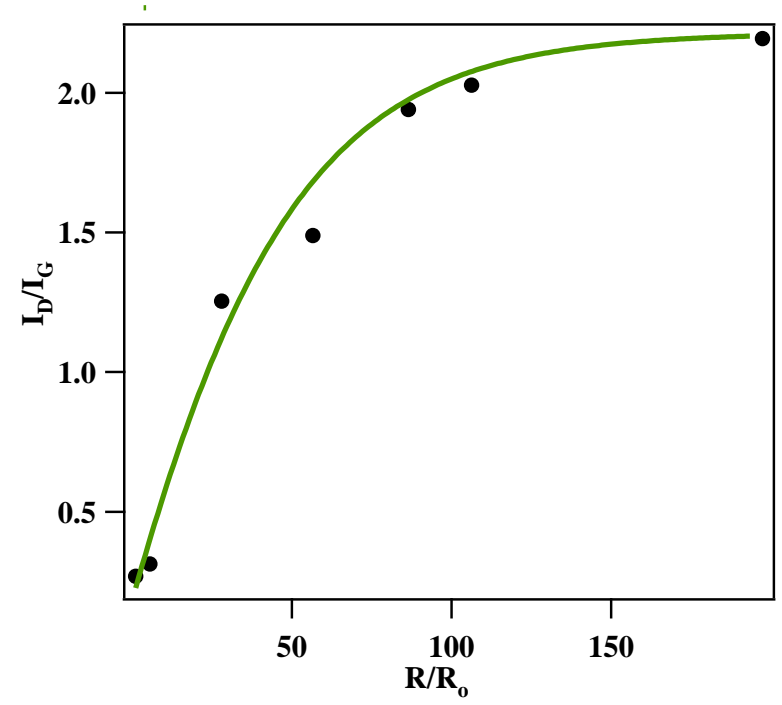

Figure 4.14: Raman spectroscopy results for intensities ratio of D and $\mathrm{G}$ bands for each fluorination as represented by the increase in the room temperature resistance.

Figure 4.14 shows the ratio of the Raman $D$ peak to $G$ peak intensities $\left(I_{D} / I_{G}\right)$ for each fluorination process characterized by the change in the room temperature resistance. The $I_{D} / I_{G}$ ratio can be used to estimate the defects on the Graphene film. The $I_{D} / I_{G}$ ratio increases steeply during the initial fluorination but the rate of change becomes slower for heavy fluorination indicating saturation. 


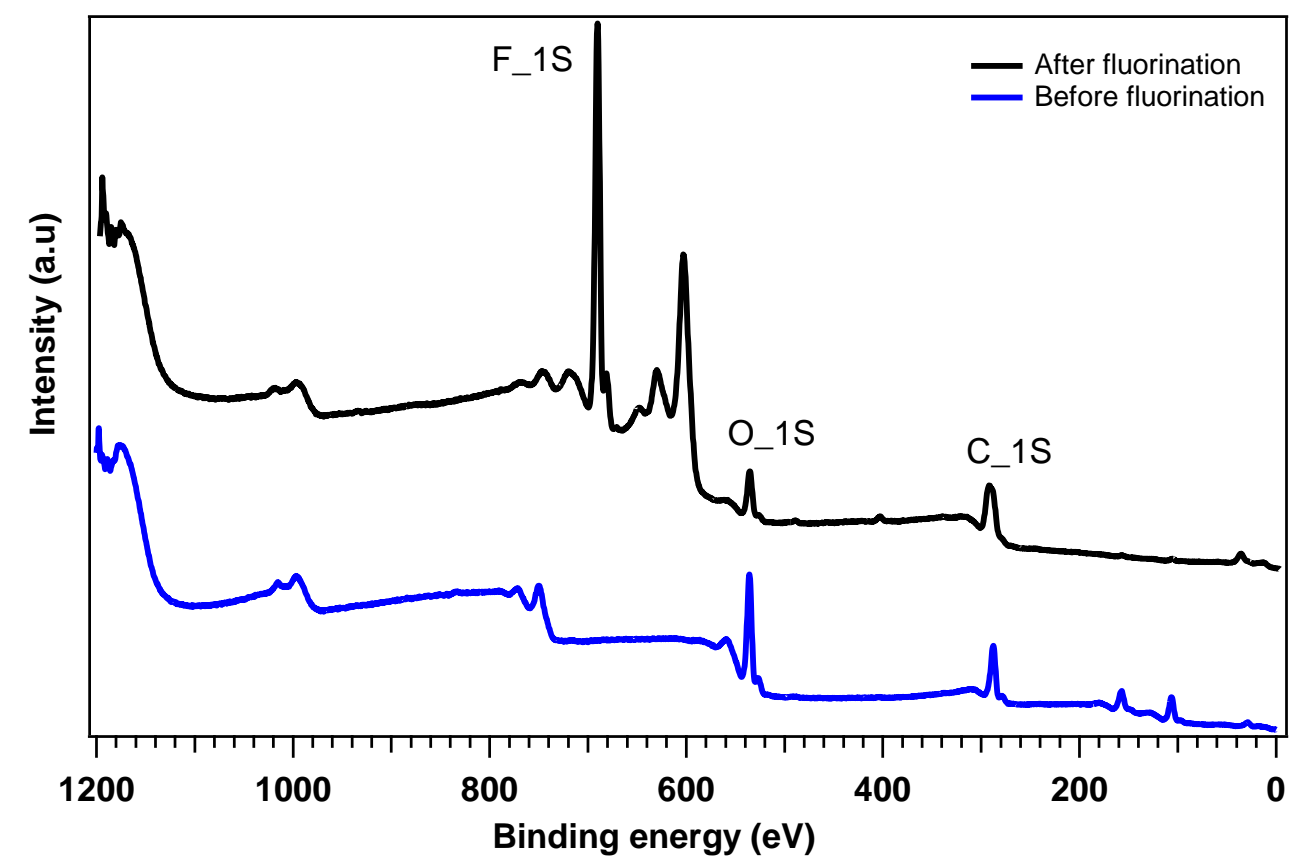

Figure 4.15: XPS survey spectra of progressively fluorinated Graphene

XPS is a well-known technique that has been used as a powerful analytical technique to evaluate compositions of carbon based materials. XPS analysis was, therefore, performed in order to obtain information about the surface composites up to a sample depth of about $10 \mathrm{~nm}$. Figure 4.15 shows the survey XPS spectra of fluorinated graphene. A new peak starts to appear at $687.10 \mathrm{eV}$ on the fluorinated graphene. 


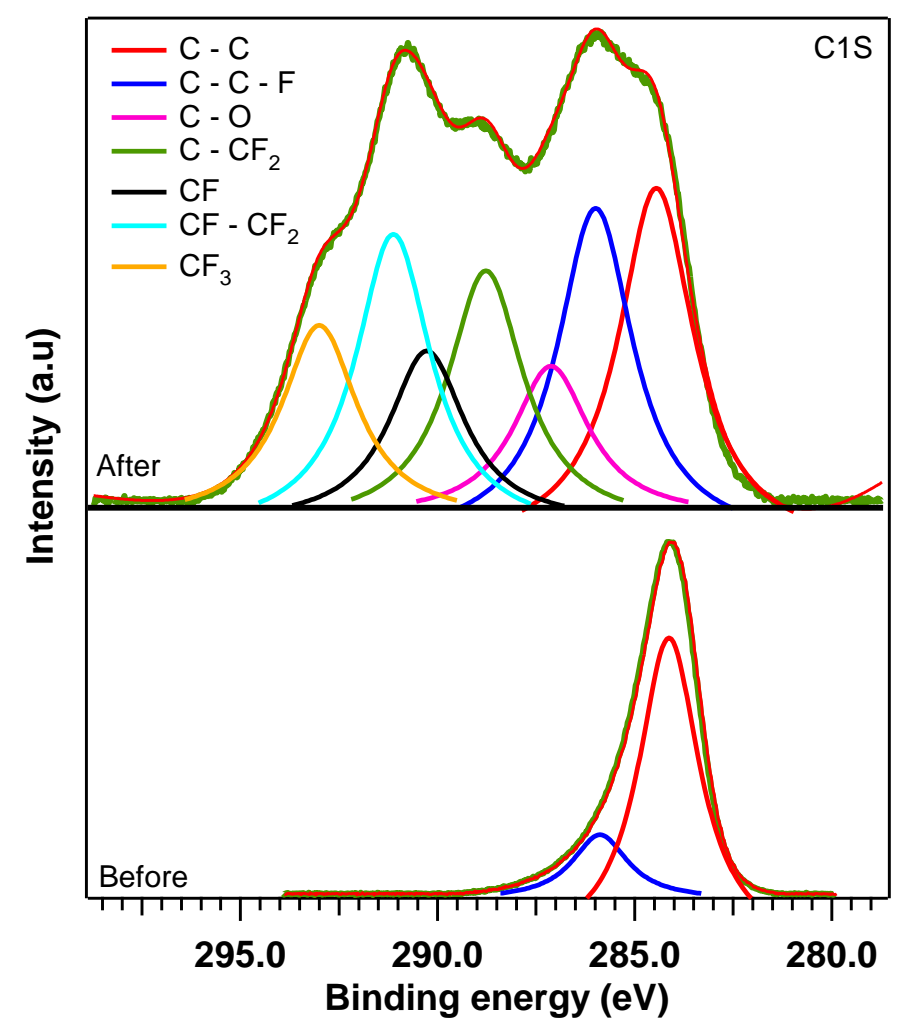

Figure 4.16: XPS results for C1S peak of progressively fluorinated Graphene

Figure 4.16 shows the before and after XPS study done on the fluorinated graphene. C1s spectra fitted with seven components. Main peak at a binding energy (BE) of $284.4 \mathrm{eV}$ is assigned to $\mathrm{sp}^{2}$ hybridized $\mathrm{C}$ atoms in Graphene. Another peak at higher $\mathrm{BE}$ of $285.05 \mathrm{eV}$ is assigned to $\mathrm{sp}^{3}$ hybridized $\mathrm{C}$ atoms due to the formation of $\mathrm{C}-\mathrm{H}$ and C-C bonds by fluorination[175]. Several other peaks will show up in spectrum at 288.2eV for C-F, $289.9 \mathrm{eV}$ for $\mathrm{CF}_{-} \mathrm{CF}_{2}, 285.8 \mathrm{eV}$ for $\mathrm{C}-\mathrm{CF}, 286.9$ for $\mathrm{C}^{-\mathrm{CF}_{2}}, 292.1 \mathrm{eV}$ for $\mathrm{C}-\mathrm{F}_{2}, 293.7 \mathrm{eV}$ for $\mathrm{C}-\mathrm{F}_{3}[176]$. 


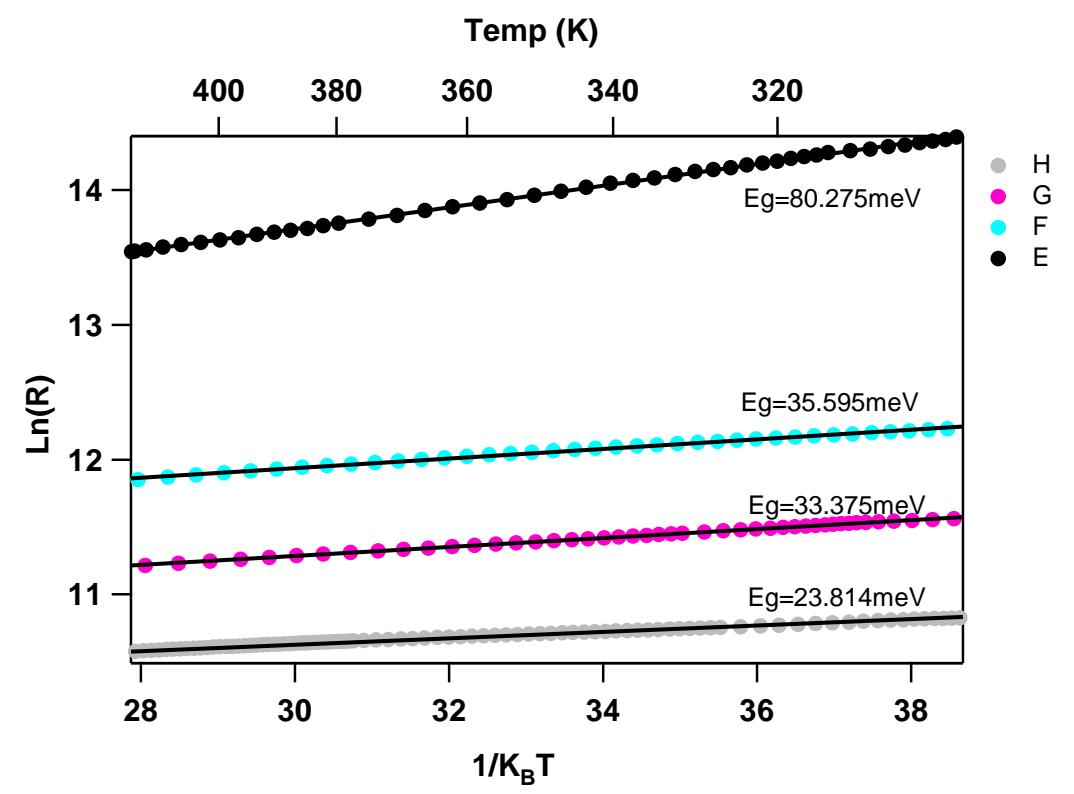

Figure 4.17: The Arrhenius plot of $\log (R)$ vs $\left(1 / k_{B} T\right)$ for densely fluorinated graphene samples at higher temperatures. The slope of the linear range is used to extract the band gap values.

In order to estimate the band gap, the temperature dependance of the four-probe resistance of the fluorinated samples were measured at higher temperatures upto 420

K. The Arrhenius plot of $\operatorname{Ln}(R)$ vs $\left(1 / k_{B} T\right)$ for densely fluorinated graphene samples are shown in Figure 4.17. The band gap, $E_{g}$ was estimated using the temperature dependance of the conductivity, $\sigma=\sigma_{0} \operatorname{Exp}\left(-E_{g} / 2 k_{B} T\right)$, for an intrinsic semiconductor. The highest band gap for the most fluorinated grpahene sample is found to be $\sim 80$ meV which is several orders of magnitude less than what is expected from theoretical predictions of $\sim 3.5 \mathrm{eV}$. A systematic behavior of the band gap value is observed as the fluorination progresses. 


\section{CHAPTER 05}

\section{OPTIMIZATION OF MWNT BASED CF ${ }_{X}$ ELECTRODES FOR PRIMARY AND SECONDARY BATTERIES}

\subsection{Introduction}

Lithium/Carbon fluoride $\left(\mathrm{CF}_{\mathrm{X}}\right)$ primary batteries are very attractive because they offer very high-energy density, long-storage life, very good safety record, a wide temperature operating range, and very low self-discharge[177]. These batteries are found in a wide range of applications in military[178], aerospace[179], electronics[180], and medical industry[180, 181]. This relates to the main purpose of this proposal because if it is possible to increase the energy capacity of these batteries with the choice of a novel and low cost material as the cathode then it would be immensely useful to many fields and would cause a great positive impact on the world and technology. Traditional CFx based cathode materials are formed by hightemperature intercalation of fluorine gas into graphite powder[182] paving the way for high energy density $(250 \mathrm{Wh} / \mathrm{kg}), 7$ year shelf life primary batteries. Tunable Cathode in Li/CFx batteries allows the cell's fundamental properties to be fine-tuned for optimal energy and power density. The Li/CFx cells are especially well-suited for medical applications due to their relatively flat discharge profile, low internal resistance and light weight. Key medical applications include: drug infusion pumps, neurostimulators also known as implanted pulse generator (IPG), pacemakers, bonegrowth stimulators, glucose monitors, defibrillators and other implantable and external devices[183]. Compared to other lithium battery chemistries, Li/CFx typically delivers higher gravimetric energy density, higher volumetric energy 
density, a wider temperature range, exceptional shelf life and optimum price/performance[184]. It is believed that performance characteristics of Li/CFx technology are superior to other primary lithium battery chemistries. Supercapacitors on the other hand are energy storage devices with fast charge and discharge rates, and high power densities and have drawn much attention recently in electric/hybrid vehicles, heavy-construction equipment and grid utility storage[185]. Conventional electrode materials for ECs are activated carbons with nano-porosity and high surface area[186]. Although a lithium anode can be coupled with a variety of different cathode and electrolyte materials, four combinations lead the market today in primary lithium batteries: Lithium/Manganese Dioxide[187], Lithium/Sulfur Dioxide[188], Lithium/Thionyl Chloride[189] and Lithium/Polycarbon Monofluoride[190]. The fact that all these different types of primarylithium batteries continue to exist indicates that each one has some compelling advantage as well as one or more limitations. For example, Sulfur Dioxide and Thionyl Chloride have relatively high energy and power densities, along with wide operating temperature ranges, but suffer from safety and environmental concerns that make then unsuitable for many applications. Manganese Dioxide and Polycarbon Monofluoride are safe and relatively benign environmentally, but fail to pack the power demanded by some applications.

Recent innovations in the formation of carbon fluoride powder hold the potential to eliminate these traditional trade-offs. The advanced Lithium/Carbon Fluoride (Li/CFx) battery maintains the benefits of high energy and power densities, wide operating temperature range and long shelf life found in Sulfur Dioxide and Thionyl Chloride batteries, while employing a solid cathode (with no heavy metals or other toxic materials) to eliminate the safety and environmental concerns. In addition, the advanced CFx battery possesses none of the operational problems such as 
passivation exhibited by some other batteries. Perhaps the most significant advancement found in these new Lithium/Carbon Fluoride batteries is the ability to customize or tune the cathode to meet an application's specific requirements. By altering how fluorine is introduced into the carbon structure at the atomic level during the manufacturing process, the battery's fundamental properties can be changed in ways that favor higher energy or power densities and better rate capability[191].

Recently, it has been reported that subfluorinated $\mathrm{CF}_{x}$ materials where $0.3<x<0.66$ are capable of supporting discharge rates as high as $5 \mathrm{C}$ at room temperature with excellent utilization[192]. Another major advantage of the advanced CFx battery is its ability to exceed all others in both power density and maximum safe current draw. Laboratory tests have demonstrated up to an eight times improvement in high-current applications, and a nearly two times improvement in low-current applications. This makes the advanced CFx battery particularly well-suited for applications that require high sustained or pulse currents. Multiwalled Carbon nanotubes (MWNTs) are candidate material of choice for the use in batteries field due to their unique electrical and mechanical properties, such as the excellent electrical conductivity at room temperature and the high aspect ratio. Despite the success of lithium/carbon fluoride (Li/CFx) on conventional they do not perform well under high rate conditions, exhibit performance loss in low temperature operation and are more costly than competing primary lithium chemistries. Lower cost by using a low temperature fluorination process and selection of MWNTs as the carbon versus conventional usage of coke utilize less fluorine (sub fluorinate) to improve performance and conductivity, including both power and rate capabilities. Also the use multi-walled carbon nanotubes to provide increased surface area to achieve better rate capabilities as well as improve conductivity. Conventional manufacturing of $\mathrm{CF}_{\mathrm{X}}$ cathode material 
involves use of $\mathrm{F}_{2}$ gas at high temperatures. $\mathrm{F} 2$ is highly toxic, corrosive, and can cause ignition of organic material on contact. Another alternative technique utilizes HF acid under ambient condition, but does little to reduce risk since HF is also highly corrosive and dangerous to handle. Moreover, there are a limited number of facilities in the US that manufacture CFx materials. The second approach is to mix the CFxbased materials with other cathode materials such as $\mathrm{MnO}_{2}[193]$, SVO[194] and $\mathrm{MoO}_{3}$ to develop a hybrid structure, but the progress in this area is limited without significant improvement on the rate performance.

\subsection{Fluorine functionalization of MWCNT}

Commercially available NanoBlack ${ }^{\mathrm{TM}}$ Multi-Wall carbon nanotubes were used to prepare battery electrode materials. As reported by the producer, MWCNT have been obtained via Catalytic Assisted Chemical Vapor Deposition (CCVD). Their average outer diameter was of about $10 \mathrm{~nm}$.

(a)

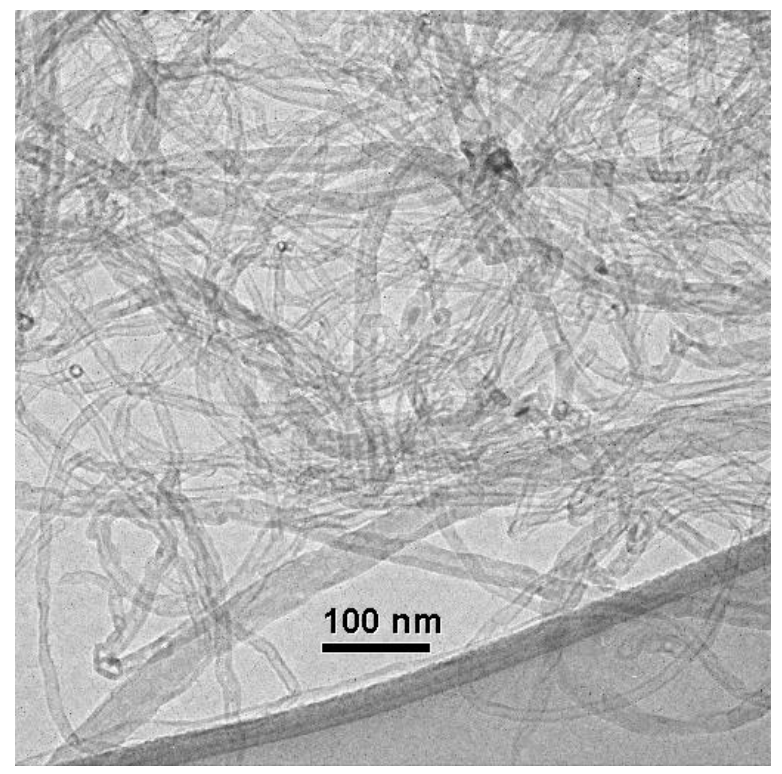

(b)

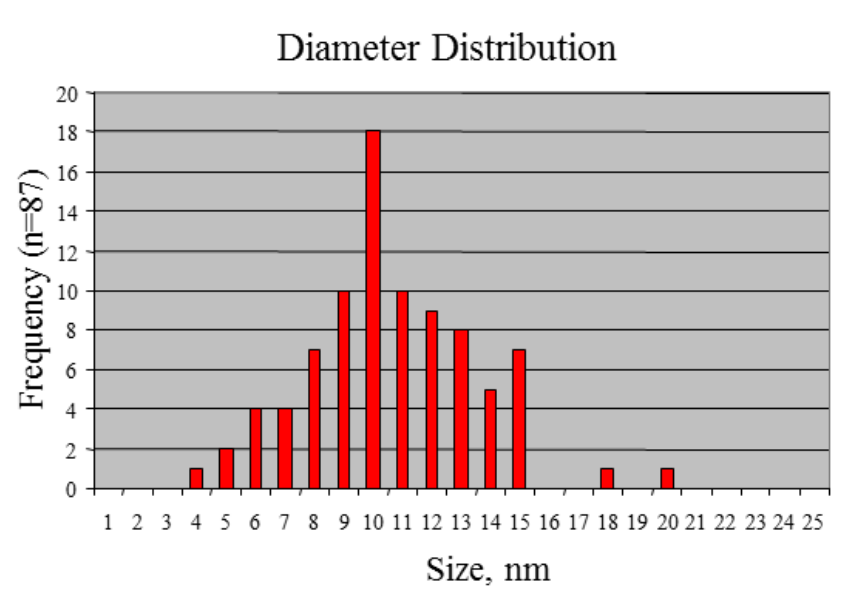

Figure 5.1: (a) TEM image of MWCNT. (b) Diameter distribution of MWCNT. 
A custom-designed, split ring, capacitively coupled RF (radio frequency) plasma system (13.56 MHz, Max. power $600 \mathrm{~W}$ ) was utilized for fluorination of MWNTs. MWNTs were loaded in to a quartz boat and $\mathrm{CF}_{4}$ gas was introduced at controlled flow rates and pressure.

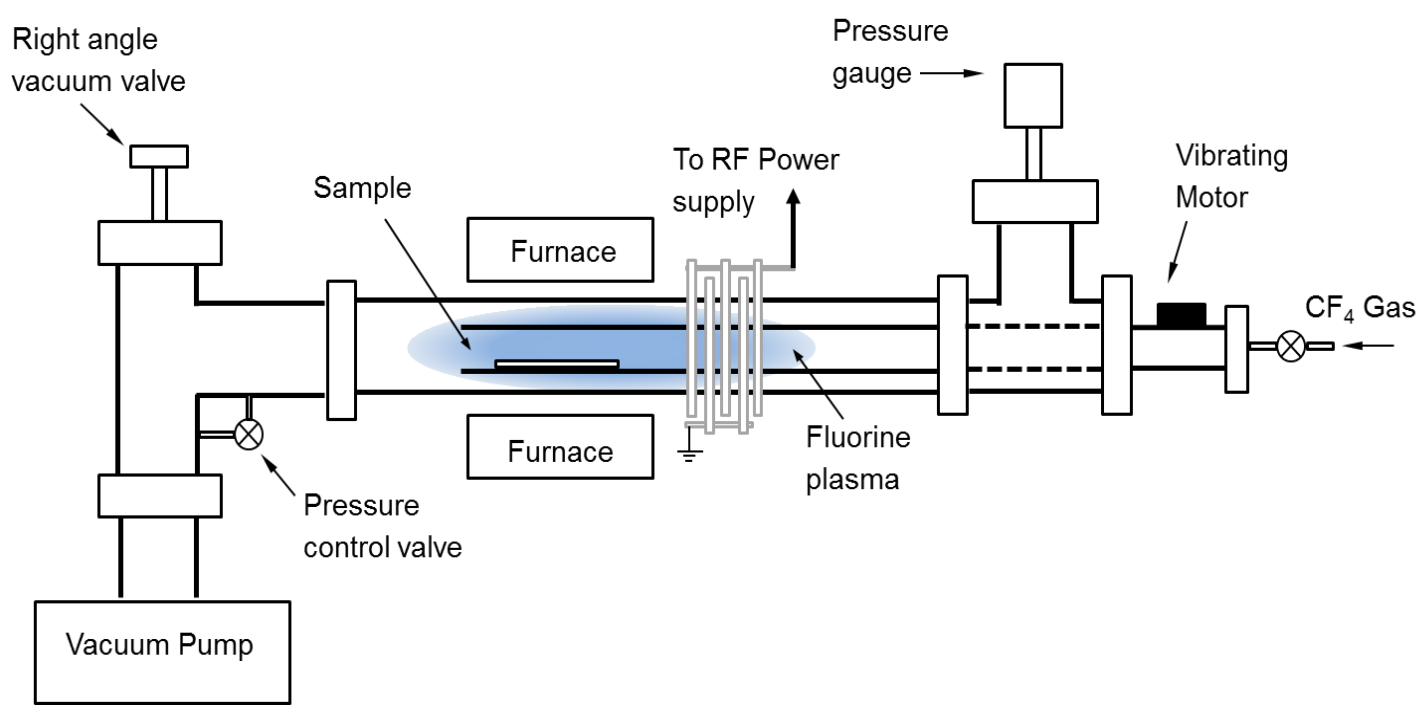

Figure 5.2: Schematic diagram of fluorination chamber

Figure 5.2 shows plasma functionalization setup. $\mathrm{CF}_{4}$ gas is supplied to the system at a $10 \mathrm{sccm}$ flow rate and chamber pressure is maintained at 5 torr. Sample is heated up to $150{ }^{\circ} \mathrm{C}$. This reactor consists of a special dual tube configuration. A vibrator attached to the smaller inner tube enables shaking of the sample holder during the functionalization process. This will facilitates homogeneous fluorination and significantly cuts down the functionalization time. This set up is also equipped with a pressure control valve to regulate the pumping and venting rate of the chamber. Fast pumping and venting can cause loss of powdered material in the sample holder. 


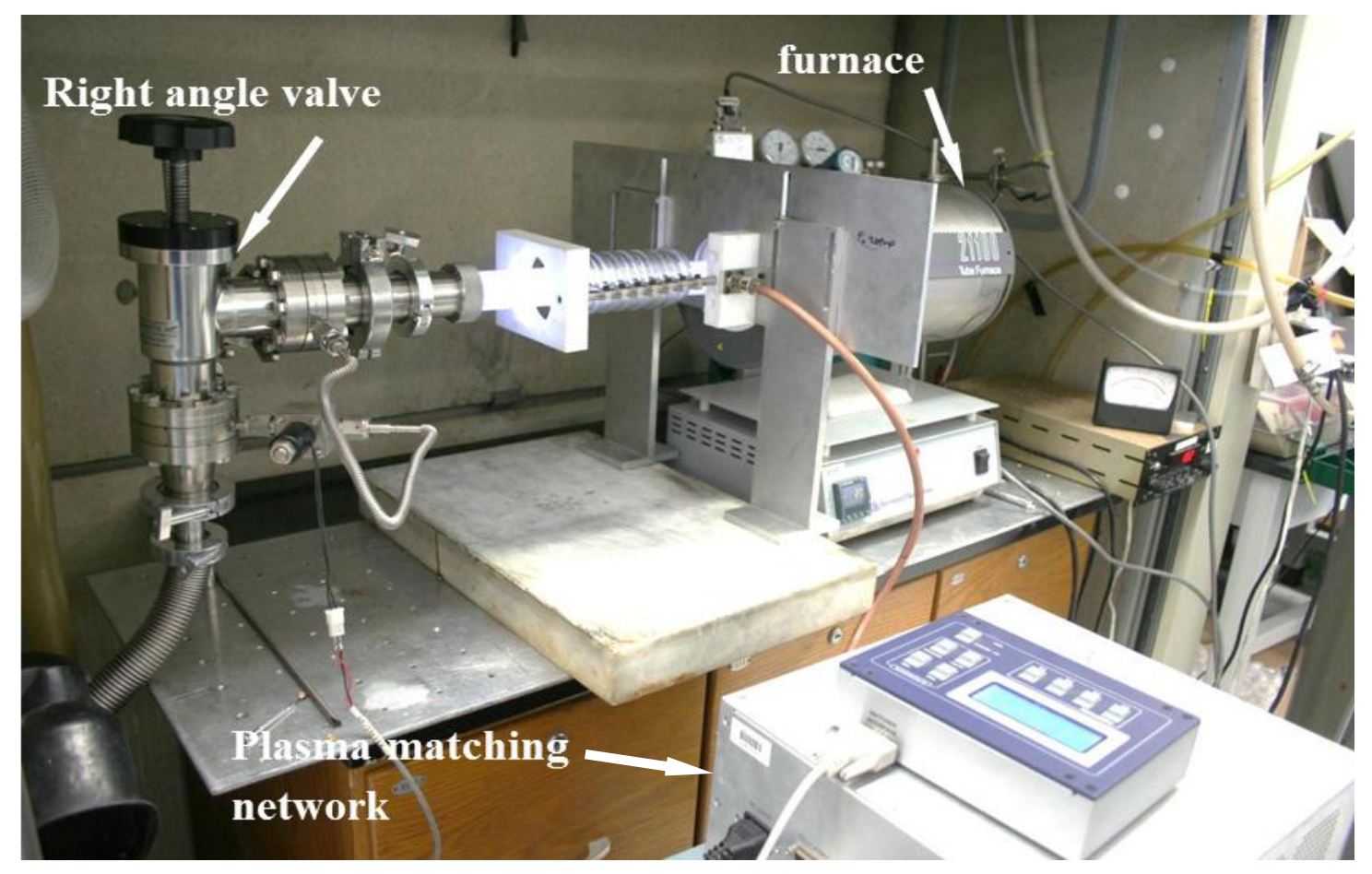

Figure 5.3: Plasma functionalization setup

Figure 5.3 shows a picture of the plasma functionalization setup. $\mathrm{CF}_{4}$ gas is introduced once the sample chamber is slowly pumped to the base pressure. MCNTs of $\sim 100 \mathrm{mg}$ were functionalized for different fluorine concentrations $(2 \mathrm{hr}, 4 \mathrm{hr}, 8 \mathrm{hr}$ and $12 \mathrm{hr})$.

(a)

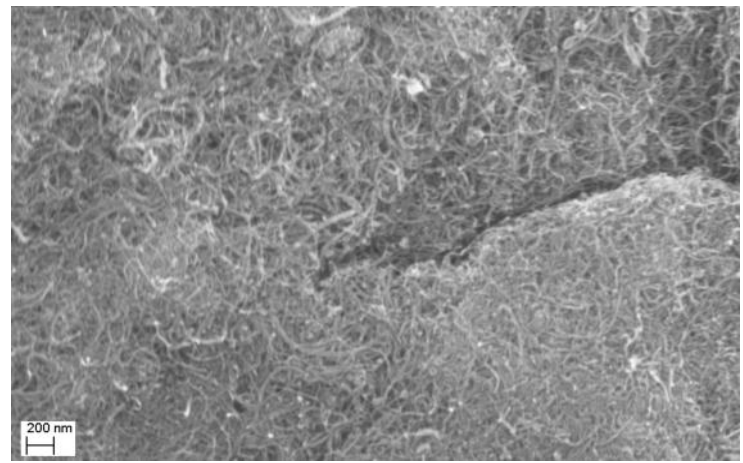

(b)

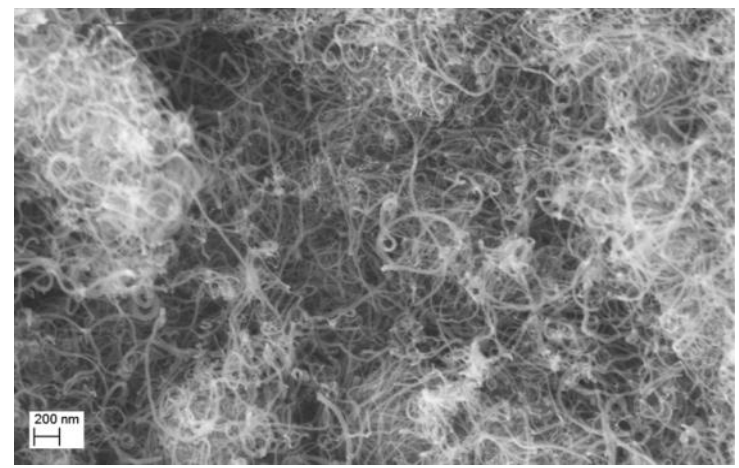

Figure 5.4: SEM image of MWNTs subjected to a controlled mild fluorination ( $2 \mathrm{hr}$ treatment). 
Figure 5.4 shows the SEM images of MWNTs before and after fluorination. It is evident that the MWNTs become larger in diameter and better dispersed as a result of fluorination still manintaining their structural integrity. The Fluorination was characterized using XPS (x-ray photo emission spectroscopy) and EDX (EnergyDispersive X-ray spectroscopy). Theese two methods of finding the fluorine and carbon percentages involve careful analysis of carbon and fluorine specific signatures of each technique.

(a)

(b)

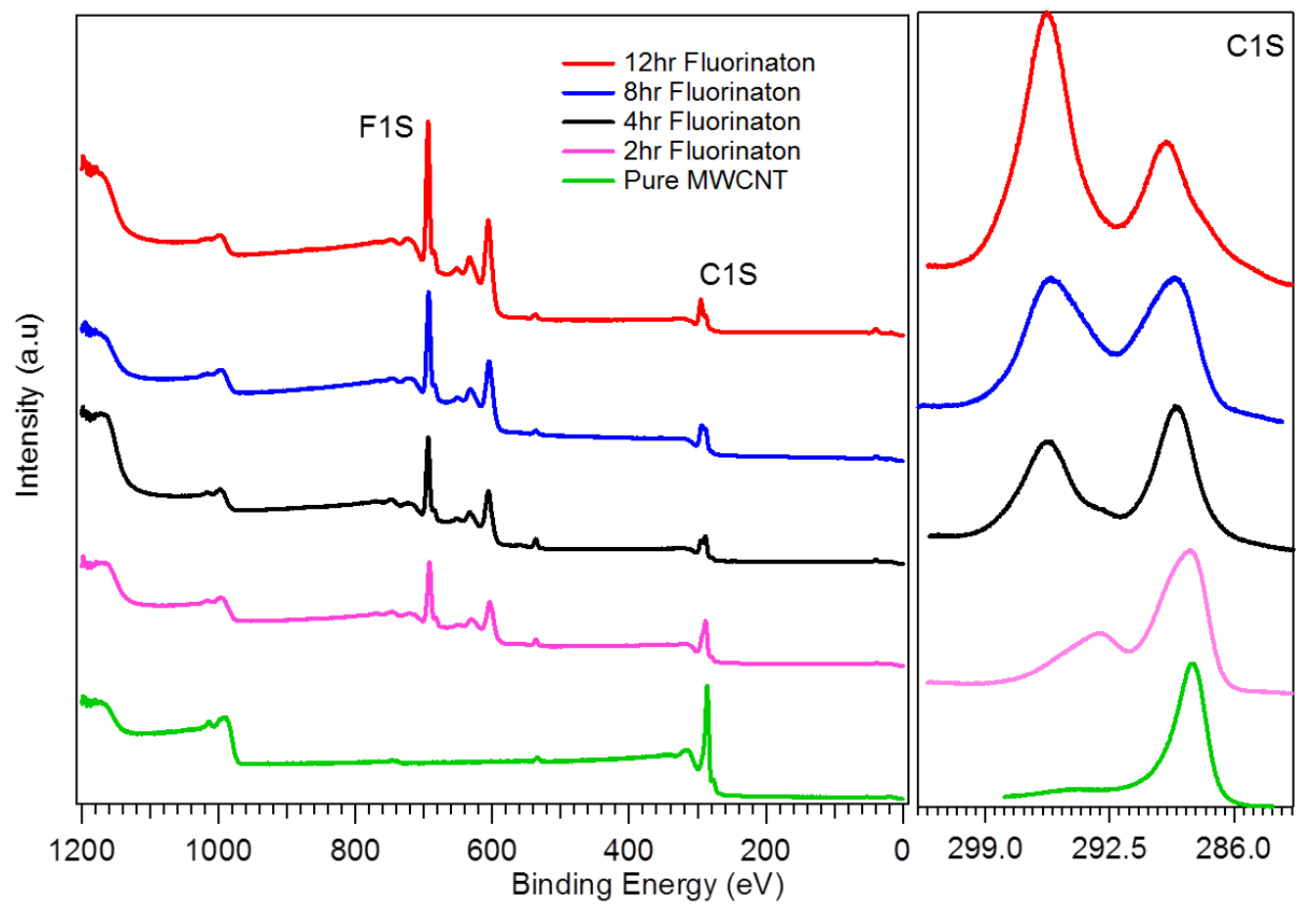

Figure 5.5: (a) Survey XPS spectra of pristine and controlled fluorinated MWNTs. (b) C 1s spectra of pristine and fluorinated MWNT samples

The XPS spectra of the MWCNTs used in this study were obtained with a MultiLab 3000 spectrometer (Thermo electron corporation, England). The spectra were analyzed in order to study the nature of the chemical species on the surface of 
MWCNTs after the fluorination using $\mathrm{Al} \mathrm{K \alpha}(1485.6 \mathrm{eV}) \mathrm{X}$-ray produced at $14.9 \mathrm{keV}$ of anode voltage. All measurements were obtained at $10^{-9}$ Torr chamber pressure.

XPS survey graphs of pure and fluorinated MWCNT samples are plotted in Figure 5.5a. All spectra show distinct carbon and oxygen peaks around $285.0 \mathrm{eV}$ and 533.0 $\mathrm{eV}$ respectively. Fluorine peaks were also found around $687.7 \mathrm{eV}$ on fluorinated MWCNTs. The atomic ratio of carbon and fluorine on the surface of MWCNTs extracted from the analysis of each peak are listed in Table 5.1.

\begin{tabular}{|c|c|c|c|}
\hline & Elem & Wt \% & At \% \\
\hline \multirow{2}{*}{2 hr Fluorination } & C-K & 93.77 & 95.97 \\
\cline { 2 - 4 } & F-K & $\mathbf{6 . 2 3}$ & 4.03 \\
\hline \multirow{2}{*}{$4 \mathrm{hr}$} & C-K & 70.66 & 79.2 \\
\cline { 2 - 4 } & F-K & $\mathbf{2 9 . 3 4}$ & 20.8 \\
\hline \multirow{2}{*}{$8 \mathrm{hr}$} & C-K & 52.38 & 63.5 \\
\cline { 2 - 4 } & F-K & $\mathbf{4 7 . 6 2}$ & 36.5 \\
\hline \multirow{2}{*}{$12 \mathrm{hr}$} & C-K & 31.38 & 85.2 \\
\hline & F-K & $\mathbf{6 8 . 6 2}$ & 14.8 \\
\hline
\end{tabular}

Table 5.1: atomic and weight ratio of carbon and fluorine on the surface of MWCNTs

The carbon concentration of samples dramatically decreased after fluorination while fluorine atomic ratio for $2 \mathrm{hr}, 4 \mathrm{hr}, 8 \mathrm{hr}$ and $12 \mathrm{hr}$ increased systematically implying that higher fluorine concentration can be achieved by increasing the exposure time.

Details of the $\mathrm{C} 1 \mathrm{~s}$ peak as a result of fluorination is shown in Fig. 5.5b. The peak with binding energy $\sim 284.6 \mathrm{eV}$ corresponds to carbon $\mathrm{sp}^{2}$ characteristic of graphene sheets. This feature starts to decrease as the fluorination progresses while a second peak with binding energy $\sim 289.4 \mathrm{eV}$ emerges and grows in intensity. This second 
peak corresponds to the carbon $\mathrm{sp}^{3}$ character due to the formation of carbon-fluorine bonding.

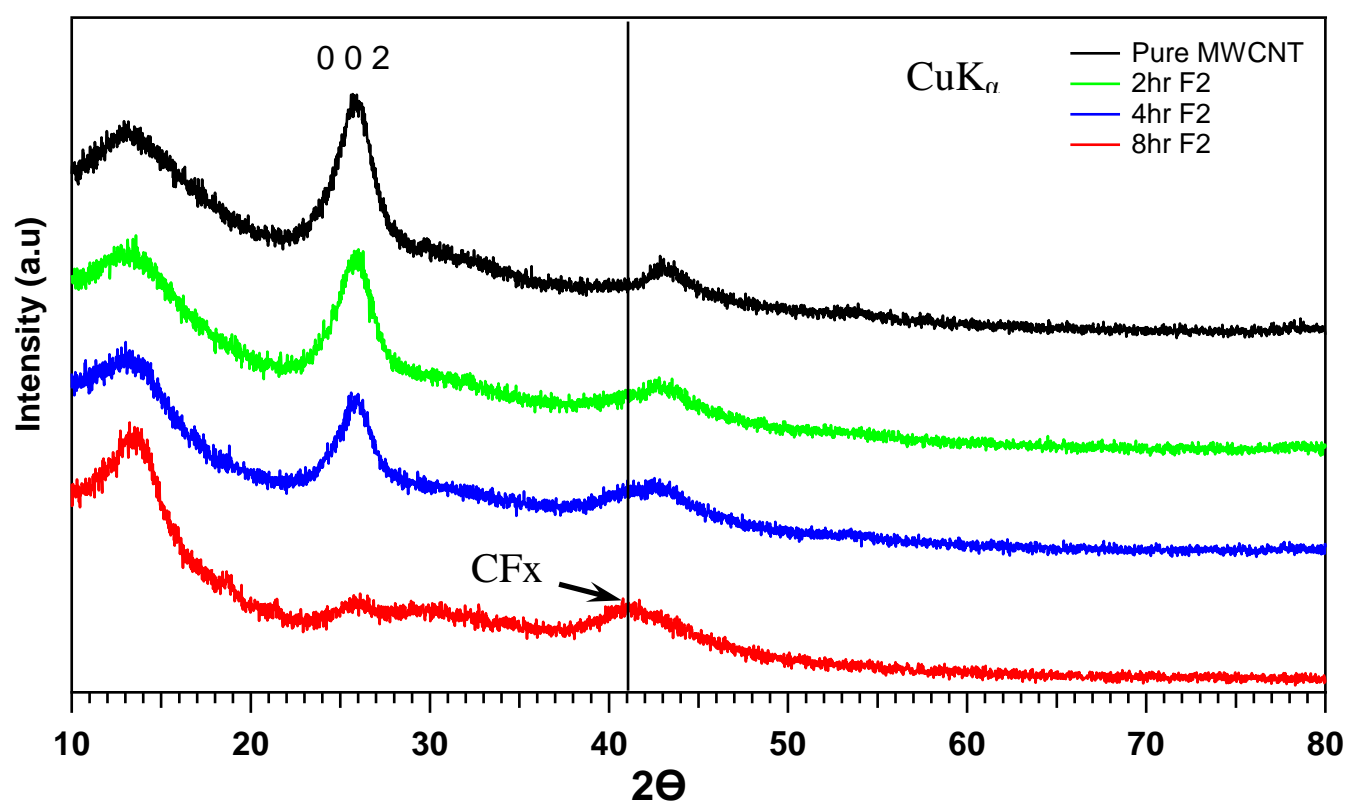

Figure 5.6: X-ray diffraction patterns of the F-MWNT samples.

X-ray diffraction patterns of fluorinated samples are shown in Figure 5.6. It is clear that fluorination reduces intensity of the most $\left(\begin{array}{lll}0 & 0 & 2\end{array}\right)$ peak of carbon nanotubes. Fluorination results in occurrence of new peak, which is labeled as CFx in Figure 5.6. The intensity of this peak increases with the degree of fluorination. This peak corresponds to the peak ( $\left.\begin{array}{lll}1 & 0 & 0\end{array}\right)$ for fluorinated nanotubes[195]. This peak is evident for the non-destructed structure of MWCNT. However, absence of peak corresponding to peak $\left(\begin{array}{lll}0 & 0 & 2\end{array}\right)$ in fully fluorinated sample implies that excessive fluorination destroys graphite-like structure of pristine MWCNT. Fluorination affects only the first few layers of the MWCNTs resulting in increased interlayer distances. With excessive fluorination, these layers separate from the parent tube and therefore no longer have graphite-like structure. 


\subsection{Primary battery (Li/CFx ) performance}

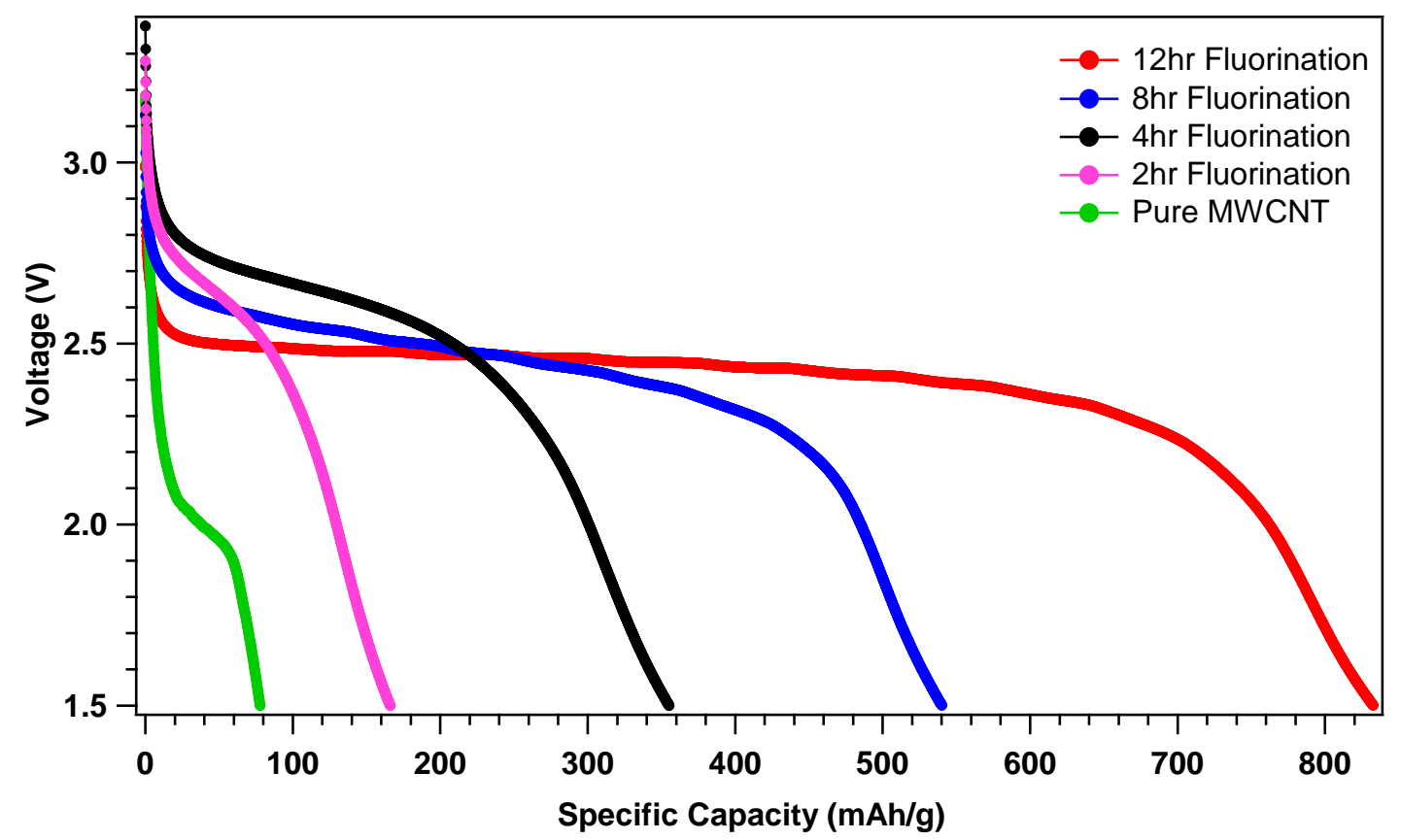

Figure 5.7: First discharge capacity curves of CFx Lithium primary battery.

CFx was used as the active cathode material in a primary battery. A CFx electrode film was prepared by coating a slurry composed of $85 \% \mathrm{CFx}, 10 \%$ carbon black and $5 \%$ poly (vinylidene fluoridecohexa fluoropropylene) in $\mathrm{N}$-methylpyrrolidone solvent onto a $\mathrm{Al}$ foil. The coating was dried in an $80{ }^{\circ} \mathrm{C}$ oven to evaporate solvent, and the resulting electrode film was either punched into small disks with a diameter of $2 \mathrm{~cm}^{2}$ for test in button cells for test in electrochemical cells. The Li metal was used as an anode electrode. The shaped electrode pieces were further dried at $100{ }^{\circ} \mathrm{C}$ under vacuum for $8 \mathrm{~h}$, and then transferred to a glove-box for cell assembly. In the glovebox $\mathrm{Li} / \mathrm{CFx}$ button cells were assembled for discharge tests by using a $0.5 \mathrm{~m} \mathrm{LiBF}_{4}$ solution in a 1:1 (weight) mixture of propylene carbonate (PC) and dimethoxyethane (DME) as the electrolyte and a Celgard ${ }^{\circledR} 3500$ membrane as the separator. Discharge and OCV tests were performed on a Arbin battery cycler. In all discharge tests, the cutoff voltage was set at $1.5 \mathrm{VThe}$ results for pristine, partially fluorinated, and fully 
fluorinated MWNTs are shown in Figure 5.7. The first cycle discharge capacity is seen to increase as the fluorination progresses. For the highest fluorinated sample, a specific capacity of $865 \mathrm{mAhg}^{-1}$ was achieved. This number remains one of the largest capacity values reported for $\mathrm{CFx}$ batteries to date.

\subsection{Secondary battery ( $\mathrm{Li} / \mathrm{CFx})$ performance}

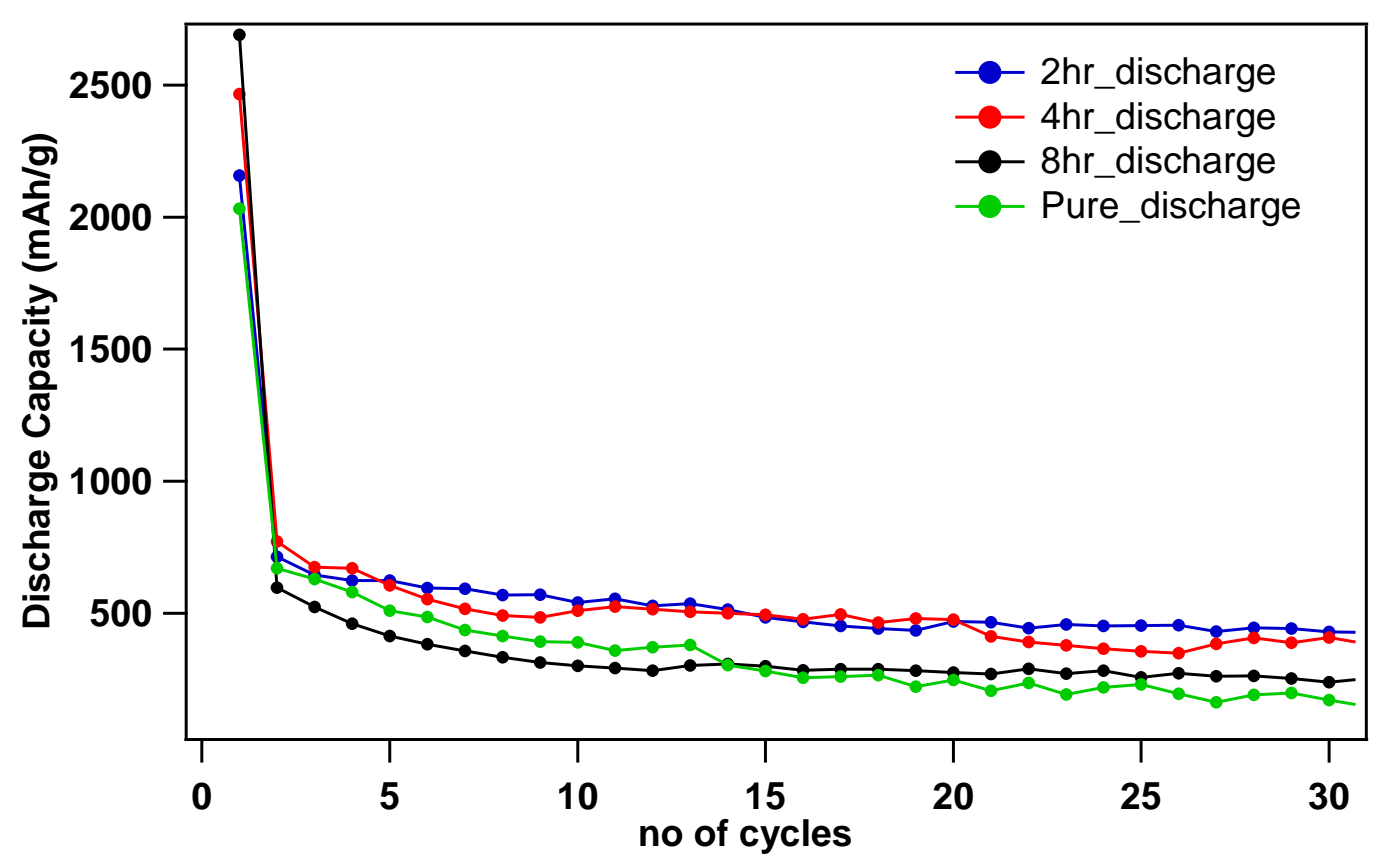

Figure 5.8: Cycling stability of the MWCNT with different fluorine concentration

Next we tested the samples for secondary Li-ion battery performances using CFx as the anode and $\mathrm{Li}$ as the cathode. Figure 5.8 shows the capacity when they were charged/discharged for 30 cycles between $3 \mathrm{~V}$ and $10 \mathrm{mV}$. Fully fluorinated sample shows low rechargeable capacity compared to the subfluorinated samples. Preliminary results show that mildly fluorinated ( $2 \mathrm{hr}$ ) MWNTs show high capacity $\sim 750 \mathrm{mAh} / \mathrm{g}$ and shows stable cycleability after 30 cycles. It shows $\sim 60.2 \%$ capacity retention over 30 cycles. But pristine MWCNT shows starting capacity of $\sim 650 \mathrm{mAh} / \mathrm{g}$ and with retention capacity being $\sim 150 \mathrm{mAh} / \mathrm{g}$ after 30 cycles. Pure MWCNT shows 
$25.5 \%$ capacity retention over 30 cycles. The $4 \mathrm{hr}$ and $8 \mathrm{hr}$ MWCNT shows capacity retention of $52.7 \%$ and $35.5 \%$ respectively. Several coin cells with different fluorine concentrations were tested. The $2 \mathrm{hr}$ fluorination exhibited similar high initial capacities, good cycle stabilities, and excellent capacity retentions at high rates.
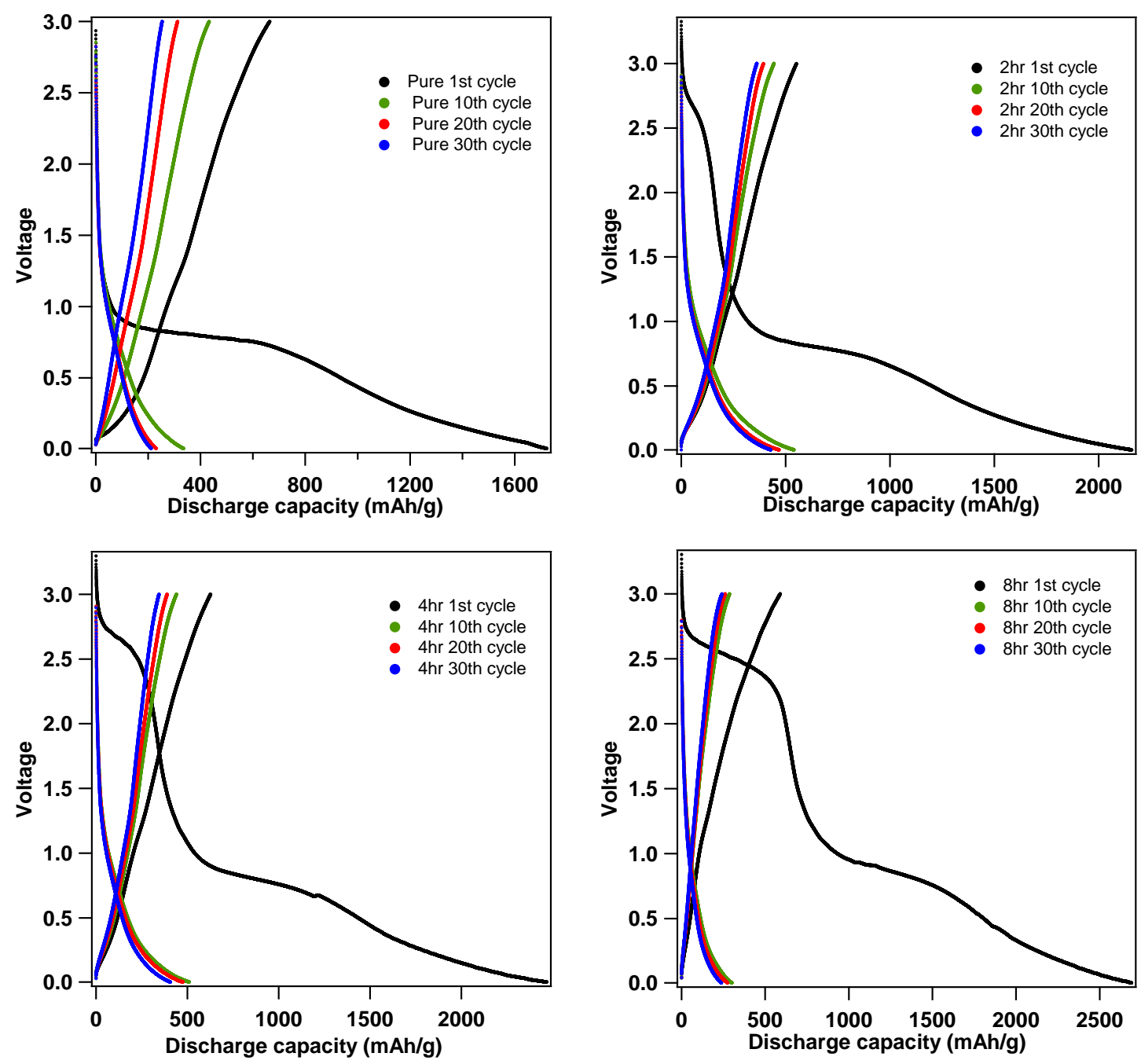

Figure 5.9: Discharge and charge profiles of the pure, $2 \mathrm{hr}$, 4hr, and $8 \mathrm{hr}$ MWCNTs obtained after one, ten, twenty and thirty cycles.

Figure 5.9 shows the Discharge and charge profiles for MWCNTs with different concentrations of fluorine obtained after one, ten, twenty and thirty cycles. First columbic efficiency is associated with the irreversible capacity loss in the 1 st cycle. This process involves electrolyte decomposition and subsequent formation of surface 
film called solid electrolyte interface (SEI) on anode. Some of the lithium ions are consumed during the formation of SEI, and therefore are not inserted in MWCNT surface. This is one of the challenges on developing carbonaceous anodes. Good anode material should suppress the electrochemical decomposition of solvents for the formation of SEI. Since electrochemical reactions take place at the surface of electrodes, surface structure is an important factor determining the electrochemical characteristics of carbon materials.

The $8 \mathrm{hr}$ fluorination shows a higher $1^{\text {st }}$ cycle discharge capacity. This particular behavior may be related to the fluorine induced disorder of the MWCNT structure. The cathode reduction of fluorinated MWCNT is limited by the lithium ions diffusion within the multi-tubes structure. Mild fluorinated MWCNT has less structure damages. Therefore, the lithium diffusion is hindered by a low defects density. For highly fluorinated MWCNT, additional intercalated fluorine creates more strains for their accommodation. This will lead to increased defect density. Defects such as cracks in the carbon layers and opening of the nanotube ends are additional paths for lithium ions to diffuse within the core of the fluorinated structure and access the electrochemically active $\mathrm{C}-\mathrm{F}$ sites. The presence of the non-fluorinated carbons in the fluorinated MWCNT core even in small amounts favors higher electron flow and accordingly enhances the electrode reaction kinetics. 

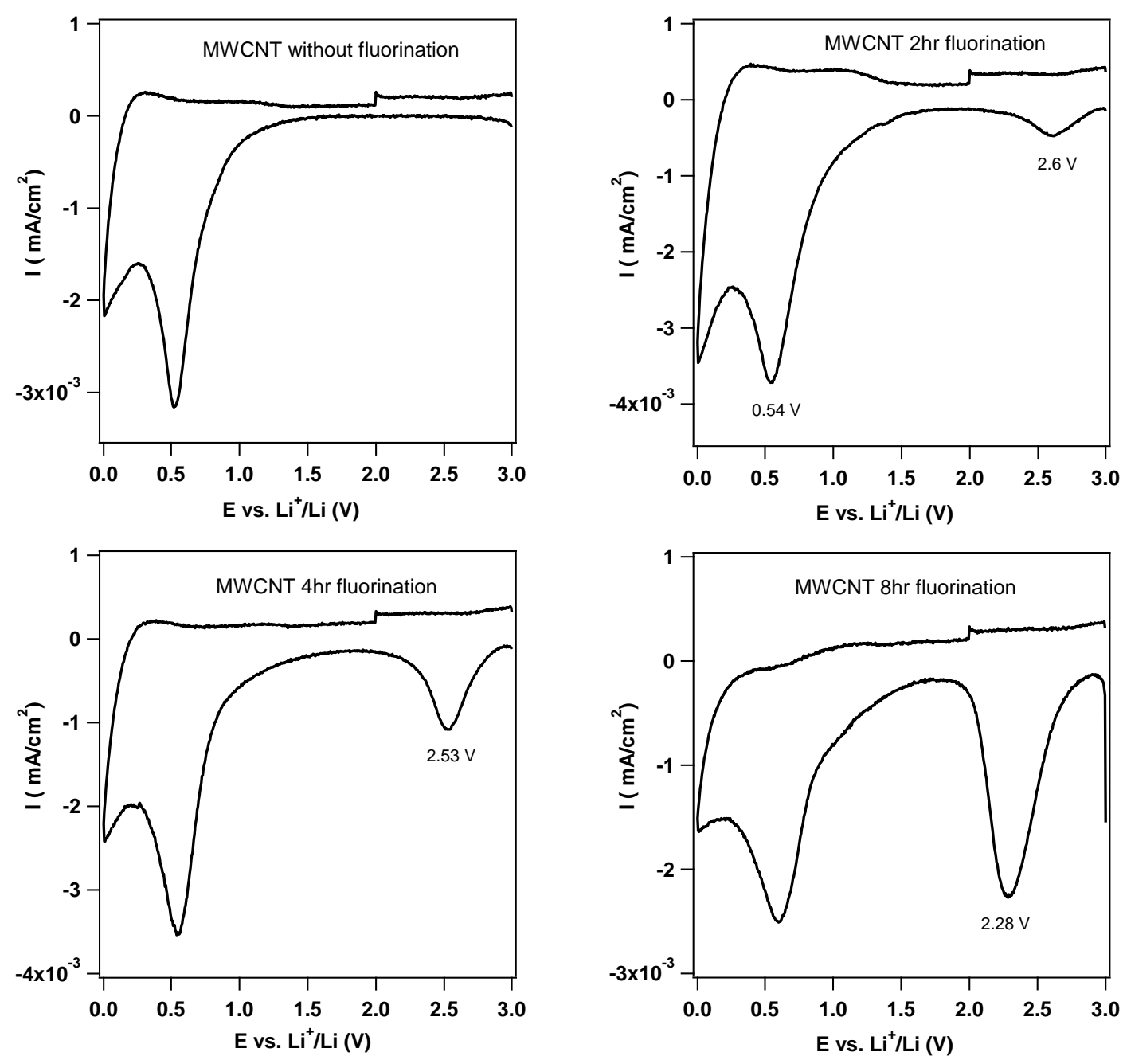

Figure 5.10: Cyclic voltammograms for the $1^{\text {st }}$ cycle of pure and plasma fluorinated MWCNT

Figure 5.10 shows the cyclic voltammograms obtained for pure and fluorinated MWCNT. A large cathodic current peak was found at $0.6 \mathrm{~V}$. This is an indication of the electrochemical reduction of electrolyte and subsequent formation of solid electrolyte interface (SEI). Higher fluorine concentration will reduce this peak implying that SEI is formed by the decomposition of less amounts of electrolyte. Another large cathodic peak shows up at $\sim 2.6 \mathrm{~V}$. This cathodic peak was enhanced and shifted with increasing plasma-treatment time as shown in Figure 5.9. 


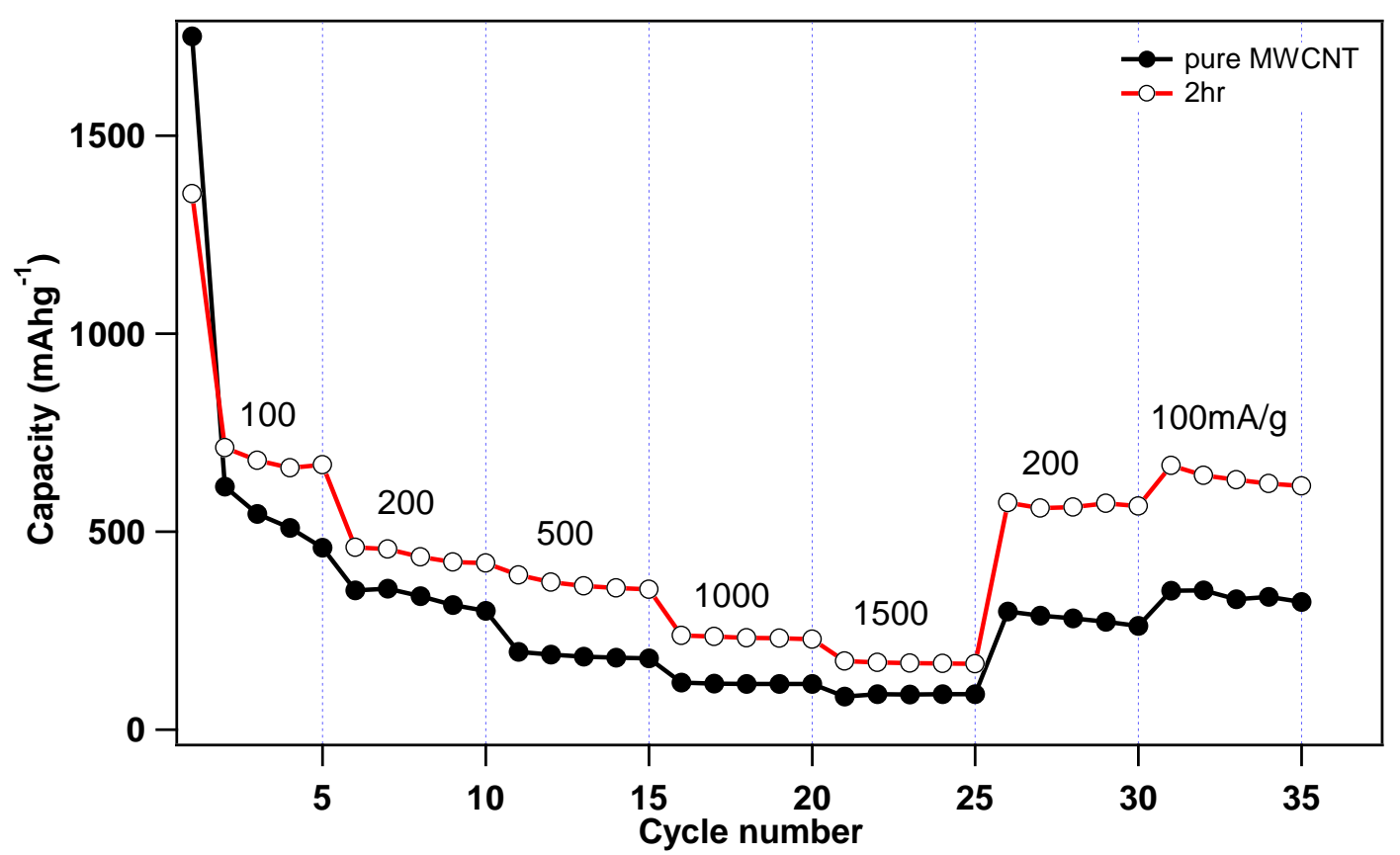

Figure 5.11: Cycling performance of the Pure and fluorinated MWCNT at various current densities.

The $2 \mathrm{hr}$ fluorinated MWCNT also has excellent rate performance. Figure 5.11 show that the capacity at $100 \mathrm{~mA} \mathrm{~g}^{-1}$ current density is $712 \mathrm{mAh} \mathrm{g}^{-1}$ and $172 \mathrm{mAh} \mathrm{g}^{-1}$ at $1.5 \mathrm{~A} \mathrm{~g}^{-1}$. The capacity retention is $\sim 80 \%$ after testing for 35 cycles. Five formation cycles at $100 \mathrm{~mA} \mathrm{~g}^{-1}, 5$ cycles each at current densities of $200 \mathrm{~mA} \mathrm{~g}^{-1}, 500 \mathrm{~mA} \mathrm{~g}^{-1}$, $1000 \mathrm{~mA} \mathrm{~g}^{-1}$ and $1.5 \mathrm{~A} \mathrm{~g}^{-1}$. This demonstrates the recovery of the mild fluorinated MWCNT after subjecting it to different charge-discharge rates. 


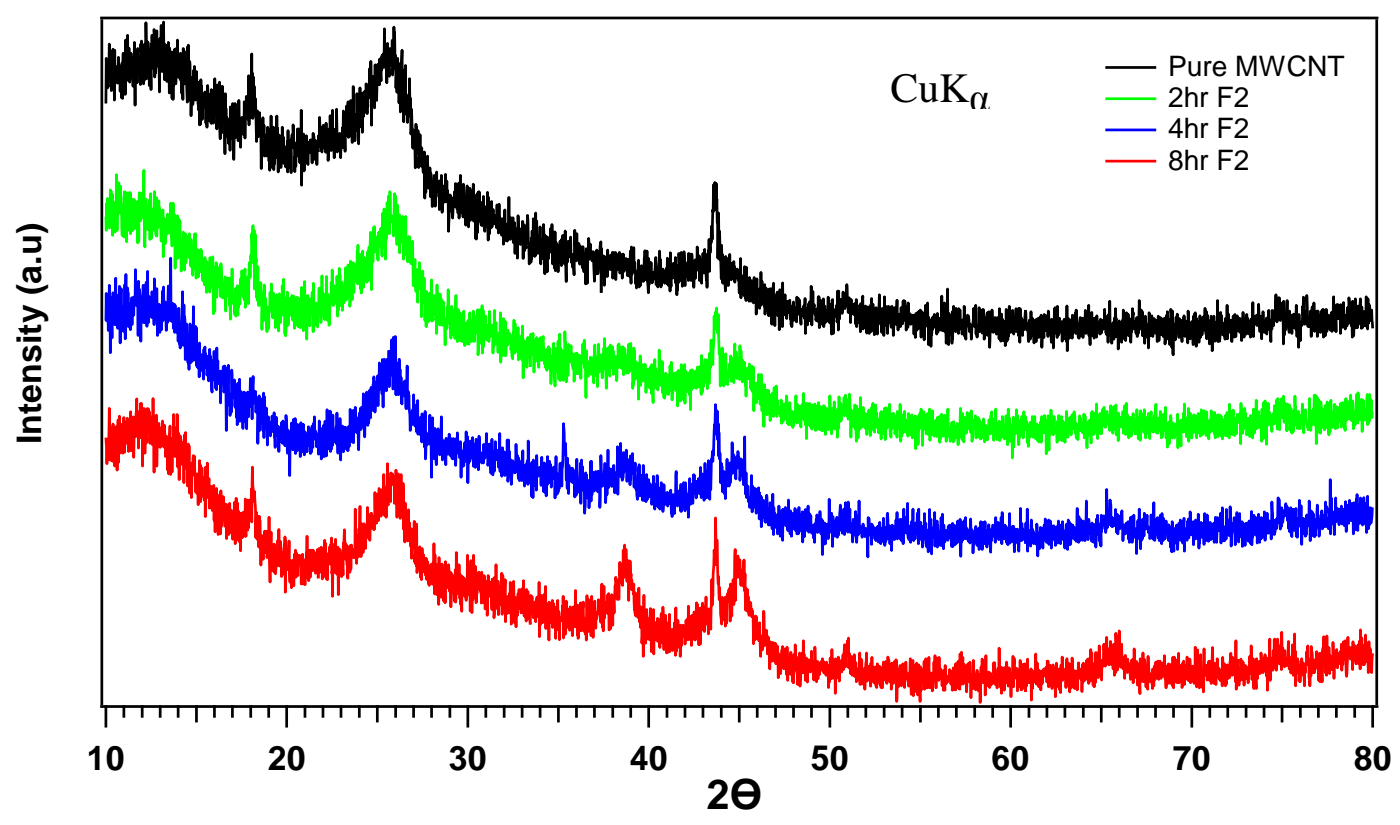

Figure 5.12: Ex-situ XRD for pristine and fluorinated MWNTs after first cycle discharge

Figure 5.12 shows the ex-situ XRD for pristine and fluorinated MWNTs after first cycle discharge. The (002) peak is seen to broaden as due the Li insertion between graphitic layers during the discharge. The emergence of new peaks $\sim 380$ and 45 is tentatively assigned for the formation of $\mathrm{Li}$ alloys with carbon and fluorine. 


\section{CHAPTER 06 CONCLUSIONS}

Monolayer Graphene synthesized by chemical vapor deposition was subjected to controlled and sequential hydrogenation using RF plasma while monitoring its electrical properties in-situ. Low temperature transport properties, viz., electrical resistance $(R)$, thermopower $(S)$, Hall mobility $(\mu)$, and magneto-resistance $(M R)$ were measured for each sample and correlated with ex-situ Raman scattering and X-ray photoemission (XPS) characteristics. For weak-hydrogenation, the transport is seen to be governed by electron diffusion and low temperature transport properties show metallic behavior (conductance, $G$ remains non-zero as $T \rightarrow 0$ ). For stronghydrogenation, the transport is found to be describable by variable range hopping (VRH) and the low $T$ conductivity shows insulating behavior $(G \rightarrow 0$ as $T \rightarrow 0$ ). Scaling functions for metallic and insulating graphene has been developed by identifying the critical hydrogen concentration. Weak localization (WL) behavior is seen with a negative $M R$ for weakly-hydrogenated Graphene and this $W L$ effects are seen to diminish as the hydrogenation progresses. A clear transition to strong localization $(S L)$ is evident with the emergence of pronounced negative $M R$ for strongly-hydrogenated Graphene.

Although graphene can be successfully hydrogenated, it can loose hydrogen at moderate temperatures. This will limit the use of this material in applications where high temperature stability is required. Unlike hydrogen, fluorine has higher binding energy to carbon and higher desorption energy than hydrogen. Sequential fluorination 
was done on monolayer graphene by $\mathrm{CF}_{4}$ plasma. Transport properties were measured to understand the effect of fluorination of graphene. Dominant transport mechanism for weak fluorination was found to be electron diffusion and low temperature transport for strong fluorination is governed by variable range hopping. A clear transition from weak localization to strong localization is evident with the emergence of pronounced negative magneto resistance for strongly fluorinated graphene. As determined by the high temperature resistance behavior, an appearance of a small band gap is observed and the band gap is seen to increase as the fluorination progresses.

Fluorine functionalization setup was further modified to prepare CFx battery electrode materials. Fully fluorinated MWCNT shows low rechargeable capacity compared to the subfluorinated samples. Mildly fluorinated (2hr) MWNTs show high capacity and shows better stability during charge discharge cycles. High concentrations of fluorine seems to suppress the capacity retention due to the to increased defect densities. While these defects of nanotubes will provide additional paths for lithium ions to diffuse within the core of the fluorinated structure and access the electrochemically active $\mathrm{C}-\mathrm{F}$ sites. So the first cycle discharge capacity is seen to increase as the fluorination progresses. Finally, controllably fluorinated carbon nanotubes showed promise for high capacity primary and secondary battery performances. 


\section{REFERENCES}

1. Fuchs, J.-N. and M.O. Goerbig, Introduction to the physical properties of graphene. Lecture Notes, 2008.

2. ELDREDGE, P. and B. AVERILL, Chemistry, principles, patterns, and applications: united states edition. 2005.

3. Wallace, P.R., The Band Theory of Graphite. Physical Review, 1947. 71(9): p. 622634.

4. Geim, A.K. and K.S. Novoselov, The rise of graphene. Nat Mater, 2007. 6(3): p. 183191.

5. Novoselov, K.S., et al., Electric Field Effect in Atomically Thin Carbon Films. Science, 2004. 306(5696): p. 666-669.

6. Landau, L.D., Zur Theorie der phasenumwandlungen II. Phys. Z. Sowjetunion, 1937. 11: p. 26-35.

7. Peierls, R.E., Quelques proprietes typiques des corpses solides. Ann. I. H. Poincare, 1935. 5: p. 177-222.

8. Partoens, B. and F.M. Peeters, From graphene to graphite: Electronic structure around the K point. Physical Review B, 2006. 74(7): p. 075404.

9. Castro Neto, A.H., et al., The electronic properties of graphene. Reviews of Modern Physics, 2009. 81(1): p. 109-162.

10. Eizenberg, M. and J. Blakely, Carbon monolayer phase condensation on Ni (111). Surface Science, 1979. 82(1): p. 228-236.

11. Novoselov, K.S., et al., A roadmap for graphene. Nature, 2012. 490(7419): p. 192200.

12. Lu, X.K., et al., Tailoring graphite with the goal of achieving single sheets. Nanotechnology, 1999. 10(3): p. 269-272.

13. Britnell, L., et al., Field-Effect Tunneling Transistor Based on Vertical Graphene Heterostructures. Science, 2012. 335(6071): p. 947-950.

14. Li, Z.Q., et al., Dirac charge dynamics in graphene by infrared spectroscopy. Nature Physics, 2008. 4(7): p. 532-535.

15. Ishibashi, T., et al., InP/InGaAs uni-traveling-carrier photodiodes. Ieice Transactions on Electronics, 2000. E83C(6): p. 938-949. 
16. Ishikawa, Y. and K. Wada, Near-Infrared Ge Photodiodes for Si Photonics: Operation Frequency and an Approach for the Future. Ieee Photonics Journal, 2010. 2(3): p. 306-320.

17. Xia, F., et al., Photocurrent Imaging and Efficient Photon Detection in a Graphene Transistor. Nano Letters, 2009. 9(3): p. 1039-1044.

18. Mueller, T., F. Xia, and P. Avouris, Graphene photodetectors for high-speed optical communications. Nature Photonics, 2010. 4(5): p. 297-301.

19. Echtermeyer, T.J., et al., Strong plasmonic enhancement of photovoltage in graphene. Nature Communications, 2011. 2.

20. Reed, G.T., et al., Silicon optical modulators. Nat Photon, 2010. 4(8): p. 518-526.

21. Liao, L., et al., $40 \mathrm{Gbit} / \mathrm{s}$ silicon optical modulator for highspeed applications. Electronics Letters, 2007. 43(22): p. 1196-1197.

22. Berger, C., et al., Ultrathin Epitaxial Graphite: 2D Electron Gas Properties and a Route toward Graphene-based Nanoelectronics. The Journal of Physical Chemistry B, 2004. 108(52): p. 19912-19916.

23. Emtsev, K.V., et al., Towards wafer-size graphene layers by atmospheric pressure graphitization of silicon carbide. Nat Mater, 2009. 8(3): p. 203-207.

24. Tromp, R.M. and J.B. Hannon, Thermodynamics and Kinetics of Graphene Growth on SiC(0001). Physical Review Letters, 2009. 102(10).

25. Hupalo, M., E.H. Conrad, and M.C. Tringides, Growth mechanism for epitaxial graphene on vicinal 6H-SiC(0001) surfaces: A scanning tunneling microscopy study. Physical Review B, 2009. 80(4): p. 041401.

26. Robinson, J., et al., Nucleation of Epitaxial Graphene on SiC(0001). ACS Nano, 2009. 4(1): p. 153-158.

27. Norimatsu, W. and M. Kusunoki, Formation process of graphene on SiC (0001). Physica E-Low-Dimensional Systems \& Nanostructures, 2010. 42(4): p. 691-694.

28. VanMil, B.L., et al., Etch rates near hot-wall CVD growth temperature for Si-face 4H-SiC using H2 and C3H8. Journal of Crystal Growth, 2009. 311(2): p. 238-243.

29. Dikin, D.A., et al., Preparation and characterization of graphene oxide paper. Nature, 2007. 448(7152): p. 457-460.

30. Park, S., et al., Graphene oxide papers modified by divalent ions - Enhancing mechanical properties via chemical cross-linking. Acs Nano, 2008. 2(3): p. 572-578.

31. Li, D., et al., Processable aqueous dispersions of graphene nanosheets. Nat Nano, 2008. 3(2): p. 101-105.

32. Park, S., et al., Aqueous Suspension and Characterization of Chemically Modified Graphene Sheets. Chemistry of Materials, 2008. 20(21): p. 6592-6594.

33. Chen, H., et al., Mechanically strong, electrically conductive, and biocompatible graphene paper. Advanced Materials, 2008. 20(18): p. 3557-+. 
34. Stankovich, S., et al., Graphene-based composite materials. Nature, 2006. 442(7100): p. 282-286.

35. Stoller, M.D., et al., Graphene-Based Ultracapacitors. Nano Letters, 2008. 8(10): p. 3498-3502.

36. Wang, X., L. Zhi, and K. Muellen, Transparent, conductive graphene electrodes for dye-sensitized solar cells. Nano Letters, 2008. 8(1): p. 323-327.

37. Buchsteiner, A., A. Lerf, and J. Pieper, Water dynamics in graphite oxide investigated with neutron scattering. Journal of Physical Chemistry B, 2006. 110(45): p. 2232822338.

38. Stankovich, S., et al., Stable aqueous dispersions of graphitic nanoplatelets via the reduction of exfoliated graphite oxide in the presence of poly(sodium 4styrenesulfonate). Journal of Materials Chemistry, 2006. 16(2): p. 155-158.

39. Kuzmenko, A.B., et al., Universal optical conductance of graphite. Physical Review Letters, 2008. 100(11).

40. Jariwala, D., A. Srivastava, and P.M. Ajayan, Graphene synthesis and band gap opening. Journal of Nanoscience and Nanotechnology, 2011. 11(8): p. 6621-6641.

41. Zhou, S.Y., et al., Substrate-induced bandgap opening in epitaxial graphene. Nat Mater, 2007. 6(10): p. 770-775.

42. Kawasaki, T., et al., Double atomic layers of graphene/monolayer h-BN on Ni(111) studied by scanning tunneling microscopy and scanning tunneling spectroscopy. Surface Review and Letters, 2002. 9(3-4): p. 1459-1464.

43. Giovannetti, G., et al., Publisher's Note: Substrate-induced band gap in graphene on hexagonal boron nitride: Ab initio density functional calculations (vol 76, artn 073103, 2007). Physical Review B, 2007. 76(7).

44. Shemella, P. and S.K. Nayak, Electronic structure and band-gap modulation of graphene via substrate surface chemistry. Applied Physics Letters, 2009. 94(3).

45. Wei, D., et al., Synthesis of N-Doped Graphene by Chemical Vapor Deposition and Its Electrical Properties. Nano Letters, 2009. 9(5): p. 1752-1758.

46. Panchakarla, L.S., A. Govindaraj, and C.N.R. Rao, Boron- and nitrogen-doped carbon nanotubes and graphene. Inorganica Chimica Acta, 2010. 363(15): p. 41634174.

47. Yu, S.S., et al., First principle calculations of the electronic properties of nitrogendoped carbon nanoribbons with zigzag edges. Carbon, 2008. 46(3): p. 537-543.

48. $\quad \mathrm{Ci}, \mathrm{L}$. , et al., Atomic layers of hybridized boron nitride and graphene domains. Nature Materials, 2010. 9(5): p. 430-435.

49. Li, J. and V.B. Shenoy, Graphene quantum dots embedded in hexagonal boron nitride sheets. Applied Physics Letters, 2011. 98(1). 
50. Ma, X., et al., Semi-empirical studies on electronic structures of a boron-doped graphene layer - Implications on the oxidation mechanism. Carbon, 1997. 35(10-11): p. $1517-1525$.

51. Endo, M., et al., Scanning tunneling microscope study of boron-doped highly oriented pyrolytic graphite. Journal of Applied Physics, 2001. 90(11): p. 5670-5674.

52. Weng-Sieh, Z., et al., Synthesis of $B_{-}\{x\} C_{-}\{y\} N_{-}\{z\}$ nanotubules. Physical Review B, 1995. 51(16): p. 11229-11232.

53. Liu, A.Y., R.M. Wentzcovitch, and M.L. Cohen, Atomic arrangement and electronic structure of BC_\{2\}N. Physical Review B, 1989. 39(3): p. 1760-1765.

54. Miyamoto, Y., et al., Chiral tubules of hexagonal BC_\{2\}N. Physical Review B, 1994. 50(7): p. 4976-4979.

55. Haddon, R., et al., Purification and separation of carbon nanotubes. Mrs Bulletin, 2004. 29(04): p. 252-259.

56. Kaner, R., et al., Boron-carbon-nitrogen materials of graphite-like structure. Materials research bulletin, 1987. 22(3): p. 399-404.

57. Kawaguchi, M., T. Kawashima, and T. Nakajima, Syntheses and Structures of New Graphite-like Materials of Composition $B C N(H)$ and $B C 3 N(H)$. Chemistry of Materials, 1996. 8(6): p. 1197-1201.

58. $\quad \mathrm{Yu}$, J., et al., Turbostratic boron carbonitride films produced by bias-assisted hot filament chemical vapor deposition. Journal of Applied Physics, 2000. 87(8): p. 40224025.

59. Enouz, S., et al., C-BN Patterned Single-Walled Nanotubes Synthesized by Laser Vaporization. Nano Letters, 2007. 7(7): p. 1856-1862.

60. Ivanovskaya, V.V., et al., BN Domains Included into Carbon Nanotubes: Role of Interface. The Journal of Physical Chemistry C, 2009. 113(38): p. 16603-16609.

61. Ding, Y., Y. Wang, and J. Ni, Electronic properties of graphene nanoribbons embedded in boron nitride sheets. Applied Physics Letters, 2009. 95(12).

62. $\mathrm{Ci}, \mathrm{L}$. , et al., Atomic layers of hybridized boron nitride and graphene domains. Nat Mater, 2010. 9(5): p. 430-435.

63. Jiao, L., et al., Narrow graphene nanoribbons from carbon nanotubes. Nature, 2009. 458(7240): p. 877-880.

64. Nakada, K., et al., Edge state in graphene ribbons: Nanometer size effect and edge shape dependence. Physical Review B, 1996. 54(24): p. 17954-17961.

65. Wakabayashi, K., et al., Electronic and magnetic properties of nanographite ribbons. Physical Review B, 1999. 59(12): p. 8271-8282.

66. Fujita, M., et al., Peculiar localized state at zigzag graphite edge. Journal of the Physical Society of Japan, 1996. 65(7): p. 1920-1923.

67. Berger, C., et al., Electronic confinement and coherence in patterned epitaxial graphene. Science, 2006. 312(5777): p. 1191-1196. 
68. Ponomarenko, L.A., et al., Chaotic dirac billiard in graphene quantum dots. Science, 2008. 320(5874): p. 356-358.

69. Kosynkin, D.V., et al., Longitudinal unzipping of carbon nanotubes to form graphene nanoribbons. Nature, 2009. 458(7240): p. 872-876.

70. Abrahams, E., et al., Scaling Theory of Localization: Absence of Quantum Diffusion in Two Dimensions. Physical Review Letters, 1979. 42(10): p. 673-676.

71. Licciardello, D. and D. Thouless, Constancy of minimum metallic conductivity in two dimensions. Physical Review Letters, 1975. 35(21): p. 1475-1478.

72. Balian, R.M., R. Maynard, and G. Toulouse, Ill-Condensed Matter. 1983: NorthHolland Publishing Company.

73. Ashcroft, N. and N. Mermin, Solid State Physics (Brooks Cole, 1976). Cited on: p. 26.

74. Lee, P.A. and T. Ramakrishnan, Disordered electronic systems. Reviews of Modern Physics, 1985. 57(2): p. 287.

75. Medvedyeva, M.V., On localization of Dirac fermions by disorder. 2011: Instituut Lorentz for Theoretical Physics, Leiden Institute of Physics (LION), Faculty of Science, Leiden University.

76. Abrahams, E., et al., Scaling theory of localization: Absence of quantum diffusion in two dimensions. Physical Review Letters, 1979. 42(10): p. 673-676.

77. Hikami, S., A.I. Larkin, and Y. Nagaoka, Spin-orbit interaction and magnetoresistance in the two dimensional random system. Progress of Theoretical Physics, 1980. 63(2): p. 707-710.

78. Pierson, H.O., Handbook of chemical vapor deposition: principles, technology and applications. 1999: William Andrew.

79. Miao, C., et al., Chemical Vapor Deposition of Graphene. Physics and Applications of Graphene-Experiments. Edited by Mikhailov S, Rijeka: InTech, 2011: p. 37-54.

80. Wei, D., et al., Synthesis of N-Doped Graphene by Chemical Vapor Deposition and Its Electrical Properties. Nano Letters, 2009. 9(5): p. 17521758.

81. Li, X., et al., Large-Area Synthesis of High-Quality and Uniform Graphene Films on Copper Foils. Science, 2009. 324(5932): p. 1312-1314.

82. Reina, A., et al., Large Area, Few-Layer Graphene Films on Arbitrary Substrates by Chemical Vapor Deposition. Nano Letters, 2009. 9(1): p. 30-35.

83. Kim, K.S., et al., Large-scale pattern growth of graphene films for stretchable transparent electrodes. Nature, 2009. 457(7230): p. 706-710. 
84. López, G.A. and E.J. Mittemeijer, The solubility of $C$ in solid $C u$. Scripta Materialia, 2004. 51(1): p. 1-5.

85. $\mathrm{Yu}, \mathrm{Q}$., et al., Graphene segregated on Ni surfaces and transferred to insulators. Applied Physics Letters, 2008. 93(11): p. 113103.

86. POSTEK, M.T., SCANNING ELECTRON MICROSCOPY A STUDENTS HANDBOOK. 1980.

87. Watt, I.M., The Principles and Practice of Electron Microscopy. 1997: Cambridge University Press.

88. Lyman, C.E., Scanning Electron Microscopy, X-ray Microanalysis, and Analytical Electron Microscopy: A Laboratory Workbook. 1990: Plenum Press.

89. Goldstein, J., Scanning electron microscopy and x-ray microanalysis. 2003: Springer London, Limited.

90. Everhart, T. and R. Thornley, Wide-band detector for micro-microampere low-energy electron currents. Journal of scientific instruments, 1960. 37(7): p. 246.

91. Bandhyopadhya, A. and S. Bose, Characterization of Biomaterials. 2013: Elsevier Science.

92. Ferrari, A.C., et al., Raman Spectrum of Graphene and Graphene Layers. Physical Review Letters, 2006. 97(18): p. 187401.

93. Gupta, A., et al., Raman scattering from high-frequency phonons in supported n-graphene layer films. Nano Letters, 2006. 6(12): p. 2667-2673.

94. Charlier, J.-C., et al., Electron and phonon properties of graphene: their relationship with carbon nanotubes, in Carbon nanotubes. 2008, Springer. p. 673-709.

95. Malard, L., et al., Probing the electronic structure of bilayer graphene by Raman scattering. Physical Review B, 2007. 76(20): p. 201401.

96. Ferralis, N., R. Maboudian, and C. Carraro, Evidence of structural strain in epitaxial graphene layers on 6H-SiC (0001). Physical review letters, 2008. 101(15): p. 156801.

97. Mohiuddin, T., et al., Uniaxial strain in graphene by Raman spectroscopy: $G$ peak splitting, Grüneisen parameters, and sample orientation. Physical Review B, 2009. 79(20): p. 205433. 
98. Huang, M., et al., Phonon softening and crystallographic orientation of strained graphene studied by Raman spectroscopy. Proceedings of the National Academy of Sciences, 2009. 106(18): p. 7304-7308.

99. Das, A., et al., Phonon renormalization in doped bilayer graphene. Physical Review B, 2009. 79(15): p. 155417.

100. Malard, L., et al., Observation of distinct electron-phonon couplings in gated bilayer graphene. Physical review letters, 2008. 101(25): p. 257401.

101. Das, A., et al., Monitoring dopants by Raman scattering in an electrochemically top-gated graphene transistor. Nature nanotechnology, 2008. 3(4): p. 210-215.

102. Compagnini, G., et al., Ion irradiation and defect formation in single layer graphene. Carbon, 2009. 47(14): p. 3201-3207.

103. Jorio, A. and L.G. Cançado, Perspectives on Raman spectroscopy of graphene-based systems: from the perfect two-dimensional surface to charcoal. Physical Chemistry Chemical Physics, 2012. 14(44): p. 1524615256.

104. Lucchese, M.M., et al., Quantifying ion-induced defects and Raman relaxation length in graphene. Carbon, 2010. 48(5): p. 1592-1597.

105. Dresselhaus, M., G. Dresselhaus, and M. Hofmann, Raman spectroscopy as a probe of graphene and carbon nanotubes. Philosophical Transactions of the Royal Society A: Mathematical, Physical and Engineering Sciences, 2008. 366(1863): p. 231-236.

106. Berciaud, S., et al., Probing the intrinsic properties of exfoliated graphene: Raman spectroscopy of free-standing monolayers. Nano letters, 2008. 9(1): p. 346-352.

107. Wang, Y., D.C. Alsmeyer, and R.L. McCreery, Raman spectroscopy of carbon materials: structural basis of observed spectra. Chemistry of Materials, 1990. 2(5): p. 557-563.

108. Thomsen, C. and S. Reich, Double resonant Raman scattering in graphite. Physical Review Letters, 2000. 85(24): p. 5214-5217.

109. Dresselhaus, M.S., et al., Perspectives on carbon nanotubes and graphene Raman spectroscopy. Nano letters, 2010. 10(3): p. 751-758.

110. Malard, L., et al., Raman spectroscopy in graphene. Physics Reports, 2009. 473(5): p. 51-87. 
111. Mattevi, C., H. Kim, and M. Chhowalla, A review of chemical vapour deposition of graphene on copper. Journal of Materials Chemistry, 2011. 21(10): p. 3324-3334.

112. Mikhailov, S., Physics and Applications of Graphene-Experiments. Tech Janeza Trdine, Rijeka, Croatia, 2011.

113. Leenaerts, O., B. Partoens, and F. Peeters, Water on graphene: Hydrophobicity and dipole moment using density functional theory. Physical Review B, 2009. 79(23): p. 235440.

114. Obraztsov, A., et al., Chemical vapor deposition of thin graphite films of nanometer thickness. Carbon, 2007. 45(10): p. 2017-2021.

115. Tyurnina, A.V., et al., Topology peculiarities of graphite films of nanometer thickness. physica status solidi (b), 2010. 247(11-12): p. 3010-3013.

116. Geim, A.K. and K.S. Novoselov, The rise of graphene. Nature Materials, 2007. 6(3): p. 183-191.

117. Yan, L., et al., Chemistry and physics of a single atomic layer: strategies and challenges for functionalization of graphene and graphene-based materials. Chemical Society Reviews, 2012. 41(1): p. 97-114.

118. Jin, M., et al., Synthesis and systematic characterization of functionalized graphene sheets generated by thermal exfoliation at low temperature. Journal of Physics DApplied Physics, 2010. 43(27).

119. Batzill, M., The surface science of graphene: Metal interfaces, CVD synthesis, nanoribbons, chemical modifications, and defects. Surface Science Reports, 2012. 67(3-4): p. 83-115.

120. Rao, C.N.R., et al., GRAPHENE: SYNTHESIS, FUNCTIONALIZATION AND PROPERTIES. Modern Physics Letters B, 2011. 25(7): p. 427-451.

121. Lue, P., et al., Recent progresses in application of functionalized graphene sheets. Science China-Technological Sciences, 2010. 53(9): p. 2311-2319.

122. Xu, Y., et al., Flexible graphene films via the filtration of water-soluble noncovalent functionalized graphene sheets. Journal of the American Chemical Society, 2008. 130(18): p. 5856-+.

123. Savchenko, A., MATERIALS SCIENCE Transforming Graphene. Science, 2009. 323(5914): p. 589-590.

124. Boukhvalov, D.W. and M.I. Katsnelson, Chemical Functionalization of Graphene with Defects. Nano Letters, 2008. 8(12): p. 4373-4379.

125. Niyogi, S., et al., Covalent Chemistry for Graphene Electronics. Journal of Physical Chemistry Letters, 2011. 2(19): p. 2487-2498.

126. Zhang, H., et al., Aryl Functionalization as a Route to Band Gap Engineering in Single Layer Graphene Devices. Nano Letters, 2011. 11(10): p. 4047-4051. 
127. Gomez-Navarro, C., et al., Electronic Transport Properties of Individual Chemically Reduced Graphene Oxide Sheets. (vol 7, pg 3499, 2007). Nano Letters, 2009. 9(5).

128. Elias, D.C., et al., Control of Graphene's Properties by Reversible Hydrogenation: Evidence for Graphane. Science, 2009. 323(5914): p. 610-613.

129. Ryu, S., et al., Reversible Basal Plane Hydrogenation of Graphene. Nano Letters, 2008. 8(12): p. 4597-4602.

130. Balog, R., et al., Bandgap opening in graphene induced by patterned hydrogen adsorption. Nature Materials, 2010. 9(4): p. 315-319.

131. Matis, B.R., et al., Giant negative magnetoresistance and a transition from strong to weak localization in hydrogenated graphene. Physical Review B, 2012. 85(19).

132. Burgess, J.S., et al., Tuning the electronic properties of graphene by hydrogenation in a plasma enhanced chemical vapor deposition reactor. Carbon, 2011. 49(13): p. 4420-4426.

133. Luo, Z.Q., et al., Thickness-Dependent Reversible Hydrogenation of Graphene Layers. Acs Nano, 2009. 3(7): p. 1781-1788.

134. Sachdev, S., Quantum phase transitions. Physics World, 1999. 12(4): p. 33-38.

135. Belitz, D. and T.R. Kirkpatrick, The Anderson-Mott transition. Reviews of Modern Physics, 1994. 66(2): p. 261-380.

136. van der PAUYV, L., A method of measuring specific resistivity and Hall effect of discs of arbitrary shape. 1958.

137. Sumanasekera, G.U., L. Grigorian, and P.C. Eklund, Low-temperature thermoelectrical power measurements using analogue subtraction. Measurement Science \& Technology, 2000. 11(3): p. 273-277.

138. Bae, S., et al., Roll-to-roll production of 30-inch graphene films for transparent electrodes. Nature Nanotechnology, 2010. 5(8): p. 574-578.

139. Li, X., et al., Transfer of Large-Area Graphene Films for High-Performance Transparent Conductive Electrodes. Nano Letters, 2009. 9(12): p. 4359-4363.

140. Sidorov, A.N., et al., Thermoelectric power of graphene as surface charge doping indicator. Applied Physics Letters, 2011. 99(1).

141. Chakrapani, V., et al., Charge transfer equilibria between diamond and an aqueous oxygen electrochemical redox couple. Science, 2007. 318(5855): p. 1424-1430.

142. Abrahams, E., et al., Scaling Theory of Localization: Absence of Quantum Diffusion in Two Dimensions. Physical Review Letters, 1979. 42(10): p. 673-676.

143. Lee, H.L., et al., Quantum-critical conductivity scaling for a metal-insulator transition. Science, 2000. 287(5453): p. 633-636.

144. v. Loehneysen, H., Electron-electron interactions and the metal-insulator transition in heavily doped silicon. Annalen Der Physik, 2011. 523(8-9): p. 599-611. 
145. Helgren, E., et al., Measurements of the complex conductivity of NbxSi(1-x) alloys on the insulating side of the metal-insulator transition. Physical review letters, 2001. 87(11): p. 116602-116602.

146. Mott, N.F., Conduction in non-crystalline materials. Philosophical Magazine, 1969. 19(160): p. 835-852.

147. Chen, Y.-F., et al., Magnetoresistance in single-layer graphene: weak localization and universal conductance fluctuation studies. Journal of Physics-Condensed Matter, 2010. 22(20).

148. Tikhonenko, F.V., et al., Weak localization in graphene flakes. Physical Review Letters, 2008. 100(5).

149. Ki, D.-K., et al., Inelastic scattering in a monolayer graphene sheet: A weaklocalization study. Physical Review B, 2008. 78(12).

150. McCann, E., et al., Weak-localization magnetoresistance and valley symmetry in graphene. Physical Review Letters, 2006. 97(14).

151. Datta, S., Electronic Transport in Mesoscopic Systems. 1997: Cambridge University Press.

152. Sakkopoulos, S., et al., THERMOPOWER SIGN REVERSAL VERSUS TEMPERATURE AND DC CONDUCTIVITY IN POLYANILINE DERIVATIVES. Journal of Physics-Condensed Matter, 1992. 4(9): p. 2231-2237.

153. Malard, L.M., et al., Raman spectroscopy in graphene. Physics Reports-Review Section of Physics Letters, 2009. 473(5-6): p. 51-87.

154. Cancado, L.G., et al., Quantifying Defects in Graphene via Raman Spectroscopy at Different Excitation Energies. Nano Letters, 2011. 11(8): p. 3190-3196.

155. Ferrari, A.C., Raman spectroscopy of graphene and graphite: Disorder, electronphonon coupling, doping and nonadiabatic effects. Solid State Communications, 2007. 143(1-2): p. 47-57.

156. Nikitin, A., et al., $\mathrm{C}-\mathrm{H}$ bond formation at the graphite surface studied with core level spectroscopy. Surface Science, 2008. 602(14): p. 2575-2580.

157. Zhang, Y.-Y., et al., Localization and the Kosterlitz-Thouless Transition in Disordered Graphene. Physical Review Letters, 2009. 102(10).

158. Nair, R.R., et al., Fluorographene: A Two-Dimensional Counterpart of Teflon. Small, 2010. 6(24): p. 2877-2884.

159. Hong, X., et al., Colossal negative magnetoresistance in dilute fluorinated graphene. Physical Review B, 2011. 83(8).

160. Robinson, J.T., et al., Properties of fluorinated graphene films. Nano letters, 2010. 10(8): p. 3001-3005.

161. Withers, F., M. Dubois, and A.K. Savchenko, Electron properties of fluorinated single-layer graphene transistors. Physical Review B, 2010. 82(7): p. 073403. 
162. Sumanasekera, G.U., L. Grigorian, and P.C. Eklund, Low-temperature thermoelectrical power measurements using analogue subtraction. Measurement Science \& Technology, 2000. 11(3): p. 273-277.

163. Bae, S., et al., Roll-to-roll production of 30-inch graphene films for transparent electrodes. Nature Nanotechnology, 2010. 5(8): p. 574-578.

164. Li, X., et al., Transfer of Large-Area Graphene Films for High-Performance Transparent Conductive Electrodes. Nano Letters, 2009. 9(12): p. 4359-4363.

165. Sidorov, A.N., et al., Thermoelectric power of graphene as surface charge doping indicator. Applied Physics Letters, 2011. 99(1).

166. Sidorov, A.N., et al., Charge transfer equilibria in ambient-exposed epitaxial graphene on (000(1)over-bar) $6 \mathrm{H}$-SiC (vol 111, 113706, 2012). Journal of Applied Physics, 2012. 112(2).

167. Lee, H.L., et al., Quantum-critical conductivity scaling for a metal-insulator transition. Science, 2000. 287(5453): p. 633-636.

168. v. Loehneysen, H., Electron-electron interactions and the metal-insulator transition in heavily doped silicon. Annalen Der Physik, 2011. 523(8-9): p. 599-611.

169. Helgren, E., et al., Measurements of the complex conductivity of NbxSi(1-x) alloys on the insulating side of the metal-insulator transition. Physical review letters, 2001. 87(11): p. 116602-116602.

170. Mott, N.F., Conduction in non-crystalline materials. Philosophical Magazine, 1969. 19(160): p. 835-852.

171. Matis, B.R., et al., Giant negative magnetoresistance and a transition from strong to weak localization in hydrogenated graphene. Physical Review B, 2012. 85(19).

172. Chen, Y.-F., et al., Magnetoresistance in single-layer graphene: weak localization and universal conductance fluctuation studies. Journal of Physics-Condensed Matter, 2010. 22(20).

173. Tikhonenko, F.V., et al., Weak localization in graphene flakes. Physical Review Letters, 2008. 100(5).

174. Ki, D.-K., et al., Inelastic scattering in a monolayer graphene sheet: A weaklocalization study. Physical Review B, 2008. 78(12).

175. Nikitin, A., et al., $C$-H bond formation at the graphite surface studied with core level spectroscopy. Surface Science, 2008. 602(14): p. 2575-2580.

176. Wang, Z., et al., Synthesis of fluorinated graphene with tunable degree of fluorination. Carbon, 2012. 50(15): p. 5403-5410.

177. Linden, D. and T.B. Reddy, Handbook of batteries. 2002: McGraw-Hill.

178. Read, J., et al., Performance Evaluation of Commercial CFx Materials in Lithium Batteries. 2009, DTIC Document.

179. Tan, T., K. Lam, and H. Yumoto. Li/(CF) n Battery for Low Temperature and High Power Application. in Meeting Abstracts. 2006. The Electrochemical Society. 
180. Chen, K., et al., Hybrid cathode lithium batteries for implantable medical applications. Journal of power sources, 2006. 162(2): p. 837-840.

181. Liu, Y., X. Zhou, and Y. Guo, Effects of reactant dispersion on the structure and electrochemical performance of $L i\langle s u b\rangle 1.2\langle/ s u b\rangle V\langle s u b\rangle 3\langle/ s u b\rangle O\langle s u b\rangle$ $8</ s u b>$. Journal of Power Sources, 2008. 184(1): p. 303-307.

182. Nakajima, T. and N. Watanabe, Graphite fluorides and carbon-fluorine compounds. 1991: CRC Press Boca Raton, FL.

183. Greatbatch, W., et al., Lithium/Carbon Monofluoride (Li/CFx): A New Pacemaker Battery. Pacing and Clinical Electrophysiology, 1996. 19(11): p. 1836-1840.

184. Zhang, S.S., D. Foster, and J. Read, A low temperature electrolyte for primary Li/CF $<$ sub $>x</ s u b>$ batteries. Journal of Power Sources, 2009. 188(2): p. 532-537.

185. Conway, B.E., Electrochemical Supercapacitors: Scientific Fundamentals and Technological Applications. 1999: Springer.

186. Christen, T. and M.W. Carlen, Theory of Ragone plots. Journal of power sources, 2000. 91(2): p. 210-216.

187. Ikeda, H. and S. Narukawa, Behaviour of various cathode materials for non-aqueous lithium cells. Journal of Power Sources, 1983. 9(3): p. 329-334.

188. Ratnakumar, B., et al., Potentiostatic depassivation of lithium-sulfur dioxide batteries on mars exploration rovers. Journal of The Electrochemical Society, 2007. 154(7): p. A715-A724.

189. Klionsky, D.J., et al., Guidelines for the use and interpretation of assays for monitoring autophagy. Autophagy, 2012. 8(4): p. 445-544.

190. Whitacre, J., et al., Low operational temperature $L i-C F<s u b>x</ s u b>$ batteries using cathodes containing sub-fluorinated graphitic materials. Journal of power sources, 2006. 160(1): p. 577-584.

191. Zhang, Z., J. Peng, and H. Zhang, Low-temperature resistance of individual singlewalled carbon nanotubes: A theoretical estimation. Applied Physics Letters, 2001. 79(21): p. 3515-3517.

192. Whitacre, J., et al., Enhanced Low-Temperature Performance of Li-CF x Batteries. Electrochemical and solid-state letters, 2007. 10(7): p. A166-A170.

193. Kozawa, A., Lithium-MnO2 Cells Containing $C F x$ or $C 2 F$ in the Cathode. Journal of The Electrochemical Society, 1987. 134(4): p. 780-782.

194. Gan, H., R.S. Rubino, and E.S. Takeuchi, Dual-chemistry cathode system for highrate pulse applications. Journal of power sources, 2005. 146(1): p. 101-106.

195. Saito, Y., et al., Interlayer spacings in carbon nanotubes. Physical Review B, 1993. 48(3): p. 1907-1909. 


\section{CURRICULUM VITAE}

NAME : Ruwantha Jayasingha Atapattu Mudiyanselage

ADDRESS : Department of Physics and Astronomy

102 Natural Science Building

University of Louisville

Louisville KY 40292

DOB : Colombo, Sri Lanka-Sep 14,1980

EDICATION $\quad$ : B.S., Physics

\&TRANNING University of Kelaniya, Sri Lanka

2002-2006

M.S., Physics

University of Louisville

2008-2010

PhD., Physics

University of Louisville

2010-2013

PROFESSIONAL : Student member of American Physical Society (APS)

SOCIETIES Student member of Kentucky Academy of Science (KAS)

\section{SCHOLARLY PUBLICATIONS}

- Ruwantha Jayasingha, Andriy Sherehiy, Shi-Yu Wu, G. U. Sumanasekera "In-situ study of hydrogenation of Graphene and new phases of localization between metal-insulator transitions ", Submitted to ACS nano (2013)

- Ma, Danhao; Jayasingha, Ruwantha; Hess, Dustin; Adu, Kofi; Sumanasekera, Gamini "Enhancing the Superconducting Temperature of MgB2 by SWCNT Dilution", Submitted to Nano Letters (2012)

- Chakrapani, Vidhya; Abeyweera,Buddika; Jayasingha, Ruwantha; Sumanasekera, Gamini; Angus, John; "Ambient induced changes in the electronic properties of carbon materials: nanotubes, Graphene and carbon fibers", submitted to ACS Nano (2012) 
- Kofi W. Adu, Martin D. Williams, Molly Reber, Ruwantha Jayasingha, Humberto R. Gutierrez, and Gamini U. Sumanasekera, "Probing Phonons in Nonpolar Semiconducting Nanowires with Raman Spectroscopy" Journal of Nanotechnology, vol. 2012, Article ID 264198, 18 pages, 2012.Sidorov, Anton; Gaskill, Kurt; Nardelli, Buongiorno; Tedesco, Joseph; Myers-Ward, Rachel; Eddy, Charles ; Jayasekera, Thushari; Kim, Ki Wook; Jayasingha, Ruwantha: Sherehiy, Andriy; Stallard, Robert; Sumanasekera, Gamini, J. Appl. Phys. 111, 113706 (2012)

- Sidorov, Anton; Sherehiy, Andriy; Jayasingha, Ruwantha; Stallard, Robert; Benjamin, Daniel; Yu, Qingkai; Liu, Zhihong; Wu, Wei; Cao, Helin; Chen, Yong; Jiang, Zhigang; Sumanasekera, Gamini, Appl. Phys. Lett. 99, 013115 (2011)

- Ruwantha Jayasingha, SRD Kalingamudali, Designing of an inexpensive GPIB interfaced voltage source, IEEE Xplore Release 2.4, pp.75-78 (2006)

\section{MEETINGS AND PRESENTATIONS}

- Derek Caplinger, Danhao Ma, Ruwantha Jayasingha, Kofi Adu, Gamini Sumanasekera, "Effect of Carbon Nanotubes on Transport Properties of $\mathrm{MgB}_{2}$ " march meeting of the American Physical Society, Boston, MA, Feb 2012

- Jacek Jasinski, Ruwantha Jayasingha, Kasun Fernando, Christof Keebaugh, Robert Stallard, Gamini Sumanasekera, "Electrical Properties of Hydrogenated Graphene" E-MRS 2011 Fall Meeting, Warsaw University of Technology, Poland, Sep 2011

- Ruwantha Jayasingha, Kasun Fernando, Christof Keebaugh, Robert Stallard and Gamini Sumanasekera, "Thermoelectric Properties of Hydrogenated Graphene" march meeting of the American Physical Society, Dallas, TX, March 2011.

- Andriy Sherehiy, Ruwantha Jayasingha, Robert Stallard, Gamini Sumanasekera, Anton Sidorov, Daniel Benjamin, Zhigang Jiang, Qingkai Yu, Wei Wu, Jiming Bao, Zhihong Liu, Steven Pei, Yong Chen; "Thermoelectric properties of CVD grown large area graphene" march meeting of the American Physical Society, Portland, OR, March 2010

- Ruwantha Jayasingha , Andriy Sherehiy, Gamini Sumanasekera, Anton Sidorov , Zhigang Jiang , Joseph Tedesco , Kurt Gaskill,“ Thermoelectric characterization of large area graphene grown on SiC" 2nd Annual Nanotechnology Symposium, Sullivan University, Louisville, KY, Sep 2010

- Ruwantha Jayasingha, Andriy Sherehiy, Gamini Sumanasekera, Anton Sidorov , Zhigang Jiang , Joseph Tedesco , Kurt Gaskill,“ Thermoelectric

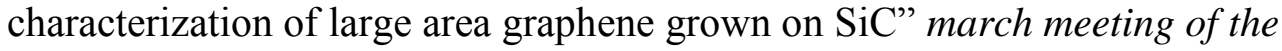
American Physical Society, Portland, OR, March 2010

- Ruwantha Jayasingha, Anton N. Sidorov, Andriy Sherehi, P. J. Ouseph, Gamini Sumanasekera, "Graphene Nanoribbons From Graphite Surface Dislocation Bands" 2nd Annual Nanotechnology Symposium, Sullivan University, Louisville, KY, Oct 2009 


\section{BOOK CHAPTERS}

- Anton N. Sidorov, Ruwantha Jayasingha, Vladimir Dobrokhotov, Gamini Sumanasekera,'In-situ SEM (Scanning Electron Microscope) Deposition and Characterization of Graphene" in Advances in Nanotechnology \& Applications VOL 2, Y. V Pathak and H. T Tran, Eds. Louisville KY: CENTERA, 2010, pp. 173-182.

- Anton N. Sidorov, Ruwantha Jayasingha, P. J. Ouseph, Gamini Sumanasekera, "Monolayer Graphene and its physical properties" in Advances in Nanotechnology \& Applications VOL 1, Y. V Pathak and H. T Tran, Eds. Louisville KY: CENTERA, 2009, pp. 105-126. 